\title{
5
}

\section{Structures and Functions of the Nuclear Envelope}

\author{
Werner W. Franke and Ulrich Scheer
}

I. Introduction $\ldots \ldots \ldots \ldots \ldots \ldots \ldots \ldots \ldots \ldots$

II. The Nuclear Envelope as a Means of Intracellular Compartmentalization ..................... 220

III. Methodological Progress . ............... 223

IV. The Nuclear Envelope as a Part of the Endoplasmic

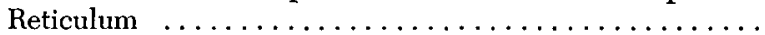

V. Associations of Inner Nuclear Membrane with Chromosomes and Chromatin .................. 244

VI. Association of the Inner Nuclear Membrane with Specific Proteinaceous Layers .................. 256

VII. The Pore Complex ................... 257

A. The Pore Orifice Proper . . . . . . . . 258

B. The Annulus Structures $\ldots \ldots \ldots \ldots \ldots \ldots 259$

C. The Internal Structures of the Pore Complex ... 265

D. Amorphous Material of the Pore Complex ..... 271

E. Pore Complex Models ............... 272

F. Pore Complexes with Dense Equatorial Rings Attached to the Intracisternal Face of the Pore

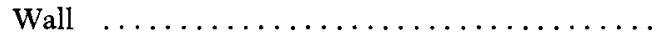

G. Preservation of Nuclear Pore Complex Structures ..................... 274

H. Pore Complexes Compared to Other Membranous Pore Formations ................... 275

I. Pore Complex Formation and Disappearance .. 276

J. Morphological Relationships and Cytochemical Reactions of the Nuclear Pore Complex Constituents $\ldots \ldots \ldots \ldots \ldots \ldots \ldots \ldots \ldots \ldots$

K. Cell Differences in Nuclear Pore Complex Architecture .......................... 284

L. Pore Complex-Connecting Thread Structures . . 286

M. Nuclear Pore Complex Patterns . . . . . . . 288 
N. Number and Frequency of Nuclear Pore

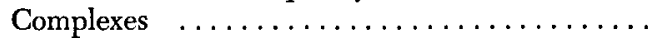

O. Nuclear Pore Complexes and Nucleocytoplasmic Translocation Processes ................. VIII. Relationship of the Nuclear Envelope to Annulate Lamellae (AL) and Intranuclear Cisternae .......... 289 293

IX. Structural Differentiation of the Nuclear Envelope .... 307

$\mathrm{X}$. Associations and Interactions of the Nuclear Envelope with Microtubules and Microfilaments ............

XI. Relationship of the Nuclear Envelope to Other Cell

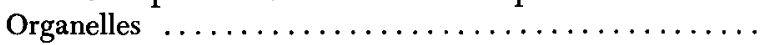

XII. Breakdowns and Reconstitutions of the Nuclear Envelope; Role of the Nuclear Envelope in Nuclear Divisions and

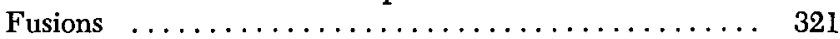

Note Added in Proof $\ldots \ldots \ldots \ldots \ldots \ldots \ldots \ldots \ldots \ldots$

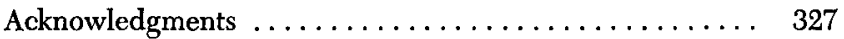

References $\ldots \ldots \ldots \ldots \ldots \ldots \ldots \ldots \ldots \ldots \ldots \ldots . \ldots \ldots$

\section{Introduction}

Although it was first demonstrated indirectly by Hertwig in 1893, the nuclear membrane (or, as it has been called after electron microscopic clarification of its double-membrane architecture, the "nuclear envelope") had been of relatively little interest to both the karyologists and the membranologists. Apart from some isolated early remarks on the molecular orientation of proteins and lipids, derived from studies in polarized light (Chinn, 1938; Schmidt, 1929, 1932, 1937), it was not before the electron microscopic methods and procedures for isolating nuclear membranes had been developed that an essential increase in the knowledge of its organization and biochemistry was noticeable. In the past decade, the structural organization of the nuclear envelope has been the subject of several extensive reviews (e.g., Baud, 1959; Wischnitzer, 1960; Claude, 1964; Gall, 1964; David, 1964; Gouranton, 1969; Stevens and André, 1969; Franke, 1970a; Blackburn, 1971; Feldherr, 1972; Zbarsky, 1972a). It is the aim of this Chapter to summarize the present information on nuclear envelope structures and, further, to discuss their possible functions. A detailed account of the biochemistry of the nuclear envelope is given in Chapter 6.

\section{The Nuclear Envelope as a Means of Intracellular Compartmentalization}

One of the most prominent characteristics of the eukaryotic cell is that its plasma phase is divided into two subcompartments: the cytoplasm 
and the nucleoplasm. The structure that ensures this compartmentalization is the nuclear envelope, a double-membrane system with a somewhat variable cisternal space (the perinuclear cisterna) which frequently shows luminal continuities with the endoplasmic reticulum (ER) channel system (see Fig. 7). Consequently, one finds a polarity of the two cisternal membranes: an outer nuclear membrane, which borders the cytoplasm and is often studded with polyribosomes (like the rough ER) or shows vesicle blebs (like secretory smooth ER), and an inner one, which abuts the nucleoplasm and often is closely associated with chromatin and nuclear ribonucleoprotein structures. In most cells, the perinuclear cisterna is, with variable frequency, interrupted by the pore complexes, i.e., transcisternal orifices comprised of regions of fusion of inner and outer nuclear membrane, which are further conspicuous by a rather uniform diameter and a highly ordered subarchitecture of associated distinct nonmembraneous components. It is obviously a prime function of the nuclear envelope to establish the nucleoplasm as a "zone of exclusion" for ( $a$ ) cytoplasmic organelles such as mitochondria, plastids, and endosymbionts, $(b)$ ribosomes, polyribosomes, "heavy bodies," and various other ribonucleoprotein aggregates, $(c)$ all cisternae of the ER and the dictyosomes, and the various intracellular vesicles, and $(d)$ lipid droplets and polysaccharide storage products including glycogen and the starchlike glucan material in the Rhodophyta. The cytologist can easily distinguish the nucleoplasmic phase as such a zone of exclusion (Fig. 1). This exclusion principle, however, is not perfect in all cells. In special cell types an intranuclear occurrence of free cisternae, some of them even resembling dictyosomes (e.g., Bernhard and Granboulan, 1963; Bucciarelli, 1966; Schultz and Jensen, 1968), of glycogen (e.g., Novikoff, 1957; Binggeli, 1959; Weiss, 1965; Scholz and Paweletz, 1969; Mori et al., 1970; Karasaki, 1971; Paweletz and Granzow, 1972), and of fat droplets (e.g., Thoenes, 1964; Przelecka, 1968; Smyth et al., 1969; Altmann and Pfeifer, 1969; Romen and Bannasch, 1973) has been described. In many cases these intranuclear structures seem to indicate cytopathological changes. An intranuclear occurrence of endosymbionts has also been reported (in Paramecium: Beale et al., 1969; in euglenoid algae: Leedale, 1969).

On the other hand, one cannot consider the nuclear envelope as an absolutely essential structure in the eukaryotic cell as such. An intact nuclear envelope is not principally critical for the viability of an eukaryotic cell. Dramatic nuclear envelope breakdown or delamination from the nuclear chromatin occurs during spermiogenesis in various animals (e.g., Fig. 28; Yasuzumi and Ishida, 1957; Moses and Coleman, 1964; Robison, 1966; Horstmann and Breucker, 1969; Langreth, 1969; Moses and Wilson, 1970; Yasuzumi et al., 1971; Scheer and Franke, 


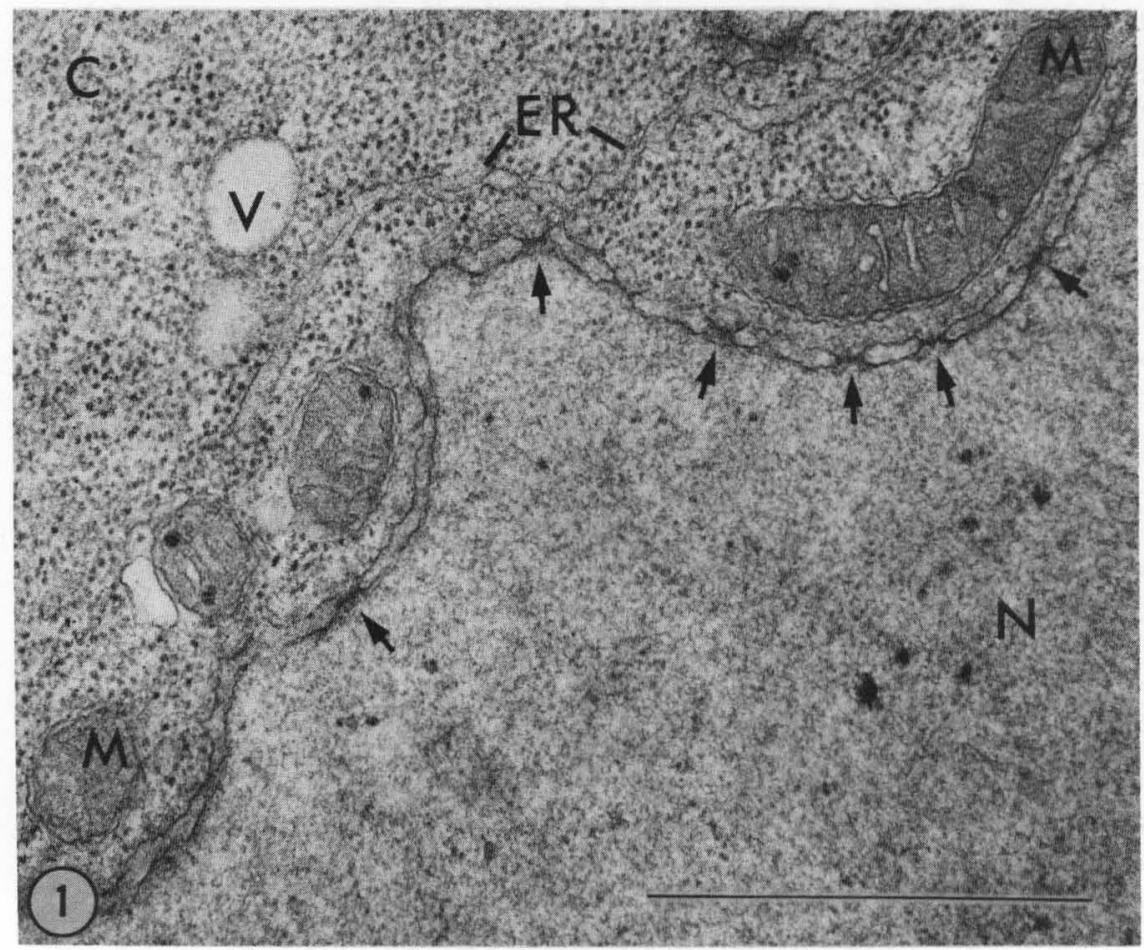

Fig. 1 The nuclear envelope as a compartmentalization barrier between nucleoplasm (N) and cytoplasm (C), demonstrated in a lampbrush stage oocyte of the clawed toad, Xenopus laevis. The perinuclear cisterna which is interrupted at the nuclear pore complexes (arrows) excludes the cytoplasmic components such as the mitochondria $(M)$, the endoplasmic reticulum cisternae (ER), the various vesicles (V) and the ribosomes from the nuclear interior. Note that similar small "zones of exclusion" are also recognized between the nuclear envelope and the outer membrane of the juxtanuclear mitochondria $(\times 45,000$, bar indicates $1 \mu \mathrm{m})$.

1974) and perhaps also plants (Diers, 1967), and in some mature sperm, for instance, in coccid insects, the chromatin is totally naked (e.g., Moses and Wilson, 1970). In other sperm cells the nuclear envelope breaks into fragments, or disappears totally, after penetration into the ooplasm and does not reconstitute until formation of the male pronucleus (e.g. Longo and Anderson, 1968; Stefanini et al., 1969; Zamboni, 1971). Moreover, some types of mitotic and meiotic nuclear divisions are characterized by a transient disintegration of the perinuclear cisterna (e.g., between prometaphase and late anaphase in the "open" or "polar fenestrae" mitoses; see Section XII). These two examples of nuclear envelope disintegration refer to cell stages known to have decreased rates of RNA 
and protein synthesis, but the literature also contains reports of extensive nuclear envelope disintegrations in cellular states of high activity in RNA and protein synthesis, namely, the silk gland cells of Bombyx mori (Tashiro et al., 1968) and the rostellar gland cells in a cestode, Echinococcus granulosus (Smyth et al., 1969). It would be of great importance to ascertain that such nuclear envelope "gaps" are not artifactual (compare also King and Devine, 1958; Okada and Waddington, 1959).

A very special case of compartmentalization of the ground cytoplasm occurs in some bryopsidacean and dasycladacean green algae such as Acetabularia and Bryopsis. In these relatively large cells a giant nucleus lies in the rhizoidal extensions and is surrounded not only by a "true" nuclear envelope, but also by another porous, more inflated cisterna which is continuous with vacuolar spaces of the cytoplasm (Fig. 2; Werz, 1964; Boloukhère, 1970; Burr and West, 1971; Franke et al., 1974). This perinuclear lacuna excludes cytoplasmic organelles and particles not only from the nucleoplasm but also from a special intermediate compartment, the perinuclear zone, situated between the true and the "secondary" nuclear envelope (Fig. $2 b$ and c). This formation of an accessory perinuclear envelope is typical for the giant primary nucleus and is lost during the formation of the smaller "secondary nuclei" which then migrate upward in the cell stalk and finally become cyst nuclei (Boloukhère, 1970; Woodcock, 1971; Woodcock and Miller 1973).

\section{Methodological Progress}

Recent progress in studies on the chemistry and structure of the nuclear envelope has come mainly from isolation techniques developed in several laboratories. The methods for isolating nuclear envelope material can be classified into two main groups. (a) Manual preparations of individual nuclear envelopes have been performed primarily with giant nuclei, in particular with oocytes from amphibia and echinoderms, and with the primary nuclei of dasycladacean algae. Such techniques are essentially based upon the early work of Callan and Tomlin (1950): the nuclear envelope is separated with fine forceps under a binocular as a "ghost" from the isolated nucleus (Fig. 3), and is then washed by repeated sucking up and down in a pipette. For several years this kind of preparation had been restricted to structural studies (Gall, 1954, 1956, 1959, 1964, 1967; Merriam 1961, 1962), but has been recently scaled up to be useful for biochemical determinations (Scheer, 1972), although the maximum production rate is only ca. 200 nuclear envelopes per day per worker. In oocytes, the particular advantages of this method 


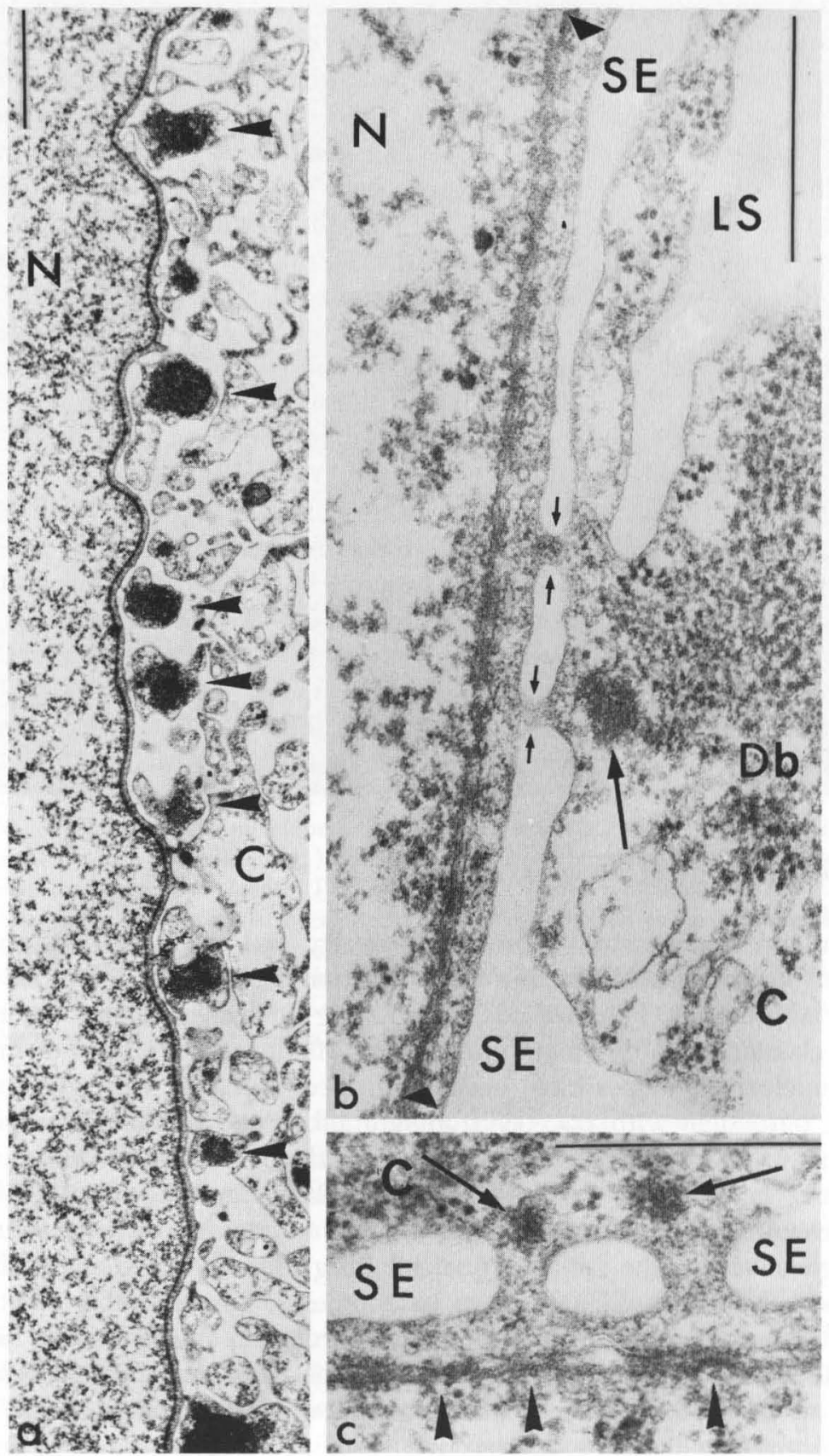


are $(i)$ the easily controllable high purity, especially with respect to cytoplasmic contaminants (Fig. $3 \mathrm{~b}$; for quantitative data see Scheer, 1972); (ii) that one can prepare, in the presence of sufficient divalent cations, the nucleoplasm and the nuclear envelope from the same nucleus (Fig. 3a), thus having in hand an optimal recovery system; (iii) the good preservation of the large envelope pieces (Figs. 3 and 4); and (iv) that one deals with defined single cells and with a determinable amount of membrane area and pore complexes. With the giant nuclei of green algae, however, the nuclear envelope is in most cases seriously contaminated by the adhering perinuclear lacuna and the layer of finely filamentous material sandwiched between. $(b)$ Mass isolations of nuclear membrane material usually start from a purified fraction of isolated nuclei. The nuclei are then fragmented and/or extracted with combinations of diverse treatments: (i) rapid chromatin swelling in hypotonic solutions (Franke 1966a, b, 1967a, b; Zbarsky et al., 1967, 1969; Franke and Kartenbeck, 1969; Price et al., 1972; reviewed by Zbarsky, 1972a, b); (ii) vigorous homogenization, shearing and sonication (Franke, 1966a, b, 1967a, b; Bornens, 1968; Kashnig and Kasper, 1969; Zbarsky et al., 1969; Comes and Franke, 1970; Franke et al., 1970a; Harris and Agutter, 1970; Harris and Brown, 1971; Zentgraf et al., 1971; Agutter, 1972; Fakan et al., 1972; Moore and Wilson, 1972; Jarasch et al., 1973); (iii) limited digestion of the chromatin with deoxyribonuclease (Berezney et al., 1970, 1972; Kay et al., 1971, 1972; Zentgraf et al., 1971; Matsuura and Ueda, 1972); (iv) destabilization with chelating agents such as citrate (Bornens, 1968; Kashnig and Kasper, 1969) or in high salt concentrations (Berezney et al., 1970, 1972; Franke et al., 1970; Zent-

Fig. 2 Special nucleocytoplasmic separation zone of the primary (giant) nucleus in the rhizoid of the green alga, Acetabularia mediterranea. Here the cytoplasm (C) does not border on the nuclear envelope ( $N$, nucleus) but to a special cisterna which constitutes a "secondary envelope" ( $\mathrm{SE}, \mathrm{b}$ and c). In the approximately $700 \AA$ broad zone between this secondary envelope and the nuclear envelope (pore complexes are denoted in $c$ by arrowheads) which is marked by the triangles in $b$, one recognizes only finely fibrillar structures and small vesicular and tubular profiles (diameter ca. $300 \AA$, see the central part in b). This "perinuclear lacuna" is also perforated by cisternal pores (denoted in b by the small arrows) which, however, are different from nuclear pore complexes. Note the regular distribution of large, densely stained aggregates in the juxtanuclear cytoplasm (denoted by arrowheads in a) which often reveal two subcomponents, a dense body (Db) constituted by ca. $250 \AA$ large granular particles and a very dense smaller aggregate (up to $0.15 \mu \mathrm{m}$ in diameter, large arrows in $b$ and $c$ ) which lies on the cytoplasmic side of the secondary envelope pores. LS, lacuna spaces in the rhizoidal cytoplasm ( $a, \times 7000$, bar indicates $2 \mu \mathrm{m} ; \mathrm{b}, \times 58,000 ; \mathrm{c}, \times 64,000$, bars indicate $0.5 \mu \mathrm{m})$. 


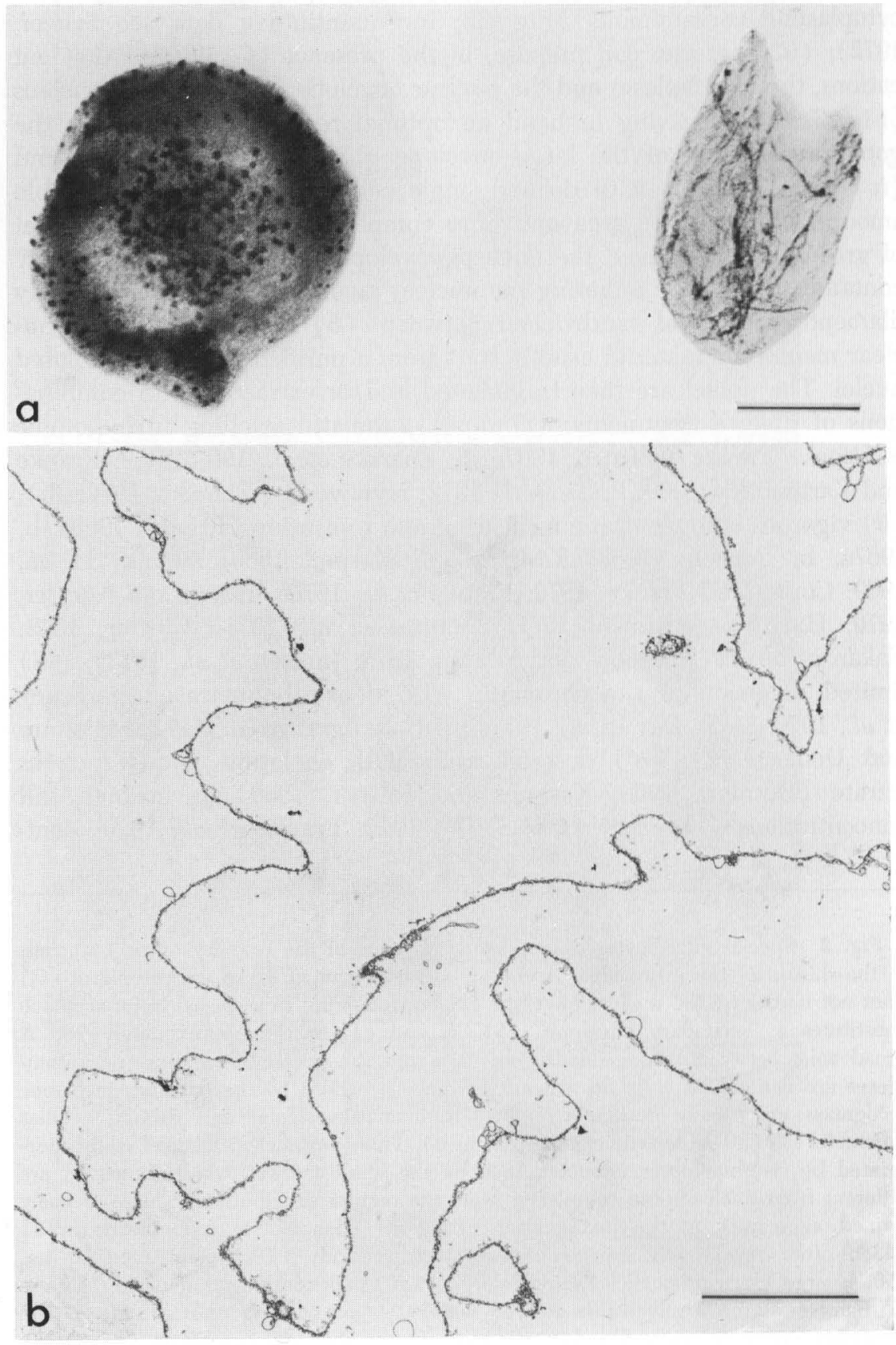

(3) 
graf et al., 1971; Matsuura and Ueda, 1972; Monneron et al., 1972; Moore and Wilson, 1972).

In most preparative methods, the nuclear membrane fragments are then separated from nonmembraneous nuclear components by making use of the clearly lower buoyant density of membrane lipoproteins by sedimentation or flotation in concentration gradients of sucrose (continuous or discontinuous), sorbitol, or CsCl (Franke, 1966, b, 1967a, b; Kashnig and Kasper, 1969; Zbarsky et al., 1969; Franke et al., 1970; Kay et al., 1971, 1972; Zentgraf et al., 1971; Agutter, 1972; Berezney et al., 1972; Fakan et al., 1972; Matsuura and Ueda, 1972; Monneron et al., 1972; Moore and Wilson, 1972; Price et al., 1972). Methods for preparing nuclear membrane fractions have been reported for various plant tissues such as onion root tip or leaves (Franke, 1966b), for the macronuclei of the ciliate, Tetrahymena pyriformis (Franke, 1967a, b; Eckert, 1972), for mouse, rat, rabbit, and pig liver (Franke, 1967a, b; Zbarsky et al., 1967, 1969; Bornens, 1968; Kashnig and Kasper, 1969; Berezney et al., 1970, 1972; Franke et al., 1970; Kartenbeck et al., 1971, 1973; Agutter, 1972; Kay et al., 1972; Monneron et al., 1972; Price et al., 1972), for avian erythrocytes (Harris and Brown, 1971; Zentgraf et al., 1971) for rat and calf thymus (Matsuura and Ueda, 1972; Jarasch et al., 1973), for rat prostate gland (Moore and Wilson, 1972), and for a series of tumor cells (Zbarsky et al., 1967, 1969; Comes and Franke, 1970; Fakan et al., 1972; Green and Dobrjansky, 1972; Price et al., 1972). The variety of procedures reported has been reviewed in detail by Zbarsky (1972b) and Franke (1974).

The purity and structural preservation of the isolated nuclear membranes can be examined in the electron microscope in both thin section (Franke, 1966b; Bornens, 1968; Kashnig and Kasper, 1969; Zbarsky et al., 1969; Kay et al., 1972; Monneron et al., 1972; Price et al., 1972; Scheer, 1972; Kartenbeck et al., 1973; see also Chapter 6 of this volume) and in negatively stained preparations, the latter preferably done with neutral solutions of tungstate salts (Figs. 5 and 6; Gall, 1964, 1967; Franke, 1966b, 1967b; Franke and Kartenbeck, 1969; Berezney et al., 1970, 1972; Comes and Franke, 1970; Franke and Scheer, 1970a; Franke

Fig. 3 The nuclear envelope and the (aggregated) nuclear contents can be separated by hand from an isolated giant nucleus of an amphibian cocyte. (a) A light micrograph which shows, in Triturus alpestris, both products separated: the nuclear contents (with numerous nucleoli) in the left and the whole nuclear envelope "ghost" in the right. (b) A survey electron micrograph which demonstrates the purity and structural integrity of such an isolated nuclear envelope (in this case from a Xenopus laevis oocyte) (a, $\times 80$, bar indicates $200 \mu \mathrm{m} ; \mathrm{b}, \times 4000$, bar indicates $1 \mu \mathrm{m}$ ). 


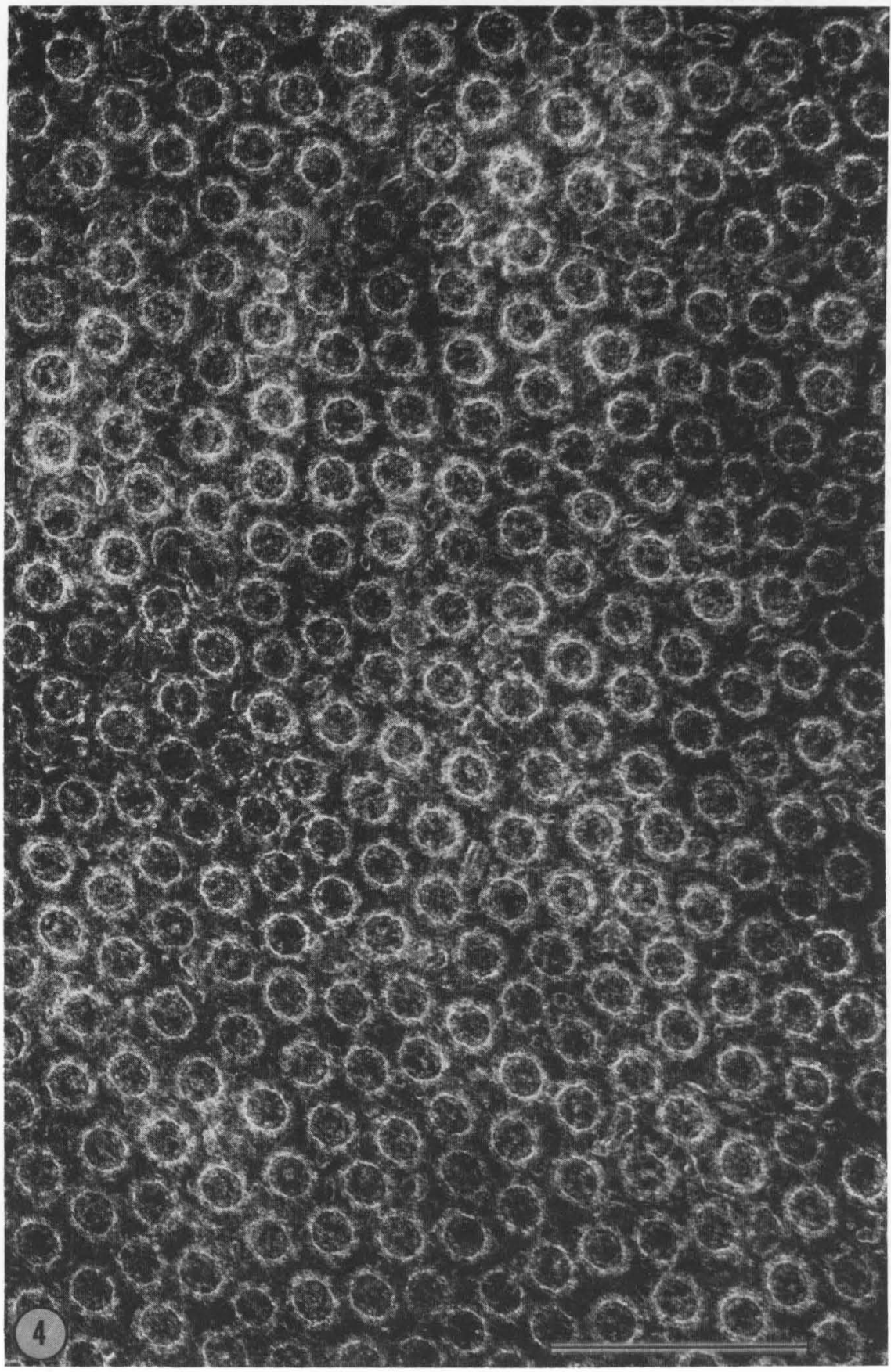


et al., 1970; Kartenbeck et al., 1971; Kay et al., 1972; Scheer, 1972; Fabergé, 1973). The pore complexes provide a suitable structural marker for positive identification of nuclear membrane fragments, provided that the fragments are large enough. The size of envelope fragments appears to depend greatly on the mechanical stress exerted during preparation and on cell type-specific differences in nuclear membrane fragility. In preparations in which the nuclei have been ruptured by swelling or gentle homogenization, the pieces are larger than after extensive sonication (compare Figs. 5 and 6). In our and other authors' experience the kind of ion used for the high ionic strength extraction does not specifically affect the fragment size (e.g., Franke et al., 1970; Deumling, 1972; Monneron et al., 1972; Kartenbeck et al., 1973; however: Agutter, 1972). Likewise, the relative amounts of cisternal envelope fragments, small vesicular formations, and inner and outer lamellar membrane sheets are strongly variable depending on the specific preparation method. A delicate, and so far not finally solved, problem concerns the quantitative determination of contaminants, especially from the nucleoplasm and, as a consequence of the morphological membrane continuity, from the ER. While the presence of nucleus-interior markers (e.g., pre-rRNA, specific DNA and RNA polymerases, NAD-pyrophosphorylase) can be assayed, the amount of microsomal contamination is hard to determine since an absolute marker substance discriminating between nuclear and microsomal membrane is not yet known (for controversial statements concerning the presence of NADH-cytochrome C-reductase and glucose-6-phosphatase in mammalian liver nuclear membranes, see Kashnig and Kasper, 1969; Zbarsky et al., 1969; Berezney et al., 1970, 1972; Franke et al., 1970; Kasper, 1971; Kartenbeck et al., 1973; for details see Chapter 6). Membranolytic detergents have also been used in attempts to prepare nuclear membrane material, specifically for enrichment of inner nuclear membrane (Bach and Johnson, 1966; Whittle et al., 1968; Ueda et al., 1969; Ben-Porat and Kaplan, 1971). These seem to be of very limited value, since they induce varying amounts of structural damage (Kartenbeck et al., 1973). Yoo and Bayley (1967) isolated nuclear envelope fragments from pea roots after a pre-

Fig. 4 Electron micrograph of a nuclear envelope prepared from a Xenopus laevis oocyte (lampbrush stage) as described in the previous figure which displays, in negative staining with neutral sodium phosphotungstate solution, the abundance and dense package of the nuclear pore complexes. In this particular preparation the conditions (compare Franke and Scheer, 1970a) were such that the annular and internal material of the pore complex was partially removed, in order to demonstrate the membrane rims of the pores more clearly $(\times 66,000$, bar indicates $0.5 \mu \mathrm{m})$. 


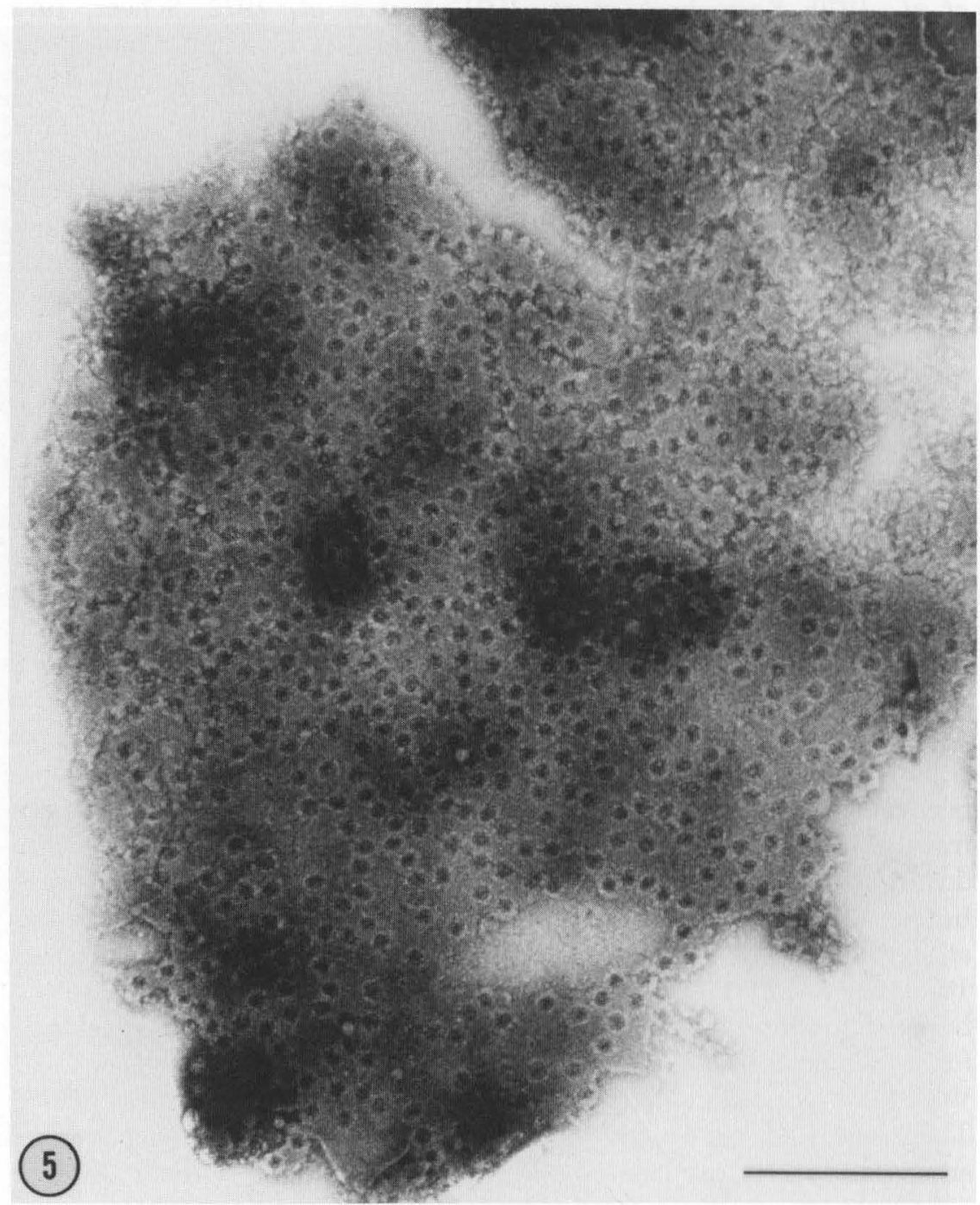

Fig. 5 Nuclear envelope mass preparation from isolated onion root tip nuclei, showing large envelope fragments as they are typically obtained after hypotonic swelling shock and limited sonication; negative staining as in Fig. 4 ( $\times 24,000$, bar indicates $1 \mu \mathrm{m}$ ).

stabilization of the tissue by glutaraldehyde fixation, a method which is suitable for structural investigations, but precludes most biochemical experiments. 


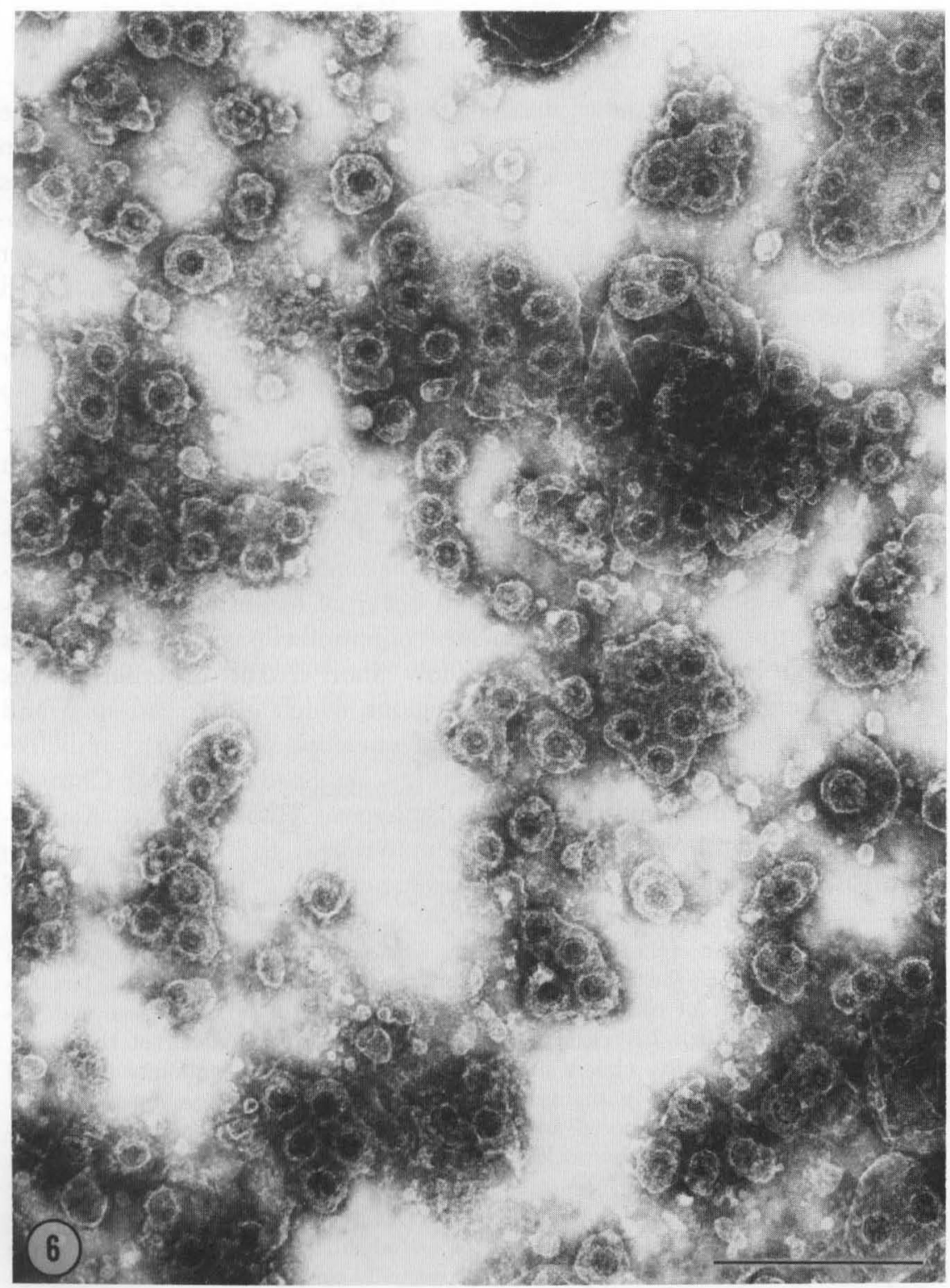

Fig. 6 Negatively stained (Na-phosphotungstate, $\mathrm{pH}$ 7.2) fraction of small nuclear envelope fragments isolated from rat liver (for details of preparation see Franke, 1967b, and Kartenbeck et al., 1971) as they are produced by extensive sonication. The purity of the fraction is indicated by the existence of pores in nearly all the membraneous fragments $(\times 50,000$, bar indicates $0.5 \mu \mathrm{m})$. 


\section{The Nuclear Envelope as a Part of the Endoplasmic Reticulum}

In most cells, the outer nuclear membrane is continuous with the membranes of the ER at many sites (Fig. 7; Watson, 1955). Only in cells that lack considerable amounts of ER (e.g., mature erythrocytes from birds or amphibia; late stages in spermiogenesis) are such continuities not detected. Particularly frequent is the aspect that the outer nuclear membrane is studded with polyribosomes, as are the rough ER cisternae with which it is continuous (Fig. 7a and b). In some cell types, juxtanuclear cisternae of rough ER are distinct from cisternae in other parts of the cytoplasm by showing regular ergastoplasmlike stacking, by constituting special morphological formations (as, for instance, the amplexus present in various algae and fungi; Lang, 1963; reviews: Brown and Bertke, 1969; Morré et al., 1971a; Whaley et al., 1971), or by the appearance of more or less frequent pore complexes, which give rise to the perinuclear annulate lamellae (AL) formations (Fig. 7d; reviews: Kessel, 1968a; Wischnitzer, 1970). Not all cisternal extensions from the nuclear envelope into the cytoplasm are communications with other parts of the whole ER system. Some cells show "short circuit" cisternal bridges between neighbor nuclear envelope regions which either can span and connect the invaginations of the nuclear envelope or are just handlelike outgrowths of the nuclear envelope (Figs. 7c, 8a-d). In most Chromophyta algae (in particular in the Chrysophyceae, Haptophyceae, Xanthophyceae, Bacillariophyceae, and Phaeophyceae), the nuclear envelope constitutes, where the chloroplast lies against the nucleus, a part of

Fig. 7 Transverse sections demonstrating continuities of the perinuclear cisterna with the cisternae of the endoplasmic reticulum (ER, a-c) and annulate lamellae ( $A L, d$ ). (a) A site of continuity of the (rough) outer nuclear membrane with the membranes of a rough ER cisterna in a meristematic root tip cell of the onion, Allium cepa, fixed in situ. (b) A similar situation (the site of continuity is denoted by the two small arrows in the very right) in a lampbrush stage Xenopus laevis oocyte fixed in situ. The curved arrows denote ribosomal arrays (perhaps polyribosomes) extending between the nuclear envelope and the adjacent rough ER cisterna, thus apparently connecting both membrane surfaces. (c) In an isolated nuclear envelope from a mature Xenopus laevis oocyte, a luminal continuity of the perinuclear space with a spongelike network of ribosome-studded cisternae and tubules, which is equivalent in character to rough ER but apparently constitutes an isolate membrane extension of the nuclear envelope. (d) Typical appearance of a juxtanuclear AL stack (note the many pore complexes in the annulate cisternae) which is in manifold luminal continuity with the perinuclear cisterna via short cisternal bridges. The arrowheads in $a$ and $b$ point to nuclear pore complexes. $N$, nuclear side; $C$, cytoplasmic side (a, $\times 54,000 ; \mathrm{b}, \times 37,000 ; \mathrm{c}, \times 47,000$, bars indicate $0.5 \mu \mathrm{m}$; $\mathrm{d}$, $\times 25,000$, bar indicates $1 \mu \mathrm{m})$. 


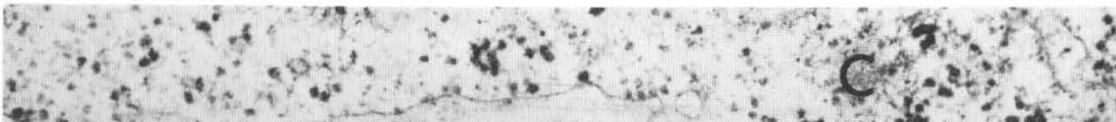
ER $\quad$ on

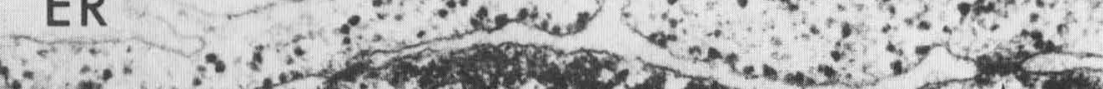

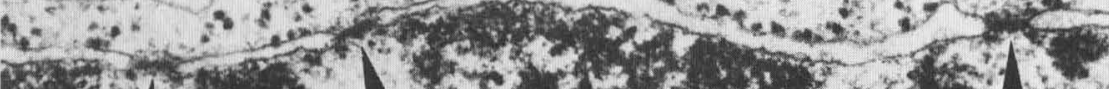

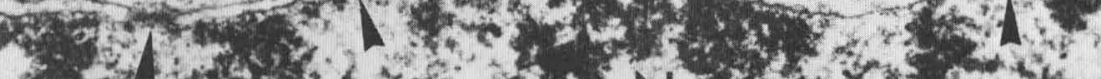

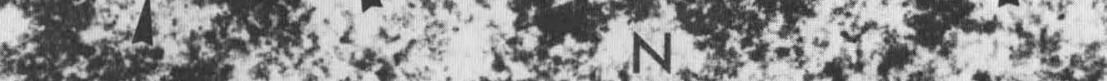

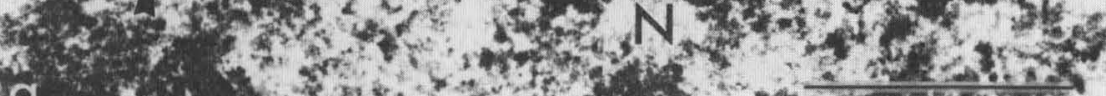

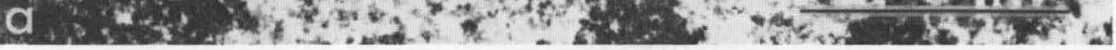

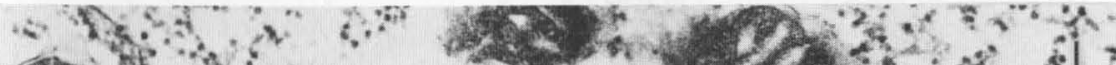

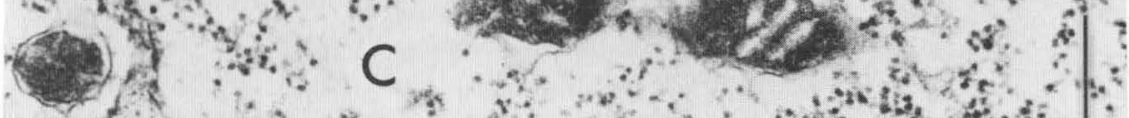

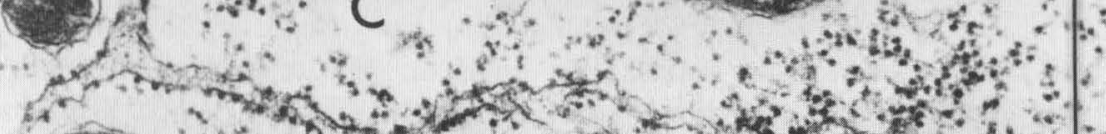

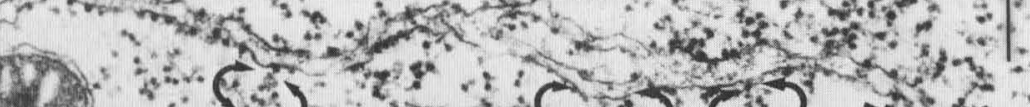

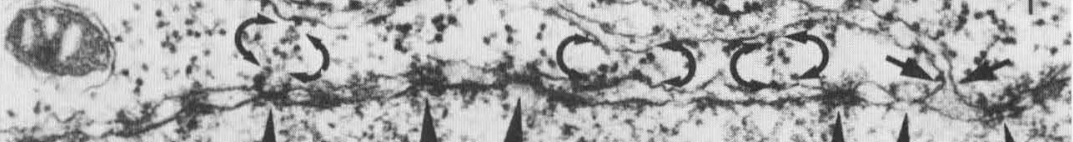
$\therefore$ -

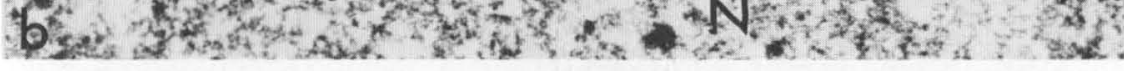
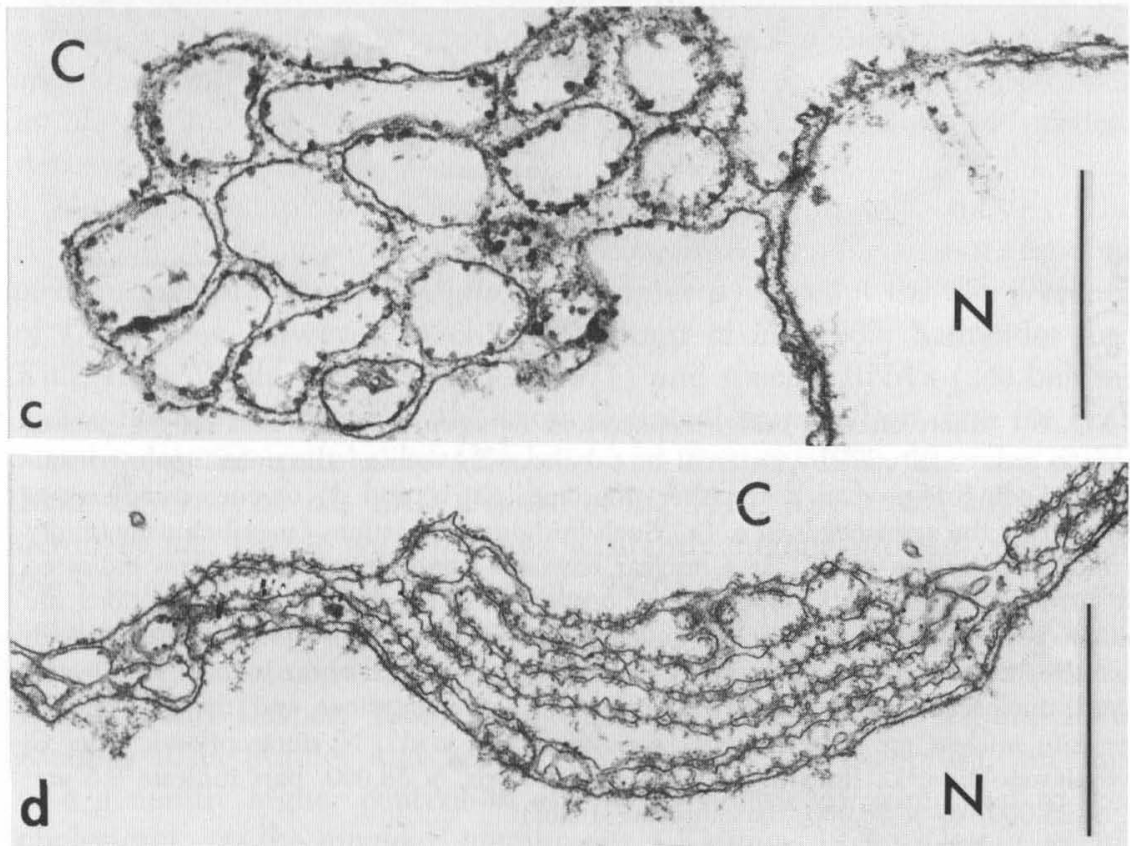

(7) 

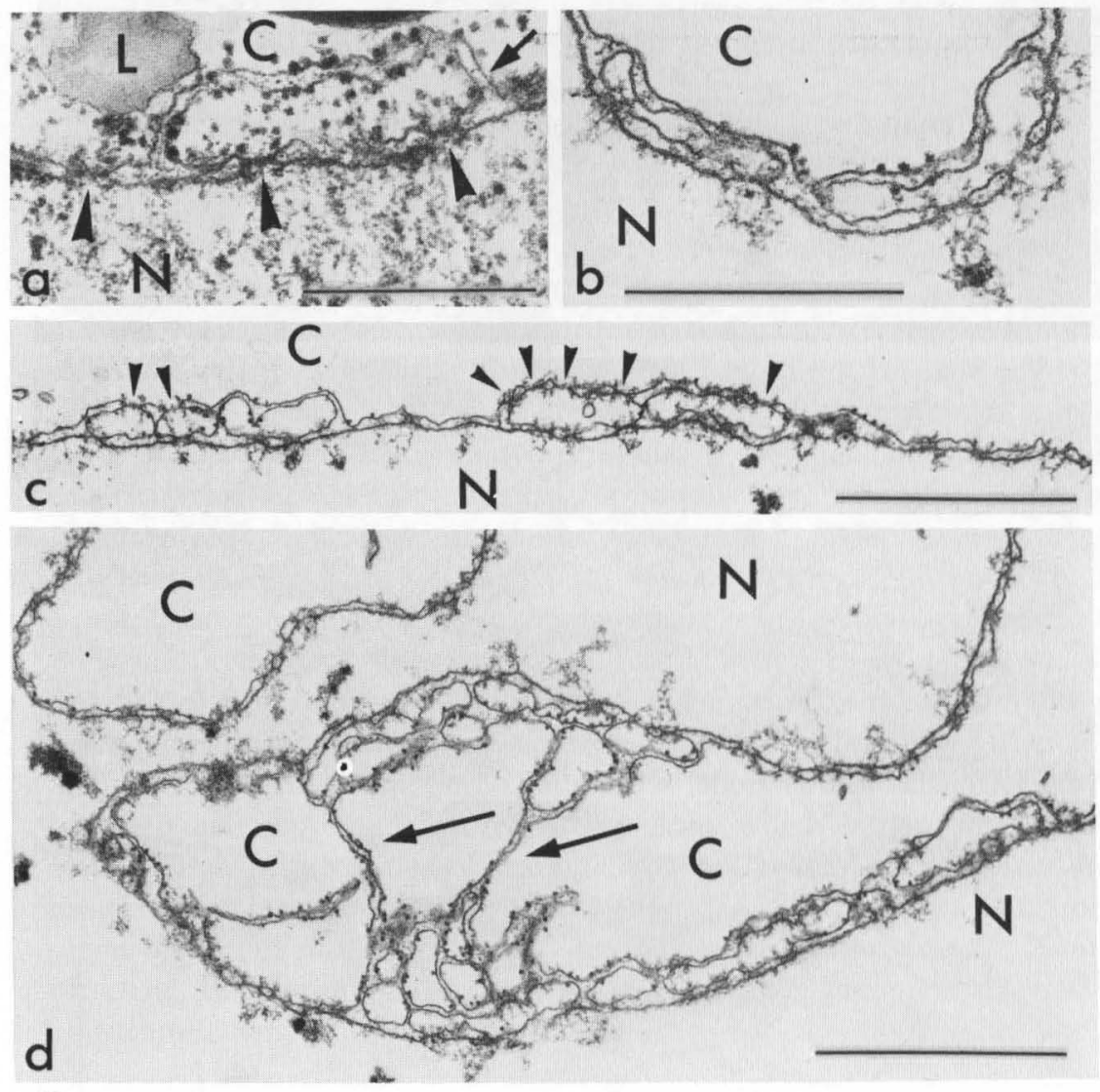

(8)

Fig. 8 "Short circuit" bridge connections between neighbor sites on the nuclear surface are constituted by cisternal or tubular extensions of the outer nuclear membrane. Such bridges can bear either ribosomes ( $a, b$, and d) or pore complexes as denoted by the arrowheads in c. (a) Such bridging connections (membrane continuity is indicated by the arrow) at a nuclear envelope (nuclear pore complexes indicated by arrowheads) in a Xenopus laevis oocyte fixed in situ. These connections are retained with the isolated nuclear envelopes (b-d). They can show smooth or rough character as well as pore complexes in adjacent regions ( $b$ and $c$ ). In highly invaginated nuclear envelopes such bridges span the indentations and thereby connect opposite nuclear envelope regions (e.g., at arrows in d). N, nucleoplasmic side; C, cytoplasmic side; L, lipid droplet ( $\mathrm{a}, \times 48,000$; b, $\times 58,000$, bars indicate $0.5 \mu \mathrm{m}$; c, $\times 25,000 ; \mathrm{d}, \times 26,000$, bars indicate $1 \mu \mathrm{m})$. 
the chloroplast ER ("periplastidal cisterna") or is connected to it by short cisternal bridges (Fig. 9; Gibbs, 1962, 1970; Bouck, 1965; Falk, 1967; Falk and Kleinig, 1968). This association secures in these organisms a stable linkage of the chloroplast( $s)$ to the nucleus.

Structurally, the nuclear membranes belong, in the endomembrane system, to the "ER-type" membranes (for definition see Morré et al., 1971a), corresponding to the $\alpha$ - and $\gamma$-cytomembranes in the terminology of Sjöstrand (1968). They are usually thinner (50-85 \& thickness in sections, $75-110 \AA$ in freeze-etch preparations; compare Kartenbeck et al., 1971) and, in many cells, show less frequently and clearly the dark-light-dark (unit membrane) pattern than, as the extreme counterpart, the plasma membrane (Yamamoto, 1962, 1963; Grove et al., 1968; Morré et al., 1971a). In a given cell system they represent an early stage in the membrane differentiation (maturation) sequence of membrane structural differences (Grove et al., 1968; Keenan and Morré, 1970; Morré et al., 1971a). Except for some specializations in membrane structure (see below), and the fact that the inner nuclear membrane interacts with structures of the nuclear interior, the nuclear membranes are not distinguishable from other regions of the ER. In freeze-cleaveetch preparations, nuclear membranes exhibit the same pattern of intramembrane fracturing as other membranes (Branton, 1966; Branton and Deamer, 1972; Monneron et al., 1972; for review see Kartenbeck et al., 1971), even with respect to specific configurations in particle decoration (Breathnach et al., 1972).

The relationship of the nuclear envelope membranes to those of the rough ER is also apparent from the biochemical comparisons of fractions of both membranes (for details see Chapter 6). Except for the presence of DNA (see, however, Bach, 1962; Bond et al., 1969; Schneider and Kuff, 1969; Williamson, 1970; Bell, 1971) and specific RNA's (see below) in the nuclear membranes, the gross chemical composition data for both membranes are quite alike (Kashnig and Kasper, 1969; Zbarsky et al., 1969; Berezney et al., 1970; Franke et al., 1970; Kay et al., 1972; Monneron et al., 1972), with perhaps a slightly higher protein content in the nuclear envelope (Franke et al., 1970). Rough ER and nuclear envelope membranes have almost identical total lipid, phospholipid, and fatty acid patterns (Gurr et al., 1963; Lemarchal and Bornens, 1969; Kashnig and Kasper, 1969; Franke et al., 1970; Keenan et al., 1970; Kleinig, 1970; Stadler and Kleinig, 1971), with the exception, perhaps, of a generally higher content in esterified cholesterol, compared to free cholesterol, in the nuclear membranes (Kleinig, 1970; Kleinig et al., 1971; Sato et al., 1972). Both membranes differ, however, from dictyosome and plasma membranes in the same cell in their lipid composition 


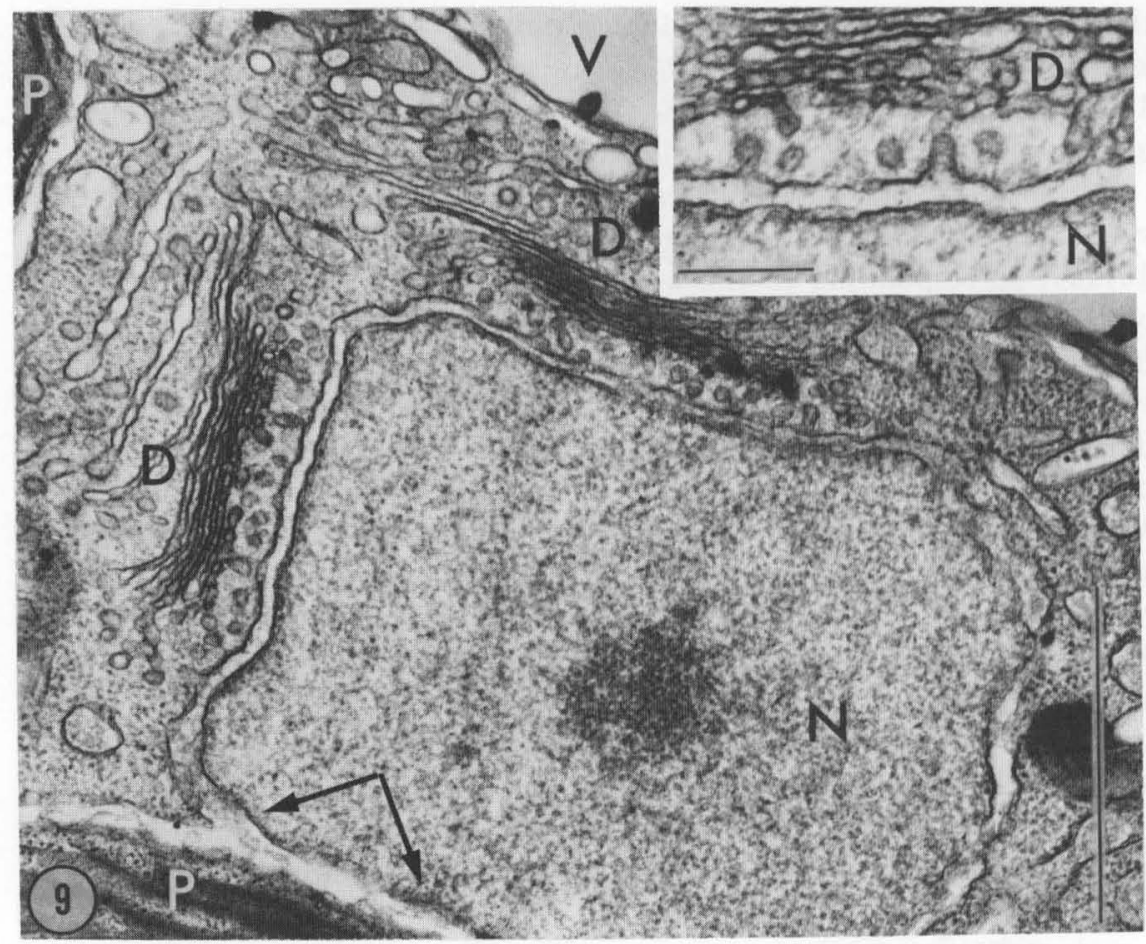

Fig. 9 Relationship of the nuclear envelope and the Golgi apparatus in the Xanthophycean alga, Botrydium granulatum. The two dictyosomes (D) constituting one Golgi apparatus are oriented with their forming (proximal) faces toward distinct, smooth-surfaced regions of the nuclear envelope which are characterized by a high blebbing activity at the outer nuclear membrane (inset). Note also the continuity of the perinuclear space with the periplastidal cisterna (pair of arrows in the lower left). N, nucleus; $\mathrm{P}$, plastid; $\mathrm{V}$, vacuole $(\times 34,500$, bar indicates $1 \mu \mathrm{m}$; inset, $\times 68,000$, bar indicates $0.2 \mu \mathrm{m}$ ). (Electron micrograph courtesy of Dr. H. Falk.)

(see previous references and Glaumann et al., 1968; Pfleger et al., 1968; Ray et al., 1969; Keenan and Mooré, 1970; Konings and Loomeijer, 1970; Kleinig et al., 1971; Zentgraf et al., 1971; Sato et al., 1972; Jarasch et al., 1973). Furthermore, gel electrophoretic protein patterns of both membrane fractions show marked homologies, though some bands are specific for the one or the other (Franke et al., 1970; Deumling, 1972; Monneron et al., 1972; for detailed discussion see Chapter 6). The same cytochrome pigments have been found in both fractions, although their relative content was found to be somewhat lower, on a protein weight basis, in the nuclear membranes (Ueda et al., 1969; Franke et al., 1970; 
Berezney and Crane, 1971, 1972; Fleischer et al., 1971; Kasper, 1971; Berezney et al., 1972; Ichikawa and Mason, 1973). Patterns of nuclear membrane-bound enzyme activities are also generally identical with those in rough microsomes (a vesicle fraction derived from the rough ER), although quantitative differences per protein mass have been reported: for instance, in mammalian liver the microsomal marker enzyme activities glucose-6-phosphatase and NADH- and NADPH-cytochrome $\mathrm{C}$ reductases have been reported to be lower in the nuclear membranes (Zbarsky et al., 1968, 1969; Kashnig and Kasper, 1969; Berezney et al., 1970, 1972; Franke et al., 1970; Kasper, 1971; Zentgraf et al., 1971; Ichikawa and Mason, 1973; Green and Dobrjansky, 1972; Kay et al., 1972; Kartenbeck et al., 1973; Franke, 1974; for cytochemical references see further Goldfischer et al., 1964; Leskes and Siekevitz, 1969; Kartenbeck et al., 1973). On the other hand, a relative enrichment of NADPH$\Delta^{4}-3$-ketosteroid-5-reductase has been described in rat prostate gland nuclear membranes by Moore and Wilson (1972). The presence of cyclic $3^{\prime}, 5^{\prime}$-adenosinemonophosphatase in nuclear membranes has recently been suggested for human lymphocytes by Coulson and Kennedy (1972).

While $\mathrm{Mg}^{2+}$-stimulated adenosinetriphosphatase (ATPase) activity is apparently associated with nuclear envelopes, the monovalent cationstimulated ATPase seems to be absent (Delektorskaya and Perevoshchikova, 1969; Kashnig and Kasper, 1969; Zbarsky et al., 1969; Franke et al., 1970a; Zentgraf et al., 1971; Jarasch et al., 1973). This is in contrast to its occurrence in the microsomal fractions. As opposed to ER membranes, cytochrome oxidase and oxidative phosphorylation activities have been reported in nuclear and nuclear membrane fractions from mammalian liver and thymus (Zbarsky et al., 1968, 1969; Kuzmina et al., 1969; Berezney and Crane, 1971, 1972; Berezney et al., 1972; Matsuura and Ueda, 1972; Franke, 1974; Jarasch and Franke, 1974; see also Chapter 6). In our opinion, however, these findings may be attributed to contamination by mitochondrial membrane material rather than to their endogeneous presence in nuclear membranes in vivo. Likewise, the occurrence of cardiolipin in nuclear membrane fractions (Keenan et al., 1970, 1972; see, however, Kleinig, 1970; Kleinig et al., 1971; Sato et al., 1972; Jarasch et al., 1973) might be due to such contamination.

The in vivo incorporation kinetics of lipid precursors and amino acids into the membrane lipoproteins exhibit similar kinetics in both, the rough ER and the nuclear envelope, and their in vitro capacity for incorporating amino acids and CoA-activated or nonactivated fatty acids is similar (Hallinan et al., 1966; Widnell and Siekevitz, 1967; Franke et al., 1971a; Morré et al., 1971a; Deumling, 1972; Deumling and Franke, 1974; Stadler and Franke, 1973). An enrichment in the nuclear membranes, over its 
presence in microsomes, has been noted for the in vitro binding of the antimitotic alkaloid, colchicine (Stadler and Franke, 1972, 1974).

As is the case with rough ER polyribosomes, it is widely assumed that the nuclear envelope (or to be specific, its outer membrane) plays a role in the synthesis of two different kinds of proteins, membranebound polypeptide chains and secretory proteins. That a single defined protein can be deposited in the perinuclear cisterna as well as in other spaces of the rough ER has been most elegantly shown for the synthesis of ferritin and peroxidase antibodies in plasma cells (De Petris et al., 1963; Avrameas and Bouteille, 1968; Leduc et al., 1968, 1969; Avrameas, 1970). Evidence was also presented in these reportes that synthesis of the antibody protein occurs at the nuclear envelope before it spreads over the rough ER cisternae more distant from the nucleus. Incorporation of amino acids into both membraneous and intracisternal proteins of the nuclear envelope has also been found in vitro using isolated nuclei (Deumling and Franke, 1974). It is not known whether the polyribosomes on the outer nuclear membrane synthesize proteins of the cytoplasmic phase ("cytosol") and/or of the nucleoplasm, and whether they preferentially or exclusively read some specific messages, like those coding for some histones, as suggested by Gorovsky (1969).

The occurrence of some glycoproteins rich in mannose and glucosamine in isolated rat liver nuclear membranes was recently reported (Kawasaki and Yamashina, 1972; compare also Kashnig and Kasper, 1969). The data of these authors suggest that the glycopeptide pattern of the nuclear membranes is identical in inner and outer membrane but differs from that of other endomembranes and plasma membrane by the relatively low level of sialic acid; and further, that these compounds are synthesized in loco rather than being transported from other membranes.

That not only the production but also the (perhaps transitory) storage of intracisternal proteins, secretory proteins included, occurs in the nuclear envelope has also been indicated in cytochemical studies of the distribution of peroxidase (Poux, 1969; Fahimi, 1970; Herzog and Miller, 1970, 1972; Strum and Karnovsky, 1970; Strum et al., 1971) and by the many observations of structured proteins within the perinuclear space. Examples of this latter are not only the conspicuous intracisternal protein crystals which sometimes even locally expand the nuclear membranes (Behnke and Moe, 1964; Marquet and Sobel, 1969; Perrin, 1969, 1970; for reviews see: Wergin et al., 1970; Blackburn, 1971) but also the tubular structures (outer diameter 170-210 $\AA$ ) observed in various lower fungi (e.g. Oomycetes) and several algal groups (Chrysophyceae, Haptophyceae, Xanthophyceae, Phaeophyceae) which have been cyto- 
chemically identified as glycoproteins (Mignot et al., 1972). According to the hypothesis of Bouck (1969), these are secreted exocytotically by membrane flow to come to lie on the outer side of the plasma membrane surrounding the tinsel flagellum where they constitute the typical "flimmer" mastigonemes (Bracker et al., 1970; Bouck 1972; Heath et al., 1970; Leedale et al., 1970). Larger tubules (300-400 $\AA$ ) were noted in the perinuclear cisterna of the oenocytes of the migratory locust (Cassier and Fain-Maurel, 1968).

The indications that the perinuclear cisterna contains secretory proteins (e.g., serum albumin, specific antibodies, mastigonemes) leads to the question of whether the nuclear envelope contributes to vesicle flow processes which are known to be the basic mechanisms of intracellular translocation and secretory discharge. Indeed, there are many examples of regions of the nuclear envelope which have a "smooth" character (i.e., they are not charged with ribosomes) and show structures suggesting a vesicle pinching-off activity (Fig. 9). Such sites of bleb formations from the outer nuclear membrane, which often lie against juxtanuclear dictyosomes, have been reported in such diverse cell types as various Chromophyta and chloromonads, in various fungi, in microspore cells of gymnosperm plants, in cat myocardial cells, in rat adrenal cortex, in echinoderm oocytes, in gas gland cells of the perch fishes, in the subcommisural organ of the mouse, in avian pancreas, oviduct cells and oocytes, in mesodermal and lymphatic cells from embryonic chicken, in human neutrophilic granulocytes, in plasma cells, in mammalian bladder and intestinal epithelium, in ascites and myeloma tumor cells, and in early embryonic cells of insects and mammals (Zeigel and Dalton, 1962; Moore and McAlear, 1963; Bouck, 1965; Stoermer et al., 1965; Weston et al., 1965, 1972; Schnepf and Koch, 1966; Falk, 1967; Falk and Kleinig, 1968; Grove et al., 1968, 1970; Kessel, 1968b, 1971; Fawcett and McNutt, 1969; Longo and Anderson, 1969; Massalski and Leedale, 1969; Aldrich and Vasil, 1970; Herrlinger, 1970; Kilarski and Jasinski, 1970; Phillips, 1970; Schjeide et al., 1970; Heath and Greenwood, 1971; Franke, 1974; for further references see the reviews of Whaley, 1966, and Morré et al., 1971a). This vesicle production is particularly conspicuous in those algae and lower fungi in which the dictyosomes of the Golgi apparatus are confined to, or are at least accumulated at, the nuclear surface, with their "forming face" oriented toward the nucleus (or "proximal pole"; for terminology see Morré et al., 1971a; Whaley et al., 1971). It is exactly these dictyosome-adjacent regions of the nuclear envelope which often show a high vesicle-blebbing activity (Fig. 9) and appear in these organisms to be important loci of membranogenesis (or membrane turnover) and vesicle formation (Kessel, 
1971; Morré et al., 1971b). The morphology suggests that these vesicles become incorporated into the Golgi membranes and transport intravesicular secretory material from the nuclear envelope into the dictyosome. One can observe different types of vesicles blebbing off from the outer nuclear membrane: small, smooth-surfaced ones, larger multivesicular bodies (Kilarski and Jasinski, 1970), as well as vesicles with a typical bristle-coat. A dramatic transfer of vesicles from the nuclear envelope directly into the plasma membrane has been suggested during the formation of ascospore-delimiting membrane (Carroll, 1967; see also Beckett and Crawford, 1970, and Wells, 1972).

Association of vesicles with nuclear pore complexes has also been noted in various cells (Schjeide et al., 1970; Kiermayer, 1971; Franke et al., 1971b) and has been speculated to be the result of a pore-formation event ( Franke et al., 1971b; Franke, 1974).

In most cells, the contribution of membrane and secretory material from the nuclear envelope might quantitatively be of minor importance, compared to that produced in the ER. This is especially likely for those cell types in which an extensive rough ER is present. Such a minor contribution of nuclear membrane to the total secretory membrane flow is also indicated in biochemical studies using in vivo pulse-chase labeling of membrane protein in rat liver (Franke et al., 1971a). However, the situation might well be different in those cell types which have only little rough ER such as many of the above-mentioned lower fungi and algae. In these organisms, the nuclear envelope can be expected to structurally and functionally represent a prime source of vesicle flow and secretory activity. There is no indication so far that the nuclear envelope also participates in the synthesis of storage or cell wall polysaccharides. Activities of nucleoside diphosphate sugar transferases are very low in nuclear fractions. Mignot (1965), however, has observed within inflated parts of the nuclear envelope of the cryptomonad, Cyathomonas truncata, large granules positively stained with the periodic acid-Schiff reaction and has tentatively interpreted them as polysaccharide related to glycogen.

In summary, one can describe the importance of the nuclear envelope as a sort of a "minimum ER" of a eukaryotic cell. This is perhaps most clearly demonstrated in the mature avian erythrocyte, in which only remnants of ER are present, but in which the nuclear envelope constitutes about half of the total cellular membrane material. In this cell, typical ER activities such as $\mathrm{NADH}$-cytochrome $\mathrm{C}$ reductase or the enzymes capable of the incorporation of fatty acids into phospholipids are recovered exclusively in, and are confined to, the nuclear envelope (Zentgraf et al., 1971; Stadler and Franke, 1973). 
One can state that hitherto no general and basic differences between the ER and the nuclear envelope membranes have been described. A few properties which are distinct for the nuclear envelope in most cells do not hold in all. DNA attachment at the nuclear envelope is clearly not present in some special situations such as most of the nuclear envelope area during late meiotic stages, in the nuclear residues devoid of their chromosomes which are left after sperm formation of iceryine coccids (Moses and Wilson, 1970), and in the delaminated or foldedback regions of the nuclear envelope in some forms of spermiogenesis (see below). The occurrence of pore complexes is also not a reliable indicator of nuclear membranes in all cells, since they also occur in intranuclear and cytoplasmic annulate lamellae (AL), and occasionally even in normal ER as well (Kessel, 1968a; Wischnitzer, 1970; for pore complexes in single ER cisternae see Hoage and Kessel, 1968; Franke et al., 1972a; Orci et al., 1972). They are lacking, on the other hand, in some nuclear envelopes such as reported in sea urchin sperm (Longo and Anderson, 1968), in the micronucleus of a ciliate (Jenkins, 1967), * in mature mouse oocytes (Szollosi et al., 1972a), in the head part of mammalian sperm cells (below), and, according to Soyer (1969a), possibly also in the spores of the dinoflagellate, Noctiluca miliaris. Since the basic lipid and protein components seem to be so similar in the membranes of nuclear envelope and the $\mathrm{ER}$, it is interesting to note recent indications that the metabolic response of both membranes to cell physiological stimuli can show differences. While, in rat liver, glucose-6-phosphatase, for instance, shows a similar postnatal induction curve for both membranes (Kartenbeck et al., 1973), phenobarbital and other drugs stimulate the NADPH-cytochrome $\mathrm{C}$ reductase-containing redox system only in the $\mathrm{ER}$, not in the nuclear envelope (Jarasch, 1969; Kasper, 1971; for details see Chapter 6).

Some authors have argued that only the outer nuclear membrane has ER character but that the inner one is different (Kashnig and Kasper, 1969; Stevens and André, 1969; Ben-Porat and Kaplan, 1971; Kasper, 1971; Zbarsky, 1972b). Arguments for this view are (a) It is the outer membrane that is in direct continuity with the ER; $(b)$ the activities of some ER marker enzymes are lower in total nuclear membrane fractions; $(c)$ the inner membrane sometimes appears thicker and more heavily stained in electron micrographs of ultrathin sections; and $(d)$ differences in the lipid and protein patterns are observed between isolated nuclei before and after washing with detergents which are believed

"Micronuclei of other ciliates, however, have normal pore complexes (Tucker, 1967; Jurand and Selman, 1970; Stevenson and Lloyd, 1971; Stevenson, 1972). 
by some authors to selectively remove the outer nuclear membrane. However, none of these arguments is really stringent for the following reasons. ( $i$ ) There is usually much more continuity between inner and outer nuclear membrane via the pore walls than there are continuities between outer membrane and ER. ( $i i)$ Quantitative differences in membrane marker enzyme activities between ER and the nuclear envelope could as well represent "true" differences. Moreover, at least the glucose6-phosphatase in rat liver has recently been demonstrated to be located in both membranes (Kartenbeck et al., 1973). (iii) In most cases the greater thickness or stainability of the inner nuclear membrane is either limited to small specific sites of interaction with nucleoprotein or microtubular structures, or is due to a layer of electron dense nuclear material apposed to the inner nuclear membrane (see below). (iv) The detergents used do not specifically remove the outer membrane but rather progressively solubilize the lipoproteins of both membranes. They might select for binding strength of individual membrane components rather than for one whole membrane leaflet. Consequently, there is so far no reason to conclude that there exist true differences between the membrane leaflets as such.

Attempts to separate inner from outer nuclear membrane by fractionation have also been mentioned in the literature, but the results published do not yet permit one to conclude that the separation was adequate (Zbarsky et al., 1967; Smith et al., 1969; Mizuno et al., 1971a, b; Zbarsky, $1972 \mathrm{~b})$. In fact, during most fragmentation treatments the nuclear envelope preferentially breaks into vesicles comprising both inner and outer membrane material, rather than into sheets of either the inner or the outer membrane.

As is common for all ER cisternae, the luminal space of the nuclear envelope also appears variable in width from cell type to cell type after chemical as well after freeze fixation, and tends to swell under various inappropriate conditions (Fig. $21 \mathrm{~h}-\mathrm{j}$ ). An influence of the temperature of fixation, for example, has been noted particularly for late stages in avian erythropoiesis (Franke et al., 1973a). In some cell types, the two nuclear membranes are especially close together over the total nuclear surface, or only in localized regions, leaving an intracisternal width of ca. $100 \AA$ or even less. Narrow perinuclear spaces can be found, for instance, in the primary and secondary nuclei of Acetabularia, in various spermatids and mature sperm cells (especially at those membrane regions which lie adjacent to the acrosomal cap or to the centriolar base) and, in meiotic prophases, in those regions to which the synaptinemal complexes or the nucleoli are attached (Figs. 2b, c, 15b, 28, 35, 37; for literature see Section IX). In most cells, however, the relative equi- 
distance between the two nuclear membranes is a striking structural feature. It appears that the upright pore walls are sites of increased cisternal stability and counteract any forces separating the two membranes (see Fig. 21h and i; e.g. Monroe et al., 1967; Pankratz, 1967; Scheer and Franke, 1969; Comes and Franke, 1970). In addition, however, one frequently notes thin filamentous bridges linking the two cisternal surfaces of the inner and outer nuclear membrane (see Fig. 21j), and it has recently been suggested that such intracisternal bridges might play a role in the maintenance of the defined nuclear membrane spacing in a manner similar to that which has been discussed for other cytological situations of parallel membrane-membrane associations, including ER and Golgi cisternae (Franke et al., 1971c, 1972b, 1973a).

A cytological question often raised is whether the ER and the nuclear envelope are in direct membrane continuity with the plasma membrane; in other words, whether the intracisternal space of the nuclear envelope has direct, though perhaps only transient, connection with the extracellular space (McAlear and Edwards, 1959; Robertson, 1959, 1964; Buvat, 1963; Aldrich and Vasil, 1970; Carothers, 1972a, b). Along with other authors (Fawcett, 1964), we are sceptical, knowing of no unequivocal demonstration of a continuity between ER or nuclear envelope and the plasma membrane, although a very close association of both sometimes can be seen. The recently published micrographs of Carothers (1972a, b) and Aldrich and Vasil (1970) of androgonial and microsporogenetic cells of a liverwort and a gymnosperm have been interpreted as representing a particularly clear case of such a continuity. However, in such cells the plasma membrane tends to break down during fixations and to undergo myelinization, and the question of a possible artifactual origin of such membrane continuities by membrane breakage and fusion processes distinctly remains. A strong argument against communication of intracisternal spaces of ER and nuclear envelope is found in the series of observations that extracellular electron-opaque particles or materials have ready access to even the deepest and finest invaginations of the plasma membrane but are strictly excluded from the ER-nuclear envelope cisternal system. This has been a reliable criterion for distinguishing between the sarcolemma-bounded transverse channels and the accompanying sarcoplasmic reticulum cisternae or tubules, for identifying the demarcation membrane system in megakaryocytes (Behnke, 1968), and the tubular system of gastric glands and chloride cells (Ritch and

\footnotetext{
'In fact, the micrographs published in Carother's articles reveal some vesiculation and myelinization at the plasma membrane; Fig. 9 in the article of Aldrich and Vasil does not show clear continuities since the plasma membrane is obliquely sectioned.
} 
Philpott, 1969; Sedar, 1969). It might be, however, that secretory tubules, morphologically resembling smooth ER but being in a membrane-differentiation state homologous to that of secretory vesicles, transitorily fuse with the plasma membrane at the moment of exocytosis (Forte et al., 1969; Sedar, 1969; Whaley et al., 1971; see, however, also Hönigsmann and Wolff, 1973).

\section{Associations of Inner Nuclear Membrane with Chromosomes and Chromatin}

Not only is the inner aspect of the nuclear envelope exposed to the nucleoplasm but it also shows, in many cell types, a characteristic intimate and stable association with chromosomes or parts of them. In most interphase nuclei, an accumulation of condensed chromatin (the "heterochromatin" or "inactive chromatin" of the biochemical literature) is found in the nuclear periphery (Figs. 7a, 10,11, 16b, and 21f). In some nuclear types, a thin layer of condensed chromatin lining the nuclear envelope is the only remarkable condensed chromatin found at all. This intimate association of condensed chromatin is regularly interrupted at the pore complexes and the nucleoplasmic "channels" which lead into the pores (Figs. 7a, 16b, 20b and c; the "intranuclear channels" of Watson, 1959). In general, the chromatin association appears to be restricted to interpore sections of the nuclear envelope. A deoxyribonuclease sensitivity of this inner-membrane chromatin layer has been reported in a cytochemical study in a diatom, Streptotheca thamesis (Esser, 1968). Frequently, the outermost layers of this condensed chromatin reveal a highly regular substructural composition. Very intensely stainable and dense chromatin granules (150-200 $\AA$ in diameter) are arranged into strings and sometimes appear, in very close packing, even as rods or tubes (Figs. 11a and b; Davies 1967, 1968; Davies and Small, 1969; Barton et al., 1971). It is this inner nuclear membrane-attached chromatin which is important for maintenance of the specific nuclear shape (Franke and Schinko, 1969) and which is most resistant to swelling and extraction (Figs. 1le and $\mathrm{f}, 13 \mathrm{f}$ and $\mathrm{g}$ ). The structures observed during progressive swelling suggest that the granules are terminal coils of the 100-200- $\AA$ chromatin fibrils which could individually be traced for up to $0.4 \mu \mathrm{m}$ into the nuclear interior (Fig. 1le and $\mathrm{f}$ ). One could question whether this association is loose, fortuitous, and perhaps simply a consequence of the close proximity of chromatin and the envelope membrane. This seems to be ruled out, however, by the series of findings that isolated nuclear membranes contain DNA, al- 

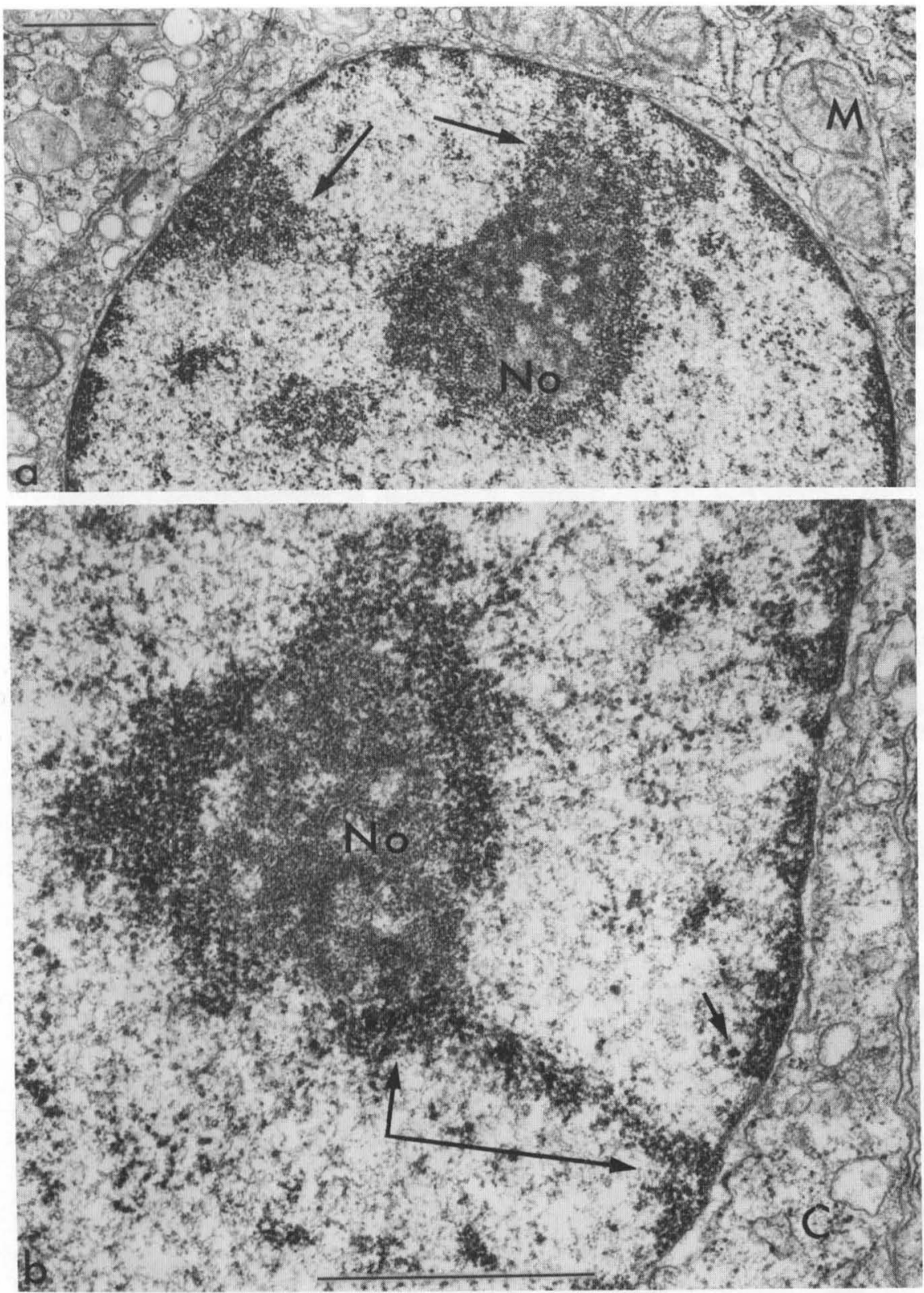

(10)

Fig. 10 Cross sections through the nuclear periphery of a Morris hepatoma cell, strain 5321 te (for preparation see Franke et al., 1971a). Note the accumulation of condensed chromatin at the nuclear envelope and its continuity with the perinucleolar heterochromatin which is mediated by one or two heterochromatin "pedicles" (arrows in $\mathrm{a}$ and $\mathrm{b}$ ). The small arrows in b denotes a "perichromatin granule." No, nucleolus; M, mitochondrion; C, cytoplasm ( $\mathrm{a}, \times 17,000 ; \mathrm{b}, \times 35,000$, bars indicate $1 \mu \mathrm{m}$ ). 


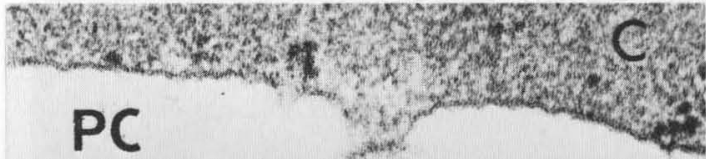
C.

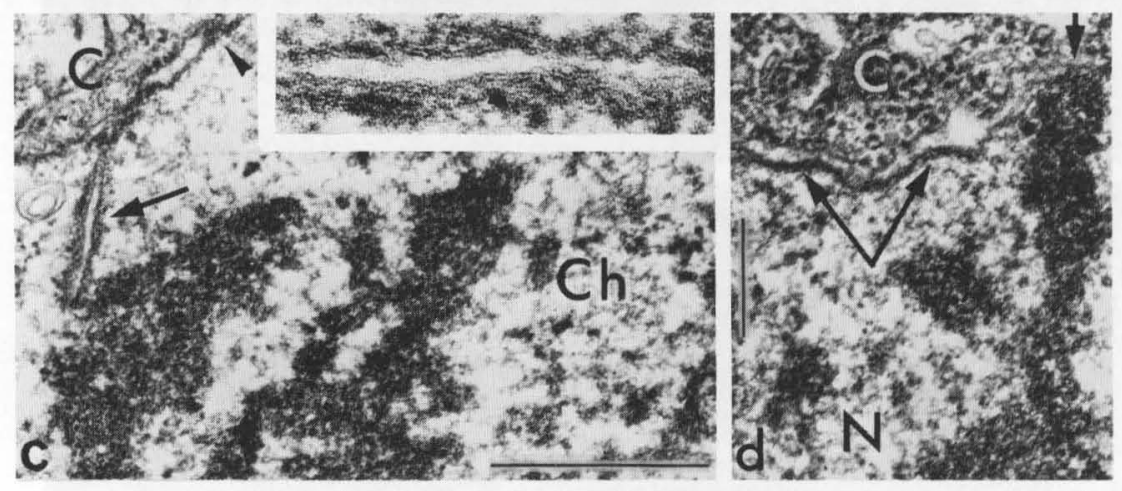

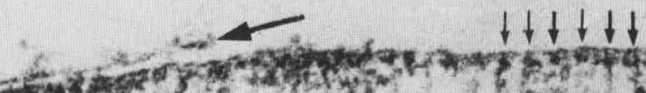

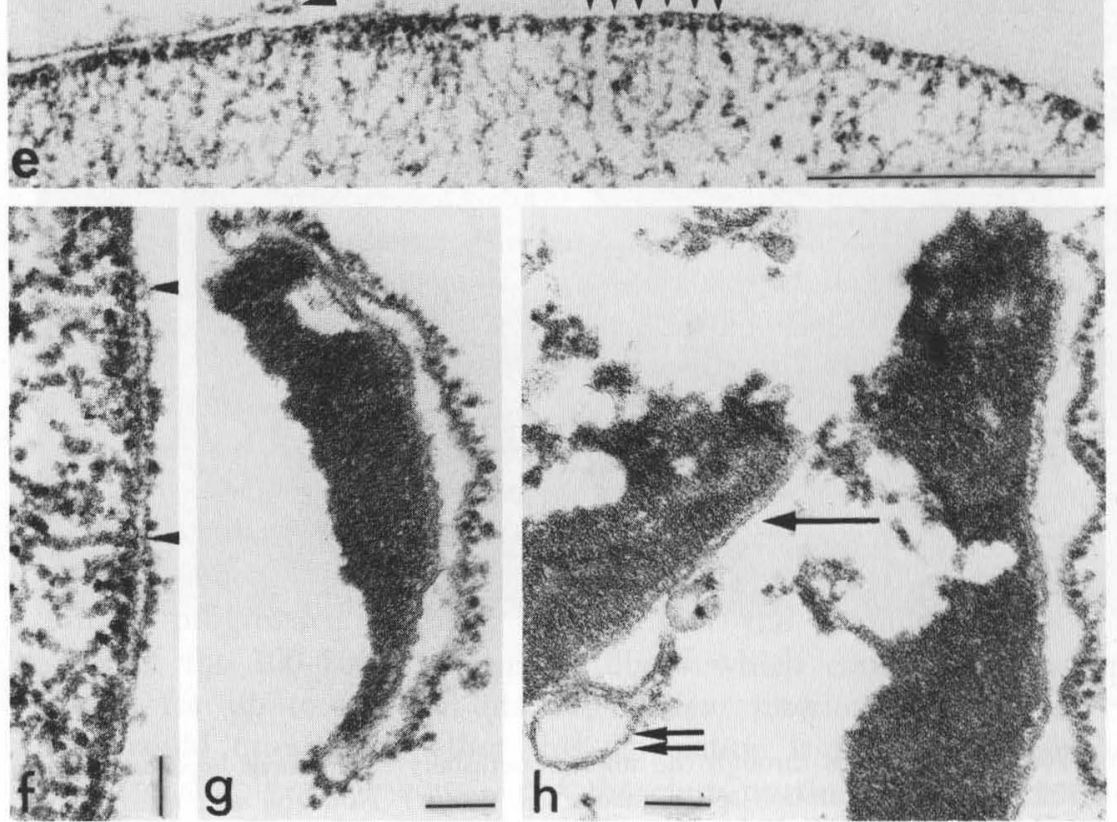

(11) 
though at proportions varying according to the specific isolation method (Zbarsky et al., 1969; Berezney et al., 1970, 1972; Franke et al., 1970; Kay et al., 1971, 1972; Zentgraf et al., 1971; Fakan et al., 1972; Monneron et al., 1972; Franke et al., 1973b; see, however, Kashnig and Kasper, 1969). Since Kubinski et al. (1972) have demonstrated that isolated ER and nuclear membranes are capable of in vitro binding of DNA and component deoxyribonucleotides, one must recognize as potential artifacts the random associations of nuclear DNA with the isolated membranes during the course of preparation. However, the association of nuclear DNA with the inner nuclear membrane is very firm, and seems to be a natural one from the following findings. ( $a$ ) The membrane-attachment of chromosomes or chromatin strands can be directly demonstrated in situ by cellular stratification (centrifugation; Mottier, 1899; Beams, 1948; Brenner, 1953; Pusa, 1963; Beams and Kessel, 1968; Beams and Mueller, 1970; see, however, the special case of the giant amoeba nucleus reported in the article of Wise and Goldstein, 1972) and by spreading preparations of nuclei and chromosomes (DuPraw, 1965; Comings and Okada, 1970a, b, c; Lampert, 1971; Stubblefield and Wray, 1971; Solari, 1972). It is also indicated by observations that the inner membrane is better preserved under mechanical stress or nuclear disruption (Fig. Ile and h), and that frequently the inner membrane is sepa-

Fig. 11 Various aspects of the associations of the inner nuclear membrane with condensed chromatin. In many nuclei the outermost layers of the peripheral condensed chromatin are arranged in rows of granular particles or in rods as seen in $a$ and $b$ in transverse and grazing sections in a hen erythrocyte (for fixation see Franke et al. 1973a; the small arrows in b point to the rows of granules). (c and d) Drosophila melanogaster salivary cells, showing the association of dense bands of giant chromosomes $(\mathrm{Ch}$ ) with the inner nuclear membrane ( $\mathrm{d}$, arrowhead in the upper right) or intranuclear cisternae (probably infoldings or derivatives from the inner nuclear membrane: c, arrow in the left; the arrowhead denotes a pore complex). This outermost chromatin layer is most resistant to solubilization and unraveling in low salt concentrations, as shown in e for a calf thymocyte nucleus, and in $f$ for a rat hepatocyte nucleus (arrowheads point to the terminal knobs of chromatin strands). The peripheral condensed chromatin appears to structurally stabilize the inner nuclear membrane leaflet, as suggested at sites where the outer membrane is lost (indicated by the arrow in the thymocyte nucleus of $e$ and the rat hepatocyte nucleus of $h$ ). The polarity of the attachment of deaxyribonucleoproteins and ribonucleoproteins to the perinuclear cisterna is illustrated in the isolated nuclear fragment from a rat hepatocyte, as shown in $\mathrm{g}$ and $\mathrm{h}$ : The outer membrane is densely set with ribosomes, whereas the inner one is intimately associated with blocks of condensed chromatin (the double arrow in $h$ points to a vesicle probably produced from the inner nuclear membrane). PC, perinuclear cisterna; $C$, cytoplasm; $N$, nucleus (a, $\times 88,000$; $\mathrm{b}, \times 80,000 ; \mathrm{c}, \times 44,000$; inset, $\times 145,000 ; \mathrm{d}, \times 60,000 ; \mathrm{e}$ and $\mathrm{f} \times 59,000 ; \mathrm{g}$, $\times 68,000 ; \mathrm{h}, \times 65,000$; bars in $\mathrm{a}, \mathrm{b}, \mathrm{f}-\mathrm{h}$ indicate $0.1 \mu \mathrm{m}$; in $\mathrm{d}, 0.2 \mu \mathrm{m}$; in $\mathrm{c}$ and $\mathrm{e}$ $0.5 \mu \mathrm{m})$. Compare e and $\mathrm{f}$ with Figs. 3 and 4 of Brasch et al. (1971). 
rated from the outer by its attachment to the peripheral chromatin (Franke and Schinko, 1969). A particularly instructive example is sperm development in the coccid, Steatococcus tuberculatus, in which the "chromatin (i.e., both chromosomes of this $n=2$ organism), attached to the envelope, is carried into the nuclear envelope papilla. . the envelope serving as a conveyer belt for the chromatin that accumulates on its surface" (Moses and Wilson, 1970). (b) Isolated nuclear envelope fragments show, in Kleinschmidt-type surface spreadings, DNA strands or loops hanging from them (Fig. 12; Franke et al., 1973b). Their morphology suggests that one molecule can have several attachment sites, in agreement with the garland models of Ormerod and Lehmann (1971; indirectly derived from sedimentation studies comparing, after detergent lysis, native and X-irradiation-damaged DNA) and of Comings (1968; see also Comings and Okada, 1970a), rather than with the alternative view that the membrane attachments are termini of individual DNA molecules. (c) A stable association with (membraneous) lipoprotein material has been noted in various chromatin preparations (BenPorat et al., 1962; Rose and Frenster, 1965; Jackson et al., 1968; Tata et al., 1972), and also with DNA during deproteinization treatments (Friedman and Mueller, 1968; Mizuno et al., 1971a, b; Hatfield, 1972). (d) The DNA-membrane association is resistant to centrifugation and high salt concentrations (e.g., $120,000 \mathrm{~g}$ in $4 \mathrm{M} \mathrm{CsCl}$ ), to the presence of hydrogen bond-disrupting agents such as $5.5 \mathrm{M}$ urea, to detergents such as sodium lauroyl sarcosinate (Sarkosyl), to shearing forces and sonication, and, to a small but significant extent, even to pancreatic deoxyribonuclease (Hecht and Stern, 1969; Franke et al., 1973b). This suggests that the anchor pieces of DNA at the membrane are firmly linked to, and protected by, membrane lipoproteins. The nature of the binding, however, is still unclear. $(e)$ The composition and the replication kinetics of the membrane-attached, purified DNA differ in some respects from the average nonmembrane-bound bulk nuclear DNA (Kay et al., 1971; Mizuno et al., 1971a, b; Deumling and Franke, 1972; Franke et al., 1973b).

Taken together, current evidence supports the view that the nuclear chromosomal DNA is constitutively attached to a membrane surface, similar to the genome DNA's of prokaryotes (for reviews see Jacob et al., 1963; Ryter, 1968; Tremblay et al., 1969; Jansz et al., 1971) and of mitochondria and plastids (Nass and Nass, 1963; Nass et al., 1965; Woodcock and Fernández-Morán, 1968; Bisalputra and Burton, 1969; 1970; Green and Burton, 1970; Odintsova and Turisheva, 1972; Sprey and Gietz, 1973). There exist numerous observations that attachment to the nuclear envelope is not random, but is limited to, or preferentially 


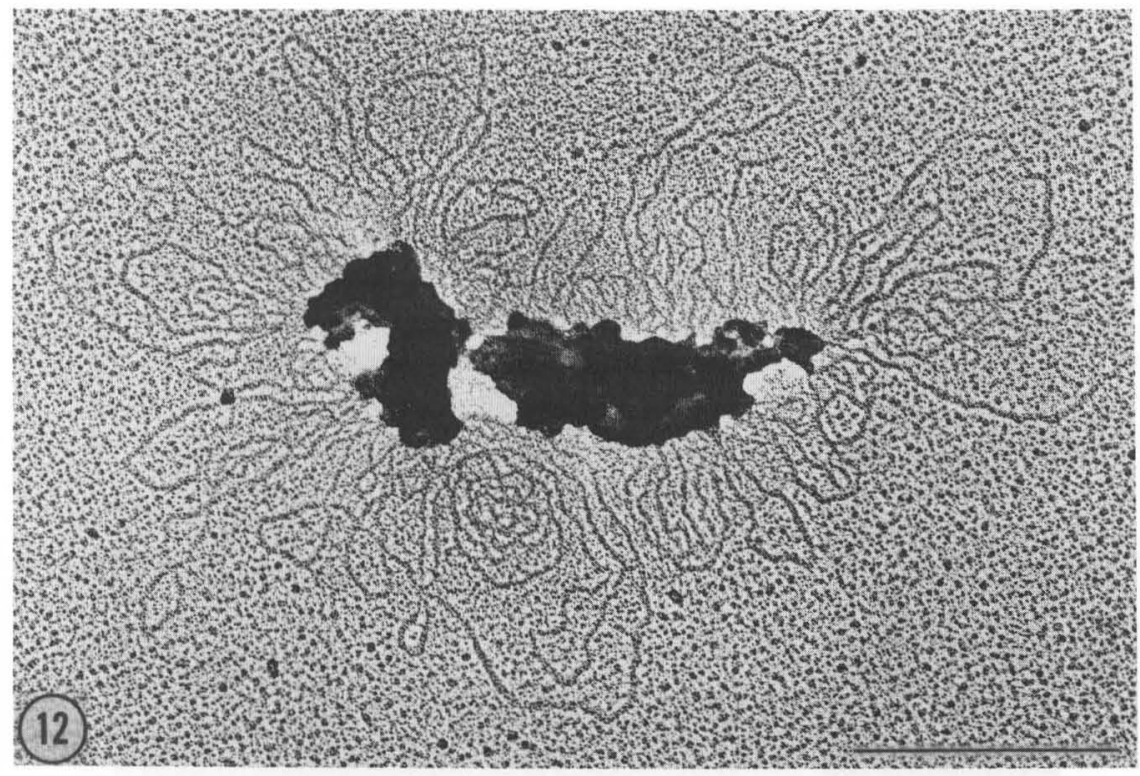

Fig. 12 Nuclear membrane fragment isolated from a hen erythrocyte after extraction with high salt concentrations and purification through both sucrose and $\mathrm{CsCl}$ gradient centrifugation (for details see Franke et al., 1973b) visualized in a surface spreading with cytochrome $\mathrm{C}$ (rotary-shadowed with platinum-palladium). Loops of DNA molecules are attached to the nuclear membrane $(\times 53,000$, bar indicates $0.5 \mu \mathrm{m}$ ).

occurs at, distinct chromosomes and chromosomal landmarks. Examples for such a regular and, at least in some cases, specific interaction are the mammalian sex chromatin body (Barr, 1959, 1960; Wolf et al., 1965), the W chromosomes in various snakes (Ray-Chaudhuri et al., 1971; Singh, 1972), the attachment of the nucleolar "pedicles" at the nuclear envelope (Figs. 10 and 13; Busch and Smetana, 1970; Rae and Franke, 1972), the attachment of centromere-equivalent regions in hypermastigid flagellates (Cleveland, 1938, 1957; Holland and Valentin, 1968a, b), and the attachment of dinoflagellate chromosomes at the basis of their $\mathbf{V}$ configurations (Leadbeater and Dodge, 1967; Kubai and Ris, 1969). Nuclear envelope adjacent heterochromatin also includes, or is enriched in, centromeric heterochromatin containing the specific satellite DNA's of the mouse and Drosophila flies (Figs. 13 and 14; Rae and Franke, 1972). In many organisms it is enriched in the heterochromatin which is demonstrable with the Giemsa-technique or with quinacrine fluorochromes (Fig. 13; Bianchi et al., 1971; Franke and Krien, 1972). In human cells the pericentromeric heterochromatin of chromosome number 

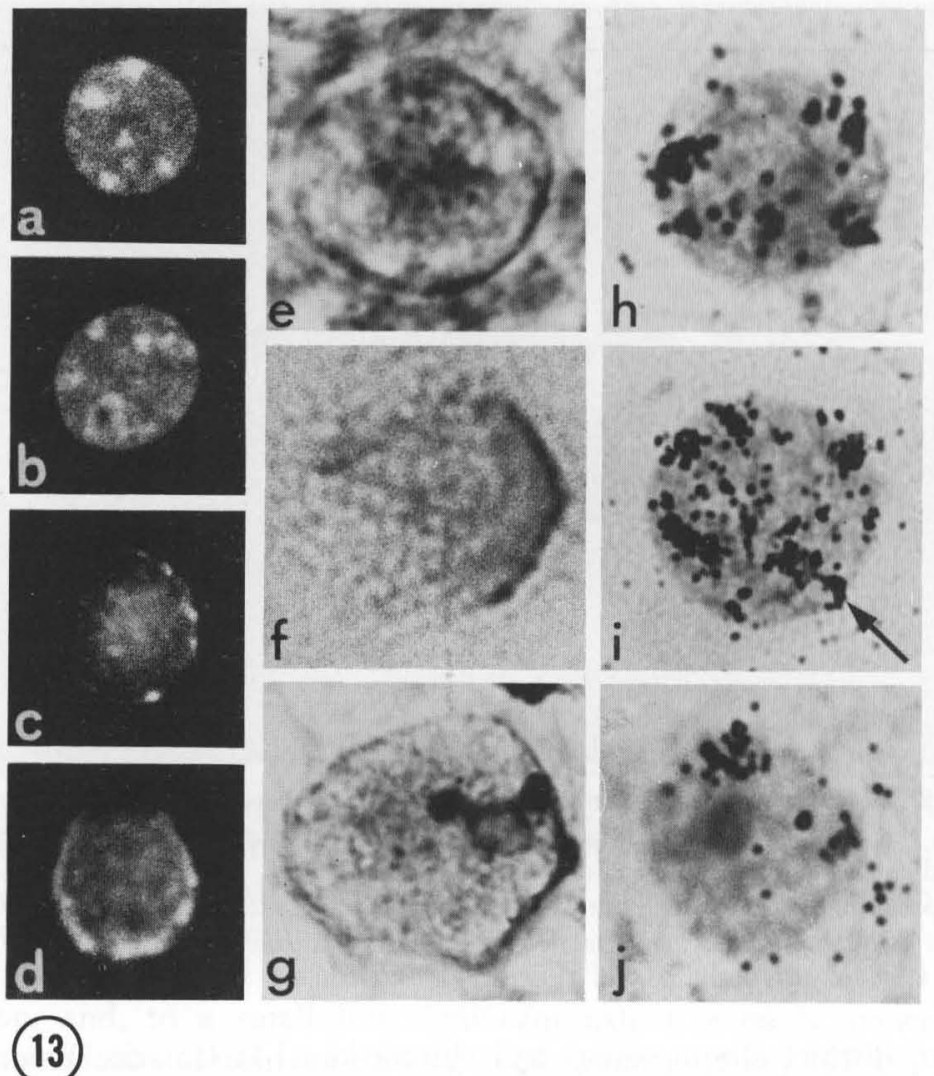

Fig. 13 Light micrographs showing various reactions of peripheral heterochromatin in nuclei of liver ( $a-f, h$ and $i$ ), Sertoli cells $(g)$, and spermatids $(j)$ of the mouse. The nuclear envelope-associated, condensed chromatin contains heterochromatin blocks which are demonstrable by quinacrine fluorochrome dyes ( $a-d ; 0.5 \%$ quinacrine- $\mathrm{HCl}$ in $96 \%$ ethanol), by the Giemsa technique (e-g; prepared according to Arrighi and Hsu, 1971; for details see Franke and Krien, 1972), and by in situ hybridization with tritiated RNA complementary to mouse satellite DNA ( $\mathrm{h}-\mathrm{j}$ : autoradiographs of $1 \mu \mathrm{m}$ sections; for details see Rae and Franke, 1972). Such peripheral heterochromatin is continuous with the perinucleolar heterochromatin (Figs. b, g, i), frequently through a stalklike connection (at the arrow in i). It is also the chromatin which is most resistant to extraction of nuclei with high salt concentrations ( $2 \mathrm{M} \mathrm{NaCl}$ or $\mathrm{KCl}$; Figs. c, d, f). Note that the pattern of distribution of such heterochromatin can vary from distinct, small individual blocks $(a-c, h-j)$ to large fused aggregate clumps (Figs. g, j), or to more uniform peripheral layers (d-f) which often are confined to one hemisphere (d and g: see also Hsu et al., 1971; Rae and Franke, 1972) (c, $\times 1300$; a, b, d, i, j, $\times 1500$; e, $\times 2200$; g and h, $\times 2300 ; \mathrm{f}, \times 2400)$. 
9 seems to be specifically attached to the envelope (Gagné and Laberge, 1972; compare Jones et al., 1973). It may also be that attachment of the dipteran giant chromosomes to the nuclear envelope (Fig. 11c and d) is preferential or specific for certain bands but this question requires further clarification.

A general orientation of centromeric regions onto the nuclear membrane has been noted by early cytologists, especially in studies of mitotic prophase (for reviews see Rabl, 1885; Strasburger, 1888; Heitz, 1932; Vanderlyn, 1948; Lettré and Lettré, 1959; Comings, 1968), and telomere associations with the nuclear envelope have also been discussed by some authors (Sved, 1966; Comings, 1968; Stubblefield and Wray, 1971). Prob-

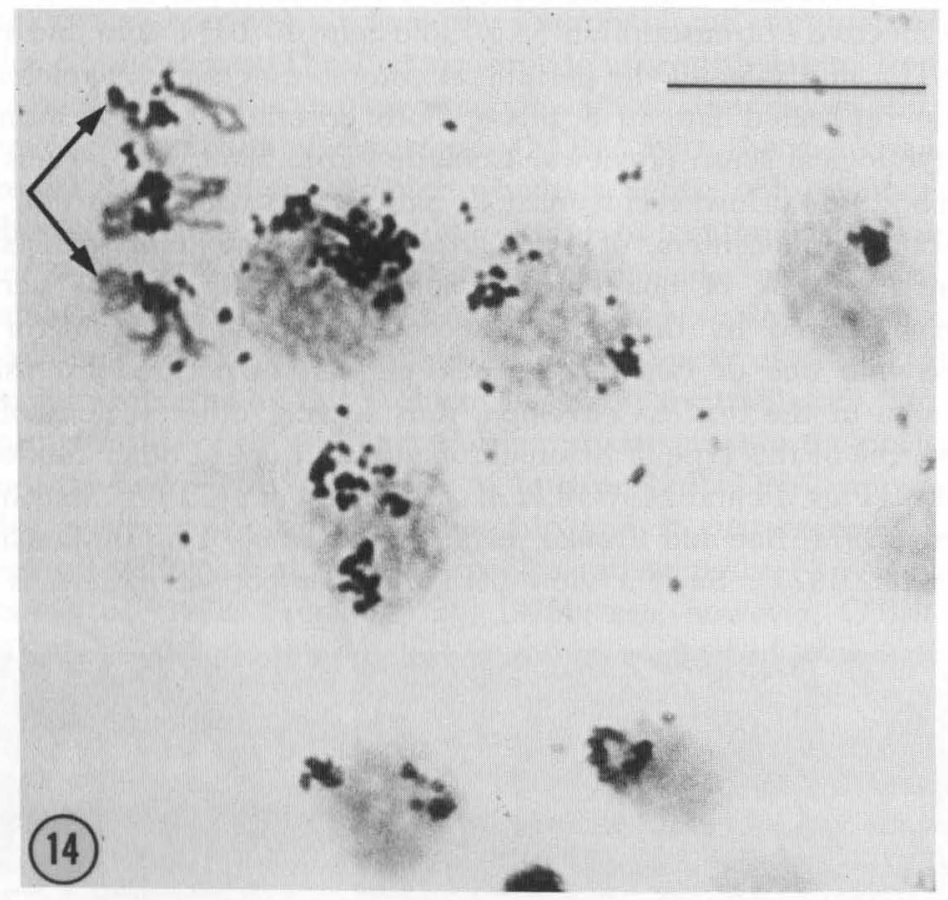

Fig. 14 Light micrograph showing the localization of the $\alpha$-heterochromatin of Drosophila melanogaster in interphase and metaphase chromosomes of ganglion cells, as revealed by hybridization in situ with tritiated RNA complementary to the $\alpha$-satellite DNA. This DNA which is located at the centromeres of metaphase chromosomes (denoted in the upper left by the pair of arrows) is contained in nuclear envelope associated chromatin blocks in the interphase nuclei, as demonstrated by the distribution of silver grains in an autoradiograph of a squash preparation. Such heterochromatin tends to fuse and sometimes exhibits an accumulation at one pole. $(\times 2600$, bar indicates $10 \mu \mathrm{m}$ ). (Courtesy of Dr. P. M. M. Rae, Yale University, New Haven.) 
ably the clearest case of an obligatory telomere attachment of chromosomes is observed during meiotic prophase, where termini of the synaptinemal complexes are firmly attached with their lateral elements to the nuclear envelope, an association which apparently is a prerequisite for the pairing of the homologous chromosomes (Fig. 15; Moses, 1960a; Pusa, 1963; Feldman et al., 1966; Wettstein and Sotelo, 1967; Woollam et al., 1966, 1967; Moses, 1968; Moens, 1969; Moens and Perkins, 1969; Comings and Okada, 1970b, 1972a; Hsu et al., 1971; Wettstein, 1971; Rimpau and Lelley, 1972; Solari, 1972). A special chromatin tab connecting an early pachytene chromosome to the envelope has been described by Roth and Parchman (1971) in the lily.

Moreover, nuclear membrane-associated chromatin has been shown to be very inactive in transcription (e.g. Goldstein, 1970a; Fakan and Bernhard, 1971), and some of the peripheral chromocenters are characterized by being replicated late in S phase, both criteria of heterochromatin in the cytological sense (reviews: Lima-de-Faria, 1959, 1969; Yunis and Yasmineh, 1971). However, it must be said in this connection that such peripheral chromocenters, or the peripheral chromatin in toto, is not constituted entirely of one kind of heterochromatin. This is perhaps best demonstrated by the fact that centromeric heterochromatin can be fused into one or two large blocks or can be located exclusively at one area of the nuclear envelope, thus leaving other regions of the envelope free of this type of heterochromatin, but not of other condensed chromatin (Figs. 13 and 14; Gall et al., 1971; Hsu et al., 1971; MacGregor and Kezer, 1971; Rae and Franke, 1972; Franke et al., 1973b; MacGregor and Walker, 1973). Such a view of a multicomponent pattern is also

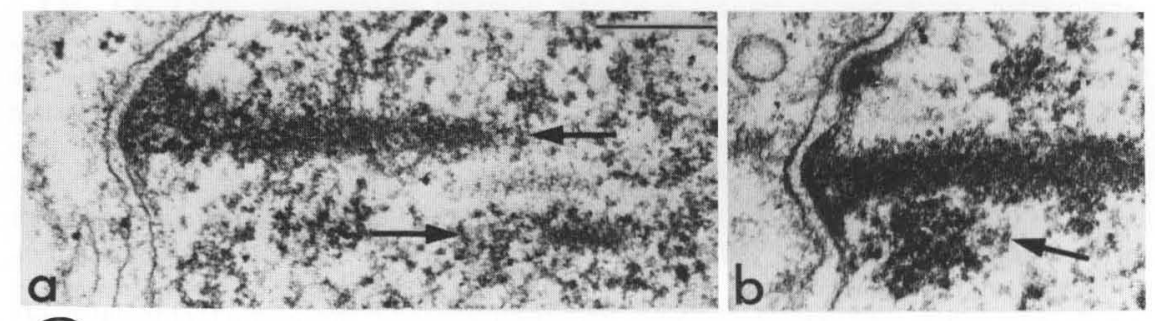

(15)

Fig. 15 Electron micrograph of the attachment of the termini of meiotic synaptinemal complexes to the inner nuclear membrane in a rat spermatocyte (for preparation conditions see Zentgraf and Franke, 1974). Note that membrane association is not only seen at the lateral elements (denoted by the arrows in a) but also at the apposed knobs of (telomeric?) heterochromatin (arrow in b). ( $\times 60,000$, bar indicates $0.2 \mu \mathrm{m}$ ). 
consistent with the results of biochemical analyses of purified membrane-attached DNA pieces, which show only a moderate enrichment of satellite DNA and other highly repetitive sequences (Franke et al., 1973b).

It has been suggested that membrane-attached DNA might contain considerable amounts of single strandedness (Mizuno et al., 1971b), but this view is in contrast to results obtained in our laboratory (Franke et al., 1973b ). Interesting in this context is the occurrence of supercoiled configurations in native membrane-attached DNA strands (Franke, Falk, and Zentgraf, unpublished observations ).

What could be the functions of this membrane attachment of chromosomal DNA? Two concepts are currently most discussed: $(a)$ a role in replication, and $(b)$ a role in the interphase and prophase orientation of the chromosomes. The first concept is an extrapolation from studies on replication of the prokaryotic genome to the eukaryotic nucleus, where the association of chromosomal DNA with the membrane should represent a replication complex which is active only at the onset of S phase (Comings and Kakefuda, 1968) or continuously through the entire S phase (Alfert and Das, 1969; Hanaoka and Yamada, 1971; Lampert, 1971; Mizuno et al., 1971a, b; Yoshida et al., 1971; YoshikawaFukada and Ebert, 1971; Hatfield, 1972; O'Brien et al., 1972). However, the results reported on this problem are highly contradictory. The kinetics of incorporation of thymidine and other precursors into the nuclear membrane-attached DNA, compared to nuclear bulk DNA, apparently excludes a precursor-product relationship and is characterized rather by an early leveling-off at a lower specific radioactivity (Kay et al., 1971; Fakan et al., 1972; Franke et al., 1973b; see, however, O'Brien et al., 1972). In addition, the nuclear membrane-attached chromatin shows a relative increase of incorporation of precursors in later stages of $\mathrm{S}$ phase (Blondel, 1968; Williams and Ockey, 1970; Erlandson and de Harven, 1971; Kay et al., 1971; Ockey, 1972; Franke et al., 1973b, and in autoradiographs, blocks of peripheral chromatin can maintain their label through several cell generations (Fakan et al., 1972; Franke et al., 1973b). This indicates that it is rather a special chromatin labelled in a special (late?) part of the $S$ phase and not a transitory state of the average chromosomal DNA. An interaction with the membrane seems also to be excluded in the replication of most of the bands of the giant chromosomes of Diptera, since they are not near the nuclear membrane. Moreover, in nuclei with a more chromonema-like chromatin distribution a preferential labeling of the periphery was not noted, even in late $S$ phase (e.g. Kuroiwa and Tanaka, 1971). In our opinion, the replicative behavior of the peripheral condensed chromatin is just another indication 
that it is predominantly (transcriptionally inactive) heterochromatin. While the existence of a specific nuclear membrane-bound DNA polymerase has been reported by some authors (Yoshida et al., 1971; Yoshikawa-Fukada and Ebert, 1971) it was not found by others (Deumling and Franke, 1972; Kay et al., 1972). The latter have emphasized that peripherally located nuclear DNA polymerase activity, judging from its solubility (in high salt concentrations, by prolonged DNase treatment, etc.) and enzymological characteristics, is common nuclear DNA polymerase bound to membrane-attached templates but not to the membrane itself.

Another question is whether membrane attachment of the chromosomes is a structural prerequisite for the initiation of their replication. There has so far been no case reported where a chromosome clearly not attached to the envelope is replicated (this holds also for the giant chromosomes). Pawlowski and Berlowitz (1969) studied the paternal heterochromatin ( $\mathrm{H}$ set) of the testis sheath cells of the mealy bug, Planococcus citri. They found that it is attached to the nuclear envelope although absolutely nonreplicating. This led them to the conclusion "that attachment to the membrane, in itself, is not sufficient to initiate or maintain DNA replication".

Another function of membrane-attachment of chromosomal material may be the orientation of chromosomes, or of special chromosomal regions, during interphase and mitotic and meiotic prophase (the possible function of the nuclear envelope in the course of intranuclear chromosome segregations will be dealt with in Section XII). It has been noted by many authors that specific parts of chromosomes are arranged during interphase and meiotic prophase in such a way that some chromosomal sites are associated with the nuclear membrane, preferentially the centromeric or pericentromeric heterochromatin or the telomeres (for reviews see Lettré and Lettré, 1959; Feldman et al., 1966, 1972; Comings, 1968; Feldman, 1968; Moses, 1968; Franke, 1974). As already mentioned, regular attachment of the telomeres (or the telomeric heterochromatin) to the nuclear envelope takes place at the termini of the synaptinemal complexes in the meiotic zygotene stage, and is important for the approach and pairing of homologous chromosomes (or homologous regions in different chromosomes). Similar associations have been discussed for the various forms of somatic pairing as being affected by the proximity of such regions at the nuclear envelope (the above references). In agreement with this view of a nuclear envelope attachment of distinct chromosomal regions are observations that the perinucleolar heterochromatin is regularly attached to the nuclear envelope in a variety of cell types (often in the form of the nucleolar pedicle; Fig. 10). The regularity of 
the nuclear envelope association with sex chromatin and the various kinds of heterochromatin mentioned above fits also into this view. Frequently one observes a pronounced polarity of the intranuclear distribution of a specific type of heterochromatin, for instance, the centromeric one (Gall et al., 1971; Hsu et al., 1971; Rae and Franke, 1972). In the cases of the mouse pericentromeric and of the onion late replicating heterochromatin this has been interpreted as indicating a maintainance of the telophase orientation during the interphase with the centromeric regions remaining aggregated at one pole at the nuclear envelope (Hsu et al., 1971; Fussel, 1972).

Further positional influence of the nuclear-chromosome interaction is recognized from the finding that chromosomal condensation always begins in the nuclear periphery. This holds for the normal condensations during mitotic prophase (Vanderlyn 1948; Comings and Okada, 1970d) as well as in experimentally induced ones (Robbins et al., 1970; Matsui et $a l .$, 1972) and might perhaps indicate that chromatin condensation is promoted by the existent condensed regions at the envelope.

From both the ultrastructure of kinetochores, especially those of the paired disc type (Comings and Okada, 1971; Luykx, 1970; Bajer and Molè-Bajer, 1972), and the notion that in various "primitive" forms of mitosis (hypermastigid flagellates, dinoflagellates, Phycomycetes, Zygomycetes, Ascomycetes; review: Pickett-Heaps, 1969) microtubule-organizing centers (MTOC) are located at the nuclear envelope, one could speculate that during evolution the centromere with its MTOC "originally situated on the nuclear envelope, becomes detached from it whilst retaining the ability ... to organize the spindle" (Pickett-Heaps, 1969). This would again fit with the whole concept of a localization of the centromeric chromosome regions at the inner nuclear membrane (see above) as well as with the idea of membrane-bound nucleating centers for microtubule assembly (see also Stadler and Franke, 1972).

It is not known whether the pattern and the stability of the chromosome-nuclear envelope attachment is constant throughout the whole interphase cell cycle. Changes of the DNA-nuclear membrane association during the cell cycle were recently suggested in a cytoimmunological study by Tan and Lerner (1972).

A very important question is that of the possible principles and modes of nuclear envelope formation or reconstitution, e.g., after mitosis in open nuclear divisions. One conclusion can clearly be made from the current data: The critical unit for organizing the formation of a nuclear envelope is not the entire nucleus but the individual chromosome or even parts of a chromosome. This is shown by the fully enveloped karyomeres (micronuclei) or individual chromosomes which are, for 
instance, observed in various meiotic divisions and after experimental chromosomal damage (Moses, 1960b; Harris, 1961; Thomas, 1964; Stevens, 1965; Schwalm, 1969; Stevens and André, 1969; Sachs and Anderson, 1970). This, as well as many observations that cisternal fragments of the prospective new nuclear envelope in late anaphase are first identified as being individually attached to the chromosomal surface, shows that the capacity for inducing the de novo formation of a closed envelope or for collecting preexisting cisternal fragments from the ER or from remainders of the "mother nuclear envelope" is a potential of the chromosome as such. It may be that nucleoproteins or lipoproteins located at the chromosomal surface serve as nucleating layers or centers for the assembly of membraneous lipoproteins or of cisternal fragments.

\section{Association of the Inner Nuclear Membrane with Specific Proteinaceous Layers}

In some nuclear types, especially very large ones, a honeycomb layer is intimately apposed to the inner nuclear membrane over the entire nuclear surface, with the combs precisely coaxial with the pores (two amoebae species: Harris and James, 1952; Greider et al., 1956; Pappas, 1956; Mercer, 1959; Daniels and Breyer, 1967; Stevens, 1967; Flickinger 1970; for reviews see Gall, 1964, and Stevens and André, 1969; gregarines: Beams et al., 1957; leech neurons and glial cell: Gray and Guillery, 1963; Coggeshall and Fawcett, 1964; Stelly et al., 1970; one green alga species: Burr and West, 1971). A similar, but thinner, and not so attractively structured, layer apposed to the inner nuclear membrane has been described in many other cell types (Fawcett, 1966; Kalifat et al., 1967; Mazanec, 1967; Patrizi and Poger, 1967; for further references see Stevens and André, 1969, and Stelly et al., 1970). In some instances a finely fibrillar substructure was noted within this layer; this led to the name "fibrous lamina" (synonyms: internal dense lamella, zonula nucleum limitans). In the authors' opinion, many other situations suggest the presence of a similar, though even thinner, densely staining proteinaceous layer associated with the inner nuclear membrane, a good example perhaps being the salivary gland nuclei of Drosophila (Fig. 11c and d; see also Berendes and de Bruyn, 1963). The cytochemical study of Stelly et al. (1970) strongly indicates that in neuronal nuclei such envelope-apposed layers consist almost exclusively of acidic proteins and do not contain considerable amounts of DNA or RNA. Although a role in the nucleocytoplasmic exchange and its control was discussed for some time as the chief function of such peripheral layers (Fawcett, 
1966a; Feldherr, 1968a) most authors now indicate that it merely serves as a supporting skeleton for the nuclear envelope (Fawcett, 1966a; Stevens and André, 1969; Flickinger, 1970; Stelly et al., 1970). However, this structure is a transitory one and not a feature specific for the nuclear type as such: In the amoebae, for instance, formation of the honeycomb layer after nuclear division takes place some time after the nuclear envelope has been reconstituted (Feldherr, 1966, 1968a, b), and in the alga Bryopsis, formation of the layer is confined to a defined stage of germling growth. It is important to note further that in such nuclei with inner membrane-apposed (protein) layers, the chromosomes are unlikely to be attached to the envelope, but rather to the material of this peripheral layer.

\section{The Pore Complex}

The characteristic structure of the nuclear envelope is the pore complex. Preceding the description of this structure, however, two essential restrictions of the generality of this sentence are necessary. First, there have been reports of nuclear envelopes totally devoid of pore complexes (for references see Section IV). Although some of these examples may simply be due to the rarity of pore complexes in the specific nuclear type, or to their obscurity in the specific electron microscopic preparation, one must think of the possibility that pore complexes are not inevitable structural features of the nuclear envelope. Second, the pore complex is not a structure occuring exclusively in nuclear envelopes: it is also found in special cytoplasmic and intranuclear cisternae, be they arranged into ordered stacks as typical $A^{\top}$, or isolated cisternae, including individual ER elements (see Section IV).

The ultrastructural organization of the pore complex has been the subject of a respectable number of studies, and there have also been substantial controversies of interpretation lasting for quite a long time (e.g. Afzelius, 1955; Dawson et al., 1955; André and Rouiller, 1956; Wischnitzer, 1958, 1960; Watson; 1959; Merriam, 1961; Gall, 1964; Nørrevang, 1965; Franke, 1966a, b, 1970a, 1974; Sichel, 1966; Vivier, 1967; Gouranton, 1969; Stevens and André, 1969; Abelson and Smith, 1970; Franke and Scheer, 1970a; Picheral, 1970; Roberts and Northcote, 1970, 1971; Blackburn, 1971; Engelhardt and Pusa, 1972; LaCour and Wells, 1972; Wunderlich and Speth, 1972; Fabergé, 1973). Recently, however, the majority of groups working in this field have reached essential agreement. The composition of the nuclear pore complexes has been studied by combinations of the principal electron microscopic techniques such 
as ultrathin sectioning, metal shadowing, negative staining, and deepfreeze fracturing, using material fixed in situ (chemically or by rapid freezing) or isolated nuclear membranes.

\section{A. The Pore Orifice Proper}

Pores are sites of fusion of the two cisternal membranes, in the case of the nuclear envelope the inner and outer nuclear membrane (Bahr and Beermann, 1954; Watson, 1954). The orifice of the pore complex, i.e., the lumen constituted by the pore walls, appears mostly circular in outline (Figs. 4, 6, 17-19; Watson, 1955; Merriam, 1961; Wood, 1966; Franke 1967a, b, 1970a; Millonig et al., 1968; Scheer and Franke, 1969; Franke and Scheer, 1970a; Neushul, 1970; Roberts and Northcote, 1970, 1971; Hanzely and Olah, 1973; LaFountain and LaFountain, 1973), although polygonal, especially octagonal, shapes have also been described (Gall, 1965, 1967; Kessel, 1969; Massalski and Leedale, 1969; Stevens and André, 1969; Abelson and Smith, 1970; Speth and Wunderlich, 1970; Maul, 1971a). There is still some debate concerning whether the circular or the polygonal shape represents the in vivo structure. Some authors interpret polygonal and various other noncircular outlines as artificial deviations from circularity and explain the predominant eight-sided form as resulting from the association of the pore periphery with the massive globular components distributed in an eightfold symmetry (see below) which would punctually stabilize the pore circumference during shrinkage and distortion processes (e.g. Franke 1970a, 1974; Franke and Scheer, 1970a; see, however, Maul, 1971a). The inner pore diameter is highly constant within a given nuclear type with a rather narrow distribution (e.g., $658 \pm 24 \AA \mathrm{SD}, 700 \pm 27 \AA, 737 \pm 67 \AA, 742 \pm 24$ $\AA, 713 \pm 23 \AA, 753 \pm 25 \AA, 723 \pm 28 \AA, 739 \pm 30 \AA$ in negatively stained, isolated nuclear envelopes from oocytes of various amphibia; Gall, 1967; Franke and Scheer, 1970a; for further data see the review articles listed above; also Branton and Moor, 1964; Comes and Franke, 1970; Kartenbeck et al., 1971; Thair and Wardrop, 1971). There seem to exist, however, some differences in mean pore diameters between different nuclear types (for instance, variations as reported from thin sections are from ca. $600 \AA$ up to ca. $1000 \AA$ ). Moreover, in the same cell, the mean pore diameter can be higher (sometimes up to $30 \%$ ) in freeze-etch preparations than in ultrathin sections or negatively stained preparations (compare Branton and Moor, 1964; Franke, 1966b; Speth and Wunderlich,

* Such values should not be confused with variations reported for inner and outer annulus diameters (Barnes and Davis, 1959; Thair and Wardrop, 1971). For dynamic changes in annular sizes (during muscle contraction) see also Bloom (1970). 
1970; Kartenbeck et al., 1971). Therefore, in measurements of the pore lumen diameter one has to discuss the possible alterations induced by the specific preparation method. For instance, the negative-staining method and tangential ultrathin sections can allow quite exact measurement of the luminal diameter, but both nuclear envelope isolation and the fixation and dehydration procedures frequently result in some shrinkage and distortion (Franke, 1970a, Speth and Wunderlich, 1970; Kartenbeck et al., 1971). Freeze fracturing, on the other hand, may better preserve the in vivo dimensions but it has the disadvantage that the fracture frequently leaves its plane in the membrane interior where the perinuclear space is relatively narrow (Kartenbeck et al., 1971). Consequently, in many nuclei freeze etching gives much larger values and a much broader distribution of inner pore diameters, since one sees fracture holes which spatially correspond to, but do not represent, pores (for details see Kartenbeck et al., 1971). Cisternal blebs or continuities with the ER must also be expected to lead to depressions in the replica which again can mimic pores. In addition, one can visualize a series of possible morphological changes artificially induced by the use of antifreeze agents, by the freezing process itself, and by the initial aldehyde fixation that has been used in most freeze-etch studies. However, pore diameter differences are not always found when different preparative methods are compared. In the nuclear envelope of amphibian oocytes, e.g., the pore size is the same after all three techniques, freeze etching, ultrathin sectioning, and negative staining of isolated envelope fragments (Scheer, 1970a, 1973; Kartenbeck et al., 1971).

\section{B. The Annulus Structures}

Both the inner (nucleoplasmic) and outer (cytoplasmic) rims of the pores are associated with nonmembraneous material (Figs. 16-30). This material, which accentuates the pore perimeter, appears as rings lying on top of either pore margin and has been called the "annulus" (for definition of this term see Callan and Tomlin, 1950; Afzelius, 1955; Gall, 1964; Franke, 1970a, 1974; Franke and Scheer, 1970a; Roberts and Northcote, 1970, 1971). The stainable, electron-opaque material constituting these rings is not a homogeneous doughnut but is arranged in (mostly) eight symmetrically distributed granular subunits with diameters between 100 and $250 \AA$ ("annular granules;" e.g., Figs. 16-19; Gall, 1954, 1956, 1964; Pollister et al., 1954; Watson 1955, 1959; Rebhun, 1956; Swift, 1958; Grimstone, 1959; Drawert and Mix, 1961; Merriam, 1961; Wartenberg, 1962; Jacob and Jurand, 1963; Werz, 1964; Nørrevang, 1965; Franke, 1966a, b, 1967a, b; Mentré, 1966, 1969; Monroe et al., 1967; Verhey and Moyer, 1967; Yoo and Bayley, 1967). The eightfold radial 


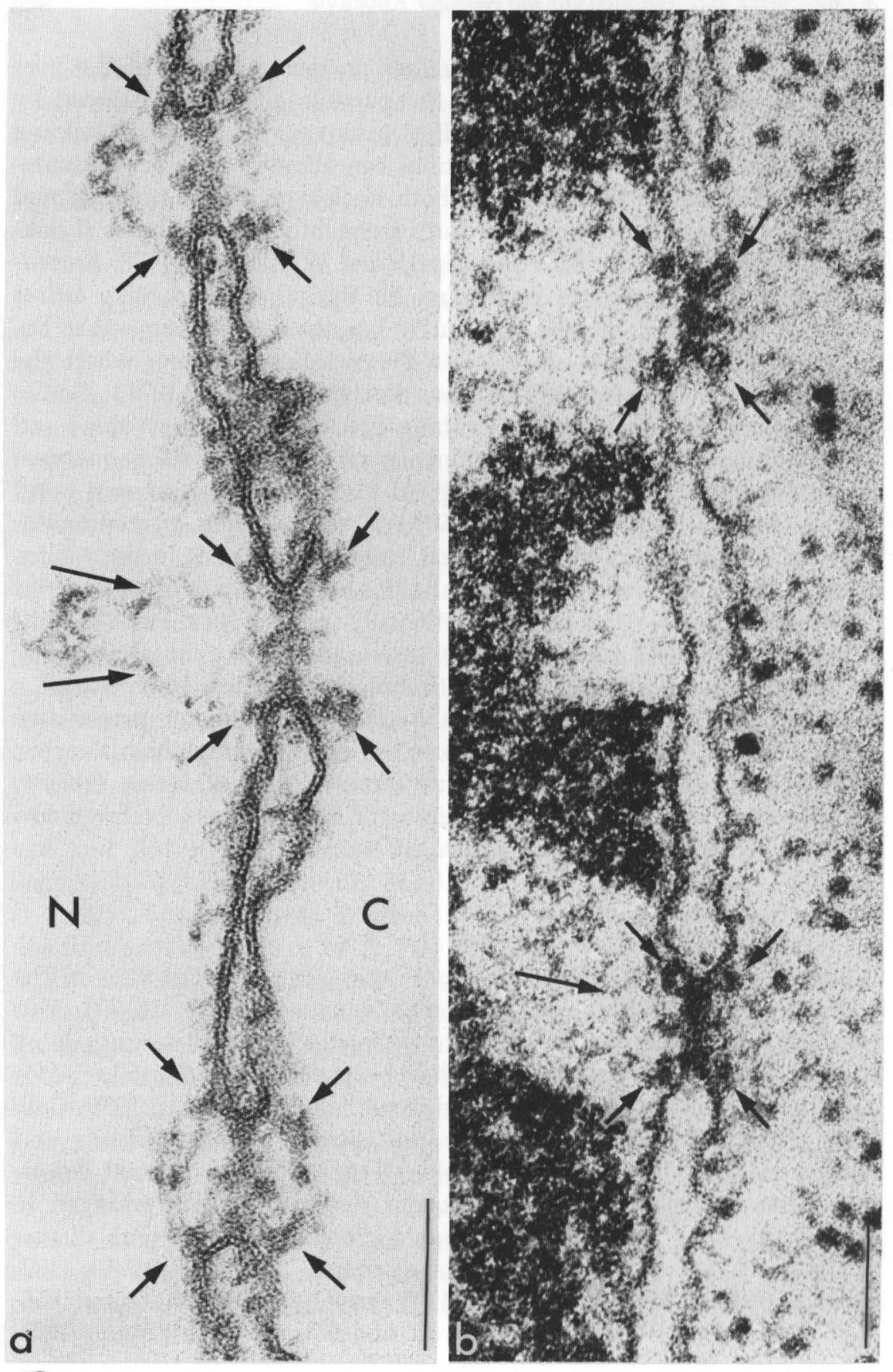

(16) 
symmetry of their arrangement was suggested by the observations of Watson (1959) and others (Merriam, 1961; Wolstenholme, 1966), then demonstrated by the pattern enhancement technique of Markham et al. (1963) for a variety of organisms (Fig. 19; Franke 1966a, b, 1970a; Fisher and Cooper, 1967; Franke and Kartenbeck, 1969; DeZoeten and Gaard, 1969; Comes and Franke, 1970; Franke and Scheer, 1970a; Hanzely and Olah, 1973; compare also Abelson and Smith, 1970). In some special, very instructive preparations, this pattern could be directly encountered in the micrographs (Figs. 17d, 18i, and 19; Daniels et al., 1969; Franke and Scheer, 1970a; LaCour and Wells, 1972; Fabergé, 1973). Although 8 seems by far to be the predominant number of granules within an annulus, exceptions of either 9 or 7 have also been noted (Franke, 1967b; Bajer and Molé-Bajer, 1969).

There are also suggestions from electron micrographs that the corresponding granules of either annulus are superimposed, i.e., aligned with each other. The centers of the annular granules are usually slightly outside of the pore perimeter proper (Fig. 19; Afzelius, 1955; Merriam, 1961). Annular granules are readily identified in thin section and negative-staining preparations (in addition to the aforequoted references see Mepham and Lane, 1969; Flickinger, 1970; Herrlinger, 1970; Roberts and Northcote, 1970; Burr and West, 1971). They are less frequently seen in freeze-etch replicas (see Moor and Mühlethaler, 1963; Branton and Moor, 1964; Franke 1966b, 1970a; Stevens and André, 1969; Scheer, 1970a; Speth and Wunderlich, 1970; for review see Kartenbeck et al., 1971), in which they are encountered only where the fracture jumps over the nuclear pore complex so that the annulus structures can be exposed by the subsequent etching process (Roberts and Northcote, 1970; Kartenbeck et al., 1971).

One frequently sees that the granules of the outer annulus are close to and in register with polyribosomes, be they spiral shaped and bound to the outer nuclear membrane, or helical ones extending from the pore

Fig. 16 Details of the nuclear pore complex organization as seen in transversely sectioned nuclear envelopes isolated (a, from maturing Xenopus laevis oocytes) or fixed in situ ( $b$, onion root meristem). The small arrows point to the annular granules lying upon either pore margin. Clumps of dense material project from the pore wall into its lumen. The center of the pores is often occupied by a distinct densely stained particle, the "central granule." The larger arrows denote nucleoplasmic fibrils terminating at the inner annular granules. Note, in b, the confinement of peripheral condensed chromatin to the interporous segments, thus leaving the "nuclear channels" which run onto the pores. Orientation is such that the nucleoplasmic side $(N)$ is to the left $(a, \times 200,000 ; b, \times 163,000$, bars indicate $0.1 \mu \mathrm{m})$. 

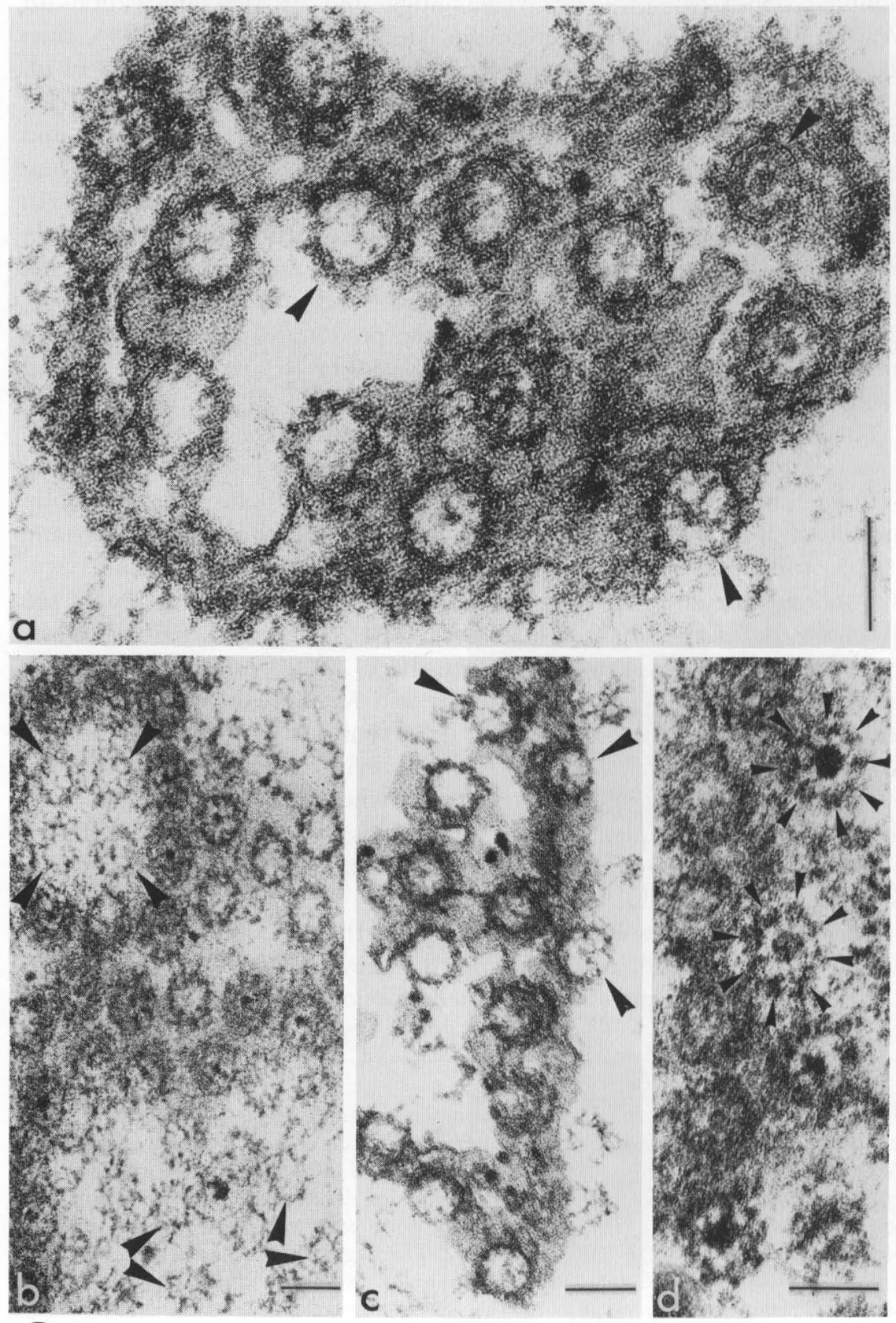
complex vicinity into the cytoplasm (Figs. 7b, 18a, 24d and e, 27, 30; Claude, 1964; Millonig et al., 1968; Mepham and Lane, 1969; Franke, 1970a; Franke and Falk, 1970; Jacob and Danieli, 1972). It could now be asked whether they represent ribosomes (Palade, 1955; Gall, 1956; Mentré, 1969). The annular granules, however, show some discrete differences from ribosomes: the annular granules appear in some preparations to be significantly larger (Fig. 21d and e; see also Jacob and Danieli, 1972; Franke, 1974) and less densely stained (Figs. 18a, 19a-j, and 30; Watson, 1959; Monroe et al., 1967; Franke, 1970a; Franke and Falk, 1970; Franke and Scheer, 1970a; Jacob and Danieli, 1972), exhibit a less dense core (Figs. 17, 18, and 30; Nørrevang, 1965; Rebhun, 1956; Watson, 1959; Franke, 1970a; Franke and Falk, 1970; Franke and Scheer, 1970a; Jacob and Danieli, 1972), and behave somewhat differently in cytochemical reactions (see below).

Thin fibrillar threads are connected with the granules of either annulus, especially the inner one (Figs. 16, 17b, 20, 21, 23, and 24; for references see DuPraw, 1965; Verhey and Moyer, 1967; Kessel, 1968b, 1969; Franke and Scheer, 1970a; Maul, 1971a; Engelhardt and Pusa, 1972). Nucleoplasmic fibrils, 30-70 $\AA$ in diameter, often studded with electrondense 50-120 $\AA$ granules at variable intervals, terminate at the inner annular granules (inner annulus-attached fibrils; Franke, 1970a; Franke and Scheer, 1970a). These fibrillar masses are firmly attached to pore complex material, as is demonstrable in isolated nuclear envelopes (Figs. $8,16 \mathrm{a}, 20 \mathrm{a}, 21 \mathrm{a}, \mathrm{b}, \mathrm{d}$, and $\mathrm{g}, 23 \mathrm{~g}$, and 24c; see also Millonig et al., 1968; Franke and Scheer, 1970a; Scheer, 1972). In some nuclei, nucleoplasmic fibrils terminating at one annulus constitute a cylindrical unit (Figs. 17b, 18a, and 20; Franke and Scheer, 1970a) which can be traced, e.g. in amphibian oocytes, for more than $0.5 \mu \mathrm{m}$. It is noteworthy that one fibrillar group is not exclusively associated with only one pore com-

Fig. 17 Nuclear pore complex substructures as revealed in sections tangential to nuclei which were isolated (a-c, from Xenopus laevis oocytes) or fixed in situ ( $\mathrm{d}$, primary nucleus of Acetabularia mediterranea). The dark-light-dark (unit membrane) pattern of the pore wall is identified (at the upper right arrowhead in a). Some of the pores contain a marked central knob (at the arrowhead in the upper right of a); others show threads radiating from such a central element (lower right arrowhead in a and the upper marked pore in $\mathrm{d}$ ); some are traversed by individual thin filaments, and some do not reveal any conspicuous internal structures at all (left arrowhead in a and the arrowheads in c). The eight granular subunits of the inner annulus are seen in $\mathrm{d}$ as well as their thread connections with the central granule. A grazing section (b) shows that the nucleoplasmic fibrils associated with an inner annulus are arranged into a cylindrical unit (some cross sections are indicated by the arrowheads) $(\mathrm{a}, \times 145,000 ; \mathrm{b}, \times 74,000 ; \mathrm{c}, \times 88,000 ; \mathrm{d}, \times 115,000$, bars indicate $0.1 \mu \mathrm{m}$ ). 

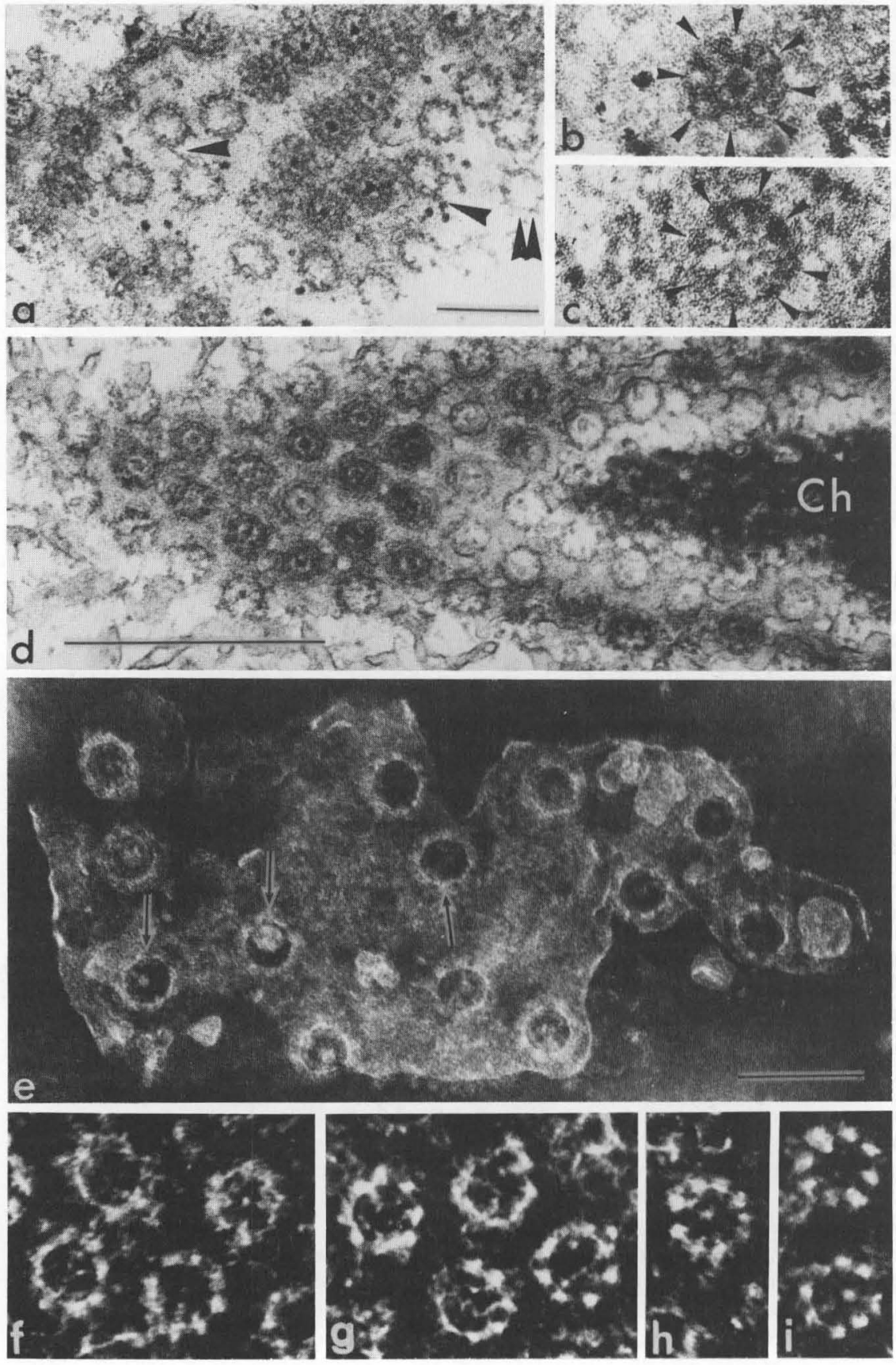

(18) 
plex: one can recognize "branching" of these pore-associated fibrillar cylinders, as well as situations where a fibrillar tangle is attached, at nuclear envelope invaginations, to two opposite pore complexes (Fig. 20a).

Depending on the specific preparation method and the cell type, the annular granules can appear somewhat loosely packed and suggest a fibril-coil substructural organization (e.g. Kessel, 1969; Franke, 1970a; LaCour and Wells, 1972; Wunderlich and Speth, 1972; Fabergé, 1973; for further references see Franke, 1970a, 1974).

Under appropriate preparation conditions the annular granules remain attached to the pore margins (Figs. 16a, 18f-i, 21a and b; Gall, 1954, 1956, 1964; Merriam, 1961; Franke, 1966a, b, 1967a, b; Franke and Scheer, 1970a). They tend to "unravel" and disappear, however, when stabilizing divalent cations are absent and in very low or high ionic strength conditions, thus facilitating visualization of the membraneous pore perimeter proper (Figs. 4-6; Franke and Scheer, 1970a; see also a remark of Callan and Tomlin, 1950).

\section{The Internal Structures of the Pore Complex}

In most nuclear pores the pore lumen is not entirely filled with the relatively electron-translucent ground cytoplasm, but contains regularly

Fig. 18 Further structural details of pore complexes as seen in tangential sections of in situ fixed material (a, Xenopus laevis oocyte, lampbrush stage; b, isolated rat hepatocyte nucleus; c, vegetative nucleus of a Lilium longiflorum pollen tube; d, maturing spermatid of Triturus alpestris) and in negatively stained, isolated nuclear envelopes (e, from HeLa cell; for preparation see Comes and Franke, 1970; f-i, from a nearly mature Triturus alpestris oocyte; for details see Franke and Scheer, 1970a). Central granules can be frequent in nuclei active in RNA synthesis (a) as well as in inactive nuclei (d). They are mostly not detected in pore complex images in which the equatorial plane of the pore is not included in the section (a). (a) Note fibrillar pore-to-pore connections (left arrowhead), ribosomes associated with pore complexes (arrowhead in the right), and cross-sectioned cylinders of annulusattached fibrils (double arrowhead). (b) The inner ring is connected to both the central granule and the pore periphery by threads, which are interspaced with eight symmetrically distributed, electron-translucent sectors (arrowheads). (c) The arrowheads point to the eight conical tips projecting from the pore wall. (e) The great size variability of the central elements (e.g., at the arrows in the left) which in some pores appear to be totally absent (right arrow). ( $f$ and $g$ ) Pore complexes with a variety of internal fibrillar formations. ( $h$ and i) The discontinuity of the annulus, i.e., the eight granular subunits ( $\mathrm{a}, \times 64,000$, bar indicates $0.2 \mu \mathrm{m} ; \mathrm{b}$, $\times 125,000 ; \mathrm{c}, \times 175,000 ; \mathrm{d}, \times 66,000$, bar indicates $0.5 \mu \mathrm{m}, \mathrm{Ch}=$ chromatin; $\mathrm{e}, \times 80,000$, bar indicates $0.2 \mu \mathrm{m} ; \mathrm{f}-\mathrm{i}, \times 125,000$ ). (The preparation shown in $\mathrm{b}$ was done collaboratively with Dr. R. Kay, University College, London.) 

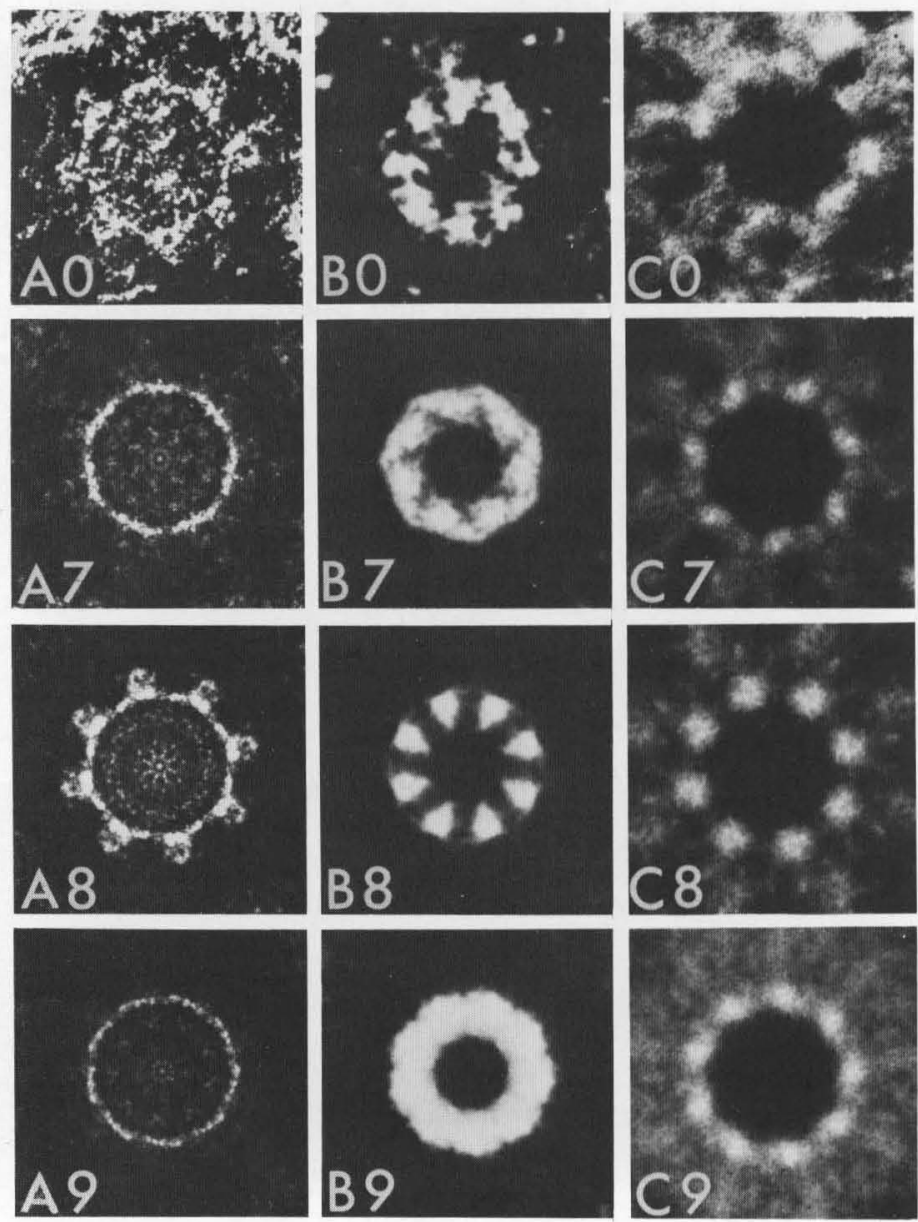

(19)

Fig. 19 In rotational analyses of negatively stained pore complexes using the multiple exposure technique of Markham et al. (1963), one observes the maximal image enhancement by superposition at eight rotations (A8, B8, C8), compared to other values of $n$ (examples for $n=7$ and $n=9$ are given here). This indicates that both peripheral components of the pore complex, the annular granules ( $\mathrm{A}$ is an example of a Triturus alpestris oocyte, $\mathrm{C}$ is from an onion root tip cell) as well as the projecting tips (peripheral granules, shown in an example from Triturus alpestris oocytes in B), are arranged in an eightfold radial symmetry. Note that the centers of the annular granules are outside of the pore perimeter (A0 and A8) ( $A$ and $\mathrm{B}, \times 175,000 ; \mathrm{C}, \times 200,000)$. 

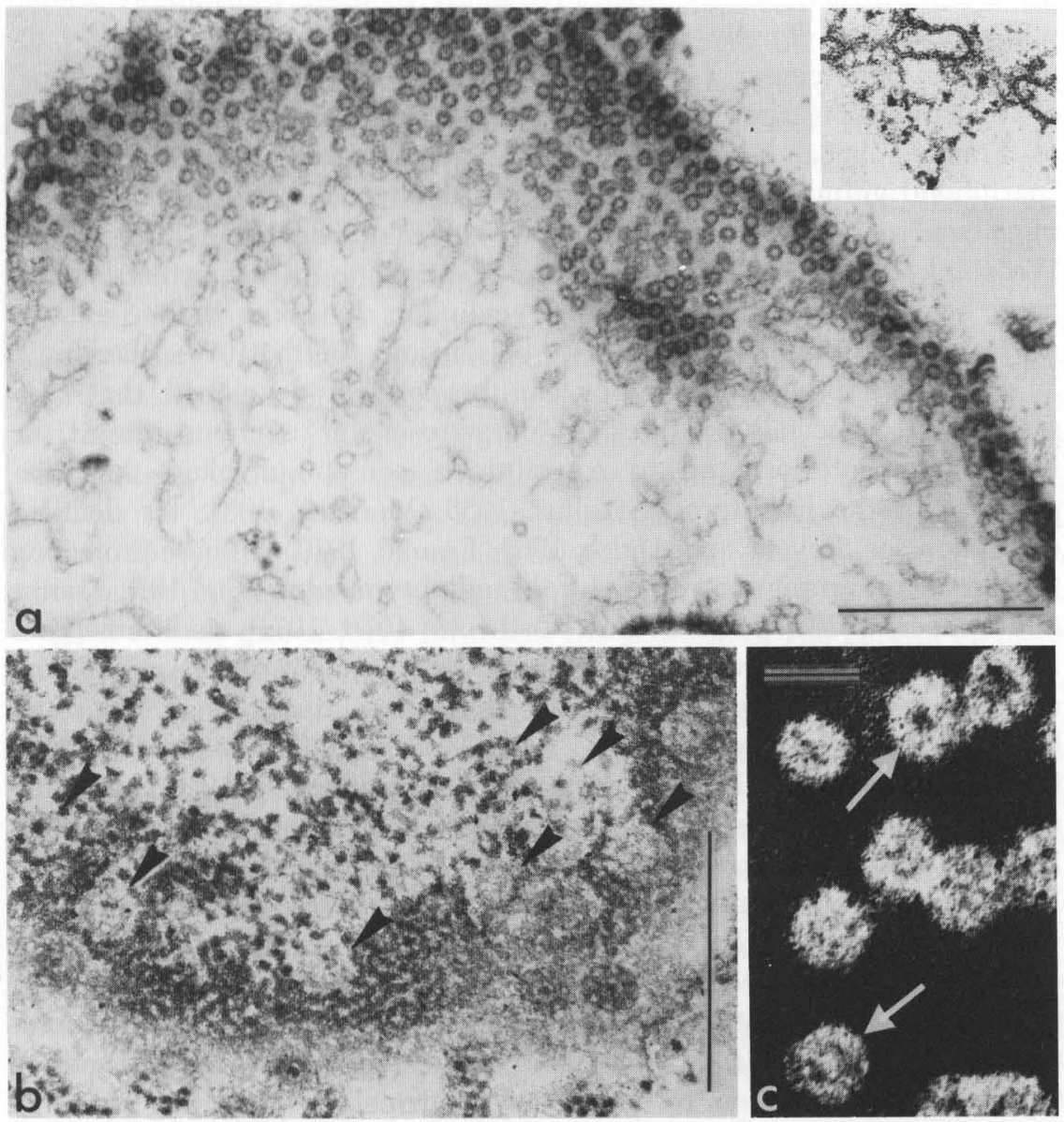

(20)

Fig. 20 Nuclear fibril formations terminating at the pore complexes are especially conspicuous structures in maturing amphibian oocytes (as demonstrated in a) in a grazing section (Triturus alpestris). The inset shows that fibrillar bundles attached to the inner annulus can be intermingled and fused. In nuclei with peripheral condensed chromatin ( $b$ and $c$ show isolated rat hepatocyte nuclei) such inner annulusattached fibrils run within the chromatin-free channels and can be visualized, in cross sections, as ring structures (arrowheads in b), many of them containing a central dense element. Note also the fine filamentous connections between the central element and the channel periphery (e.g., at the upper arrow in c) (a, $\times 22,000$, bar indicates $1 \mu \mathrm{m}$, inset, $\times 80,000 ; \mathrm{b}, \times 56,000$, bar indicates $0.5 \mu \mathrm{m}$; c, $\times 100,000$, bar indicates $0.1 \mu \mathrm{m}$ ). 
arranged, distinct substructures. Frequently the pore walls are associated with clumps of densely staining material which protrude conically toward the pore center (Figs. 16, 18c, 19B; the "fingerlike projections" of Watson, 1959; the "Zipfel", "projecting tips" or "tiplike projections" of Franke, 1966a, b, 1967a, b, 1970a; Franke and Scheer, 1970a; the "peripheral granules" of Roberts and Northcote, 1970, 1971; the "traversing fibers" of Maul, 1971a; the "claws" of Engelhardt and Pusa, 1972). We think that these projections are identical to the structures which have been envisaged as tubules or cylinders by some earlier authors (Wischnitzer, 1958; Vivier, 1967; Abelson and Smith, 1970; Blackburn, 1971). These projecting clumps can be so well developed that they seem to fill, especially in thick or "off pore center" sections, almost the entire pore interior, and to constitute a compound plug-the "diaphragm" of the literature (Afzelius, 1955; Merriam, 1961; for detailed discussion see Stevens and André, 1969; Franke, 1970a). These projecting tips are again arranged in an eightfold radial symmetry (Fig. 19B; Franke and Scheer, 1970a; Roberts and Northcote, 1970, 1971) and sometimes appear to be aligned with the corresponding granules of either annulus: a great many micrographs suggest that these tips project from the bases of the annular granules. As a consequence of their location deep in the pore, peripheral granules attached to the pore wall are commonly recognized in freeze-etch replicas of nuclear envelopes (Neushul, 1970; Roberts and Northcote, 1970; Speth and Wunderlich, 1970; Thair and Wardrop, 1971; Kartenbeck et al., 1971; Maul, 1971a; Teigler and Baerwald, 1972). As with the annular granules, the peripheral clumps can also exhibit fibril-coil aspects. One often notes that they elongate into thin threads which run onto the pore center, or connect the periphery with the central granule or the inner ring (see below). In various nuclear types these threads seem to be the only centripetally projecting elements and again can correspond in position to the annular granules (Figs. 17 and 18; Merriam, 1961; Vivier, 1967; Yoo and Bayley, 1967; Wunderlich and Franke, 1968; Daniels et al., 1969; Kessel, 1969; Abelson and Smith, 1970; Franke, 1970a; Franke and Scheer, 1970a; Howard and Moore, 1970; Roberts and Northcote, 1970, 1971; LaCour and Wells, 1972). The eightfold symmetry of such radiating threads has also been demonstrated (Daniels et al., 1969; Franke, 1970a; compare also the "cartwheel" of Howard and Moore, 1970). It is suggested in some images that the material of the annular granules is in extensive continuity with the projecting tips, thus constituting one whole "cuff" around the pore wall (André and Rouiller, 1956; Watson, 1959; Stevens and Swift, 1966; Stevens and André, 1969; Abelson and Smith, 1970; Franke, 1970a; compare also the model drawings of Gall, 1964; Franke, 1966a; Vivier, 1967; Franke and Scheer, 1970a; Radouco-Thomas et al., 1971; Wunderlich 
and Speth, 1972). However, there is clear demonstration in other nuclei that this is at least not the regular case: here the annular and the internal granules are separated by an electron-transparent gap (e.g., Figs. 16, 21a, b, d-f; see also Daniels et al., 1969; Franke and Scheer, 1970a).

The pore center is frequently, though not always, occupied by a distinct electron-dense particle, the "central granule" (Figs. 4-6, 16-18, 21a and b, 24a-c, 28, and 30) or "central rod" (see Franke, 1970a). Such central dense elements have been demonstrated with all electron microscopic techniques (thin section: Pollister et al., 1954; Afzelius, 1955; shadow cast: Gall, 1954; positive staining: Merriam, 1962; negative staining: Franke, 1966a, b; freeze-etching: Northcote and Lewis, 1968; Roberts and Northcote, 1970; Scheer, 1970a). The diameter of such central elements varies widely. In some nuclei they are relatively uniform in size, whereas in others they vary from pore to pore in a range from ca. 350 to $25 \AA$, the limit of confident identification in current electron microscope preparations of biological material (Figs. 17, 18, 24a-c). Similarly, the three dimensional form and the position of the central element can be greatly variable. It can be located within the very pore center, or lie more eccentrically toward the cytoplasm or the nucleoplasm (Figs. $21 \mathrm{a}$ and $\mathrm{b}, 24 \mathrm{c})$. It can appear as a compact sphere, or as a thin threadlike rod (Figs. 16, 24c, 25), and, just as the granules of the inner annulus, it is connected in most nuclear pore complexes with fibrils which extend far into the nucleoplasm. Such intranuclear fibrillar extensions are therefore often observed as "central elements" within the pore-corresponding channels through the peripheral chromatin (Figs. 20b and c; Franke, 1970a). In various nuclear types the central dense element is in material continuity with, and appears to be a part of, distinct aggregates in a way suggesting an intrapore stage in their nucleocytoplasmic transfer (see below).

In many pore complexes one recognizes various forms of ca. $25-50 \AA$ thick fibrils which are sometimes the only structures identifiable within the pore interior (Figs. 17a, and c, $18 \mathrm{f}$ and $\mathrm{g}$ ). These filaments, which can be set with small granules at variable intervals, are either arranged radially between the central granule and the circumference (or the annular granules, respectively) into a "spoke pattern" (Figs. 17a and d; Vivier, 1967; Yoo and Bayley, 1967; Daniels et al., 1969; Franke, 1970a; Howard and Moore, 1970; Roberts and Northcote, 1970; Hanzely and Olah, 1972), or integrated into a conspicuous "inner ring" (Figs. 18b, c, and e; Yoo and Bayley, 1967; Wunderlich and Franke, 1968; Comes and Franke, 1970; Franke, 1970a; Franke and Scheer, 1970a; Wunderlich and Speth, 1972); occasionally they span the whole pore lumen (Figs. $17 \mathrm{c}, 18 \mathrm{f}$ and $\mathrm{g}$ ). The fibrillar aspects generally appear more prevalent under destabilizing conditions such as the absence of divalent cations 

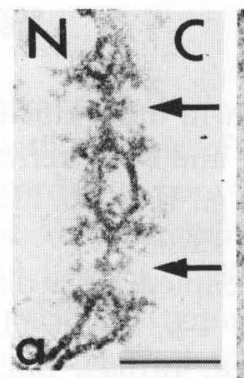

Ni $\bar{c}$

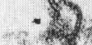

2. 20

int

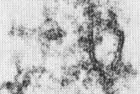

त.s. 3

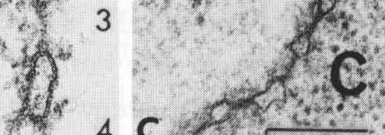

$14 \%$

2yod

$i_{2}$

s.t. 5

$x^{2}+2 y$

1. $x$

(x) 6

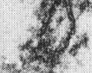

$y_{0}>d$ d

. $x^{2}+8$ का

$x=\frac{1}{2}$

ix 18

$x^{x}=9$

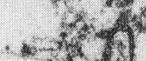

a

b. 10

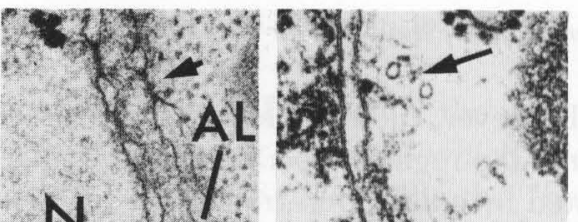

$N+1 ;$

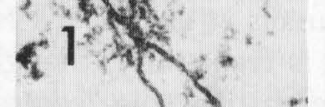

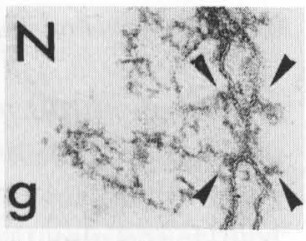

1.

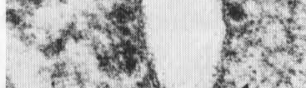

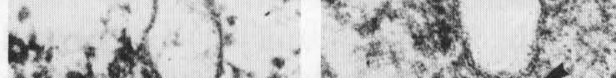

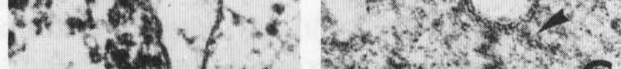

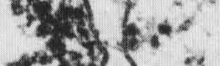
$+2 x+\infty$

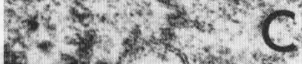
$x^{+}+y^{2}+x \rightarrow$ 2. hit PC $N$ wका ? $+x, 7$ $\therefore e^{72}$
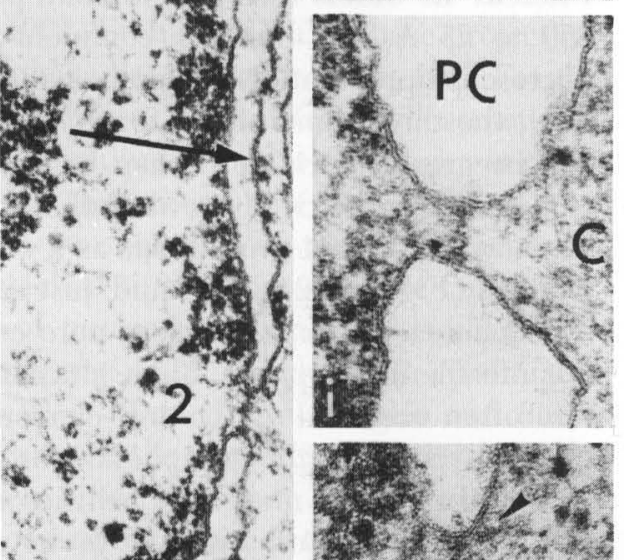

$\therefore 0^{*}+\mathrm{tg}$
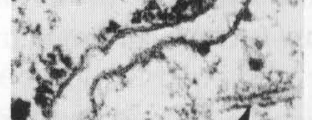

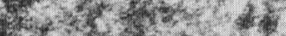

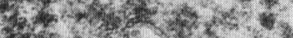

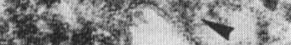
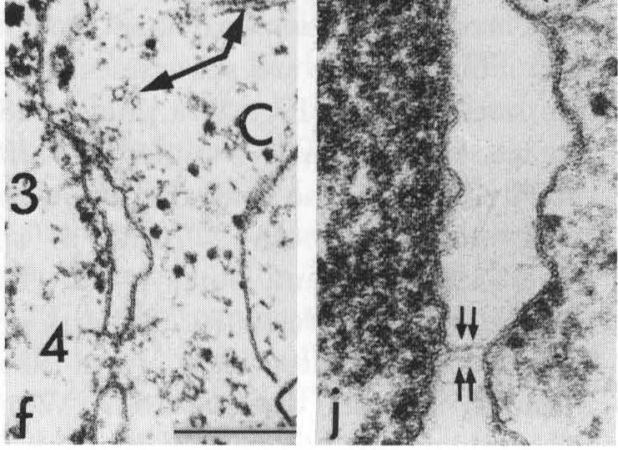

(21) 
or extreme ionic strength (Figs. 4-6; compare Mentré, 1969; Franke and Scheer, 1970a; Franke et al., 1970a; Hanzely and Olah, 1972). It is likely that both the specific preparation method and the specific nuclear type have an influence on the more fibrillar or more compact appearance of the internal pore complex constituents (Franke, 1970a).

\section{Amorphous Material of the Pore Complex}

It has been suggested from the appearance of some indistinct ("cloudy") electron-opaque material surrounding the pore complex com-

Fig. 21 Variations and structural details of nuclear pore complexes in transverse sections. ( $a$ and b) The variability of form, size, and position of the central granule in a nuclear envelope isolated from a maturing Xenopus laevis oocyte. The pore complexes are denoted by arrows in a and by numbers in $b$. The central elements can vary in diameter from ca. $40 \AA$ up to $350 \AA$ (some larger ones are seen in $b$ in the pores designated as Nos. 9 and 10) and sometimes reveal a "light core" (e.g., in the pore denoted by the lower arrow in a). Central elements can be located in the equatorial plane of the pore (e.g., a, and the pores with Nos. 9 and 10 in b) or are displaced from the pore center in nucleoplasmic $(\mathrm{N}$; b, pores with Nos, 1 and 8 ) or cytoplasmic ( $\mathrm{C}$; pore No. 7 ) direction. Sometimes a pore complex containing a central element is associated with another granule which lies on the nucleoplasmic side and is connected to the pore complex by nucleoplasmic fibrils (e.g., at pore No. 4). (c) Annulate lamellae (AL) pore complexes (arrows) in a Xenopus laevis lampbrush stage oocyte showing that interspaces between the nuclear envelope and perinuclear AL can constitute "zones of exclusion" for cytoplasmic organelles and ribosomes. These interspaces contain only fine fibrillar aggregates. The structural differences between the annular granules of the pore complex and the cytoplasmic ribosomes are illustrated in d (nuclear envelope isolated from maturing Xenopus laevis oocyte), e (isolated rat hepatocyte nuclear envelope fragment, no high salt treatment), f (early erythroblast from hen bone marrow), and $h_{-j}$ (maturing hen erythroblasts). Annular granules (denoted by the small arrows in $\mathrm{d}$ and $\mathrm{e}$ ) are larger, less stained, and not so sharply outlined than the ribosomes on the outer nuclear membrane (one is indicated by the upper arrow in d). Moreover, they sometimes reveal a fibrillar substructure aspect (e.g., in the pores with Nos. 2-4 in $\mathrm{f}$ ). This fibril-coil aspect is more pronounced in certain isolation conditions ( $\mathrm{g}$ presents a pore complex of an isolated nuclear envelope from a Xenopus laevis oocyte; annular components are denoted by arrowheads) and in specific cells types such as in the avian erythroblasts (shown in $h-j$ ). In such cells distinct annular particles are sometimes totally unidentified (i), although the equatorial pore material is clearly visible. Note also the inflations of the perinuclear cisterna ( $P C$, in $h-j)$ which seem to be counteracted not only by the pore walls but also by thin intracisternal threads connecting inner and outer nuclear membrane (the small arrows in the bottom part of $\mathrm{j}$ ). Note also the juxtanuclear microtubules (small arrows in f) and the thread connection between the outer membrane of a mitochondrion and the adjacent nuclear envelope (long arrow in $\mathrm{f}$ ). ( $\mathrm{a}, \mathrm{h}-\mathrm{j}, \times 100,000$, bar in a indicates $0.1 \mu \mathrm{m} ; \mathrm{b}, \times 80,000$, bar indicates $0.1 \mu \mathrm{m}$; c, 50,000, bar indicates $0.2 \mu \mathrm{m}$; $\mathrm{d}, \times 120,000$; e, $\times 150,000 ; \mathrm{f}, \times 64,000$, bar indicates $0.2 \mu \mathrm{m} ; \mathrm{g}, \times 90,000)$. 
ponents that, in addition to the distinct granules and fibrils, amorphous (with respect to the limits of resolution of current electron microscopic preparation techniques) material is also present within the annulus and the pore interior (Franke, 1966b, 1967b, 1970a; Franke and Scheer, 1970a).

\section{E. Pore Complex Models}

Since the first interpretation of the annulus-membrane relationship by Afzelius (1955), pore complex models have been repeatedly presented, with profound differences concerning the presence and the relative arrangements of the individual observed structures. Recently, however, various workers have developed, from the early drawings of Afzelius (1955), Watson (1959), Merriam (1961), and Gall (1964), advanced pore complex models which agree in the view that $(a)$ both rims of the pore proper are associated with eight granular subunits equidistantly spaced within the annulus, $(b)$ eight conical or granular clumps project from the pore wall into the interior, $(c)$ various fibrillar arrangements occur within the lumen, and $(d)$ fibrils extend from the granular component, especially from the inner annular and the central granules (Fig. 22; Franke, 1966a, 1970a; Franke and Scheer, 1970a; Roberts and Northcote, 1970; these models were substantially confirmed by LaCour and Wells, 1972, and Wunderlich and Speth, 1972). These models differ, however, from those of Dawson et al. (1955), Wischnitzer (1958), Nørrevang (1965), Sichel (1966), Vivier (1967), Abelson and

Fig. 22 Earlier (a; from Franke, 1966a) and recent model views of the pore complex: The pore fenestrae are associated with annular granules. Eight annular granules lie on either rim of the pore, regularly spaced and arranged in a radial symmetry. Tips of dense material project conically from the pore wall (the "peripheral granules" sensu Roberts and Northeote, 1970, 1971) and can also be arranged in an eightfold radial symmetry, sometimes in correspondence with that of the annular granules. The pore center is frequently occupied by a dense element of variable shape and size. The massive projecting tips contain fibrils which can be directly visualized in certain cells and preparations. Such internal fibrils can be radially arranged (often connecting the central granule with the pore wall and the annular granules, respectively) and can also constitute a so-called inner ring structure. Fibrillar threads are also frequently seen in continuity with the central and annular granules, especially at the inner (nucleus-oriented one) annulus. While the model of $b$ emphasizes the compact appearance of the nonmembraneous constituents of the pore complex (slightly modified from Franke, 1970a, and Franke and Scheer, 1970a), c presents an alternative view with emphasis on the fibril-coil aspect of the annular and internal pore complex structures (modified from Franke, 1970a). For clarity, only one of the "projecting tips" has been included as a fibrillar aggregate in the drawing of $c$. 

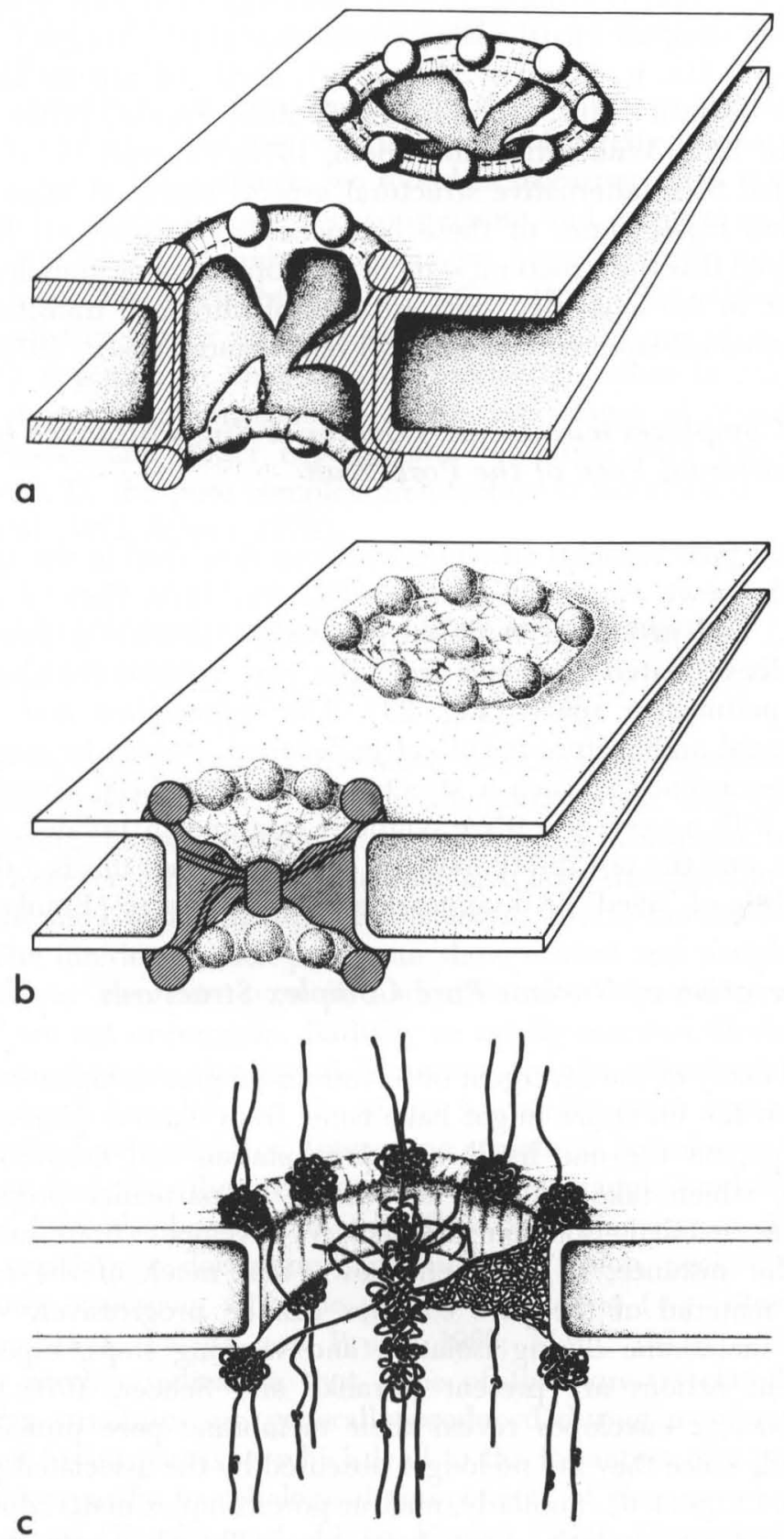

(22) 
Smith (1970), and Herrlinger (1970). Although most micrographs suggest a rather compact (particulate) appearance of pore complex granules (Figs. 16-19, 21a, b, d, and e, 22a and b), fibril-coil aspects can sometimes also be seen (Figs. 21c, f-h Kessel, 1969; Franke, 1970a; LaCour and Wells, 1972; Wunderlich and Speth, 1972; Fabergé, 1973). It may well be that these alternative structural aspects represent more densely packed and looser states of these components. Moreover, it has been hypothesized that the structural state of the pore complex granules in vivo is dynamic in the sense that they are sites of fibril-coil transitions, perhaps of ribonucleoprotein material (Franke and Scheer, 1970a).

\section{F. Pore Complexes with Dense Equatorial Rings Attached to the Intracisternal Face of the Pore Wall}

A special pore complex structure has been described in the spermatocytes of the newt, Pleurodeles waltlii (Picheral, 1970; Picheral and Folliot, 1971) and in late erythroblasts (Fawcett, 1966b). A thin ring of electron-dense material extends from the pore equator for about $80 \AA$ into the perinuclear space (Fig. 28). The organization and meaning of this special architecture is not yet understood. It must be emphasized that the occurrence of such a structure has so far been reported only in nuclei with a very low RNA synthesis rate and in isolated envelope fragments, and the possibility must be considered that this is a deviation characteristic of "aged" or disappearing pore complexes (Franke, 1974).

\section{G. Preservation of Nuclear Pore Complex Structures}

A good many of the divergent observations on pore complex structures reported in the literature might have come from various degrees of destabilization, on the one hand, and precipitation and dehydration, on the other, which take place in the course of particular preparations. Concerning manual isolation of nuclear envelopes from amphibian oocytes, for instance, it has been shown that much of the nonmembraneous material of the pore complex can be progressively removed from the membrane during isolation and washing steps, especially if no divalent cations are present (Franke and Scheer, 1970a). Thus, washed nuclear envelopes reveal their membrane pore profiles much more clearly since they are no longer obscured by the associated particles and fibrils (Figs. 4-6). Similarly, nuclear pore complex material is diminished during mass isolation procedures, especially when extraction with high salt concentrations has been applied (Mentré, 1969; Franke et al., 
1970a). On the other hand, it is important to note that to some extent pore-associated, nonmembraneous structures are retained after such high salt extractions at all.

In addition, there are some indications that structures associated with nuclear pores might disappear in the course of certain nuclear differentiation processes. For instance, we found in maturing avian erythrocytes that central granules become less conspicuous and might even be undiscernible, and distinct annular granules are likewise hardly found (Fig. $2 \mathrm{lh}$ and i; see also Kartenbeck et al., 1971). It must be added, though, that this disappearance is not a necessary consequence of a near (or complete) cessation in nuclear RNA production, since in other nuclei inactive in transcription, such as in the late stages of oogenesis and spermiogenesis and under experimental inhibition with the antibiotic actinomycin D, the pore complex architecture is maintained (Fig. 18d; Eckert et al., 1972; Scheer, 1973).

\section{H. Pore Complexes Compared to Other Membraneous Pore Formations}

Pore complexes of exactly the same substructure organization as in the nuclear envelope occur only in the intranuclear and cytoplasmic $\mathrm{AL}$, including the occasional pore complexes in ER cisternae mentioned earlier. However, other pore formations have been described in cisternae, e.g., of ER and Golgi apparatus (Franke et al., 1971b; Franke and Scheer, 1972). The interior of such pores can show central and peripheral nonmembraneous elements as well, and structures resembling a "central granule" are not uncommon. Radially or axially oriented fibrils attached to the pore rims are also found. However, the size distribution of such pores is much broader and the shape is usually much more irregular than in true pore complexes. Interesting structural homologies with pore complexes, including the presence of a "central granule" and of radiating filaments, have also been noted in the fenestrae (diameter ca. 600-800 $\AA$ ) of the capillary endothelium, and in the "fusion necks" of vesicle membranes coalescing with the plasma membrane (e.g. Rhodin, 1962; Friederici, 1968; Palade and Bruns, 1968; Maul, 1971b). This might serve as another indication that some of the pore-associated globular or fibrillar structures are generally produced during membrane breakdown and fusion processes which lead to the formation of a pore, rather than being specific morphological components of the pore complex described above (Franke et al., 1971b; Franke, 1974). Characteristic annular structures do not occur in such pore formations and might therefore 
represent the best "marker" structure in distinguishing a true pore complex of the nuclear envelope and AL.

\section{Pore Complex Formation and Disappearance}

Ideas and observations relevant to de novo pore complex formation have been presented by various authors (Schjeide, 1970; Franke et al., 1971b; Gulyas, 1971a; Maul et al., 1971; Franke and Scheer, 1972; Franke et al., 1972a; Scheer and Franke, 1972) but the situation is far from being clarified. One should emphasize, however, that nuclear pore complexes can be formed de novo within an existing closed envelope at various rates. Net pore formation rates range from less than 1 pore/min to 25 pores/min in HeLa cells (Maul et al., 1972) and even to 480 pores/min in the lampbrush stage Xenopus laevis oocyte (Scheer, 1973). Pores also appear in the isolated nuclear envelope fragments associated with chromosome surfaces in mitotic anaphase-telophase stages during nuclear envelope reconstitution (see Section XII). It is also obvious that pore complexes disappear, e.g., during late erythropoiesis and during sperm maturation, often selectively in only one nuclear envelope region (see Sections VII, M and N, and IX). Any hypothesis for how pore complexes are formed in the nuclear envelope should also require an explanation of pore complexes in AL. The suggestion by Maul et al. (1972) that pore formation is correlated to chromosome replication is certainly not generally true, as demonstrated by the enormous increase in nuclear pore complexes in the diplotene stage of oogenesis in amphibia (Merriam, 1962; Franke and Scheer, 1970b; Scheer, 1973) and the reappearance of pores in late phases of some sperm maturations (Stanley, 1971b), at which stages no significant DNA synthesis takes place. Moreover, a correlation with replication is also excluded for the pore complex formations which occur during nuclear envelope reconstitutions in various nuclear divisions and in the pronuclear formations after fertilization (see Section XII).

\section{J. Morphological Relationships and Cytochemical Reactions of the Nuclear Pore Complex Constituents}

It has been suggested by a series of authors that chromosomal fibers are attached to the inner annulus (Claude, 1964; DuPraw, 1965, 1968, 1970; Comings and Okada, 1970a; Lampert, 1971; Maul, 1971a; Engelhardt and Pusa, 1972; LaCour and Wells, 1972; Sorsa, 1972). From equating observed fibrils with chromatin deoxyribonucleoproteins (DNP), views have arisen that $(a)$ the inner annular granules (and perhaps the central 
granule, too) might represent the attachment sites of chromosomal DNP, (b) inner annulus-attached fibrils generally represent chromatin DNP strands, and $(c)$ the nonmembraneous moieties of the pore complex might in general contain DNP (Comings, 1968; DuPraw, 1968; Comings and Okada, 1970a; Lampert, 1971; Engelhardt and Pusa, 1972; Sorsa, 1972). The concept of the DNP nature of inner annulus-attached fibrils has been based on demonstrations of the association of electron-dense (or densely stainable) fibers, in material continuity with chromatin, with the inner annulus during interphase and various mitotic and meiotic stages (Comings and Okada, 1970a, b; Lampert, 1971), and on the notion of DuPraw (1965) that such fibrils were digested after treatment with trypsin followed by pancreatic deoxyribonuclease. However, neither do the fibrils presented unequivocally show attachment to individual annuli nor does this demonstrated DNase sensitivity of the bulk of the nuclear chromatin fibrils allow a conclusion as to the specific DNA content of the putative annulus-attached fibrils. As to the compounds contained in the nonmembraneous pore complex material, there are a number of points one must consider. (i) Both inner and outer annuli, as well as the central elements, frequently show a variety of direct morphological continuities with known ribonucleoprotein (RNP) structures such as the cytoplasmic polyribosomes (Figs. 18a, 30a-d; see also Section VII, B), the nucleolar periphery (pars granulosa; Figs. 23a-d, 24d-e Horstmann and Knoop, 1957; Drawert and Mix, 1961; Werz, 1964; Lane, 1967; Kessel, 1968b, c; Kessel and Bearns, 1968, 1969; Scharrer and Wurzelmann, 1969a; Ulrich, 1969; Franke and Falk, 1970; Franke and Scheer, 1970b; Franke et al., 1974; for further references see Franke, 1974), the granular aggregates detached from the nucleolar periphery or from the Balbiani rings of chironomid salivary gland chromosomes (e.g., Figs. 23e-g, 25a-e, 26; Beermann, 1964; Swift, 1965; Stevens and Swift, 1966; Takamoto, 1966; Lane, 1967; Scharrer and Wurzelmann, 1969b; Franke and Scheer, 1970b; Cave and Allen, 1971; Eddy and Ito, 1971; Lasek et al., 1972), and, via very thin filaments, with distinct RNP granules including the perichromatin granules (Watson, 1962; Monneron and Bernhard, 1969; Franke, 1970a; Petrov and Bernhard, 1971). This is diagrammatically summarized in Fig. 27. (ii) Distinct and defined regions of chromatin attachment sites to the inner nuclear membrane are found between the pores (see Section V). (iii) Pore complexes in chromatin-free regions of the nuclear envelope show the normal architecture. Such regions are identified in meiotic prophase stages, in the "redundant" (sometimes even delaminated) and folded-back sections of the nuclear envelope observed during sperm development (Fig. 28; e.g., Horstmann, 1961; Brökelmann, 1963; 

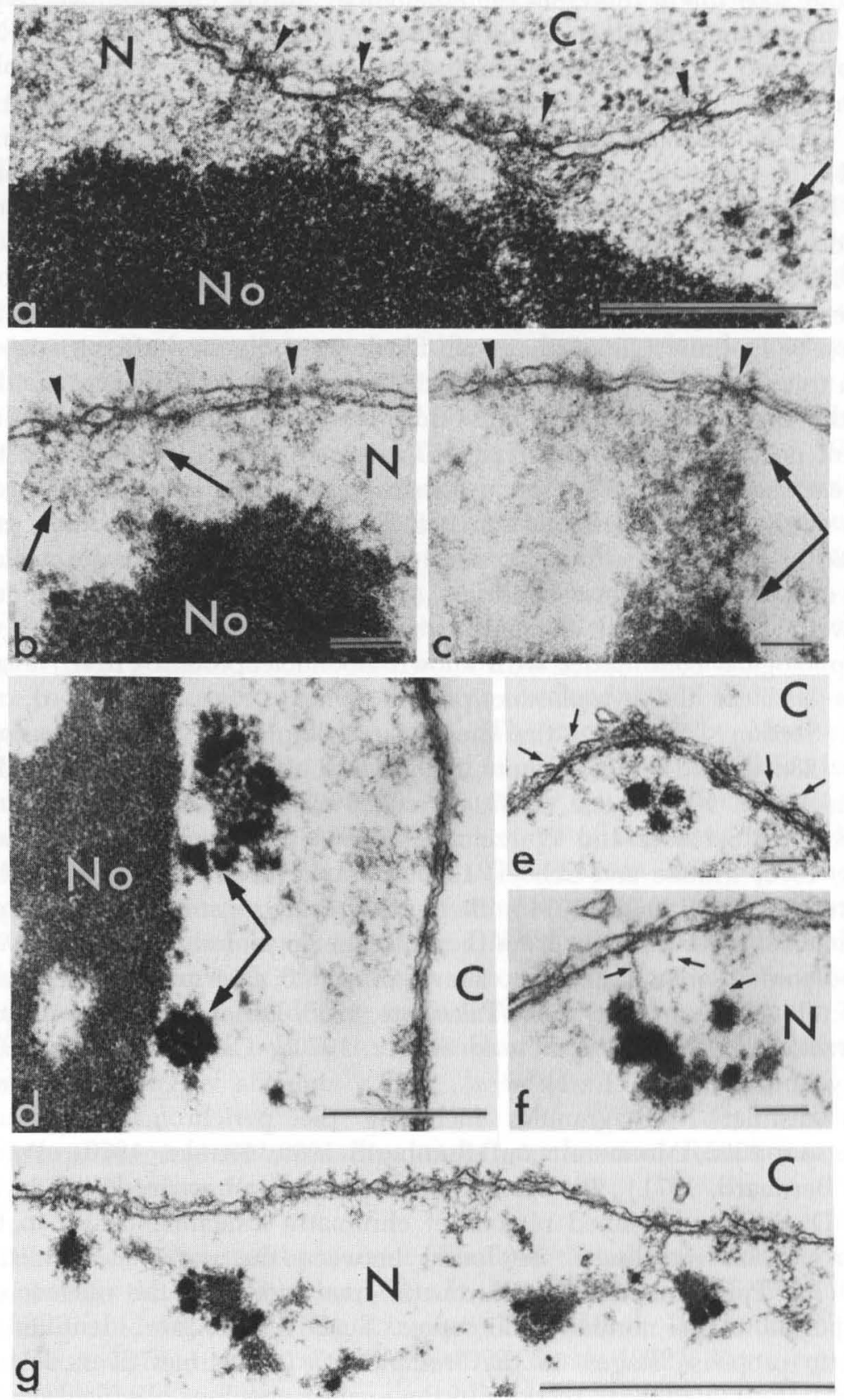
Werner, 1966; Franklin, 1968; Plattner, 1971; Rattner and Brinkley, 1971; Stanley, 1971a; Wooding and O'Donnell, 1971), in the mid-portion of the mother nuclear envelope remaining after reconstitution of the nuclear envelope of daughter nuclei in the nuclear divisions of various algae, fungi, and ciliates (nuclear envelope remainder, separation spindles; Marchant and Pickett-Heaps, 1970; Ott and Brown, 1973; see also Section XII; for an example of absence of pores in a special separation spindle see Tucker, 1967), in the polar parts of the metaphase micronucleus in some ciliates (Tucker, 1967), and in fragments of perinuclear cisterna discernible freely in the cytoplasm after nuclear envelope breakdown ( see Section XII). Likewise, AL pore complexes also show annulus-attached fibrils, central granules, and all the other structural details, but are very unlikely to contain DNP. (iv) Cytochemical studies using Bernhard's chelating agent method (1969) showed that pore complex structures retain uranyl stain similar to RNP structures and are different from DNP (Figs. 29 and 30; Franke and Falk, 1970; Esponda and Stockert, 1972). The pore complex material is diminished after treatment with cold perchloric acid (Monneron and Bernhard, 1969). Results obtained with ribonuclease are more conflicting. Mentré (1969) reported a specific digestibility of the pore complex granules with this enzyme, whereas other authors had contrary results (Merriam, 1961; Beaulaton, 1968; Koshiba et al., 1970). Figure 31 shows the result of a ribonuclease treatment of isolated nuclear envelopes from Xenopus laevis oocytes: in a positively stained spread preparation the electron-dense pore complex material, including the central granules, can still be identified. On

Fig. 23 Morphological associations of nuclear pore complexes with nucleolar (No) and nucleolus-derived structures in amphibian oocytes (lampbrush stage in Triturus alpestris, a-f, and in Xenous laevis, g). Coarse electron-dense emanations from the nucleolar cortex protrude toward the nuclear pore complexes (denoted by arrowheads in $\mathrm{a}-\mathrm{c}$ ). Such pore complex-nucleolus-connecting material is frequently resolved as consisting of granulofibrillar aggregates (arrows in $\mathrm{b}$ and $\mathrm{c}$ ). The fibrils often appear to terminate at the inner annulus and/or the central granules as is suggested for the fibrils (indicated by the arrows in b). Distinct aggregates of granules which appear to detach from the nucleolar cortex in these oocytes (the "streams of granules" described by Lane, 1967) are also connected with the pore complexes, in particular with the inner annuli, via thin fibrils ( $d-g$; the arrows in a and $d$ point to two such aggregates still associated with the nucleolar cortex; the small arrows in e point to outer annuli of pore complexes, whereas those in $f$ denote fibril connections of nucleolar-derived aggregate granules with the inner annuli). Such nucleolus-derived granules ( $g$ ) are occasionally retained at isolated nuclear envelopes, thus illustrating the relative stability of the fibrillar connections. $N$, nucleoplasmic side; $C$, cytoplasmic side $(a, \times 53,000 ; b, \times 86,000 ; c, \times 60,000$; $\mathrm{d}_{\text {, }} \times 40,000 ; \mathrm{e}, \times 56,000 ; \mathrm{f}, \times 64,000 ; \mathrm{g}, \times 36,000$; the bars in $\mathrm{b}, \mathrm{c}, \mathrm{e}$, and $\mathrm{f}$ indicate $0.1 \mu \mathrm{m}$, those in a and $\mathrm{d}$ represent $0.5 \mu \mathrm{m}$, the bar in $\mathrm{g}$ indicates $1 \mu \mathrm{m}$ ). 

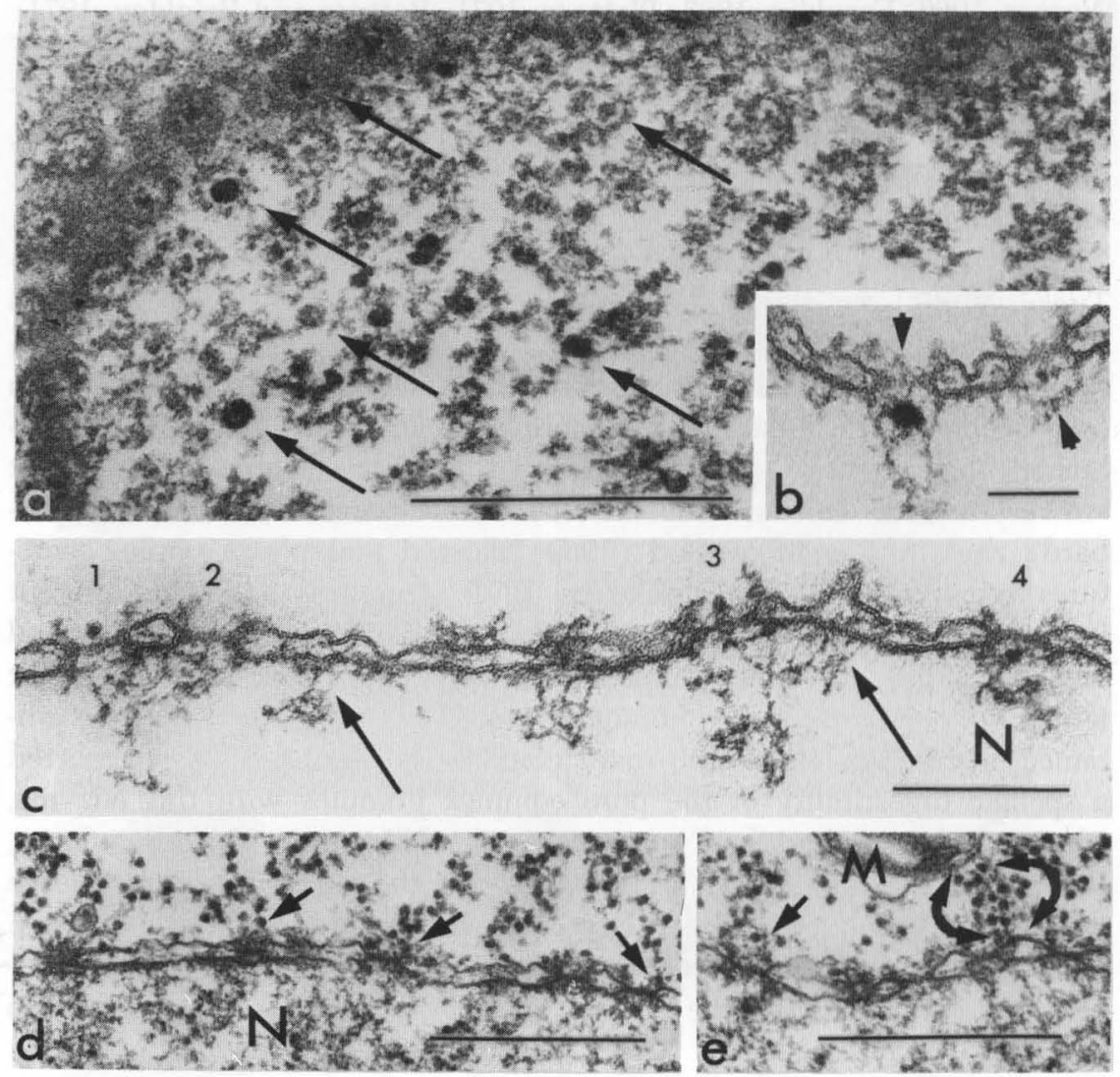

(24)

Fig. 24 Further examples of associations of ribonucleoproteinaceous (RNP) structures with nuclear pore complexes in the primary nucleus of Acetabularia mediterranea (a) and in amphibian oocytes (from Xenopus laevis, b-e). Large nuclear globules (up to $500 \AA$ in diameter, denoted by the arrows in a and the left arrow in b) appear to enter the pore complexes and can be identified as "central pore elements." However, they do not pass the pore as a whole and are not seen on the cytoplasmic side. The two pore complexes in b (arrows) also demonstrate the size variability of the various forms of centrally located granules. In c, note that nucleoplasmic RNP fibrils, in addition to their attachment to the pore complexes, occasionally are also seen at interporous regions of the inner nuclear membrane (arrows). Note also the different positions of the central elements with respect to the pore complex axis (pores numbered as 1,3 , and 4 ) whereas pore No. 2 does not show a central element at all. In $\mathrm{d}$ and $\mathrm{e}$ are seen the outer annulus association of cytoplasmic polyribosome formations (arrows) which can even connect the nuclear pore complexes with surfaces of $\mathrm{ER}$ and mitochondria ( $\mathrm{M}$; indicated at the curved arrows in e) $(\mathrm{a}, \times 66,000$; b, c, $\times 90,000$; $\mathrm{d}, \times 44,000$; $\mathrm{e}, \times 50,000$; the bar in $\mathrm{b}$ indicates $0.1 \mu \mathrm{m}$, that in $\mathrm{c} 0.2 \mu \mathrm{m}$, those in a, d, and e $0.5 \mu \mathrm{m} ; \mathrm{N}$, nucleoplasmic side). 
the other hand, a convincing illustration of ribonuclease-induced removal of material stainable with indium trichloride, a nucleic acid stain, from nuclear pore complexes of oocytes from the frog, Rana, is contained in the study of Eddy and Ito (1971; their Fig. 15; see, also Conway, 1971). RNase sensitivity was also specifically reported for the various central element structures which can be found in pores (Stevens and Swift, 1966; Cole, 1969; Mentré, 1969; see, however, Koshiba et al., 1970). Results with DNase have been negative (Mentré, 1969; compare Beaulaton, 1968; Koshiba et al., 1970). All these authors agree that proteases in general are capable of removing the pore complex structures to a considerable extent (see also Abelson and Smith, 1970). That DNA is not present within the pore complexes in important quantities has also been demonstrated by negative results with binding of tritiated actinomycin $D$, a method that is sensitive enough to detect even the small amount of DNA within the extrachromosomal nucleoli in the amphibian oocytes (Scheer, 1972). (v) When manually isolated nuclear envelopes from Xenopus laevis oocytes were biochemically analyzed, no DNA but a considerable amount of RNA was detected (Scheer, 1972). In gel electrophoresis, this RNA exhibits a distinct pattern (Fig. 32), compared with RNA from total nuclei, suggesting a special enrichment of rRNA-processing intermediates in the molecular weight range from 2.0 to 1.6 million daltons. Since such preparations do not contain structures other than nuclear membranes and the fibrils and granules associated with the pore complexes (see Figs. 3 and 4), it was concluded that most of this RNA recovered with isolated nuclear envelopes is contained in the pore complex structures, in the form of RNP. By combination of these determin ?tions with morphometric data obtained from the same fraction, the RNA content per average pore complex $\left(4 \times 10^{-5} \mathrm{pg}\right)$ and the RNA package density within these nonmembraneous structures of the pore complexes was also calculated (Fig. 33). The latter is between the values for the nucleolus and cytoplasmic ribosomes (Scheer, 1972). These data would fit in with electron microscopic determinations of total pore complex dry mass by DuPraw and Bahr (1969).

Taken together, there appears to be only weak support for a DNP nature of the inner annulus-attached fibrils and the other pore complex constituents, but there are many more indications that they contain RNP. Some of the nonmembraneous pore complex structures might also contain lipoprotein material of a membraneous origin which has not rearranged into the membrane leaflet structure after pore formation (Franke et al., 1971b). However, except for one notion by Mentré (1969) on a differential sensitivity of annular and central granules in high salt extractions and ribonuclease treatments (see, however, Franke 


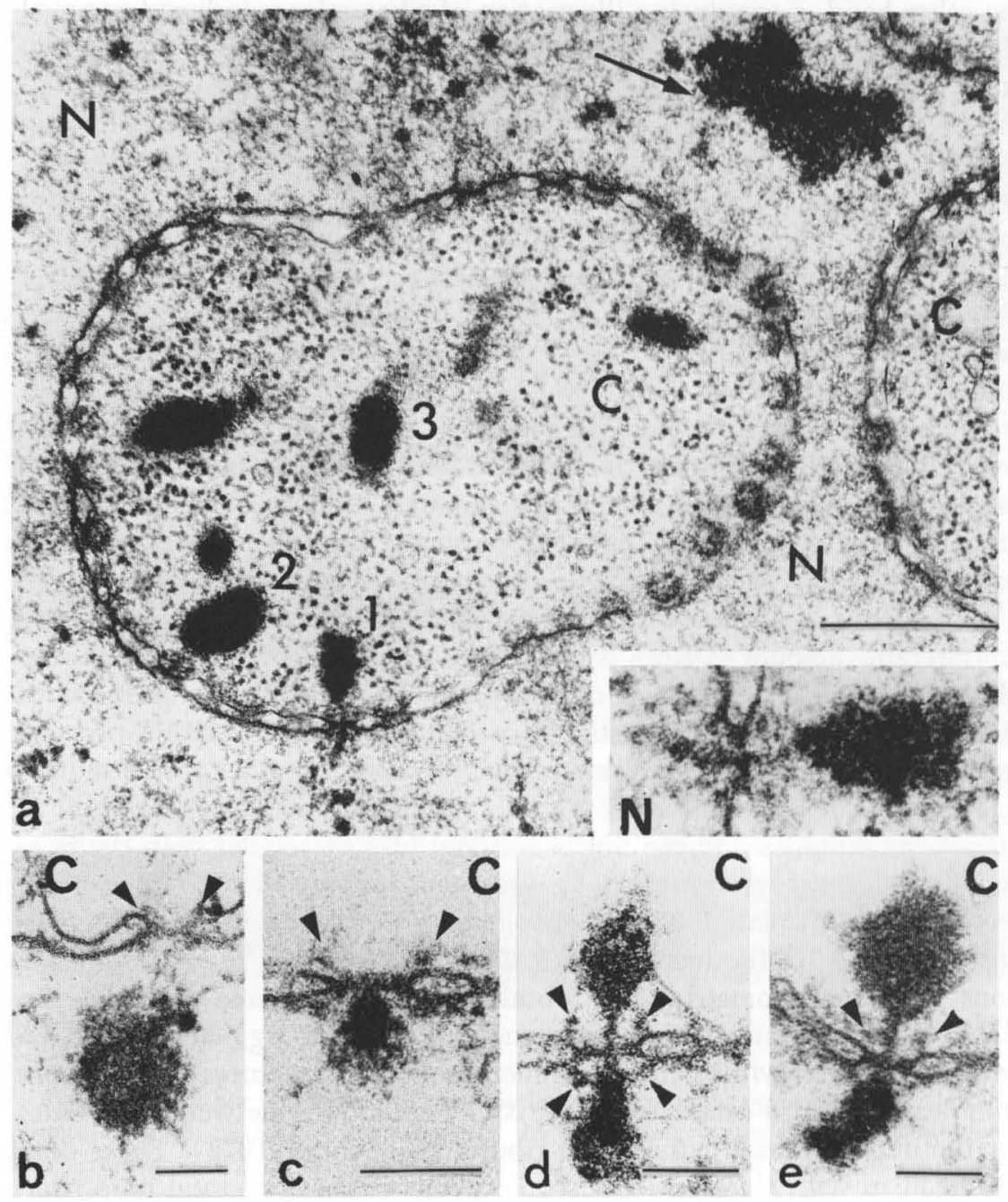

(25)

Fig. 25 Electron micrographs of the nuclear periphery in lampbrush stage oocytes of Triturus alpestris (a, c-e) and Xenopus laevis (Fig. b) demonstrating the nucleocytoplasmic emission of large material clumps which probably originate in the nucleolar periphery and might contain RNP. Such aggregates are seen in the nucleus (at the arrow in a), in association with nuclear pore complexes (a-e), and in the perinuclear cytoplasm ( $a$; the Nos. 1-3 indicate the putative time sequence of the migration through, and the detachment from, the nuclear pore complex). The inset in a shows the pore association of the aggregate No. 1 at higher magnification. In $\mathrm{b}-\mathrm{e}$ is seen the possible sequence of events in the course of the penetration of the 

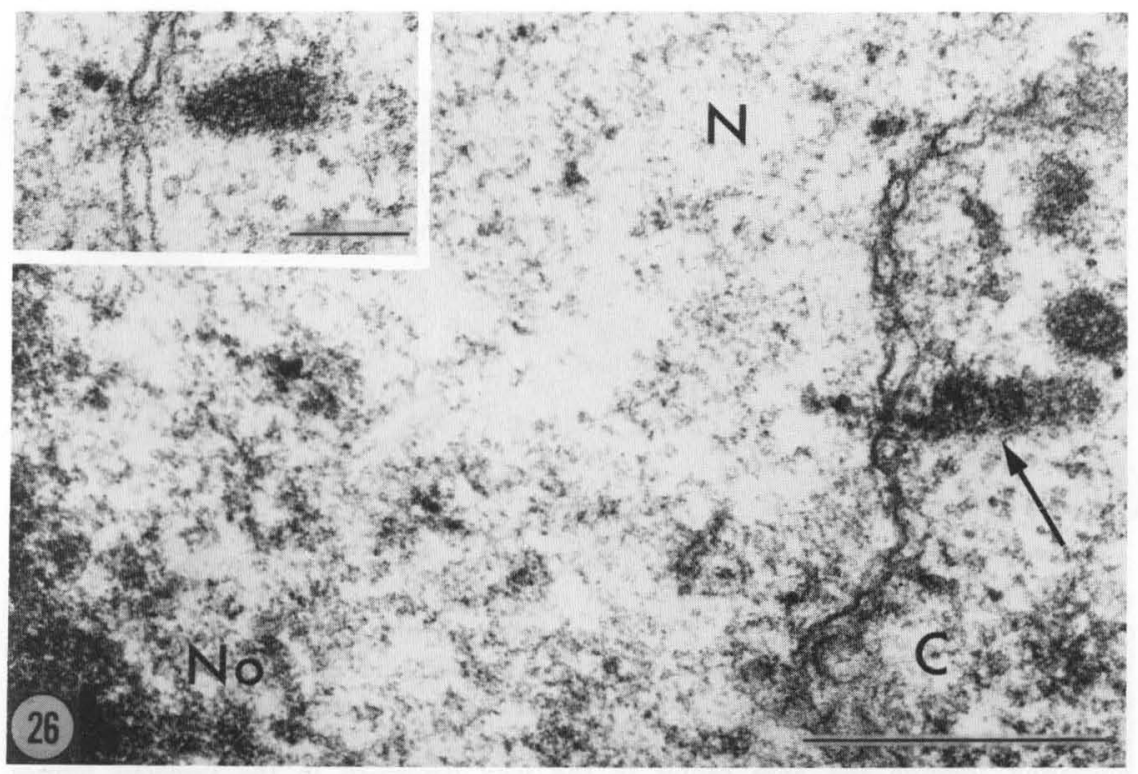

Fig. 26 Previtellogenic stage of a Xenopus laevis oocyte showing similar emission of aggregates through nuclear pore complexes (arrow; the inset shows an aggregate associated with the cytoplasmic side of a pore) as in the previous figure. This early stage in oogenesis, however, is characterized by a predominance of synthesis and accumulation of low molecular weight RNA (4 S, 5 S; compare Thomas, 1970, Denis and Mairy, 1972) and a scarcity of ribosomes in the cytoplasm, contrary to the later lampbrush stages in which the vast part of RNA produced and nucleocytoplasmically transported is represented by the high molecular weight rRNA (Davidson et al., 1964). N, nucleus; C, cytoplasm; No, nucleolus ( $\times 61,000$, bar indicates 0.5 $\mu \mathrm{m}$; inset, $\times 60,000$, bar indicates $0.2 \mu \mathrm{m}$ ).

pore complexes (some annular granules are denoted by arrowheads): the large globule approaches the pore complex and becomes connected to it by thin filaments (b); it then reaches the pore center (c) and elongates into a 100-150- $\AA$ broad rod; the material passes the pore center in this rodlike form, transitorily assuming a typical dumbbell-shaped configuration (d); then the material rounds into a spheroid particle (e) and is deposited on the cytoplasmic side, for some time still revealing fibrillar connections with the pore complex through which it came (e.g., at the aggregate No. 2 in a). Such aggregates entrapped in the pore complex are retained with isolated nuclear envelopes ( $\mathrm{b}-\mathrm{e}$ ); a shows a grazing section to a highly invaginated nuclear surface. $\mathrm{N}$, nucleoplasmic side; $\mathrm{C}$, cytoplasmic side (a, $\times 39,000$, bar indicates $0.5 \mu \mathrm{m}$; inset, $\times 100,000 ; \mathrm{b}, \times 83,000 ; \mathrm{c}, \times 135,000 ; \mathrm{d}, \times 110,000$; $\mathrm{e}, \times 100,000$, bars in b-e indicate $0.1 \mu \mathrm{m}$ ). 


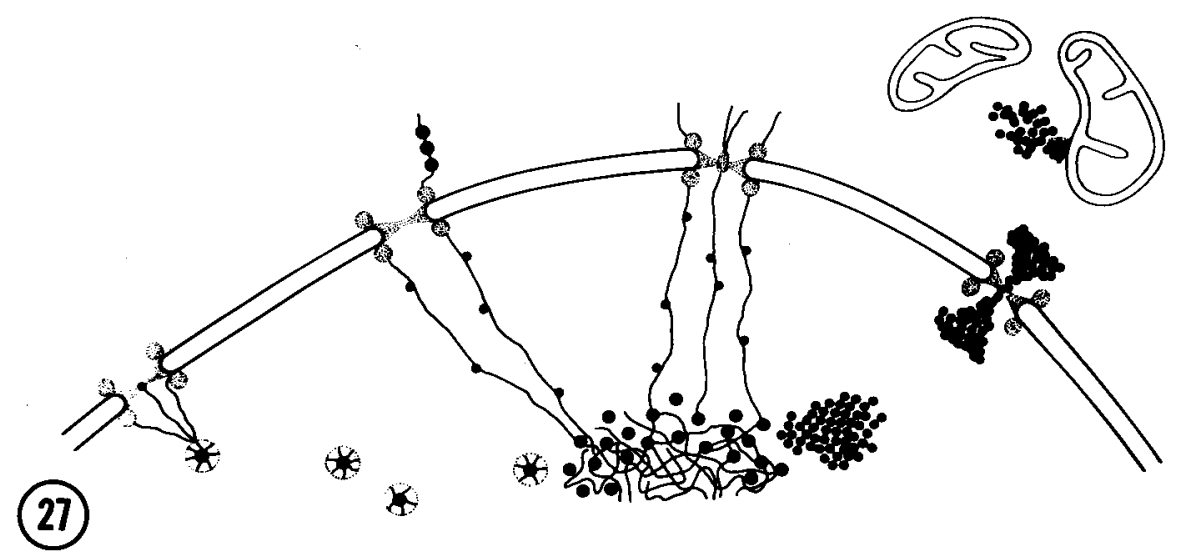

Fig. 27 Diagrammatic summary of the various associations of nuclear pore complexes with structures of a (probably) ribonucleoprotein content. Associations of central and inner annular granules have been reported for perichromatin granules (at the pore in the very left) and for the granulofibrillar bundles originating from the nucleolar cortex (at the two central pores). The outer annulus shows associations with cytoplasmic polyribosomes (at the second pore from the left). Large aggregates migrating through the pore center in the way described in the previous figures (sketched at the pore in the right) are deposited in the juxtanuclear zone, frequently in close association with mitochondrial surfaces.

et al., 1970a), there is so far no experimental indication of differences in the chemical composition of the various pore complex components.

\section{K. Cell Differences in Nuclear Pore Complex Architecture}

Although the arrangement of the pore complex constituents appears to be essentially universal among eukaryotic cells (Franke, 1970a), some variations in the structural aspect are noticeable. As already mentioned, some cells show the annular, peripheral, and central granules as loose, fibrillar aggregates rather than as compact granules, and in some nuclear types annular granules are hardly seen at all (Fig. $21 \mathrm{~h}$ and i). It is not clear, however, whether such apparent variations reflect true cell differences in pore complex structure, or are rather due to the reaction of a specific cell type to the particular preparation method applied. At least the latter possibility is suggested from the importance of the ionic strength and composition and the content of divalent cations in media used for isolation and/or fixation (Franke and Scheer, 1970a; Hanzely and Olah, 1973). Changes in the nuclear pore complex structure have been reported as occurring in the large envelope parts persisting 


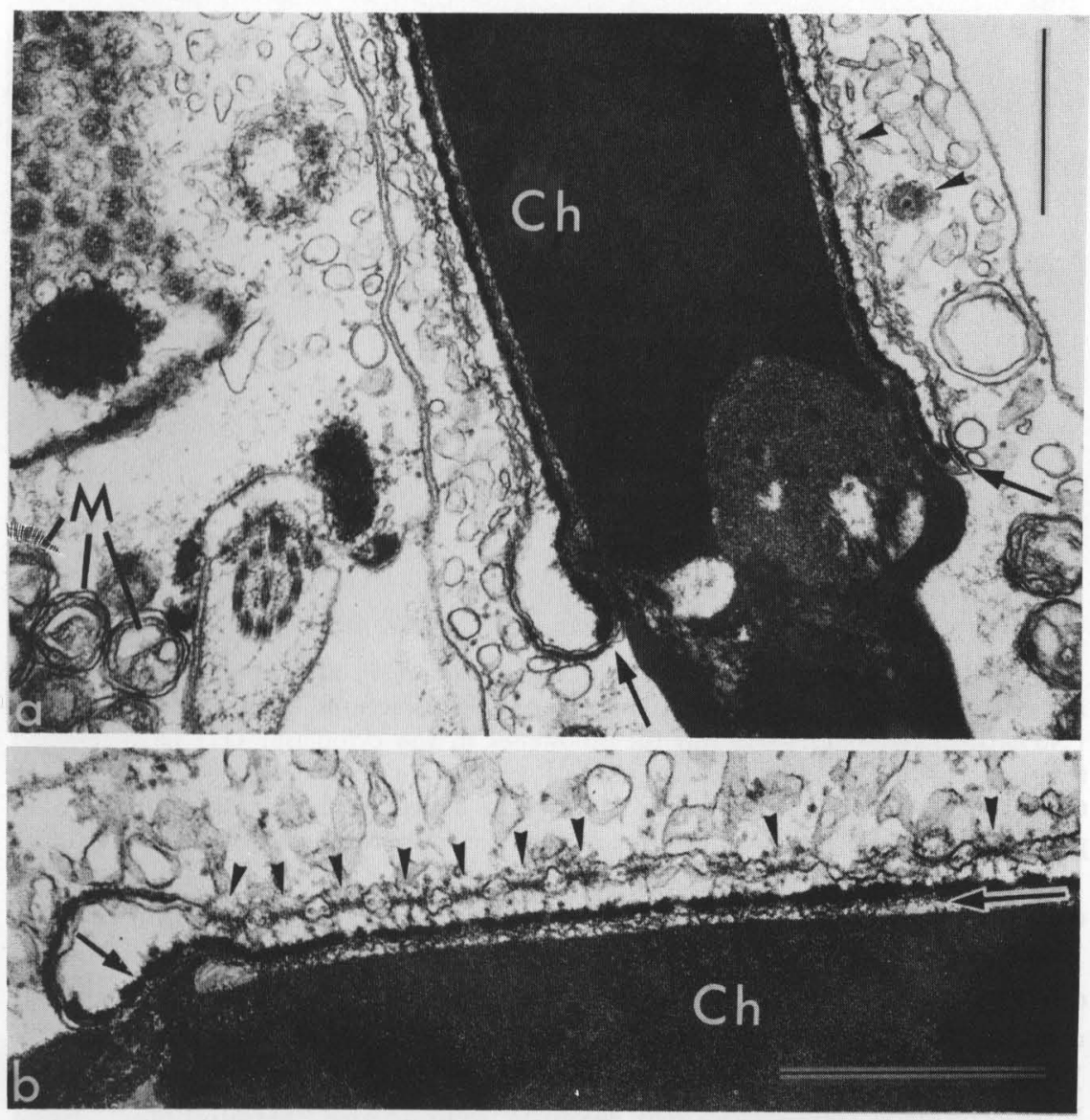

28

Fig. 28 Folding-back of the nuclear envelope in late stage of sperm maturation in the newt, Triturus alpestris, is indicated at the arrows in a. This process surrounds the pericentriolar mass of "chromatoid" material $(\mathrm{Ch})$. Note that the pore complexes of the delaminated nuclear envelope part reveal a normal substructural organization with annular granules and the various internal structures, including the central granule (arrowheads; see also the tangential section in the upper left of a). Note the intracisternal projections from the pore walls in the equatorial plane. The arrow in the right of $b$ points to that part of the nuclear envelope which borders the pericentriolar mass $(\mathrm{Ch})$ and which appears to be characterized by a scarcity, if not absence, of nuclear pore complexes and a very narrow perinuclear space. Note that the inner membrane of this nuclear envelope section is coated with densely stained material (indicated at the arrow in the left of b) which shows thread continuities with the pore complexes of the adjacent nuclear envelope part. $\mathrm{M}$, mitochondria (a, $\times 40,000 ; \mathrm{b}, \times 56,000$, bars indicate $0.5 \mu \mathrm{m}$ ). 


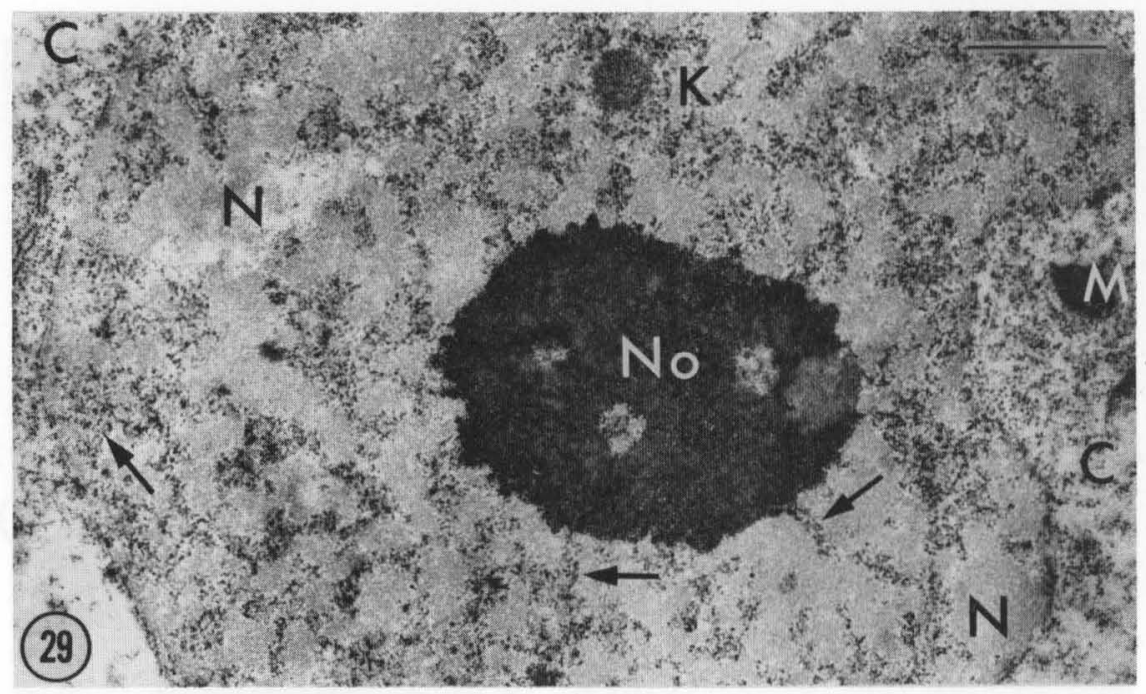

Fig. 29 Appearance of an onion root tip nucleus (N) after the selective staining method of Bernhard (1969). Ribonucleoprotein structures such as the nucleolus (No), the karyosome (K), and the ribosomes in the cytoplasm (C) have retained the stain as well as the fibrillar strands (some are indicated by arrows) which traverse the "bleached" chromatin and which appear to terminate at the nuclear pore complexes. M, mitochondrion ( $\times 14,000$, bar indicates $1 \mu \mathrm{m})$.

during intranuclear mitosis in Chlamydomonas (Johnson and Porter, 1968), and have also been discussed as possible explanations for the different pore complex aspects observed in various physiological and cell cycle stages in macronuclei of Tetrahymena pyriformis (Franke, 1967b; Wunderlich and Franke, 1968; Wunderlich, 1969a). Moor and Mühlethaler (1963; see also Frey-Wyssling and Mühlethaler, 1965; DuPraw, 1970) have observed differences in nuclear pore complex appearance in freeze-etch preparations of yeast and have interpreted this as indication for two alternative functional states of nuclear pores, open and sealed, and that the latter are more frequent in old yeast cells. These authors have also shown patches of open and sealed pores in the same nucleus, and thought that an "active opening and closing of the pores" might be a means of regulating nucleocytoplasmic exchange.

\section{Pore Complex-Connecting Thread Structures}

Fibrillar threads which span the membrane area between adjacent pore complexes have been noted in negatively stained preparations of isolated nuclear envelopes (Franke and Scheer, 1970a; Fabergé, 1973), 
in sections tangential to the nuclear envelope (Fig. 18a; Speth and Wunderlich, 1970), and in freeze-cleave preparations (Scheer, 1970a; Speth and Wunderlich, 1970; Kartenbeck et al., 1971). While with the first two preparation methods such structures could be interpreted as representing collapsed annulus-attached fibrils, the freeze fractures strongly suggest, on the basis of Branton's (1966; Branton and Deamer, 1972) idea that fracture planes generally run in the membrane interior, that the fibrils seen in freeze-etch preparations are material located within the membrane lamella.

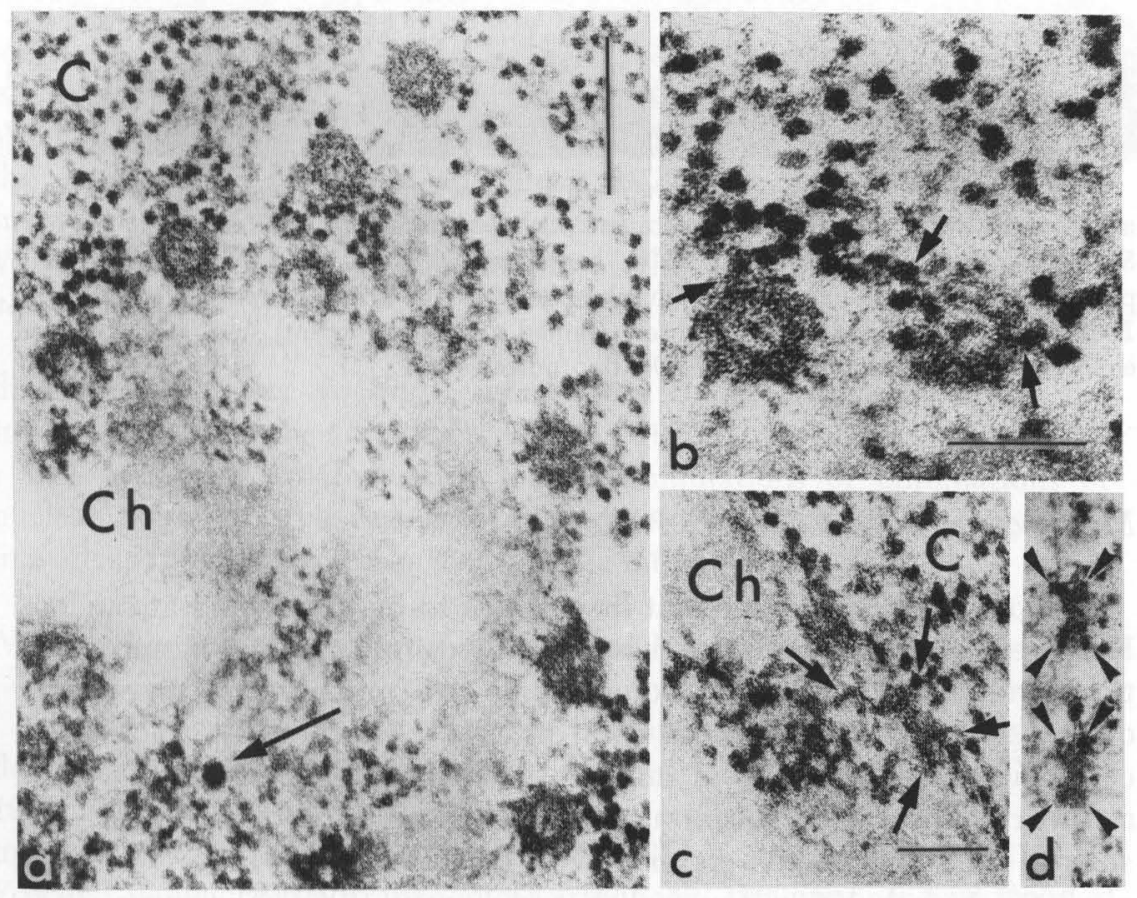

\section{(30)}

Fig. 30 Same preparation as in Fig. 29. The chromatin ( $\mathrm{Ch}$ ) is almost totally bleached but the pore complex substructures including the annuli and the central elements appear stained, although less than the ribosomes in the cytoplasm (C). This is demonstrated in both tangential ( $a$ and $b$ ) and transverse ( $c$ and d) sections. The arrow in a denotes a perichromatin granule. The arrowheads in $\mathbf{b}$ indicate associations of polyribosomes with the outer annulus, whereas those in $\mathrm{c}$ and $\mathrm{d}$ point to the annular structures in nuclear envelope cross sections. Note that the fibrils of the "network of ribonucleoprotein elements" (sensu Busch and Smetana, 1970) appear to terminate at inner annuli (c) (a, $\times 80,000$, bar indicates $0.2 \mu \mathrm{m}$; $\mathrm{b}, \times 140,000$; $, \times 90,000$; $, \times 78,000$, bars in $\mathrm{b}$ and $\mathrm{c}$ indicate $0.1 \mu \mathrm{m}$ ). 


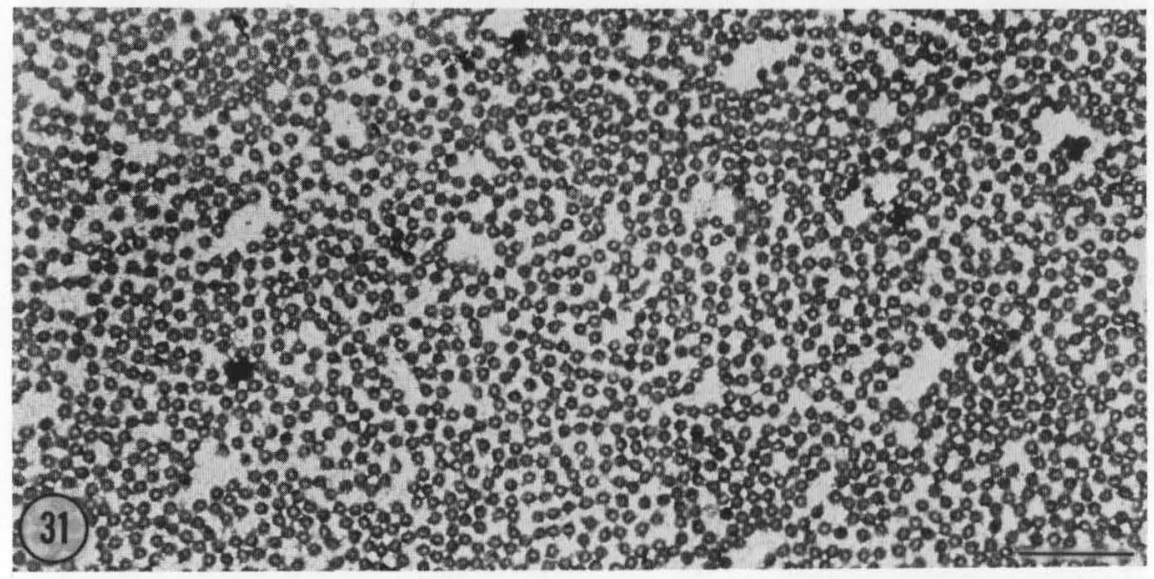

Fig. 31 Survey electron micrograph of a spread nuclear envelope isolated from a Xenopus laevis oocyte after treatment with pancreatic ribonuclease $(0.5 \mathrm{mg} / \mathrm{ml}$, SSC-buffer, $\mathrm{pH} 7.2,10 \mathrm{~min}$ at $25^{\circ} \mathrm{C}$, heated to $80^{\circ} \mathrm{C}$ at $\mathrm{pH} 5.0$ before use) and positive staining with acidic sodium phosphotungstate (compare Miller and Beatty, 1969). Pore complexes appear densely stained and substructures such as the central elements are still visualized ( $\times 12,000$, bar indicates $1 \mu \mathrm{m})$.

\section{Nuclear Pore Complex Patterns}

In most nuclei, pores seem at first glance to be distributed at random. However, when pore arrangement is studied in detail, significant deviations from randomness are noted; these are probably a consequence of the existence of a minimal pore distance (Maul et al., 1971). More conspicuous deviations from random arrangements have been observed as an irregular dense clustering or in the form of both hexagonal and square packaging (Drawert and Mix, 1961; Merriam, 1962; Wiener et al., 1965; Sichel, 1966; Wunderlich and Franke, 1968; Flickinger, 1970; Wecke and Giesbrecht, 1970; Folliot and Picheral, 1971; Kartenbeck et al., 1971; Thair and Wardrop, 1971; LaCour and Wells, 1972; Teigler and Baerwald, 1972; regular pore arrays are also common in AL: Kessel, 1968a; Scheer and Franke, 1969). Decorative row formations of pore complexes have also been mentioned for various nuclei (Northcote and Lewis, 1968; Neushul and Walker, 1971; Roberts and Northcote, 1971; Lott et al., 1972; LaFountain and LaFountain, 1973). Another clear case of nonrandomness of nuclear pore distributions is the confinement of pore complexes to certain nuclear regions and their total absence in others (LaCour and Wells, 1972). In spermatocytes and spermatozoa 
of various animals, mammals included, pores are absent in the sperm head and are restricted to the posterior (basal) "skirt" part, the diverticulum, which is free from condensed chromatin ("redundant nuclear envelope;" e.g. Franklin, 1968; Stanley, 1971a; Rattner and Brinkley, 1971; Wooding and O'Donnell, 1971; see these sources for further references, also see Section VII, J). Confinement of pores to small distinct nuclear regions is also observed in various other invertebrate and vertebrate differentiating sperm cells (Stanley, 1969, 1971b). They are also generally excluded from nuclear envelope areas adjacent to the (forming) acrosomal vesicle and to the centriole bases (Figs. 35 and 37). Absence of nuclear pore complexes has also been noted in those regions of the meiotic prophase nucleus which are tightly associated with nucleolar masses and the synaptinemal complexes (e.g. Meyer, 1963; LaCour and Wells, 1972; Scheer and Franke, 1972; Moses, 1960a, 1968; see, however, the concept of Engelhardt and Pusa, 1972), as well as in the deep invaginations in microsporocyte nuclei in a gymnosperm (Aldrich and Vasil, 1970) and in the evaginations of the eggs of a fern, Pteridium aquilinum (Bell, 1972).

A unique pattern of nuclear envelope organizations is seen in the dinoflagellate genus Noctiluca, where pore complexes occur only in the invaginated parts, the ampullae (Afzelius, 1963; Soyer, 1969a, b).

It is not clear whether such local accumulations or eliminations of nuclear pores are the result of any corresponding local heterogeneity in nuclear functions or nucleocytoplasmic interchange.

\section{N. Number and Frequency of Nuclear Pore Complexes}

The frequency of pore complexes per nuclear surface unit has been determined in a variety of nuclei (Afzelius, 1955; Barnes and Davies, 1959; Schnepf, 1960; Merriam, 1962; Moor and Mühlethaler, 1963; Branton and Moore, 1964; Wiener et al., 1965; Franke, 1966b, 1967a, b; Yoo and Bayley, 1967; Franke and Kartenbeck, 1969; Scheer and Franke, 1969; Wunderlich, 1969a, b; Comes and Franke, 1970; Girbardt, 1970; Schjeide, 1970; Speth and Wunderlich, 1970; Kartenbeck et al., 1971; Maul et al., 1971, 1972; Roberts and Northcote, 1971; Sprey and Hasche, 1971; Thair and Wardrop, 1971; Lott et al., 1972; Wunderlich and Speth, 1972; Hanzely and Olah, 1973; LaFountain and LaFountain, 1973; Scheer, 1973). The pore counts per membrane area unit, however, can be seriously influenced by the specific preparation method (see above), probably a consequence of the differences in shrinkage, distortion, or relaxation which can take place in the course of the isolation or the fixation and dehydration steps (Franke, 1970a). Moreover, the extent 

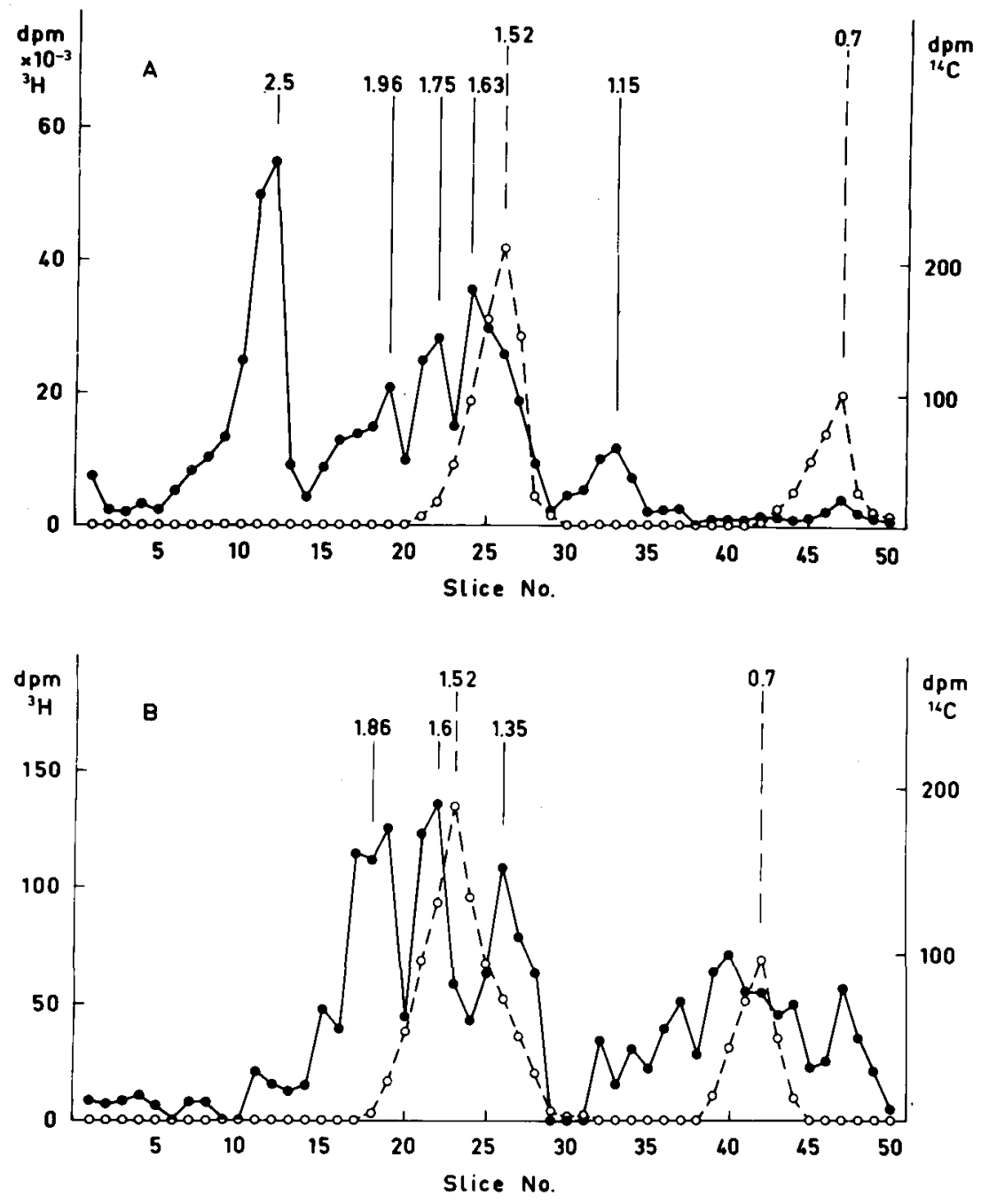

\section{2}

Fig. 32 Electrophoretic analysis of labeled RNA of two nuclear fractions, nucleoplasm (A) and nuclear envelopes (B). Selected lampbrush stage oocytes of Xenopus laevis were incubated in Eagle's medium ( $1: 1$ diluted with distilled water) containing $100 \mu \mathrm{Ci} / \mathrm{ml}$ each of tritiated uridine, cytidine, guanosine, and adenosine for 2 days at $25^{\circ} \mathrm{C}$; 50 nuclei were isolated and fractionated manually into the aggregated nuclear contents and nuclear envelopes (compare Fig. 3). The fractions were collected in ice-cold 70\% ethanol, pelleted, and kept cold. The RNA was extracted by incubating the pellets in $0.02 M$ Tris- $\mathrm{HCl}$ buffer ( $\mathrm{pH} 7.4$ ) containing $0.5 \%$ SDS and $1 \mathrm{mg} / \mathrm{ml}$ predigested pronase at $25^{\circ} \mathrm{C}$ for $10 \mathrm{~min}$. $\left[{ }^{14} \mathrm{C}\right] \mathrm{rRNA}$ was extracted from iso- 
to which this occurs is also variable from one nuclear type to another: amphibian oocytes, for instance, show no significant differences in pore frequency whether they are isolated and negatively stained, chemically fixed in situ, dehydrated and sectioned, or frozen and freeze-cleaved (Scheer, 1970a; Kartenbeck et al., 1971). In other nuclei such differences are dramatic, and it must be emphasized that comparisons between data obtained with different methods cannot be made. It is likely that the pore frequencies of nondehydrated nuclei fixed in situ are the closest to in vivo values. In any event, it is obvious that average pore frequencies differ greatly in different nuclei; with freeze-etch data, for instance, from ca. 1 pore $/ \mu \mathrm{m}^{2}$ up to ca. 60 .

In some cell differentiations which are characterized by progressive decrease in nuclear transcriptional activity pore frequency, total nuclear surface, and pore number decrease significantly (during spermiogenesis, during late amphibian and avian erythropoiesis, during "sporogenesis" in the dinoflagellate Noctiluca, in the nucleus of the generative cell of plant pollen tubes; see Werner, 1966; Soyer, 1969a; LaFountain and LaFountain, 1973). An increase in nuclear surface and also in pore frequency is correlated with the activation of nuclear transcription in lymphocytes (Tokuyasu et al., 1968; Maul et al., 1971). However, there are also examples of nuclear inactivation (i.e., decrease in nuclear RNA synthesis) which are not paralleled by a decrease in nuclear pore frequency, e.g., mature amphibian oocytes (Scheer, 1973). Pore frequency and pore number is likewise not considerably, if at all, diminished in the presence of antibiotic drugs inhibitory to RNA synthesis such as actinomycin D (Wunderlich, 1969b; Scheer, 1970b; Eckert et al., 1972), whereas De La Torre et al. (1973) report in onion root tip cells a decrease in pore frequency, concomitant with an enlargement of the perinuclear space, after $6 \mathrm{hr}$ immersion in the inhibitory drug, ethidium bromide. Consequently, one cannot generalize to regard nuclear pore frequency as an absolute indicator of the nuclear RNA synthetic activity. Periodic variations in nuclear pore frequency during the cell cycle

lated ribosomes from a $\left[{ }^{14} \mathrm{C}\right]$ uridine-labeled Xenopus laevis ovary, $20 \mu \mathrm{g}$ was added as a marker, and the RNA was precipitated by adding $\mathrm{NaCl}$ (to a final concentration of $0.1 \mathrm{M}$ ) and $2 \mathrm{vol}$ ethanol. The RNA pellet was suspended in $20 \mu \mathrm{l}$ of electrophoresis buffer containing $0.2 \%$ SDS and analyzed on slabs of $0.5 \%$ agarose-2.25\% acrylamide composite gels at $10 \mathrm{~V} / \mathrm{cm}$ (for further details see Ringborg et al., 1970). The RNA distribution of the nuclear envelopes (B) was corrected for a slight nucleoplasmic contamination as monitored by the presence of some pre-rRNA with a molecular weight of $2.5 \times 10^{1}$ daltons (less than $1 \%$ of the pre-rRNA in the nucleoplasmic fraction). The numbers above the peaks give the molecular weight in million daltons. Open circles indicate the ${ }^{14} \mathrm{C}$ radioactivity of the rRNA marker. 

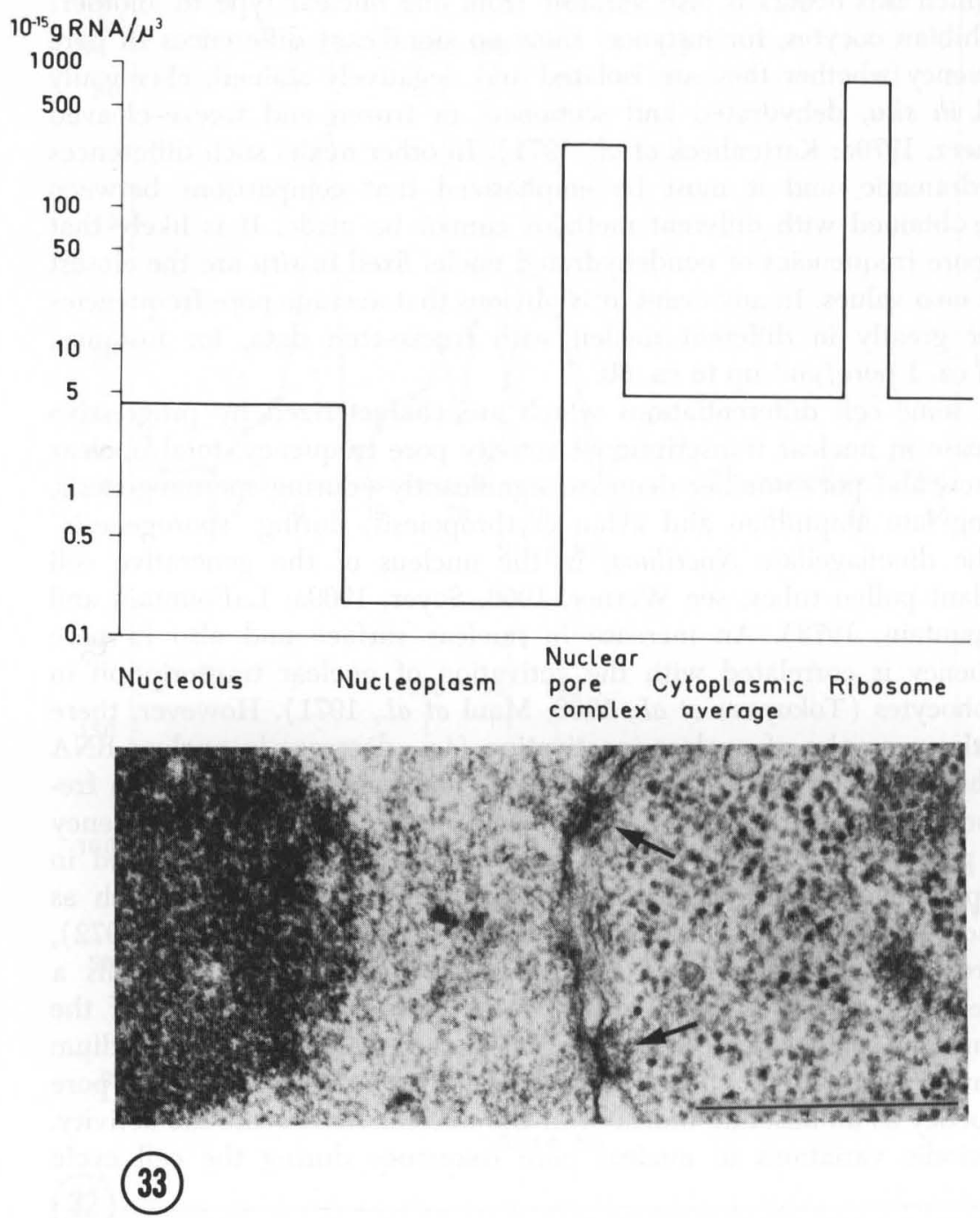

Fig. 33 RNA concentration $(\mathrm{w} / \mathrm{v})$ in the nuclear pore complexes, as compared to RNA concentrations of other intranuclear and extranuclear structures in a maturing Xenopus laevis oocyte. The RNA package in the nuclear pore complexes is lower (by ca. 33\%) than that of a ribosome but much higher than in other cellular RNP structures. The calculations were based on RNA determinations and volume estimations of the respective structures (for further details see Scheer, 1972). The electron micrograph illustrates the corresponding cytological situation in an ultrathin section through a lampbrush stage Xenopus laevis oocyte (arrows indicate pore complexes $)(\times 60,000$, bar indicates $0.5 \mu \mathrm{m})$. 
have been described (Wunderlich, 1969a; Scott et al., 1971; Maul et $a l ., 1972$ ). As a consequence of the relative constancy of the inner pore diameter, the percentage of nuclear pore area per nuclear surface area is also a relatively constant value in a given nucleus, provided that areas large enough to compensate for local patch heterogeneities are measured. This ratio, however, varies strongly among different cell types since it increases primarily with the pore frequency. A remarkable constancy of this percent pore area ratio has been noted in some cellular differentiation processes (oogenesis: Franke and Scheer, 1970b), as well as throughout the cell cycle (in synchronized Tetrahymena pyriformis GL: Wunderlich, 1969a; see, however, the data of Scott et al., 1971, and Maul et al., 1972), and for polyploidization (Sprey and Hasche, 1971).

\section{O. Nuclear Pore Complexes and Nucleocytoplasmic Translocation Processes}

Since their first description, nuclear pores have been discussed as important gateways controlling nucleocytoplasmic exchange of molecules and particles in either direction (see the reviews of Feldherr and Harding, 1964; Baud, 1965; Stevens and André, 1969; Feldherr, 1972; Franke, 1974). However, transport through the pores is not the only route via which nuclear material can enter the cytoplasm and vice versa. Several other pathways of nucleocytoplasmic transport of substances can be envisaged, and at least some of these alternatives have been demonstrated, in special cytological cases, in the electron microscope (Fig. 34). Another alternative, principally divergent from all these, would be that transient breakdowns of the nuclear envelope occur so that nuclear material can massively "leak out" into the cytoplasm (Schleusenmechanismus, Berg, 1932; for reviews see Clark,1960; Izquierdo and Vial, 1962; Schjeide, 1970; Blackburn, 1971; see also Tashiro et al., 1968), a situation corresponding to what happens in the "open" mitoses and meioses (see Section XII).

Various workers have approached the problem of nucleocytoplasmic exchange and nuclear envelope permeability by studying the uptake or release of substances in vitro with isolated nuclei (reviews: Feldherr and Harding, 1964; Stevens and André, 1969; Feldherr, 1972; Siebert, 1972; Franke, 1974). Such studies, however, cannot be regarded as relevant to the intact system since in isolated nuclei, except for giant nuclei prepared by hand with great care, the nuclear envelope is more or less punctured or disintegrated and the pore complex structures are altered so that one cannot assume a priori that one still deals with 


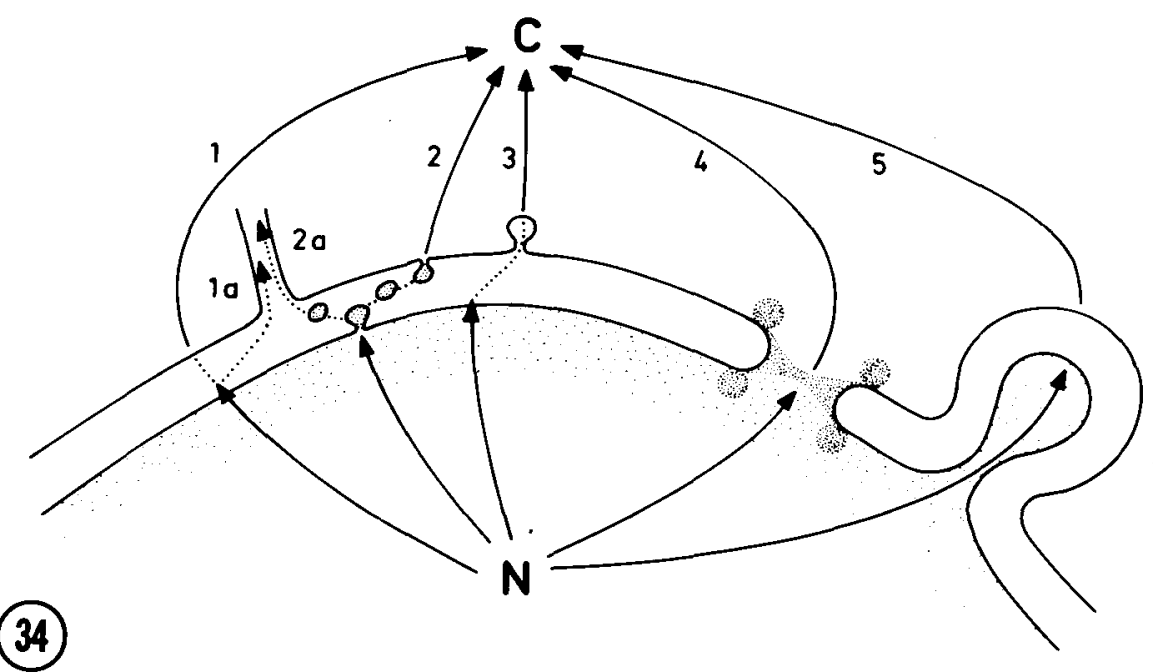

Fig. 34 Scheme of the possible pathways in which substances can be translocated from nucleoplasm (N) to the cytoplasm (C): (1) Transport through both nuclear membranes; ( Ia) transport through the inner nuclear membrane followed by intracisternal flow; (2a) invagination at the inner nuclear membrane, followed by vesicle formation and intracisternal translocation of the vesicle into the ER; (2) similar to 2a; however, with fusion of the intracisternal vesicle with the outer nuclear membrane and release of the vesicle content into the cytoplasmic ground substance; (3) transport through inner nuclear membrane followed by vesicle pinching-off from the outer nuclear membrane and vesicle flow into the cytoplasm; (4) migration through pore complexes; (5) formation of nuclear evaginations followed by detachment of the nuclear envelope surrounded buds.

a continuous nucleocytoplasmic barrier. Results of such experiments should be interpreted in terms of binding to nuclear components rather than in terms of transport across the envelope.

Experimental data relevant to the question of such exchange processes and the morphological routes involved are still very limited.

\section{IONS AND SMALL MOLECULES}

Ions and small molecules (below ca. 1000 daltons) can readily and rapidly exchange between nucleoplasm and cytoplasm. This has been known from the early studies of Abelson and Duryee (1949), Callan (1952) and Naora et al. (1962) and is particularly evident from the studies of Riemann et al. (1969) and of Horowitz's group (Horowitz and Fenichel, 1968, 1970; Century et al., 1970; Horowitz, 1972) as well as from the determinations by Siebert and his associates (reviewed in: Siebert and Langendorf, 1970; Siebert, 1972; see also Kohen et al., 1971). 
This seems to be in contrast, however, to the electrophysiological data of Loewenstein's group demonstrating a significant potential difference across the nuclear envelope in insect salivary glands (in the same order of magnitude as that across the cell membrane), but not in other cell types, as well as a correlation of changes of this nuclear envelope resistance to free ion permeability with certain stages in larval development (Kanno and Loewenstein, 1963; Loewenstein and Kanno, 1963a; b; Loewenstein, 1964; Ito and Loewenstein, 1965; Kanno et al., 1965; Wiener et al., 1965).

Intranuclear accumulation of ions and small solutes is frequently observed, but should be interpreted as an indication of binding to intranuclear structures or of a large free solvent space, as the latter is the case in large oocyte nuclei, instead of an indication of active transport across the nuclear envelope (for references see Century et al., 1970; Horowitz and Fenchel, 1970; Horowitz, 1972; Siebert, 1972).

Nothing can be said at the moment as to whether the movement of such small molecules and ions is primarily or exclusively via the pore complexes or whether they pass the perinuclear cisterna membranes at a comparable rate as well. There is also no evidence to exclude the possibility that molecular translocation occurs via vesicle blebs formed at the nuclear envelope which, after formation, could fuse with other vesicular or cisternal membranes or release their contents through membranolysis.

\section{LARGE MOLECULES AND PARTICLES}

A variety of large molecules and particles including proteins and ribonucleoproteins can enter or leave the nucleus. The transport is, however, selective and at different rates and with a size limitation. The time span after which labeled or metal atom-containing proteins appear in the nucleoplasm after injection into the cytoplasm is inversely related to the molecular weight (Gurdon, 1969, 1970; Paine and Feldherr, 1972). Charge differences might also be important since negatively charged particles seem to be preferentially bound by the pore complex material (Feldherr, 1964). Feldherr (1964, 1965, 1966) studied the distribution of colloidal gold particles, which in some experiments had been coated with an inert polymer, in amoeba nuclei after microinjection into the cytoplasm, and clearly demonstrated that an upper size limit for passage through the nuclear envelope exists (125-140 $\AA$ for the gold particles and even less, ca. $95 \AA$, for ferritin). These experiments further showed that (a) such large molecules and particles migrate only through the pores (De Robertis, 1954), and (b) that not the entire pore lumen 
(600-800 $\AA$ in diameter) is accessible for this passage but only a narrow central "channel" with a diameter of about 100 to $150 \AA$. Tangential sections of the nuclear envelope of such amoebae after injection showed the electron dense particles exclusively located in the very center of the pore (review: Feldherr, 1972).

Feldherr's results correspond to the report of Summers $(1969,1971)$, who showed that the nucleocapsids of a granulosis virus (diameter ca. $330 \AA$ ), after infection of midgut cells of the cabbage looper, Trichoplysia ni, do not enter the nucleus as a whole but attach end-on to the pore complex center and release their nucleic acid contents in a "phagelike" way through the pore into the nucleus, where replication takes place. The emptied virion capsids apparently remain associated with the cytoplasmic side of the pore complex. A similar interaction with the nuclear pore complexes has been shown for the infection of HeLa cells with some types of adenovirus (Morgan et al., 1969; Chardonnet and Dales, 1970). Transpore passage of viral particles in the opposite (nucleocytoplasmic) direction has been suggested in an article by DeZoeten and Gaard (1969) for the southern bean mosaic virus.

Feldherr's concept of a confinement of particle transpore passage to a narrow channel within the pore also finds support in the many descriptions of distinct large electron-dense aggregates which appear first in the nucleus, become then attached to the pore complexes and finally migrate into the cytoplasm. This has been shown for Balbiani ring-derived granules (diameter $300-500 \AA$ ) in the salivary glands of chironomids (Beermann, 1964; Stevens and Swift, 1966) as well as for the 200 to $700 \AA$ large aggregates in amphibian oocytes which have been suggested to have detached from the lampbrush chromosomes (Takamoto, 1966) or the nucleoli (Lane, 1967; Scheer and Franke, 1970b). Such large particles approach and bind to the nucleoplasmic side of the nuclear pore complexes, then elongate into rodlike structures with a waist diameter of ca. $150 \AA$ and appear to penetrate the pore, there by assuming an intermediate characteristic dumbbell-shape (Figs. 25 and 26; Beermann, 1964; Stevens and Swift, 1966; Scharrer and Wurzelmann, 1969a; Franke and Scheer, 1970b). Finally, this material rounds off again into a spheroid body and, at least in the oocytes, accumulates in the juxtanuclear zone where it is recognized as large, fused aggregates frequently closely associated with mitochondria (Figs. 25, 26). These may be RNP particles which are translocated into the cytoplasm (e.g. Beermann, 1964; Stevens and Swift, 1966; Scharrer and Wurzelmann, 1969a; Franke and Scheer, 1970b; Dhainaut, 1970a; see also Allen and Cave, 1968 and Cave and Allen, 1971; Vazquez-Nin and Bernhard, 1971) and tend to accumulate in the various forms of "heavy body-like" aggre- 
gates in the vicinity of the nuclear envelope (Pollister et al., 1954; Anderson and Beams, 1956; Ornstein, 1956; for the diverse synonyms see: Harris, 1967, 1969; Ulrich, 1969; Dhainaut, 1970a; Al-Mukhtar and Webb, 1971; Eddy and Ito, 1971; Franke and Scheer, 1971; Reverberi, 1972; Reverberi and DeLeo, 1972). Some recent cytochemical studies, however, rather suggest that they contain only little, if any, RNA (Clerot, 1968; Eddy and Ito, 1971; Gerin, 1971). In any event, it is a clear demonstration of a massive particle transport through pore centers. Somewhat similarly, transpore emission of the material contained in dense nuclear particles, apparently concomitant with an accumulation of densely staining aggregates in the juxtanuclear cytoplasm, seems to occur at the primary nucleus of Acetabularia (Figs. 2 and 24a). A similar transport through nuclear pores into the cytoplasm has also been sugested for two other nuclear structures which are likely to consist of RNP, namely the granulofibrillar "nuclear bodies" ("sphaeridia," "karyosomes"; see, e.g., Bouteille et al., 1967; Büttner and Horstmann, 1967; Rupec, 1969; Norberg, 1970), and the helices characteristic of some amoeba species (Stevens, 1967; Wise et al., 1972).

From this mode of nucleocytoplasmic migration of particulate material through a ca. $150 \AA$ broad central pore complex channel it has been suggested that the central elements of the nuclear pore complexes as seen in tangential sections and fractures or in isolated nuclear envelope fragments might generally represent a snap-shot of such material in statu transeundi (Stevens and Swift, 1966). This view of the central elements as a motile structure passing the pore has received further support from reports on quantitative differences in central granule frequencies between nuclei differing in RNA synthesis activity (Merriam, 1962; Wunderlich, 1969b, 1972; Franke and Scheer, 1970b; see, however, Eckert et al., 1972, and LaCour and Wells, 1972). Recently, however, it has become clear that granules cannot be regarded per se, at least not exclusively, as particles in nucleocytoplasmic migration: $(a)$ they occur in both intranuclear and cytoplasmic AL as well; $(b)$ their number is not drastically reduced in some nuclei very inactive in transcription such as maturing spermatocytes (Figs. 18d and 28) and oocytes (Scheer, 1972) and after drug inhibition of transcription (Eckert et al., 1972; in a correction of earlier reports based only on negatively stained preparations: Wunderlich, 1969b; Scheer, 1970b). Likewise, they are identified during intranuclear mitoses and meioses, including the macro- and micronuclear divisions of some ciliates (e.g., in Paramecium: Stevenson and Lloyd, 1971; Stevenson, 1972); (c) they are present in the foldedback nuclear envelopes of amphibian sperm cells (Fig. 28; see also Picheral, 1970), in the caudad nuclear envelope delaminations in sperma- 
tids (Rattner and Brinkley, 1971), and in the envelope fragments observed during various mitoses (see Section XII). Thus, the safest conclusion at the moment is that particles en route to the cytoplasm can contribute to the appearance of a central granule, but that a central granule can also represent an independent, static component of the pore complex which either might by RNP remaining attached to the pore complex after cessation of nucleocytoplasmic transport or might be a structure which has nothing to do with transpore migration (Franke and Scheer, 1971; Eckert et al., 1972; Franke, 1974).

There are also morphological indications that migration through the very pore center is perhaps not the only mode of migration through a pore complex. This is suggested from the described fibrilllar continuities of nucleolar and chromosomal structures, and aggregates derived therefrom, with the granules of the inner annulus (Fig. 27). Therefore, it might be that nuclear material, including RNP, is "spun out" from the nucleoli and/or the chromosomes into the cytoplasm in a fibrillar form (Kessel, 1968c; Franke and Scheer, 1970b) through both the pore periphery and the pore center (see Fig. 23b-f). That granules containing RNP do not generally pass the pore complex in a compact form is also demonstrated by the "perichromatin granules" (Watson, 1962) which approach the nuclear pores but then show only finely filamentous connections with the pore complex structures, so as to suggest that the material is unraveled before being transferred to the other side of the pore complex (Monneron and Bernhard, 1969; Vazquez-Nin and Bernhard, 1971).

In view of the above experimental evidence that the pore complex structures contain RNP, and the various indications that nucleocytoplasmic translocation of RNP containing particles and fibrils goes through pore complexes, it seems a reasonable hypothesis that the nonmembrane constituents of a pore complex might in general contain transitory RNP fixed in a membrane-bound state. This could hold for all the various situations discussed above if one includes the idea that the dissociation rate of such material from the membrane pore can be very variable in different cellular situations. In an actively synthesizing nucleus with a corresponding output of RNP into the cytoplasm, pore complex structures might in the first instance represent a steady state equilibrium structure, whereas after cessation of RNP transport pore complex structures are not released into the cytoplasm but remain stably associated with the membrane (Franke and Scheer, 1970b; Scheer, 1972, 1973).

There are no data at the moment as to which RNP species is associated with the pore complex. It is at least suggested from the dramatic increase in nuclear pore complexes during the lampbrush stage of am- 
phibian oogenesis, for instance (a stage primarily characterized by formation of ribosomal RNP; Davidson et al., 1964), that a good deal of the pore complex material may be ribosomal RNP ( $r R N P$; Verhey and Moyer, 1967; Franke and Scheer, 1970b; Scheer, 1972, 1973). This is suggested by gel electrophoretic separations of envelope-attached RNA* from such cells (Fig. 32), which show an accumulation of later stages in rRNA processing at the pore complexes, in agreement with the conclusions of various authors from the Chironomid salivary gland system (Ringborg and Rydlander, 1971), in nuclei from yeast (Sillevis Smitt et al., 1972) and amphibian oocytes (Scheer, 1973; Scheer et al., 1973), and in macronuclei of Tetrahymena pyriformis (Eckert et al., 1974), that final processing of rRNA takes place during or immediately after entrance into the cytoplasm. Thus, whether the pore complexes are not simply gateways for the entry of RNP into the cytoplasm, but also sites of final processing and assembly in ribosome formation (Franke and Scheer, 1970b), must be examined.

As for the other side of the pore complex it has been discussed that polyribosome formation begins at the outer annulus of the pore complex (Mepham and Lane, 1969; Franke and Scheer, 1970b; Jacob and Danieli, 1972 ), as suggested by the various associations of polyribosome chains with this annulus (see Figs. 24d and e, 27, 30). That the ribosomes of the nuclear envelope in toto are either transitory stages in the movements of cytoplasmic ribosomes, or have a higher turnover rate than cytoplasmic average ribosome, has been suggested from their kinetically intermediate character reported by some authors (Bach and Johnson, 1966; Smith et al., 1969; on the contrary, see Whittle et al., 1968). Furthermore, the time sequence of synthesis of a new polypetide observed after induction of antibody formation in plasma cells (Avrameas and Bouteille, 1968; Leduc et al., 1968; Avrameas, 1970) indicates that a newly produced messenger RNA is translated on nuclear envelope polyribosomes before it appears further out in the ER polyribosomes.

Nothing can be said at the moment as to whether messenger RNA, after addition of the poly (A) segment (for references see Adesnik et al., 1972) and assembly into "informosomes" for transport (Samarina et al., 1968; Spirin, 1969; Lukanidin et al., 1972), and the various small RNA species (5 S RNA, tRNA) are included in the pore complex material as well.

If one assumes that nucleocytoplasmic transport of RNA goes through

\footnotetext{
* It is not known whether, and in what amounts, nuclear envelopes contain the endogeneous membrane-bound (nonribosomal)RNA described for ER membranes (for reviews see: Pitot et al., 1969; Shapot and Davidova, 1971).
} 
the nuclear pore complexes, one can ask what is the flow rate of RNA through the average nuclear pore in a given cell (Franke, 1970b). Table I summarizes calculations of nuclear pore flow rates of RNA per average pore complex in different cell systems. The data illustrate that marked differences of RNA flow per pore can be found in different cell types and differentiation stages, and indicate that it is not only the number of nuclear pore complexes or their frequency per surface unit which is variable but also the flow rate of a specific substance through the pore complex (Franke, 1970b; Franke et al., 1971d; Wunderlich, 1972; Scheer, 1973).

In discussing the role of the nuclear pore complexes in nucleocytoplasmic transport processes it is important to direct attention to the cytochemical demonstrations of the presence of ATP-hydrolyzing activities (ATPase) not only in nuclear membranes in general but, markedly enhanced, in the nuclear pore complexes (Klein and Afzelius, 1966; Yasuzumi and Tsubo, 1966; Yasuzumi et al., 1967, 1968, 1969; Franke, 1973; for AL see also Scheer and Franke, 1969).

Mechanisms of nucleocytoplasmic translocations of large nucleoprotein particles not using the pore complex route have been clearly demonstrated in the course of the production of both DNA- and RNA-containing viruses such as herpesvirus (Darlington and Moss, 1968, 1969; Nii et al., 1968), a specific sweet-clover virus (Kitajima et al., 1969), and in the cells of an aphid insect after infection with sowthistle yellow vein virus (SYVV; Sylvester and Richardson, 1970). In this process, which is essentially similar to the pathway denoted by 2 and $2 a$ in Fig. 34, the nucleocapsid material is enveloped by inner nuclear membrane and then detaches into the perinuclear cisterna, thus having received a secondary coat derived from, though not identical with the inner nuclear membrane (for references see Ben-Porat and Kaplan, 1971). A somewhat similar blebbing mechanism has been proposed by Hinsch (1970) for the nucleocytoplasmic export of RNP-containing vesicles in the oocytes of a spider crab (see also Baud, 1965).

Another mechanism, which has been shown with particular clarity in oocytes and eggs, is that sketched under 5 in Fig. 34. This describes the formation of nuclear outpocketings, followed by membrane breakage and fusion in the neck region of this nuclear bleb, and detachment of a nuclear envelope-surrounded bit of nuclear contents. This seems to be the chief mechanism by which nucleolar material and nucleolar remnants are extruded from the nuclei of various gland cells and oocytes, and from pronuclei (Baud, 1953; Gay, 1955, 1956; Clark, 1960; Berendes and DeBruyn, 1963; Kessel and Beams, 1963; Bell and Mühlethaler, 1964; Szollosi, 1965; Baker and Franchi, 1969; Lima-de-Faria, 1971). After 
detachment of this nuclear envelope pocket, its membranes seem, at least in the oocytes of the house cricket, Acheta domesticus, to disintegrate and to release their contents into the cytoplasmic ground substance (Lima-de-Faria, 1971). A similar delamination of nuclear envelope evaginations has been described for the redundant nuclear envelope sections in various spermiogeneses (Stanley, 1971a; Rattner and Brinkley, 1971). It has also been hypothesized for the egg cells of the fern, Pteridium aquilinum (Bell and Mühlethaler, 1964; Bell, 1972), but it has been thought by these authors that the detached nuclear envelope sacs then differentiate into mitochondria (for comments see Section XI). Related mechanisms of nuclear outpocketing and bleb detachment, resulting in formation of a nuclear pore, have been proposed in studies by Hadek and Swift (1962) and Gulyas (1971; see this paper for further references). Kilarski and Jasinski (1970) derived from their electron micrographs of the fish gas gland cell a scheme in which a special form of nuclear bleb (with tubular infoldings from the inner nuclear membrane) becomes detached from the nucleus. A special and unique mode of extrusion of nuclear material has been discussed by Paweletz and Granzow (1972) for intranuclear glycogen which is found in various tumor cells (see Section II).

Except for the invasion of virus material, pathways of transport of cytoplasmic nuclei acids into the nucleus have hitherto not been demonstrated. Indications of the existence of a cytonucleoplasmic flow of specific RNA's, however, have recently been reported (Goldstein et al., 1973; Wise and Goldstein, 1973).

Comings and Okada (1972b) have recently revived the idea that the so-called chromatoid body, a cytoplasmic juxtanuclear clump, which is characteristic for spermiogenetic cells in a wide range of organisms, is also formed by an extrusion of nucleolar material, although their micrographs show neither direct transporous continuity of the two structures nor any indication of a corresponding nuclear bleb formation. This view strongly contrasts to that of Fawcett et al. (1970), who think that this body is an aggregate of cytoplasmic material (see also Fawcett, 1972; Schjeide et al., 1972). A related structure appears to be the "polar granule" which has been described in oocytes and eggs of some insects, particularly clearly in Drosophila (Mahowald, 1971a, b). These dense aggregates, possibly containing RNP, also accumulate during certain stages of egg development at the nuclear surface.

There is no information as to the route which proteins take in their nucleocytoplasmic exchange, except for the experiments of Feldherr (references quoted above; review: Feldherr, 1972) using ferritin and gold particles which were coated with protein-mimicking polymers. The 


\begin{tabular}{|c|c|c|c|c|c|c|c|}
\hline Cell system & $\begin{array}{l}\text { Electron } \\
\text { microscopic } \\
\text { preparation }\end{array}$ & $\begin{array}{l}\text { Number of } \\
\text { pores } / \mu \mathrm{m}^{2}\end{array}$ & $\begin{array}{l}\text { Nuclear } \\
\text { diameter } \\
(\mu \mathrm{m})\end{array}$ & $\begin{array}{l}\text { Total number } \\
\text { of pore } \\
\text { complexes } \\
\text { per nucleus }\end{array}$ & $\begin{array}{l}\text { Calculated } \\
\text { production } \\
\text { rate of } \\
\text { cy toplasmic } \\
\text { rRNA } \\
\text { (pg/hr/cell) }\end{array}$ & $\begin{array}{l}\text { NPFR } \\
\mathrm{N} \rightarrow \mathrm{C} \\
\left(10^{-18} \mathrm{gm}\right. \\
\mathrm{rRNA} / \\
\text { pore } / \mathrm{min})\end{array}$ & $\begin{array}{c}\text { NPFR } \\
\mathrm{N} \rightarrow \mathrm{C} \\
\text { (mole- } \\
\text { cules } \\
\text { rRNA/ } \\
\text { pore/ } \\
\text { min) }\end{array}$ \\
\hline \multirow[t]{5}{*}{$\begin{array}{l}\text { Mature rat } \\
\text { hepatocytes }\end{array}$} & $\begin{array}{l}\text { Nuclei fixed in situ, } \\
\text { freeze-etched }\end{array}$ & $\begin{array}{l}14.1 \pm 2.3 \\
\text { (a) }\end{array}$ & $\begin{array}{l}8.10 \\
\text { (b) }\end{array}$ & $2.91 \times 10^{3}$ & $0.20(\mathrm{c})$ & 1.14 & 0.56 \\
\hline & $\begin{array}{l}\text { Nuclei fixed in situ, } \\
\text { ultrathin sectioned }\end{array}$ & $\begin{array}{l}16.3 \pm 1.5 \\
\text { (a) }\end{array}$ & $\begin{array}{l}8.04 \\
\text { (b) }\end{array}$ & $3.31 \times 10^{3}$ & & 1.00 & 0.49 \\
\hline & $\begin{array}{l}\text { Isolated nuclei, } \\
\text { fixed, freeze-etched }\end{array}$ & $\begin{array}{l}24.9 \pm 3.0 \\
\text { (a) }\end{array}$ & $\begin{array}{l}8.02 \\
\text { (b) }\end{array}$ & $5.03 \times 10^{3}$ & & 0.66 & 0.32 \\
\hline & $\begin{array}{l}\text { Isolated nuclear } \\
\text { membranes, fixed, } \\
\text { freeze-etched }\end{array}$ & $\begin{array}{l}24.3 \pm 7.5 \\
\quad \text { (a) }\end{array}$ & 8.02 & $4.91 \times 10^{3}$ & & 0.68 & 0.33 \\
\hline & $\begin{array}{l}\text { Isolated nuclear } \\
\text { membranes, fixed, } \\
\text { neg. stained }\end{array}$ & $\begin{array}{l}35.8 \pm 4.3 \\
\text { (a) }\end{array}$ & 8.02 & $7.23 \times 10^{3}$ & & 0.46 & 0.23 \\
\hline \multirow[t]{2}{*}{$\begin{array}{l}\text { HeLa cells, expo- } \\
\text { nentially growing }\end{array}$} & $\begin{array}{l}\text { Isolated nuclear } \\
\text { membranes, fixed, } \\
\text { neg. stained }\end{array}$ & $46 \pm 7(d)$ & $\begin{array}{l}9.1 \\
\text { (d) }\end{array}$ & $11.97 \times 10^{3}$ & $1.4(\mathrm{e})$ & 1.95 & 0.96 \\
\hline & $\begin{array}{l}\text { Nuclei fixed in situ, } \\
\text { ultrathin sectioned }\end{array}$ & 25 & 9.1 & $6.5 \times 10^{3}$ & & 3.58 & 1.76 \\
\hline $\begin{array}{l}\text { Macronuclei of } \\
\text { Tetrahymena }\end{array}$ & $\begin{array}{l}\text { Nuclei fixed in situ, } \\
\text { freeze-etched }\end{array}$ & $38 \pm 9(f)$ & 12.0 & $17.19 \times 10^{3}$ & $131.2^{c}(\mathrm{~g})$ & 127.20 & 75.85 \\
\hline $\begin{array}{l}\text { pyriformis GL, } \\
\text { exponentially }\end{array}$ & $\begin{array}{l}\text { Nuclei fixed in situ, } \\
\text { ultrathin sectioned }\end{array}$ & $69 \pm 10(\mathrm{f})$ & 12.0 & $31.21 \times 10^{3}$ & & 70.06 & 41.78 \\
\hline growing & Isolated nuclear & $110 \pm 25(f)$ & 12.0 & $49.76 \times 10^{3}$ & & 43.94 & 26.20 \\
\hline
\end{tabular}




Xenopus laevis
oocytes, growth
interval from 300
to $1100 \mu \mathrm{m}$ oocyte
diameter (lamp-
brush stage)
Xenopus laevis oocytes, mature
Isolated nuclear membranes, fixed, neg. stained

membranes, fixed, neg. stained

Nuclei fixed in situ, freeze-etched

Nuclei fixed in situ, ultrathin sectioned Isolated nuclei, fixed, ultrathin sectioned Isolated nuclear membranes, fixed, neg. stained

$\begin{array}{llllll}60 \pm 4(\mathrm{a}) & 360.0^{d} & 24.43 \times 10^{6} & 4170.0(\mathrm{~h}) & 2.85 & 1.56 \\ 60 \pm 8(\mathrm{a}) & 360.0^{d} & 24.43 \times 10^{6} & & 2.85 & 1.56 \\ 67 \pm 3(\mathrm{a}) & 360.0^{d} & 27.28 \times 10^{6} & & 2.55 & 1.40 \\ 61 \pm 8(\mathrm{a}) & 360.0^{d} & 24.84 \times 10^{6} & & 2.80 & 1.53 \\ & & & & & \\ \begin{array}{c}47 \pm 3.3 \\ \text { (h) }\end{array} & 510.0 & 38.41 \times 10^{6} & 0 & 0 & 0\end{array}$

a Key to references:

(a) Kartenbeck et al. (1971).

(b) Franke et al. (1971d).

(c) Franke et al. (1971d; compare also Quincey and Wilson, 1969).

(d) Comes and Franke (1970).

(e) Compare the data of Seed (1966).

(f) See Franke (1967b); Wunderlich (1969a); Speth and Wunderlich (1970).

(g) Eckert (1972); see also Scherbaum (1957).

(h) Scheer $(1970,1973)$.

${ }^{b}$ Assuming a mean molecular weight of $1.225 \times 10^{6}$ daltons for rat and HeLa rRNA, of $1.0 \times 10^{6}$ for Tetrahymena rRNA and $1.1 \times 10^{6}$ for Xenopus ovary rRNA (Loening, 1968).

c The value includes the rRNA degraded per hour.

${ }^{d}$ At the middle of the oogenesis interval. 
translocation of some proteins from the nucleus into the cytoplasm, and vice versa, seems to be controlled. However, there are also various proteins which seem to distribute in a rather unrestricted manner between both intracellular spaces. The translocation control is specific, and probably biologically meaningful, insofar as some proteins are taken up by the nucleus at a high rate, whereas others need much longer times to cross the nuclear envelope, and another group of proteins remains totally excluded from the nucleus (surveys in: Feldherr and Harding, 1964; Goldstein 1964, 1970b; Goldstein and Prescott, 1967a, b; Feldherr, 1972; Legname and Goldstein, 1972; Paine and Feldherr, 1972; Siebert, 1972). This control seems to be primarily by size (molecules with diameters less than $45 \AA$ are able to enter the nucleus, proteins larger than $95 \AA$ appear to be excluded) and charge (Paine and Feldherr, 1972). Nuclear concentration of a protein needs not to involve specific transport but might be explained simply by its binding to a special nuclear component (Gurdon, 1970; Paine and Feldherr, 1972; for the special and still unsolved question of the uptake of hemoglobin into the nucleus see Small and Davies, 1970; Zentgraf et al., 1972; Brachet et al., 1973). Some proteins seem to be capable of rapidly shuttling between nucleus and cytoplasm with a strongly preferential localization within the nucleus (see the above-quoted references of Goldstein's group). It has to be concluded from the fact that most, if not all, nuclear proteins are synthesized on cytoplasmic polyribosomes (for references see Robbins and Borun, 1967; Goldstein 1970b; Wu and Warner, 1971; Bouteille, 1972) that mechanisms of rapid and specific uptake into the nucleus, as well as exclusion mechanisms, exist also for the endogenous cellular proteins. Such mechanisms must also be required for the cytonucleoplasmic flow of newly synthesized viral proteins in an infected cell (Ben Porat et al., 1969). The rates of the nucleocytoplasmic translocation of a protein, however, appear to vary between different nuclear types (Feldherr, 1969) and during the cell cycle (Feldherr, 1966) as well as during processes of differentiation and dedifferentiation (Merriam, 1969; Carlsson et al., 1973).

Although still very scarce, the present experimental results suggest that the control over the nucleocytoplasmic exchange of large molecules and particles is, in addition to size limitations and charge effects, controlled by the properties of the nuclear envelope itself. The regulatory parameters, however, are certainly not the nuclear pore diameter and the pore frequency (and, of course, not the resulting pore area per nuclear surface area): Feldherr (1969) rather favored the hypothesis that the pore complex contents, especially "the annular material might 
regulate cellular activity by controlling the passage of macromolecules across the nuclear envelope."

\section{Relationship of the Nuclear Envelope to Annulate Lamellae (AL) and Intranuclear Cisternae}

Pore complexes are not structures which are confined to the nuclear envelope. Their occurrence in the AL makes clear that one cannot regard them as being structures functioning only in nucleocytoplasmic compartmentalization and transport. There appear to be no differences in the pore complex ultrastructure of $\mathrm{AL}$ and nuclear envelope. However, the pore density in the AL can be much higher than in the nuclear envelope of the same cell (Hertig and Adams, 1967; Scheer and Franke, 1969; Maul, 1970a; Scheer and Franke, 1972). Formation of AL in the cytoplasm (in some cases into enormous stacks) provides additional evidence that the capacity for pore complex formation is not confined to a specific nuclear structure (see below). On the other hand, however, the occurrence of intranuclear AL demonstrates that any proposed inducer of pore complex formation is also not an exclusively cytoplasmic component.

The literature on cytoplasmic and intranuclear $\mathrm{AL}$ in animal and plant cells has been extensively reviewed (Kessel, 1968a; Wischnitzer, 1970; Scheer and Franke, 1972; for plant cells see also Gianordoli, 1969). It has been found that cytoplasmic AL cisternae can be continuous with the outer nuclear membrane (Figs. $7 \mathrm{~d}$ and $8 \mathrm{c}$ ), sometimes giving the impression that perinuclear AL are simply local proliferations of the nuclear envelope (Hsu, 1963, 1967; Frasca et al., 1967; Hertig and Adams, 1967; Kessel, 1968a; Wischnitzer, 1970). Correspondingly, intranuclear AL have been shown to be continuous with the inner nuclear membrane (Hsu, 1967; Everingham, 1968a, b; Folliot, 1968; Kessel, 1968a; Ollerich and Carlson, 1970; Fiil and Moens, 1973).

It is not clear how AL arise. Some authors hold the view that the pore complexes are formed in (rough) ER cisternae (Merriam, 1959; Rebhun, 1961; Mancuso, 1964; Hoage and Kessel, 1968; Franke and Scheer, 1971; Scheer and Franke, 1972; compare also Orci et al., 1972). Others have developed the concept that AL are delaminations of the perinuclear cisterna (e.g. Harrison, 1966; Hsu, 1967; Bal et al., 1968; King and Fordy, 1970; Merkow et al., 1970; ApGwynn et al., 1971; Mancuso, 1972). Formation of cytoplasmic AL by coalescence of vesicles pinched off from the outer nuclear membrane has also been discussed 
for some time (Kessel, 1963), but the earlier suggestive pictures have recently been interpreted as having resulted from a fixation artifact (Kessel, 1969b). Intranuclear cisternae with or without pore complexes do not always appear, however, as infoldings of the inner nuclear membrane. Isolated cisternae or vesicles are found in the nuclei of various cell types and frequently are associated with blocks of condensed chromatin or with layers of electron-dense material, possibly containing RNP (Yasuzumi and Sugihara, 1965; Folliot, 1968; Calarco and Brown, 1969; Jollie, 1969; Maul, 1970b; Merkow et al., 1970; Ollerich and Carlson, 1970; Franke and Scheer, 1971, Gulyas, 1972a, b; Legrand and Hernandez-Verdun, 1971; Roberts and Northcote, 1971; Haynes and Davies, 1973). Associations of intranuclear membrane formations, including vesicular ones, with the nucleolus are also not uncommon (Terzakis, 1965; Miller, 1966; Kessel and Beams, 1968; Balbai et al., 1969; Longo and Anderson, 1969; Dhainaut, 1970b; Ollerich and Carlson, 1970; Kezer et al., 1971; Franke et al., 1972a; Zibrin, 1972). Sometimes intranuclear cisternae are close to and parallel with the nuclear envelope, having densely stained material sandwiched in between them (e.g., Munk and Waldeck, 1969; Maul, 1970b; Franke and Scheer, 1971; Rowley et al., 1971; for further references see the review of Blackburn, 1971). Accumulation of intranuclear membrane profiles is particularly frequent in cell pathological stages and after drug treatments (for references see David, 1964; Döbel, 1970; Blackburn, 1971; Romen and Bannasch, 1973).

The functions of such intranuclear membranes remain obscure. Hinsch (1970) has ascribed to special intranuclear vesicles a role in nucleocytoplasmic transfer processes. Paweletz and Granzow (1972) have described a membraneous envelope surrounding aggregates of intranuclear glycogen in an Ehrlich-ascites cell line and have discussed an involvement of such membranes in the extrusion of this glycogen into the cytoplasm; this interpretation is, however, somewhat at variance with that of Karasaki (1971). The modes of formation of such intranuclear vesicles or cisternae are also unclear and may even be different in different situations. Maul (1970b) has favored the notion that, in cultured melanoma cells, such intranuclear cisternae or AL represent remnants of the perinuclear cisterna which after mitotic breakdown of the nuclear envelope become entrapped in the daughter nucleus during the reconstitution of the envelope (see also Calarco and Brown, 1969, for mouse embryonic mitoses; Schwalm, 1969, for early cleavage stages of the migratory locust; Roberts and Northcote, 1971, for cultured plant cells; and Szollosi et al., 1972a, for human oogonia). That this is, however, not the only mechanism of formation of intranuclear cisternae is best demonstrated in various oogeneses, most clearly in tunicates and some 
insects (mosquitoes), where they appear without a preceding nuclear division (Hsu, 1963, 1967; Kessel, 1964; Mancuso, 1972; Fiil and Moens, 1973).

An interesting observation which might be relevant to the principal question of pore complex formation has recently been communicated by Fiil and Moens (1973) in a study of mosquito oogenesis. These authors observed in the periphery of the karyosphere (i.e., the aggregate of all chromosomes) dense annular structures, particularly in association with the lateral element equivalents in these multiple synaptinemal complexes (see also Engelhardt and Pusa, 1972). Such annular formations, some of them containing a central granule, strongly resemble nuclear pore complexes but are connected to each other only by filamentous material. Intranuclear $\mathrm{AL}$ are not observed in these nuclei until later stages of oocyte maturation, and the situation might indicate that the membrane material of the cisterna between the pore complexes becomes assembled at the annular structures. If this could be proved it would make a provocative alternative (Franke and Scheer, 1971) to the most commonly held view that pore complexes only form in preexisting cisternae (see Section VII).

\section{Structural Differentiations of the Nuclear Envelope}

Several situations are known in which the structure of the nuclear envelope is locally altered. At least some of these alterations appear to be functionally important. In many spermatids, the region of the nuclear envelope which is adjacent to the acrosomal vesicle field or to the acrosomal cap itself is conspicuously altered (Fig. 35): The perinuclear space is narrowed, sometimes down to approximately $70 \AA$, is less flexible in outline, and the surface of the outer membrane or of the both nuclear membranes is closely associated with a layer of coarse, highly stainable, sometimes granularly substructured material (Rebhun, 1957; Fawcett, 1958; Horstmann, 1961; Bröckelmann, 1963; Werner, 1966; Barker and Biesele, 1967; Horstmann and Breucker, 1969; deKretser, 1969; Langreth, 1969; Fawcett and Phillips, 1970; Longo and Anderson, 1970; Sandoz, 1970; Fawcett et al., 1971; Plöen, 1971; Stanley, 1971a; Picheral, 1972a; Rattner, 1972). The nature of this apposed material has neither been identified nor is the specific reason for this localized nuclear envelope differentiation known. A similar transition characterized by the apposition of dense material on either nuclear membrane has been noted in the posterior regions of spermatid nuclei which face the basis and the adjuncts of the proximal centriole (and the associated 


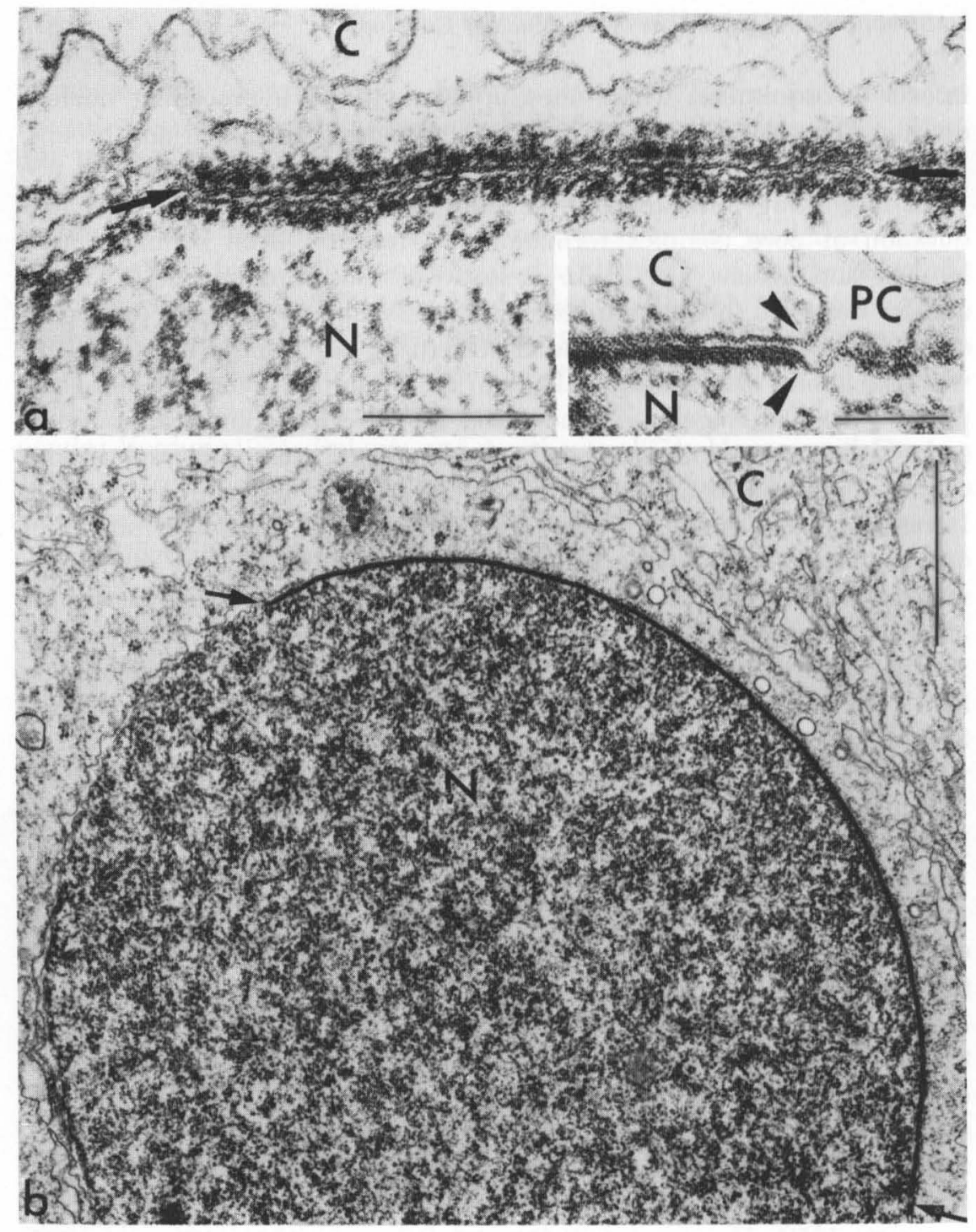

35

Fig. 35 Local differentiation of the nuclear envelope region which is adjacent to the vesicle field involved in formation of the acrosomal cap in spermatids of the snail, Helix pomatia. In this region (between the two arrows in b) the nuclear envelope is altered in that the perinuclear cisterna (indicated by the arrows in a) is less undulated, much narrower, devoid of pore complexes, and associated on either side with a layer of coarse, densely stained granules. The inset of a shows the very transition of "normal" perinuclear cisterna (PC) into this altered section (arrowheads). N, nucleus; C, cytoplasm ( $\mathrm{a}, \times 110,000$, bar indicates $0.2 \mu \mathrm{m}$; inset, $\times 136,000$, bar indicates $0.1 \mu \mathrm{m} ; \mathrm{b}, \times 22,000$, bar indicates $1 \mu \mathrm{m})$. 
connecting piece) or of the axial midpiece (Fig. 37; Werner, 1966; Reger, 1967, 1969; deKretser, 1969; Fawcett and Phillips, 1970; Phillips, 1970; Rattner and Brinkley, 1971; Stanley, 1971a; Starke, 1971; Fawcett, 1972; Picheral, 1972b; MacKinnon and Abraham, 1972; compare also the "nuclear plate" of Rattner, 1972). Similar local nuclear envelope differentiations have been noted in the regions which lie close to, and are associated with, the centriole-equivalent aggregate of an alga, the diatom Lithodesmium undulatum (Manton et al., 1969a and b), and in the "initial plaque" and the "growing papilla" cone described in coccid spermiogenesis by Moses and Wilson (1970). Close spacing of the perinuclear cisterna and increase in osmiophila is also characteristic for the attachment sites of the synaptinemal complexes (Moses, 1960a, 1968). Frequently a loss of definition in the membrane profile itself accompanies such alterations. This might reflect a local phase transition of the molecular architecture and/or a change in the membrane composition during limited cisternal collapse.

In the posterior regions of the mammalian sperm head it has been observed that, in the postnuclear cap, the narrowing of the perinuclear space can proceed to the extent that the two membranes come in contact or even fuse (Wooding and O'Donnell, 1971). Langreth (1969) in studies on the cancer crab spermiogenesis presented micrographs which show an apparently further step in nuclear envelope collapse: not only has the acrosome-adjacent envelope region become indistinct and very osmiophilic but one also sees true large gaps and vesicular fragmentation. Thus it might be generalized that these localized collapses of the perinuclear cisterna, concomitant with alterations of the internal membrane architecture, are intermediate stages toward the complete disintegration of the nuclear envelope as found in the sperm maturation of some coccids (Moses and Colemann, 1964; Robison, 1966; Moses and Wilson, 1970) and some other insects (see also Yasuzumi and Ishida, 1957, and Yasuzumi et al., 1971). Kessel (1966, 1970) has described in dragonfly spermatids a close association between microtubular bundles and regularly spaced longitudinal furrows in the nuclear envelope. In cross section it is these invaginated parts which show the collapsed and densified appearance of the perinuclear cisterna. The nuclear condensation then accompanies the retraction of the altered envelope regions, resulting in a flowerlike pattern of normal and collapsed intercepts in transverse sections.

A localized flattening of the perinuclear cisterna together with the formation of a fuzzy fibrillar coat and an increased stainability has been noted in the spermatids of the earthworm, Eisenia foetida (Stang-Voss, 1970; Stang-Voss and Staubesand, 1970). Such flattened envelope regions 
face a flattened cytoplasmic cisterna or a mitochondrion and the fibrillar coat seems to fill the interspace between the two adjacent membrane surfaces. In their interpretation these authors suggested that such lamellar arrangements might be involved in Golgi apparatus development and mitochondria formation from the nuclear envelope. Localized formations of nuclear membrane-associated lamellar stacks in ovarial cells of the bat, Tardaria brasiliensis cynocephala, (Ruby and Webster, 1972; see above) were also interpreted as giving rise to the formation of dictyosomes. Mycologists have also noted increased electron opacity, coincident with cisternal collapse, in the nuclear envelope-ER cisterna associations of the characteristic nuclear blebs that occur in late stages of ascospore formation. These blebs have been repeatedly implicated in the production of the ascospore-delimiting membranes (Carroll, 1967, 1969; Beckett and Crawford, 1970; Wells, 1972). Localized cisternal transitions of the type described are, however, in our opinion not limited to the nuclear envelope. They are related to the localized cisternal collapse phenomena in the ER such as the formation of the "lamellar bodies" in the rough ER of neuronal cells (Le Beux, 1972).

Nuclear envelope differentiations which are also characterized by reduced membrane distinctiveness, association with electron-dense material, and sometimes localized cisternal collapse, are found in a variety of organisms as pole-determining structures in intranuclear mitoses and meioses. In these examples the differentiated nuclear membrane regions act as polar "microtubule-organizing centers" (MTOC, Pickett-Heaps, 1969 ) for the spindle apparatus. The extent to which the nuclear envelope is structurally altered, however, is variable. For instance, in some lower fungi such as in Saprolegnia, Catenaria, and Blastocladiella it appears as a thickening of the inner nuclear membrane, usually in an indentation (pocket) of the envelope which corresponds to the position of the centrioles on the outer side (Heath and Greenwood, 1968, 1970; Ichida and Fuller, 1968; Lessie and Lovett, 1968; Howard and Moore, 1970 ). Similar polar changes in membrane appearance have recently been observed in the mitosis of the centriole-possessing xanthophycean alga, Vaucheria (Ott and Brown, 1973). In the acentriolar zygomycete, Phycomyces blakesleeanus, one finds apposed to the inner nuclear membrane a marked polar knob (diameter up to $0.1 \mu \mathrm{m}$, maximally $600 \AA$ thick) at which the spindle microtubules insert (Fig. 36a; Franke and Reau, 1973). Among the higher fungi, the Ascomycetes have characteristic "plaques," sometimes revealing a fibrillar texture and a complex subarchitecture consisting of two to three discs. Such polar plaques (some synonyms: centriolar plaque, centrosome, archantosome, centrosomal plaque) are either closely apposed to the outer nuclear membrane or to both (Wells, 1970; Beckett and Crawford, 1970; Zickler, 1970) or, 
as in the yeasts, are totally embedded into the envelope, thus resembling a porelike interruption filled with indistinct dense material (Moor, 1966, 1967; Robinow and Marak, 1966; McCully and Robinow, 1971; Moens and Rapport, 1971; Unger et al., 1971; for meiosis see Peterson et al., 1972). Such polar plaques serve not only as terminal foci of microtubular orientation for both the nucleoplasmic spindle apparatus and the cytoplasmic aster, but also, according to Zickler (1970), might be penetrated by such microtubules.

Plaquelike differentiations within the nuclear envelope resembling those of the Ascomycetes have been described in some protozoa such as the malarial parasites (Aikawa et al., 1967; Terzakis et al., 1967; Aikawa and Beaudoin, 1968; Scalzi and Bahr, 1968), hypermastigid flagellates (Hollande and Valentin, 1968), and radiolarians (Hollande et al., 1969 ). Some authors have suggested that such polar plaques might represent a specialized pore complex (Robinow and Marak, 1966; Scalzi and Bahr, 1968; Unger et al., 1971; Peterson et al., 1972. However, in spite of the fact that at first view they can resemble porous interruptions in the perinuclear cisterna, the micrographs published do not allow one to visualize them as pore complexes as defined in the previous Sections.

Recently, Zickler (1973) has presented cytochemical evidence for the presence of DNA in the plaque region, an observation that is hard to interpret at the moment and certainly will revive the perpetual debate concerning the association of small amounts of DNA with centrioles and centriole-equivalent structures (reviews: Fulton, 1971; Wolfe, 1972).

Cisternal proliferations from the outer nuclear membrane have already been introduced as continuities with ER and AL or as "short circuit" connections of the perinuclear cisternae (see Section IV), and the amplexus extensions have also been mentioned. A somewhat different specialization of an outer nuclear membrane proliferation is known in the "redundant nuclear envelope" of the posterior part of the mammalian spermatid nucleus (e.g. MacKinnon and Abraham, 1972). In this case the outer membrane-connected cisternae participate, together with microtubules, in the construction of the caudal tube (the "manchette").

A highly regular, intricate system of perinuclear cisternae has been described by Beams and Sekhon (1969) in the unicellular organism, Lophomonas blattarum. Here, sites of continuity of the nuclear envelope with rough ER cisternae are abundant and somewhat regularly spaced. Each of the rough ER elements radiates from the nucleus and, in the basal nuclear portion, ends in a smooth-surfaced ER ampulla so that the whole arrangement constitutes a perinuclear corolla of $(i)$ a proximal sheath of rough ER character and (ii) a more distal shell of exclusively smooth membrane sacs.

The dramatic reduction of nuclear volume and surface which takes place 

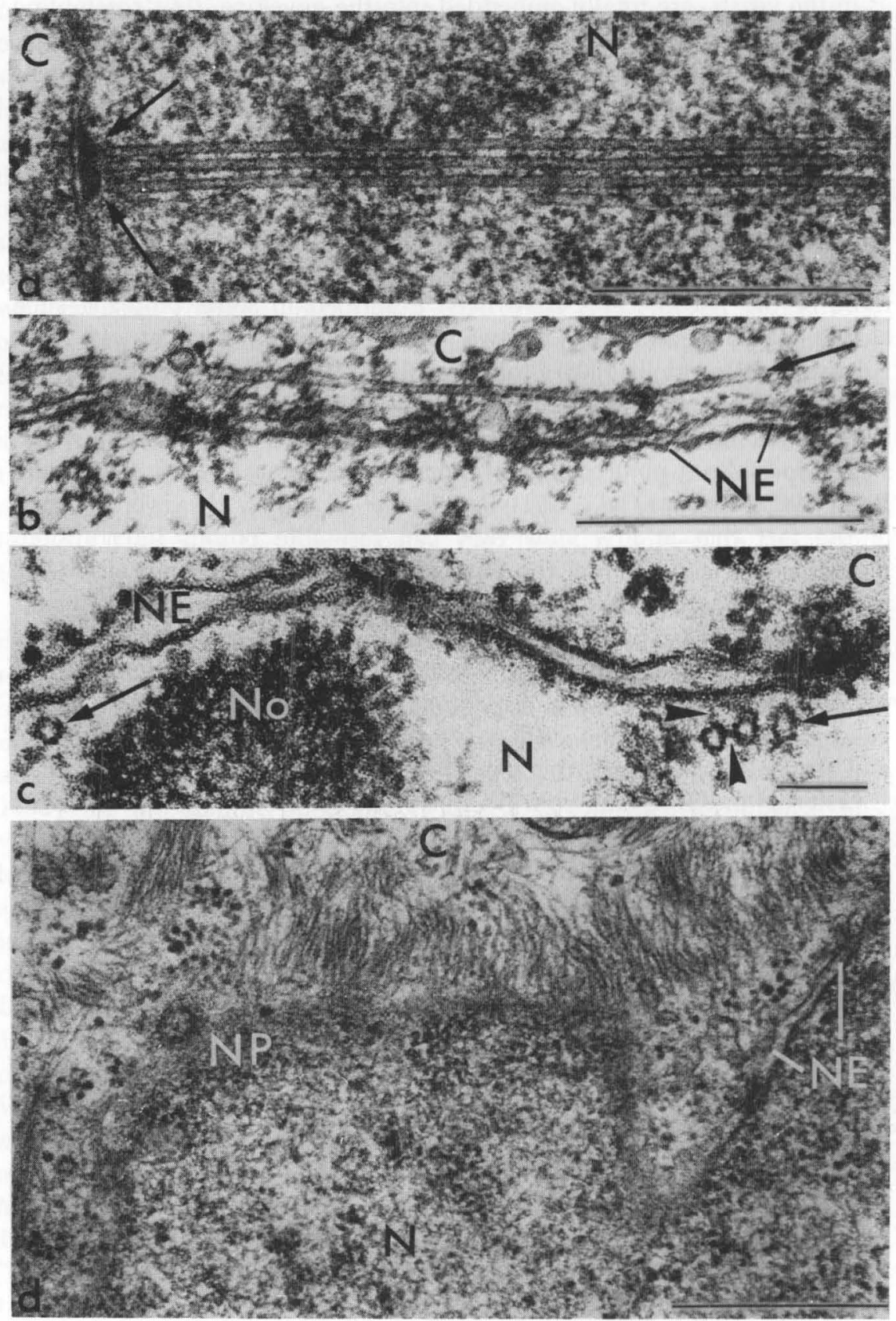
during sperm maturation leads to a corresponding reduction in nuclear envelope material. The part of the nuclear envelope which is not apposed to the condensing nuclear DNP and which, in contrast to the sperm head nuclear envelope, maintains the pore complexes and forms an undulating skirt surrounding the insertion groove of the centriole or the equivalent piece of "redundant nuclear envelope" (Yasuzumi, 1956; Horstmann, 1961; Bröckelmann, 1963; Werner, 1966; deKretser, 1969; Stanley, 1969, 1971a; Fawcett, 1970; Plattner, 1971; Rattner and Brinkley, 1971; Wooding and O'Donnell, 1971; see also MacKinnon and Abraham, 1972). Furthermore, the reduction of the spermatid nuclear envelope involves formation of blebs, in some cases clearly discernible as nuclear evaginations which detach (similar to pathway 5 in Fig. 34) and come to lie, still surrounded by pore-containing nuclear envelope, more caudad into the middle piece (Bröckelmann, 1963; Werner, 1966; deKretser, 1969; Rattner and Brinkley, 1971; Stanley, 1971a). Thus, these translocated nuclear envelope blebs provide a good example of a chromatin-free nuclear envelope. Somewhat comparable to this special formation of isolated "empty" nuclear blebs might be the "nuclear remainder" in coccid sperm formation (Moses and Wilson, 1970) and the "accessory nuclei" occuring in the hymenopteran oogenesis (for details see King and Fordy, 1970).

Another group of nuclear membrane formations such as membranous whorls, tubulizations, myelin configurations, and localized inflations of the perinuclear cisterna are, in most cases, suspected to be the result of either an insufficient fixation or of a cytopathological anomaly. Such structures have been, however, extensively reported in the literature (reviews: David, 1964 and Blackburn, 1971; see also Meek and Moses, 1961; Carr, 1967; Adams and Hertig, 1969; Falk, 1969; Scharrer and Wurzelmann, 1969b; Flickinger, 1970; Ruby and Webster, 1972; Kilarski

Fig. 36 Examples of associations of the nuclear envelope with microtubules (a-c) and microfilaments (d). In a, note the attachment of the axial microtubular bundle of the intranuclear mitotic apparatus in the fungus, Phycomyces blakesleeanus, at an electron-dense plaque apposed to the inner nuclear membrane (arrows); b gives one example for a cytoplasmic microtubule (arrow) parallel with the nuclear envelope (NE) in an oocyte of the snail, Helix pomatia. The cross section in c shows individual (left arrow) and grouped (right arrow) microtubules within the macronucleus of the ciliate, Tetrahymena pyriformis. Some of these microtubules appear to be linked to the inner nuclear membrane (left arrowhead) as they are linked to each other by lateral cross-bridges (right arrowhead). Note an abundance of perinuclear microfilaments in a HeLa cell (d) which are intimately associated with the nuclear envelope (NE). N, nucleus; C, cytoplasm, No, nucleolus; NP, nuclear pore complex $(a, \times 72,000 ; b, \times 75,000 ; c, \times 120,000 ; d, \times 56,000$; the bars in a, b, and $\mathrm{d}$ indicate $0.5 \mu \mathrm{m}$, that in c presents $0.1 \mu \mathrm{m}$ ). 
and Jasinski, 1970). Some of them have also been discussed in relation to developmental stages of cytoplasmic organelles as well as for nucleocytoplasmic transport and intracisternal storage.

Formation of paired cisternae at the nuclear envelope is a widely reported phenomenon, and can be especially dramatic in cell stages in which degeneration of ER occurs as, for instance, in sieve tube development in plants (Esau and Gill, 1971). Paired cisternae might result from a zippering up of ER-fragments alongside the nuclear surface, thus resulting in twin or even multiple cisternal stack associations (see also Rattner and Brinkley, 1971). The interspace between the two parallel membranes is filled rather homogeneously with ill-defined, fuzzy material or is bridged by threadlike connective elements (Franke et al., 1971c). There is further a marked tendency of isolated cisternal pieces to appose each other, as is illustrated by the observations that during nuclear envelope breakdown the cisternal fragments can become integrated into such paired cisternae with a high frequency (Barer et al., 1961; Murray et al., 1965; Hanaoka and Friedman, 1970; for further references see Szollosi et al., 1972a). Paired cisternae, however, are not always such "stacked remnants of the nuclear envelope" which are not reutilized for nuclear envelope reconstitution. This has been concluded by Kelley (1972), who found that they disappear in the presence of effective, sublethal doses of actinomycin D and puromycin, indicating that their normal origin depends on protein synthesis and takes place during interphase rather than in mitosis. Another argument is the inducibility of such cisternal stacking specifically at the nuclear surface. For instance, deuteron irradiation of mouse cerebellum leads to the envelopment of the nucleus with sometimes more than fifteen tightly appressed cisternae (Samorajski et al., 1968).

Local differentiations of the nuclear envelope into regions lacking pores or with a particularly high pore frequency have been mentioned in the discussion of pore complex patterns (Section VII).

Long sheets or tubular evaginations limited by the nuclear envelope as well as isthmi connecting the nuclear subdivisions in highly lobated nuclei are characteristic of special cells and have been described in a variety of cell types, most impressively in mammalian leukocytes, leukemic stages included, and in a series of tumor cells (Sebuwufu, 1966; Huhn, 1967; Davies and Small, 1968; Smith and O'Hara, 1968; Mollo et al., 1969; Haynes and Davies, 1973; see these articles for further reports). The common feature of such narrow nuclear extensions is their dense content, i.e., they can be regarded as nuclear outpocketings containing nothing but the nuclear envelope-attached chromatin.

A unique formation of regularly arranged short "hollow" cones 
(macrotubules) on the outer nuclear membrane has been described in the "wings" of the spermatid nuclei of ostracods (Reger and Florendo, 1969; Zissler, 1969.

\section{Associations and Interactions of the Nuclear Envelope with Microtubules and Microfilaments}

Microtubules are in various cells conspicuously accumulated in the immediate vicinity of the nucleus. Such juxtanuclear microtubule aggregations are not limited to the area surrounding centrioles and centrioleequivalent structures (MTOC, Pickett-Heaps, 1969, 1971; Manton et al., 1969a, b). Nor are they confined to late stages of mitotic and meiotic prophases in which the microtubules often exhibit a preference for orientations perpendicular to the nuclear envelope (see Section XII). They are also observed in interphase or earlier mitotic and meiotic prophase stages in which the tubules mostly abut the nuclear surface tangentially (Fig. 36b). Although in many situations such associations could be fortuitous and without any functional meaning, there are impressive demonstrations of regular arrays of microtubules closely paralleling the nuclear envelope (for synopsis of the literature see Franke, 1971a). The most prominent ones have been described in plant and animal spermatids (Kessel, 1966; Anderson, 1967; Paolillo et al., 1968; Fawcett, 1958, 1970; Fawcett et al., 1971; Rattner, 1972) during sporogenesis of the horsetail, Equisetum limosum (Manton, 1964; Sakai, 1968), and the moss, Mnium hornum (Lambert, 1970), and in the SV5 virus-infected hamster kidney cells described in the study of Holmes and Choppin (1968). This association is often seen to be stabilized by lateral linkages with the outer nuclear membrane (Kessel, 1966, 1970; Anderson et al., 1967; Bajer and Molè-Bajer, 1969; Fawcett et al., 1971; see also Burgess, 1970, and Phillips, 1970), in a manner similar to the cross-bridges observed in other microtubule-membranes (Pickett-Heaps and Northcote, 1966; Cronshaw, 1967; Kiermayer, 1968; Hepler et al., 1970; Olson and Kochert, 1970; Roth et al., 1970; Smith, 1970; Fawcett et al., 1971; Franke, 1971b, c; Friedman, 1971; Fuge, 1971; Yamada et al., 1971; LaFountain, 1972) and microtubule-microtubule associations as well (Grimstone and Cleveland, 1965; McIntosh and Porter, 1967; McIntosh et al., 1969; Roth et al., 1970; Brown and Franke, 1971; Tilney, 1971). Terminal anchoring of microtubules at the outer nuclear membrane has also been repeatedly described (Anderson, 1967; for prophase situations see: Bajer and Molè-Bajer, 1969, 1972; Sakai, 1969a, b). 
As for the function of such outer nuclear membrane-microtubule interactions three hypotheses have been pursued:

(a) A functioning as a nuclear exoskeleton, i.e., in the maintenance of the special nuclear morphology and in providing the nucleus with a cytoplasmic shell of increased rigidity. Such a role would be compatible with most of the current concepts on the general functioning of microtubules (review in Tilney, 1971). In fact, there are further observations which seem to support this view. For instance, Roberts and Northcote (1971) have noted that microtubules often run into the cytoplasmic indentations of the nucleus (the "tunnels"). A similar microtubule-filled cytoplasmic tunnel is characteristically found in the division of the dinoflagellate nucleus (Leadbeater and Dodge, 1967; Kubai and Ris, 1969). Woodcock (1971) has observed an abundance of perinuclear microtubules at the secondary nuclei of Acetabularia and postulated that they serve as connections between nuclear envelope and plasma membrane. A contribution of juxtanuclear microtubules to the stability of the various forms of nuclear extensions (see Section IX) has also been discussed (Bessis and Breton-Gorius, 1967; Norberg, 1969; Haynes and Davies, 1973).

(b) The idea that the microtubules surrounding the nuclear envelope play a major role in establishing the characteristic form of nonspheroidal nuclei, especially elongated ones, has come from studies of spermiogenesis (McIntosh and Porter, 1967; Kessel, 1970; Duckett, 1973) and nuclear "sheets" and lobes (Bessis and Breton-Gorius, 1967; Norberg, 1969; Haynes and Davies, 1973). An involvement of the nuclear envelope-microtubule association in nuclear-shaping processes is also suggestive in the late prophase in the green alga Oedogonium, where the polar parts of the envelope appear to be drawn out into a narrow cone by the attached microtubular sheath (Pickett-Heaps and Fowke, 1969), in the elongating nuclei of the blastoderm stage of Drosophila (Fullilove and Jacobson, 1971), and from the "pushing and pulling" of the cytoplasmic microtubules at the prophase nuclear envelope (Bajer and Molè-Bajer, $1969,1972)$. Likewise, such microtubules appear to be involved in the initiation of nuclear papilla formation in the spermiogenesis of the coccid Steatococcus where the basal parts of the growing microtubules appear to be laterally connected with the nuclear envelope cone (Moses and Wilson, 1970). In a recent review, however, three experts in spermiogenesis have examined the evidences present and arrived at the conclusion "that the form of the sperm head is probably not a consequence of external modeling by pressures applied to the condensing spermatid nucleus by microtubules in the surrounding cytoplasm . . ." (Fawcett et al., 1971). This conclusion was based on various observations such 
as $(i)$ that in mammalian sperm the manchette is confined to the caudal part of the nucleus only, ( $i i)$ that at maximal rate of nuclear elongation in early avian sperm head morphogenesis microtubules can be already longitudinally oriented, and (iii) that neither in mammals nor in all birds do the microtubules come in close contact with the outer nuclear membrane but can be separated from it by a ca. $600 \AA$ broad coat of fuzzy, fibrillar material. At the moment the prevailing concept on nuclear shaping and morphology maintenance seems to be that the modeling forces are exerted by changes of the chromatin itself (Fawcett et al., 1971; Lanzavecchia and Donin, 1972).

(c) Bundles of cytoplasmic microtubules which are parallel to or terminally attached to the nuclear envelope seem to participate in the nuclear division processes (intranuclear chromosome segregations) of dinoflagellates (Leadbeater and Dodge, 1967; Kubai and Ris, 1969; Soyer, 1969c, 1971) and holomastigid flagellates (Hollande and Valentin, 1968a, b; Hollande and Carruette-Valentin, 1971), in a mode suggesting an action as an extranuclear spindle apparatus.

(d) An involvement of the juxtanuclear microtubules in nuclear locomotion or in movements of cytoplasmic components relative to the nuclear surface has been indicated in the study of Holmes and Choppin (1968; see also Aronson, 1971). It is, however, quite conceivable that these microtubules are not involved in the generation of the force for such intracellular movements but rather serve as "guide elements" along which other cell components, including nuclei, slide (Holmes and Choppin, 1968).

(e) Such microtubular accumulations might represent juxtanuclear tubulin storages which could be used for processes in nuclear division or morphogenesis or function as membrane-associated MTOC (PickettHeaps, 1969; see the above quoted references, especially Manton et al., 1969a, b).

The inner nuclear membrane has also been demonstrated to be associated with various types of intranuclear microtubule formations. The terminal insertion of the axial bundles in various algal, fungal, and protozoan mitoses has already been mentioned in Section IX. Terminal attachment of intranuclear microtubules to the envelope has also been reported for prophases of plasmodial nuclei of the slime mold, Physarum polycephalum (Blessing, 1972), and for micro- and macronuclei of various ciliates (Jenkins, 1967; Falk et al., 1968; Ito et al., 1968; Wunderlich and Speth, 1970; Raikov, 1973). In the micronuclei of the ciliate Nassula, however, the spindle tubules appear to terminate at a special flattened intranuclear vesicle (Tucker, 1967). In addition, it has been shown that intranuclear microtubules can abut the inner nuclear membrane at a 
rather low angle and frequently are parallel to it, thereby sometimes revealing lateral cross-bridge connections to the membrane surface (Fig. 36c; Tucker, 1967; Wilson, 1969; Tamura et al., 1969; Jurand and Selman, 1970; Franke, 1971d; see also Pickett-Heaps and Fowke, 1969). One possibility is that such microtubules function as an intranuclear corset and contribute to nuclear shaping, or are involved in intranuclear movement processes such as chromosome segregation. Nuclear shaping is strongly suggested in those mitoses (in several fungi, algae, and ciliates) where the growing intranuclear axial bundle elongates and seems to push its nuclear envelope insertion sites apart, thus separating the nuclear envelope into halves. In some cases this leaves a cylinder-shaped nuclear envelope remainder that is not included in the two daughter nuclear envelopes (the "separation spindle" of the early literature; Jenkins, 1967; Moor, 1967; Tucker, 1967; Ichida and Fuller, 1968; Raikov, 1968; Jurand and Selman, 1970; Stevenson and Lloyd, 1971; Ott and Brown, 1973).

As in the prometaphase of "open" and "polar fenestrae" type mitoses (see Section XII) it is a frequent and intriguing observation that the intimate associations of the nuclear membranes with microtubules and fibrillar aggregates are spatially and temporally correlated with alterations of membrane structure (of the kind described in Section IX) or with membrane breakdown (Section XII; for review see Franke, 1971a; compare also Moses and Wilson, 1970; Yasuzumi et al., 1971).

Tangles of filaments (with widths in the range from 40 to $120 \AA$ ) at the nuclear envelope have also been observed in diverse cell types, most conspicuously as tufts on the outer nuclear membrane (Fig. 36d; reviewed by Franke, 1971a). As with the envelope-associated microtubules, an involvement of such fibrillar aggregates in perinuclear streaming and other intracellular movement phenomena has been hypothesized. On the other hand, there are indications that an attachment of the nuclear envelope to such filamentous structures is simply a means of connecting the nucleus to other cell components (Du Praw, 1965; Franke, 1971a). This is suggested from $(i)$ the insertion of a flagellar rhizoplasts at the nuclear envelope (e.g., Joyon, 1963; Mignot, 1967; Pitelka, 1969; Hibberd, 1970; Franke, 1970c; Slankins and Gibbs, 1972; Watson and Arnott, 1973; Bouck and Brown, 1973); (ii) from the sometimes regularly spaced filamentous connections between the outer nuclear membrane and the aggregates associated with centriolar bases and connecting pieces (Fig. 37; Fawcett and Phillips, 1970; Fawcett, 1972; Picheral, 1972b); and (iii) from the association with myofilaments. The latter have been thought to provide a means for translating the cellular contraction to the nucleus, resulting in the characteristic concertinalike 


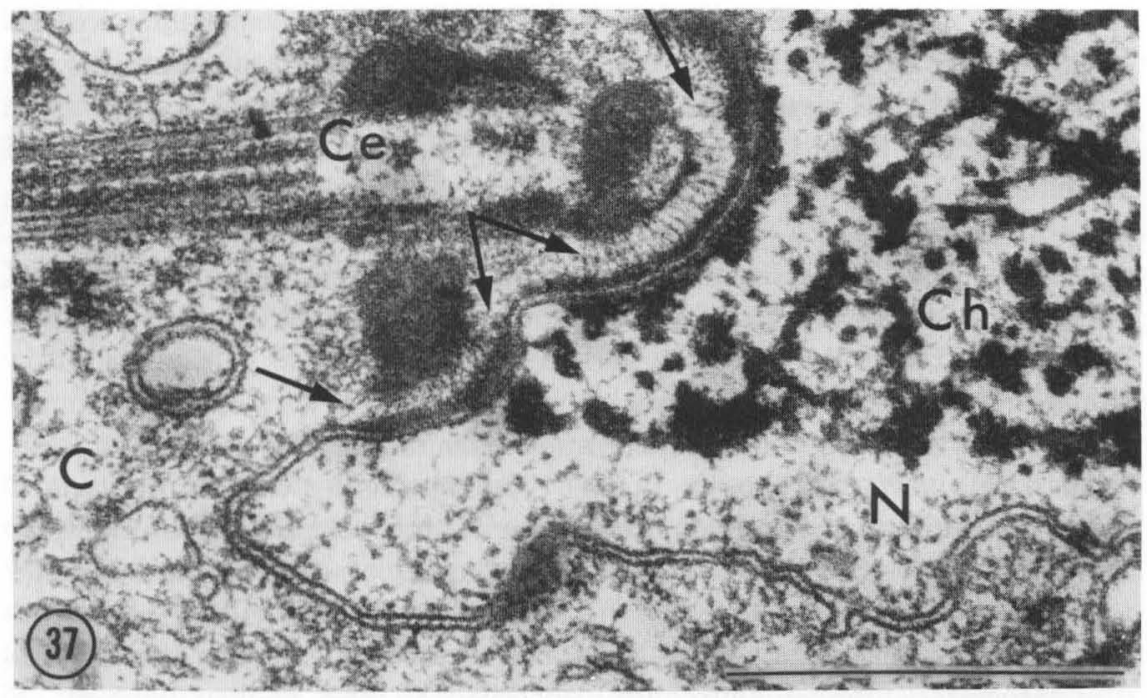

Fig. 37 Filamentous connections between the outer nuclear membrane-apposed layer and the dense aggregate bodies associated with the centriole ( $\mathrm{Ce}$ ) basis in a rat spermatid (arrows). Such filaments can be regularly spaced (as seen in the region between the upper two arrows). Note also the altered appearance of the perinuclear cisterna in these regions. $\mathrm{N}$, nucleus; $\mathrm{Ch}$, chromatin; $\mathrm{C}$, cytoplasm $(\times 80,000$, bar indicates $0.5 \mu \mathrm{m})$.

folding of the nuclear envelope in contracted muscle cells (Bloom and Cancilla, 1969; Franke and Schinko, 1969; Franke, 1970d). However, in a great many cell types one should also think of possible "cytopathological" origins of such structures, for instance, as assemblies of material which has locally accumulated after disintegration of other cell components such as ribosomes and/or membranes (Franke, 1971a; see also Daniels et al., 1968).

\section{Relationship of the Nuclear Envelope to Other Cell Organelles}

It has already been mentioned that the nuclear surface is, in many Chromophyta, coupled with the plastids by a common surrounding cisterna (Section IV). A similar connection has hitherto not been reported for mitochondria. Nevertheless, mitochondria, in a diversity of cell types, accumulate at the nuclear surface and appear somehow attached to it (e.g. Ornstein, 1956; Drawert and Mix, 1961; Balinsky and 
Devis, 1963; Meyer, 1963; Baker and Franchi, 1969; Hsu, 1967; Kessel, 1968b; Aikawa et al., 1970; Rowley et al., 1971; for further references see Franke, 1974), as demonstrable by the tenacity with which they stick to isolated nuclei and even to the envelopes. Such an interaction might be effected simply by hydrophobic surface adsorption, but structures possibly involved in a direct fixation of such nuclear surface-mitochondria associations are also noticeable. In particular, this type of connection appears to involve threadlike bridges between the outer nuclear membrane and the mitochondrial surface (Franke et al., 1973a) and is sometimes suggestive of an involvement of nuclear envelope-ribosomes (Fig. 21f). Accumulation of juxtanuclear mitochondria appears also in association with the "heavy body" type aggregates which in various cells accumulate at the nuclear surface and probably are identical to the nucleocytoplasmically transported particulate clumps described in Section VII (Ornstein, 1956; the ciment intermitochondrial of Clerot, 1968; for further references see Section VII). A similar type of mitochondria-associated "dense aggregate" observed in the nuclear vicinity is the already mentioned "chromatoid body" formed during spermiogenesis (reviews: Fawcett, 1972; Comings and Okada, 1972b; Schjeide et al., 1972). It is not known whether either the juxtanuclear densely stained aggregates or the juxtanuclear mitochondria have something to do with a specific nuclear function or with the regulation of nucleocytoplasmic exchange processes. The close association of mitochondria with the nuclear envelope has repeatedly stimulated cytologists to discuss a possible de novo formation of mitochondria from nuclear envelope blebs or evaginations (Brandt and Pappas, 1959; David, 1964; for further references see the recent articles by Stang-Voss and Staubesand, 1970, and Bell, 1972); this hypothesis is neither convincingly supported by the micrographs presented nor is likely on cytogenetic grounds.

A "classic" association with the nuclear envelope is that of the centrioles or centriolar equivalents. They may occur, as usual, singly or as pairs or in multiples (recent reviews: Fulton, 1971; Wolfe, 1972; for examples of the latter type see Szollosi et al., 1972b, and PickettHeaps, 1971; higher plant cells which have been widely regarded as not possessing centriolelike structures do, however, have spindle poledetermining aggregates of small vesicles and microtubular pieces in the perinuclear cytoplasm, Esau and Gill, 1969). The centriolar structures are often located in defined pockets of the nuclear envelope, basally associated with ill-defined dense aggregates and the centriole adjuncts, and sometimes reveal electron-opaque continuities with the outer nuclear membrane (Fig. 37; Fawcett and Phillips, 1970; Picheral, 1972b; Zentgraf and Franke, 1974). It appears that the centriole is truly, though perhaps 
loosely, connected to the envelope membranes proper. De novo formation of centrioles in close association with the nuclear envelope is also suggested in the micrographs of various authors (see Outka and Kluss, 1967; Swale, 1969; Fawcett, 1972; Wolfe, 1972).

\section{Breakdowns and Reconstitutions of the Nuclear Envelope; Role of the Nuclear Envelope in Nuclear Divisions and Fusions}

Breakdown of the nuclear envelope is known to occur in certain stages of cell degeneration and in other cases of cytopathology and is mostly observed as an irregular disintegration of the perinuclear cisterna into vesicles and cisternal fragments. This is frequently accompanied by a series of other phenomena such as increase in osmophilia, especially at the inner nuclear membrane, extensive invagination and vesiculation, and a tendency to form "paired cisternae" arrangements (see Section IX; the relevant literature has been excellently reviewed by David, 1964, and Blackburn, 1971). A particularly well-studied example is the nuclear degeneration which takes place during phloem differentiation in the sieve elements of various plants (Esau and Gill, 1971; Evert and Deshpande, 1970; Esau, 1972). Here one sees that even the very early stages of nuclear envelope disintegration result in an invasion of the nucleoplasmic space by the cytoplasmic ribosomes, i.e., the breakdown of the characteristic barrier function for particle exchange (see also Brachet et al., 1970). The nuclear envelope disintegration is also often accompanied by the appearance of microfilaments originating from the nuclear envelope.

The regular and progressive nuclear envelope breakdowns in many sperm cells can be either gradual (as in various spermiogeneses; for references see Section II) or rather rapid (as within the fertilized egg). In both situations it is not known what induces the membrane disintegration.

The nuclear envelope breakdown which occurs during "nuclear fragmentation" in the extracellular microgametocytes of the avian leukocyte parasite, Leucocytozoon simondi, has been described in detail by Aikawa et al. (1970). Here the nuclear envelope fragments contain pore complexes and remain partially associated with the free chromatin clumps.

A programed breakdown takes place in mitotic prometaphase and meiotic diakinesis in many, though not all, organisms. One might catalogue the various mitotic forms in an order of an increasing extent of nuclear envelope disintegration which, according to Pickett-Heaps 
(1969), suggests something like an "evolutionary line" of mitotic morphology. As already mentioned (Section IX) what perhaps represents a very limited and localized nuclear envelope transition and disintegration is found in the polar thickenings at the inner nuclear membrane and the polar plaques in various algae, fungi (with the exception of the Basidiomycetes), and protozoa. One has also to add to this the microtubule attachment sites at the kinetochore-fixing regions of the nuclear envelope of some hypermastigid flagellates (Cleveland, 1938, 1957a, b; Hollande and Valentin, 1968a, b). A further step in nuclear envelope disintegration is exhibited by those mitoses in which most of the nuclear envelope persists throughout mitosis, except for the "polar fenestrae" through which the spindle microtubules seem to invade the nuclear region. This form is especially widespread among the algae (Johnson and Porter, 1968; Marchant and Pickett-Heaps, 1970; Løvlie and Bråten, 1970; Pickett-Heaps, 1970, 1972; McDonald, 1972; Neushul and Dahl, 1972) but occurs also in other organisms, for instance in slime mold plasmodia (Guttes et al., 1968; Aldrich, 1969; Ryser, 1970) and in Ascaris spermatocytes (Favard, 1961). A next step in progressive nuclear envelope disintegration seems to be located preferentially in the equatorial region (Løvlie and Brăten, 1970). Partial dispersal is frequently recognized, though not always at the spindles poles, in Basidiomycetes (McCully and Robinow, 1972a, e.g., observed a "large gap on one side" in the nucleus of Leucosporidium scottii) and in giant amoebae (Roth et al., 1960). A peculiar pattern of nuclear envelope disintegration has been sketched for the heterobasidiomycetous yeast, Rhodosporidium, by McCully and Robinow (1972b), who noted a disruption at one side only and, later, that "the spindle-containing [nuclear] portion pinches off from the rest of the nucleus."

The final stage characterized by complete prometaphase disruption of the envelope is the classic "open" nuclear division (Pickett-Heaps, 1969). Here the perinuclear cisterna disintegrates, again mostly starting in the polar areas, into cisternal pieces or small vesicles which may spread out, and hence are indistinguishable from ER elements, or remain attached to the chromosomal surfaces up to anaphase (David, 1959, 1964; Moses, 1960; Porter and Machado, 1960; Chang and Gibley, 1968; Esau and Gill, 1969; Sprey and Hasche, 1972; for review see Bajer and Molè-Bajer, 1972).

The first visible alteration indicating the initiation of nuclear envelope breakdown in mitosis and meiosis is a conspicuous tortuosity of the nuclear envelope ("undulation"; Thomas, 1964; Bajer and Molè-Bajer, 1969, 1972; Brachet et al., 1970; Calarco et al., 1972; Gondos et al., 1972; Szollosi et al., 1972a, b). In some meioses this is accompanied 
by a reduction of pore complexes. In many mitotic and meiotic divisions this stage is further characterized by the appearance of a perinuclear "clear zone" in which microtubules accumulate (for references see Burgess, 1970; Luykx, 1970; Bajer and Molè-Bajer, 1972).

It is interesting to note that normal pore complexes are in some cases identifiable during chromosome distribution on the cisternal fragments of the old or the new (reconstituting) nuclear envelope (Moses, 1960; Harris, 1961; Stevens, 1965; Kessel, 1966; Bajer and Molè-Bajer, 1969; Schwalm, 1969; Burgess, 1970; Roberts and Northcote, 1971; Sprey and Hasche, 1972), in addition to being normally present in the persisting nuclear envelope parts of the "polar fenestrae" mitoses (Aldrich, 1969; Guttes et al., 1968; Pickett-Heaps and Fowke, 1969; Ryser, 1970; Neushul and Dahl, 1972; for changes in pore complex appearance see, however, Johnson and Porter, 1968). This again emphasizes that pore complexes are structures independent of both the continuation of transcription and nucleocytoplasmic compartmentalization and transport (see Section VII). Furthermore, there are some electron micrographs which suggest that, upon the disruption of the nuclear envelope, ribosomes can become attached to the inner surface of the perinuclear cisterna fragments. This demonstrates that the characteristic interphase polarity of the envelope with respect to the attachment of ribosomes and chromatin is not endogeneous to the membrane leaflet itself but rather reflects the exclusion of ribosomes from the interphase nucleoplasm (Esau and Gill, 1969; Pickett-Heaps, 1970; Roberts and Northcote, 1971).

Microtubules penetrate into the region of the forming spindle through the first "gaps" in the nuclear envelope, either as bundles or as isolated tubules which seem to preferentially "pierce" the envelope perpendicularly to the surface (Pickett-Heaps and Northcote, 1966; Bajer and Molè-Bajer, 1969, 1972; Esau and Gill, 1969; Manton et al., 1969a, b; Fowke and Pickett-Heaps, 1969; Pickett-Heaps and Fowke, 1969; Sakai, 1969a, b; Marchant and Pickett-Heaps, 1970; Tanaka, 1970; Bech-Hansen and Fowke, 1972; Calarco et al., 1972; McDonald, 1972; Pickett-Heaps, 1972; Slankis and Gibbs, 172; Szollosi et al., 1972b). They do not run through the pore complexes as has been earlier suggested by Ledbetter (1967).

A strictly vectorial progress of nuclear envelope disintegration, beginning at the basal (vegetative) pole, has been demonstrated by Brachet and his associates (Brachet $e t$ al., 1970) with the germinal vesicle maturation (in vitro) in Xenopus laevis oocytes.

A special feature of some mitotic forms is the appearance of an additional sheath which surrounds most of the nuclear region after nuclear envelope breakdown, thus providing something like a perinuclear 
envelope of ER that seems to shield the mitotic configuration from penetration of large cytoplasmic organelles (Marchant and Pickett-Heaps, 1970; Pickett-Heaps, 1970, 1972; McDonald, 1972).

Neither the molecular mechanism nor the biological function of the nuclear envelope breakdown is understood. That nuclear breakdown is not an obligatory prerequisite for an ordered chromosome distribution is best illustrated by the various forms of intranuclear mitosis and meiosis (for the latter see also Howard and Moore, 1970). Transplantation experiments with amoeba nuclei (Feldherr, 1968b) have shown that, in this cell system, during mitosis "the cytoplasm contains no factor capable of disrupting the nuclear envelope." Hancock and Ryser (1967) have developed the concept that basic proteins which become liberated during mitotic prophase might act as membranolytic agents, an idea that is based on the known effects of positively charged polymers on other membranes (for review see Ryser et al., 1971). Subsequent experiments, however, have shown that no considerable amounts of histones, the suggested candidates for this action, are released from the chromatin (Hancock, 1969). Therefore, the histones are, at least at the moment, regarded as unlikely to be the inducers of nuclear membrane disintegration. Alternatively, Bajer and Molè-Bajer (1969) have suggested that the mechanical action of the prophase microtubules contributes to the disruption of the nuclear envelope. An especially advantageous experimental system to study the mechanisms of nuclear envelope breakdown is certainly the hormone-inducible in vitro breakage of the amphibian germinal vesicle (see the above quoted work of Brachet et al., 1970).

The nuclear envelope plays an important role in the intranuclear mitoses and meioses (for the latter see Howard and Moore, 1970). Morphological studies suggest that in these processes the nuclear envelope contains special sites which serve as seeds or nucleating centers which control microtubular growth and orientation (for reviews see PickettHeaps, 1969, and Franke, 1974; see further Wilson, 1970). Further, the envelope as a whole represents a structure which assists, together with the elongating microtubules, in nuclear fission. In addition, the envelopes of some nuclei contain attachment sites of chromosomes (the best example for this is provided by Hypermastigotes and dinoflagellates) which are distributed to the daughter nuclei as a remaining in their attachment to the nuclear envelope halves (Grassé, 1952; Cleveland, 1957a, b; Hollande and Valentin, 1968a, b; Hollande and Carruette-Valentin, 1971). Membrane loci may contribute to both the kinetochores and the polar structures in open and polar fenestrae mitoses (see also Section V) so that spindle formation is induced by the liberation of such sites and their exposition to the monomer pool. Therefore, following the hypothe- 
sis of Pickett-Heaps (1969) of membrane-associated MTOC, it appears reasonable to examine the role of the nuclear envelope, or components thereof, as an integral part of the chromosome distribution apparatus, in both open and closed nuclear divisions.

The reconstitution of the nuclear envelope in anaphase-telophase begins as an apposition of cisternal pieces and/or vesicles on the surface of the individual chromosomes (Amano and Tanaka, 1957). There have been only a few cases reported where a new envelope is formed within the old one, namely, in the intranuclear mitosis of the micronuclei of some ciliates (Jenkins, 1967; Inaba and Sotokawa, 1968; Suganuma, 1969; Raikov, 1973). This again illustrates that, at least in special cases, membranes can form (assemble) de novo in the nuclear interior, as has already been discussed in connection with the appearance of intranuclear cisternae (Section VIII) and is also indicated in the virion envelopment processes of some nuclear polyhedrosis viruses (Stoltz et al., 1973). With the "open" mitoses, it is still not clear whether ER elements or remainders of the mother nuclear envelope or both are used in the construction of the new envelope, or whether a de novo synthesis of the nuclear envelope takes place. In any case, the organizing structure is the surface of the individual chromosome, perhaps functioning as "seeds" for membrane assembly. In various cell systems envelopes are first completed around the individual chromosomes, i.e., karyomeres are formed. Subsequent fusion of these karyomeres in a second process results in the completed nucleus, i.e., a common nuclear envelope for all chromosomes (Barer et al., 1959, 1960; Moses, 1960; Harris, 1961; Harris and Mazia, 1962; Stevens, 1965; Schwalm, 1969; Sachs and Anderson, '1970). Reconstitution of the nuclear envelope is often initiated at the "leading edges" of the chromosomes, i.e., in the pericentromeric region (Robbins et al., 1964; Thomas, 1964). On the other hand, one also sometimes observes that a polar gap remains the last part of the new envelope to be formed. Again pore complexes are often recognized before the whole envelope is reconstituted (Harris, 1961).

A controlled sequence of membrane disintegration is also observed in processes of nuclear fusion (Jensen, 1964; Crawley, 1966; Brown et al., 1968; Urban, 1969). Usually, the outer nuclear membranes of the two mating nuclei begin to fuse, where they are opposed, by local membrane coalescence at one or several "bridging" sites, thus giving rise to a common perinuclear cisterna. The inner membrane in this region then also dissolves and the nuclear contents merge to complete the karyogamy. In some cytological situations nuclear fusion is concomitant with the total vesiculation of the entire nuclear envelope, and is directly transient into the prometaphase of the first cleavage mitosis (Longo 
and Anderson, 1968, 1969; see there for earlier references). Nothing is known yet as to what causes the nuclei of different or equal character to fuse. From the morphological studies one might suggest that both determinants, recognition and membrane fusion, are properties of the specific nuclear membranes themselves.

\section{Note Added in Proof}

Since the completion of this manuscript three more reviews on nuclear membrane structure and biochemistry, and isolation techniques have appeared [Berezney, R. (1973) Methods in Cell Physiol. 8, in press; Kay, R. R., and Johnston, I. R. (1973) Sub-Cell. Biochem. 2, 127; Kessel, R. G. (1973) In "Recent Progress in Surface and Membrane Science" (J. F. Danielli, A. E. Riddiford, and M. D. Rosenberg, eds.) Vol. 6, pp. 243-329, Academic Press, New York], in addition to a large number of original research communications. The majority of these articles has been dedicated to the question of the attachment of DNA to the nuclear membrane and the possible dynamic functions of this relationship [Fujiwara, Y. (1972) Cancer Res. 32, 2089; Aronson, J. F. (1973) J. Cell Biol. 58, 126; Barrieux, A., Long, G. L., and Garren, L. D. (1973) Biochim. Biophys. Acta 321, 228; Comings, D. E., and Okada, T. A. (1973) J. Mol. Biol. 75, 609; Freienstein, C. M., Freitag, H., and Süss, R. (1973) FEBS Letters 30, 170; Huberman, J. A., Tsai, A., and Deich, R. A. (1973) Nature (London) 241, 32; Infante, A. A., Nauta, R., Gilbert, S., Hobart, P., and Firshein, W. (1973) Nature New Biol. 242, 5; Oppenheim, A., and Wahrman, J. (1973) Exp. Cell Res. 79, 287; Wise, G. E., and Prescott, D. M. (1973) Proc. Nat. Acad. Sci. U.S. 70, 714; Yamada, M., and Hanaoka, F. (1973) Nature New Biol. 243, 227]. Among these articles, however, there is an increasing number in which it is concluded that replication sites are neither in early $S$ phase nor at any other time of $\mathrm{S}$ phase exclusively or preferentially located at the inner nuclear membrane. In a study on "particle" distribution in freeze-fractured pneumocyte nuclear envelopes, Meyer et al. [Meyer, H. W., Roth, J., and Bolck, F. (1972) Protoplasma 75, 313] noted a delicate particle pattern, which they discussed with respect to a hypothetical mode of membrane growth, and particle-free areas which they speculated might represent attachment plaques of chromosomes. A report on the presence of defined "cell surface antigens," the H-2 histocompatibility antigens in the mouse, in thymus and liver nuclear membranes, has also appeared [Albert, W. H. W., and Davies, D. A. L. (1973) Immunol. 24, 841]. Novel structures in association with the nuclear envelope are the lamella aggregates which appear during prophase of 
the intranuclear mitosis of the micronucleus of the ciliate, Ichthyophtirius multifiliis [Hauser, M., and van Eys, H. (1973) Cytobiol. $7,215]$. One of the most careful studies of an intranuclear chromosome distribution mechanism has been presented by D. Kubai [Kubai, D. F. (1973) J. Cell Sci. 13, 511], who described the centromeric and microtubular attachment discs in the nuclear envelope of the flagellate, Trichonympha agilis. A cell physiological study on the factors controlling pronuclear fusion in the sea urchin, Lytechinus variegatus, arrived at the conclusion that "the potential for nuclear fusion is not necessarily related to the cell cycle and that modification of the nuclear envelope . . restricts nuclear fusion" [Aronson, J. F. (1973) J. Cell Biol. 58, 126]. C. M. Feldherr [(1973) Experientia 29, 546] studied the effects of temperature on the nuclear permeability for gold particles of various sizes (25-170 $\AA$ in diameter) after cytoplasmic injection into amoeba. As far as the biochemical characterization is concerned, negative results with binding of colloidal iron hydroxide [Virtanen, I., Nordling, S., and Wartiovaara, J. (1973) J. Ultrastruct. Res. 42, 400) have been reported besides positive findings with respect to the presence of NAD pyrophosphorylase [Green, S., and Mamaril, F. P. (1973) Biochem. Soc. Transact. $1,636]$ and of a variety of membrane-bound enzymes in a plant system (following reference). There have also been new descriptions of fractionation of nuclear membranes from pea plumules [Stavy, R., BenShaul, Y., and Galun, E. (1973) Biochim. Biophys. Acta 323, 167], from mammalian liver [with the use of the polyanion, heparin; Bornens, M. (1973) Nature (London) 244, 28], and avian late erythroblasts and erythrocytes (Harlow, R., Tolstoshev, P., and Wells, J. R. E. (1972) Cell Different. 2,341). An intensive cytochemical study on the formation of AL in oocytes of the polychaet worm Nereis [Dhainaut, A. (1973) Z. Zellforsch. 137, 481] has supported the RNP character of the pore complex material and also the idea that AL can form from preexisting ER cisternae.

\section{ACKNOWLEDGMENTS}

We thank our friends and colleagues for the discussions and stimulating criticisms as well as for their cooperation in the work on the nuclear envelope. We are particularly indebted to Drs. B. Deumling, W. Eckert, H. Falk, E. D. Jarasch, J. Stadler, and H. W. Zentgraf, as well as to Mr. M. Trendelenburg and J. Kartenbeck. We thank Miss $S$. Krien and M. Winter for careful technical assistance. We are also grateful to Drs. R. Kay (University College, London, England), T. W. Keenan (Purdue University, Lafayette, Indiana), and P. M. M. Rae (Yale University, New Haven, Connecticut) for reading, discussing, and correcting the manuscript. The project has received support from the Deutsche Forschungsgemeinschaft, Bad Godesberg, Germany. 


\section{REFERENCES}

Abelson, H. T., and Smith, G. H. (1970). J. Ultrastruct. Res. 30, 558.

Abelson, P. H., and Duryee, W. R. (1949). Biol. Bull. Woods Hole 96, 205.

Adams, E., and Hertig, A. T. (1969). J. Cell Biol. 41, 716.

Adesnik, M., Salditt, M., Thomas, W., and Darnell, J. E. (1972). J. Mol. Biol. $71,21$.

Afzelius, B. A. (1955). Exp. Cell Res. 8, 147.

Afzelius, B. A. (1963). J. Cell Biol. 19, 229.

Agutter, P. S. (1972). Biochim. Biophys. Acta 255, 397.

Aikawa, M., and Beaudoin, R. L. (1968). J. Cell Biol. 39, 749.

Aikawa, M., Huff, C. G., and Sprinz, H. (1967). J. Cell Biol. 34, 229.

Aikawa, M., Huff, C. G., and Strome, C. P. A. (1970). J. Ultrastruct. Res. 32, 43.

Aldrich, H. C. (1969). Amer. J. Bot. 56, 290.

Aldrich, H. C., and Vasil, I. K. (1970). J. Ultrastruct. Res. 32, 307.

Alfert, M., and Das, N. K. (1969). Proc. Nat. Acad. Sci. U.S. 63, 123.

Allen, E. R., and Cave, M. D. (1968). Z. Zellforsch. 92, 477.

Al-Mukhtar, K. A. K., and Webb, A. C. (1971). J. Embryol. Exp. Morphol. 26, 195.

Altmann, H.-W., and Pfeifer, U. (1969). Virchows Arch. Abt. B. Zellpathol. 2, 220.

Amano, S., and Tanaka, H. (1957). Acta Haem. Jap. 20, 319.

Anderson, E., and Beams, H. W. (1956). J. Biophys. Biochem. Cytol. Suppl. 2, 439.

Anderson, W. A. (1967). Mol. Gen. Genet: 99, 257.

Anderson, W. A., Weissmann, A., and Ellis, R. A. (1967). J. Cell Biol. 32, 11.

André, J., and Rouiller, C. (1956). Proc. Stockholm Conf. Electron Misrosc. (F, S. Sjöstrand and J Rhodin, eds), pp. 162-164. Almquist and Wiksells, Uppsala.

ApGwynn, I., Barton, R., and Jones, P. C. T. (197I). Z. Zellforsch. 112, 390.

Aronson, J. F. (1971). J. Cell Biol. 51, 579.

Arrighi, F. E., and Hsu, T. C. (1971). Cytogenetics 10, 81.

Avrameas, S. (1970). Int. Rev. Cytol. 27, 349.

Avrameas, S., and Bouteille, M. (1968). Exp. Cell Res. 53, 166.

Balbai, F., Tremblay, G., and Dumont, A. (1969). J. Ultrastruct, Res. 28, 125.

Bach, M. K. (1962). Proc. Nat. Acad. Sci. U.S. 48, 1031.

Bach, M. K., and Johnson, H. G. (1966). Nature (London) 209, 893.

Bahr, G. F., and Beermann, W. (1954). Exp. Cell Res. 6, 519.

Bajer, A., and Molè-Bajer, J. (1969). Chromosoma 27, 448.

Bajer, A., and Molè-Bajer, J. (1972). In "Spindle Dynamics and Chromosome Movements" (G. A. Bourne and J. F. Danielli, eds), Int. Rev. Cyitol Suppl. 3 pp. 1-271. Academic Press, New York.

Baker, T. G., and Franchi, L. L. (1969). Z. Zellforsch. 93, 45.

Bal, A. K., Jubinville, F., Cousineau, G. H., and Inoué, S. (1968). J. Ultrastruct. Res. 25, 15.

Balinsky, B. I., and Devis, R. J. (1963). Acta Embryol. Morphol. Exp., 6, 55.

Barer, R., Joseph, S., and Meek, G. A. (1959). Exp. Cell Res. 18, 179.

Barer, R., Joseph, S., and Meek, G. A. (1960). Proc. Roy. Soc. B 152, 353.

Barer, R., Joseph, S., and Meek, G. A. (1961). In "Electron Microscopy in Anatomy" (J. D. Boyd, F. R. Johnson, and J. D. Lever, eds) pp. 161-175. Arnold, London.

Barker, K. R., and Biesele, J. J. (1967). La Cellule 67, 91.

Barnes, B. G., and Davis, J. M. (1959). J. Ultrastruct. Res. 3, 131. 
Barr, M. L. (1959). Science 130, 679.

Barr, M. L. (1966). Int. Rev. Cytol. 19, 35.

Barton, A. D., Kisieleski, W. E., Wassermann, F., and Mackevicius, F. (1971).

Z. Zellforsch. 115, 299.

Baud, C. A. (1953). Acta Anat. 17, 113.

Baud, C. A. (1959). Exposés act. Biol. Cell. 5, 1.

Baud, C. A. (1965). In "Intracellular Membraneous Structure" (S. Seno and E. V. Cowdry, eds.) pp. 323-329. Jap. Soc. Cell Biol., Okayama.

Beale, G. H., Jurand, A., and Preer, J. R. (1969). J. Cell Sci. 5, 65.

Beams, H. W. (1948). J. Morphol. 83, 87.

Beams, H. W., and Kessel, R. G. (1968). Cancer Res. 28, 1944.

Beams, H. W., and Mueller, S. (1970). Z. Zellforsch. 108, 297.

Beams, H. W., and Sekhon, S. S. (1969). J. Ultrastruct. Res. 26, 296.

Beams, H. W., Tahmisian, T. N., Devine, R., and Anderson, E. (1957). Exp. Cell Res. 13, 200.

Beaulaton, J. (1968). Z. Zellforsch. 89, 453.

Bech-Hansen, C. W., and Fowke, L. C.( 1972). Can. J. Bot. 50, 1811.

Beckett, A., and Crawford, R. M. (1970). J. Gen. Microbiol. 63, 269.

Beermann, W. (1964). J. Exp. Zool. 157, 49.

Behnke, O. (1968). J. Ultrastruct. Res. 24, 412.

Behnke, O., and Moe, H. (1964). J. Cell Biol. 22, 633.

Bell, E. (1971). In "Control Mechanisms of Growth and Differentiation" (D. D.

Davies and M. Balls, eds.), Symp. Soc. Exp. Biol. 25, pp. 127-143. Cambridge Univ. Press, London and New York.

Bell, P. R. (1972). J. Cell Sci. 11, 739.

Bell, P. R., and Mühlethaler, K. (1964). J. Cell Biol. 20, 235.

Ben-Porat, T., and Kaplan, A. S. (1971). Virology 45, 252.

Ben-Porat, T., Stere, A., and Kaplan, A. S. (1962). Biochim. Biophys. Acta 61, 150.

Ben-Porat, T., Shimono, H., and Kaplan, A. S. (1969). Virology 37, 56.

Berendes, H. D., and DeBruyn, W. C. (1963). Z. Zellforsch. 59, 142.

Berezney, R., and Crane, F. L. (1971). Biochem. Biophys. Res. Commun. 43, 1017.

Berezney, R., and Crane, F. L. (1972). J. Biol. Chem. 247, 5562.

Berezney, R., Funk, L. K., and Crane, F. L. (1970). Biochim. Biophys. Acta 203, 531.

Berezney, R., Macaulay, L. K., and Crane, F. L. (1972). J. Biol. Chem. 247, 5549.

Bernhard, W. (1969). J. Ultrastruct. Res. 27, 250.

Bernhard, W., and Granboulan, N. (1963). Exp. Cell Res. Suppl. 9, 19.

Berg, W. (1932). Z. Mikrosk. Anat. Forsch. 28, 565.

Bessis, M., and Breton-Gorius, J. (1967). Nouv. Revue fr. Hémat. 7, 601.

Bianchi, N. O., Sweet, B. H., and Ayres, J. (1971). Exp. Cell Res. 69, 236.

Binggeli, M. F. (1959). J. Biophys. Biochem. Cytol. 5, 143.

Bisalputra, T., and Burton, H. (1969). J. Ultrastruct. Res. 29, 224.

Bisalputra, T., and Burton, H. (1970). J. Microsc. 9, 661.

Blackburn, W. R. (1971). In "Pathobiology Annual 1971" (H. L. Joachim. ed.) pp. I-31.

Blessing, J. (1972). Cytobiologie 6, 342.

Blondel, B. (1968). Exp. Cell Res. 53, 348.

Bloom, S. (1970). J. Cell Biol. 44, 218.

Bloom, S., and Cancilla, P. A. (1969). Circ. Res. 24, 189. 
Boloukhère, M. (1970). In "Biology of Acetabularia" (J. Brachet and S. Bonotto, eds.), pp. 145-175. Academic Press, New York.

Bond, H. E., Cooper, J. A., Courington, D. P., and Wood, J. S. (1969). Science 165, 705 .

Bornens, M. (1968). C. R. Acad. Sci. Paris 266, 596.

Bouck, G. B. (1965). J. Cell. Biol. 26, 523.

Bouck, G. B. (1969). J. Cell Biol. 40, 446.

Bouck, G. B. (1972). Advan. Cell Mol. Biol. 2, 237-271.

Bouck, G. B., and Brown, D. L. (1973). J. Cell Biol. 56, 340.

Bouteille, M. (1972). Exp. Cell Res. 74, 343.

Bouteille, M., Kalifat, S. R., and Delarue, J. (1967). J. Ultrastruct. Res. 19, 474.

Brachet, J., Hanocq, F., and Van Gansen, P. (1970). Develop. Biol. 21, 157.

Brachet, J., Huez, G., and Hubert, E. (1973). Proc. Nat. Acad. Sci. U.S. 70, 543.

Bracker, C. E., Heintz, C. E., and Grove, S. N. (1970). Sept. Congr. Int. Microsc. Electron. Grenoble 3, 103.

Brandt, P. W, and Pappas, G. D. (1959). J. Biophys. Biochem. Cytol. 6, 91.

Branton, D. (1966). Proc. Nat. Acad. Sci. U.S. 55, 1048.

Branton, D., and Deamer, D. W. (1972). "Membrane Structure," pp. 1-70. Springer: Wien.

Branton, D., and Moor, H. (1964). J. Ultrastruct. Res. 11, 401.

Brasch, K., Seligy, V. L., and Setterfield, G. (1971). Exp. Cell Res. 65, 61.

Breathnach, A. S., Stolinski, C., and Gross, M. (1972). J. Cell Sci. 11, 477.

Brenner, S. (1953). Exp. Cell Res. 5, 257.

Brökelmann, J. (1963). Z. Zellforsch. 59, 820.

Brown, R. M., and Franke, W. W. (1971). Planta 96, 354.

Brown, R. M., Johnson, C., and Bold, H. C. (1968). J. Phycol. 4, 100.

Brown, W. V., and Bertke, E. M. (1969). “Textbook of Cytology.” Mosby, St. Louis, Missouri.

Bucciarelli, E. (1966). J. Cell Biol. 30, 664.

Büttner, D. W., and Horstmann, E. (1967). Z. Zellforsch. 77, 589.

Burgess, J. (1970). Protoplasma 71, 77.

Burr, F. A., and West, J. A. (1971). J. Phycol. 7, 108.

Busch, H., and Smetana, K. (1970). "The Nucleolus." Academic Press, New York.

Buvat, R. (1963). Int. Rev. Cytol. 14, 41.

Calarco, P. G., and Brown, E. H. (1969). J. Exp. Zool. 171, 253.

Calarco, P. G., Donahue, R. P., and Szollosi, D. (1972). J. Cell Sci. 10, 369.

Callan, H. G. (1952). Symp. Soc. Exp. Biol. 6, 243.

Callan, H. G., and Tomlin, S. G. (1950). Proc. Roy. Soc. London B 137, 367.

Carlsson, S.-A., Moore, G. P. M., and Ringertz, N. R. (1973). Exp. Cell Res. 76, 234.

Carothers, Z. B. (1972a). Science 175, 652.

Carothers, Z. B. (1972b). J. Cell Biol. 52, 273.

Carr, I. (1967). Z. Zellforsch. 80, 140.

Carroll, G. (1967). J. Cell Biol. 33, 218.

Carroll, G. (1969). Arch. Mikrobiol. 66, 321.

Cassier, M. P., and Fain-Maurel, M.-A. (1968). C. R. Acad. Sci. Paris 266, 686.

Cave, M. D., and Allen, E. R. (1971). Z. Zellforsch. 120, 309.

Century, T. J., Fenichel, I. R., and Horowitz, S. B. (1970). J. Cell Sci. 7, 5.

Chang, J. P., and Gibley, C. W. (1968). Cancer Res. 28, 521.

Chardonnet, Y., and Dales, S. (1970). Virology 40, 478.

Chinn, P. (1938). J. Cell. Comp. Physiol. 12, 1. 
Clark, W. H. ( 1960$)$. J. Biophys. Biochem. Cytol. 7, 345.

Claude, A. (1964). In "Cellular Control Mechanisms and Cancer" (P. Emmelot and O. Mühlbock, eds.) pp. 41-48. Elsevier, Amsterdam.

Clérot, J.-C. (1968). J. Microsc. 7, 973.

Cleveland, L. R. (1938). Biol. Bull. 74, 1.

Cleveland, L. R. (1957a). J. Protozool. 4, 230.

Cleveland, L. R. (1957b). J. Protozool. 4, 241.

Coggeshall, R. E., and Fawcett, D. W. (1964). J. Neurophysiol. 27, 229.

Cole, M. B. (1969). J. Cell Biol. 43, 24 a.

Comes, P., and Franke, W. W. (1970). Z. Zellforsch. 107, 240.

Comings, D. E. (1968). Amer. J. Human Genet. 20, 440.

Comings, D. E., and Kakefuda, T. (1968). J. Mol. Biol. 33, 225.

Comings, D. E., and Okada, T. A. (1970a). Exp. Cell Res. 62, 293.

Comings, D. E., and Okada, T. A: (1970b). Chromosorna 30, 269.

Comings, D. E., and Okada, T. A. (1970c). Exp. Cell Res. 63, 62.

Comings, D. E., and Okada, T. A. (1970d). Exp. Cell Res. 63, 471.

Comings, D. E., and Okada, T. A. (1971). Exp. Cell Res. 67, 97.

Comings, D. E., and Okada, T. A. (1972a). Advan. Cell Mol. Biol. 2, 310-384.

Comings, D. E., and Okada, T. A. (1972b). J. Ultrastruct. Res. 39, 15.

Conway, C. M. (1971). J. Cell Biol. 51, 889.

Crawley, J. W. C. (1966). Planta 69, 365.

Cronshaw, J. (1967). Planta 72, 78.

Coulson, A. S., and Kennedy, L. (1972). Proc. Leucocyte Culture Conf. 6th pp. 91-104. Academic Press, New York.

Daniels, E. W., and Breyer, E. P. (1967). J. Protozool. 14, 167.

Daniels, E. W., Sustarsic, P. A., and Jacobs, G. B. (1968). Cancer Res. 28, 1207.

Daniels, E. W., McNiff, J. M., and Ekberg, D. R. (1969). Z. Zellforsch. 98, 357.

Darlington, R. W., and Moss, L. H. (1968). J. Virol. 2, 48.

Darlington, R. W., and Moss, L. H. (1969). Progr. Med. Virol. 11, 16.

David, H. (1959). Acta. Biol. Med. Germ. 3, 330.

David, H. (1964). Z. Mikrosk.-Anat. Forsch. 71, 526.

Davidson, E. H., Allfrey, V. G., and Mirsky, A. E. (1964). Proc. Nat. Acad. Sci. U.S. 52, 501.

Davies, H. G. (1967). Nature (London) 214, 208.

Davies, H. G. (1968). J. Cell Sci. 3, 129.

Davies, H. G., and Small, J. V. (1968). Nature (London) 217, 1122.

Dawson, J. M., Hossack, J., and Wyburn, G. M. (1955). Proc. Roy. Soc. B 144, 132.

De La Torre, C., Risueno, M. C., and Giménez-Martin, G. (1973). Protoplasma 76, 363.

Delektorskaya, L. N., and Perevoshchikova, K. A. (1969). Biokhimiya 34, 199.

Denis, H., and Mairy, M. (1972). Eur. J. Biochem. 25, 524.

DePetris, S., Karlsbad, G., and Pernis, B. (1963). J. Exp. Med. 117, 849.

DeRobertis, E. (1954). J. Histochem. Cytochem. 2, 341.

Deumling, B. (1972). Thesis, Univ. of Freiburg, pp. 1-139.

Deumling, B., and Franke, W. W. (1972). Hoppe-Seyler's Z. Physiol. Chem. 353, 287.

Deumling, B., and Franke, W. W. (1974). In preparation.

DeZoeten, G. A., and Gaard, G. (1969). J. Cell Biol. 40, 814.

Dhainaut, A. (1970a). J. Micros. 9, 99.

Dhainaut, A. (1970b). Z. Zellforsch. 104, 375. 
Diers, L. ( 1967 ). Planta 72, 119.

Döbel, P. (1970). Biol. Zentralbl. 89, 481.

Drawert, H., and Mix, M. (1961). Z. Naturforsch. 16b, 546.

Duckett, J. G. (1973). J. Cell Sci. 12, 95.

DuPraw, E. J. (1965). Proc. Nat. Acad. Sci. U.S. 53, 161.

DuPraw, E. J. (1968). "Cell and Molecular Biology." Academic Press, New York.

DuPraw, E. J. (1970). "DNA and Chromosomes." Holt, New York.

DuPraw, E. J., and Bahr, G. F. (1969). J. Cell Biol. 43, 32a.

Eckert, W. A. (1972). Thesis, Univ. of Freiburg, pp. 1-158.

Eckert, W. A., Franke, W. W., and Scheer, U. (1972). Z. Zellforsch. 127, 230.

Eckert, W. A., Franke, W. W., and Scheer, U. (1974). In preparation.

Eddy, E. M., and Ito, S. (1971). J. Cell Biol. 49, 90.

Engelhardt, P., and Pusa, K. (1972). Nature New Biol. 240, 163.

Erlandson, R. A., and deHarven, E. (1971). J. Cell Sci. 8, 353.

Esau, K. (1972). Ann. Bot. 36, 703.

Esau, K., and Gill, R. H. (1969). Can. J. Bot. 47, 581.

Esau, K., and Gill, R. H. (197I). J. Ultrastruct. Res. 34, 144.

Esponda, P., and Stockert, J. C. (1972). Chromosoma 36, 150.

Esser, K. (1968). Experientia 24, 61.

Everingham, J. W. (1968a). J. Cell Biol. 37, 540.

Everingham, J. W. (1968b). J. Cell Biol. 37, 551.

Evert, R. F., and Deshpande, B. P. (1970). J. Cell Biol. 44, 462.

Fabergé, A. C. (1973). Z. Zellforsch. 136, 183.

Fahimi, H. D. (1970). J. Cell Biol. 47, 247.

Fakan, S., and Bernhard, W. (1971). Exp. Cell Res. 67, 129.

Fakan, S., Turner, G. N., Pagano, J. S., and Hancock, R. (1972). Proc. Nat. Acad. Sci. U.S. 69, 2300.

Falk, H. (1967). Arch. Mikrobiol. 58, 212.

Falk, H. (1969), Ber. Deutsch. Bot. Ges. 82, 427.

Falk, H., and Kleinig, H. (1968). Arch. Mikrobiol. 61, 347.

Falk, H., Wunderlich, F., and Franke, W. W. (1968). J. Protozool. 15, 776.

Favard, P. (1961). Ann. Sci. Nat. Zool. (ser. 12) 3, 53.

Fawcett, D. W. (1958). Int. Rev. Cytol. 7, 195.

Fawcett, D. W. (1964). In "Modern Developments in Electron Microscopy" (B. M.

Siegel, ed.), pp. 257-333. Academic Press, New York.

Fawcett, D. W. (1966a). Amer. J. Anat. 119, 129.

Fawcett, D. W. (1966b). "An Atlas of Fine Structure. The Cell." Saunders, Philadelphia, Pennsylvania.

Fawcett, D. W. (1970). Biol. Reprod. Suppl. 22, 90.

Fawcett, D. W. (1972). In "Proc. Int. Symp. The Genetics of the Spermatozoon," Edinburgh, 1971 (R. A. Beatty and S. Gluecksohn-Waelsch, eds.), pp. 37-68. Edinburgh, New York.

Fawcett, D. W., and McNutt, N. S. (1969). J. Cell Biol. 42, 1.

Fawcett, D. W., and Phillips, D. M. (1970). In "Comparative Spermatology" (B. Baccetti, ed.), pp. 13-28. Academic Press, New York.

Fawcett, D. W., Eddy, E. M., and Phillips, D. M. (1970). Biol. Reproduct. 2, 129.

Fawcett, D. W., Anderson, W. A., and Phillips, D. M. (1971). Develop. Biol. 26, 220.

Feldherr, C. M. (1964). J. Cell Biol. 20, 188.

Feldherr, C. M. (1965). J. Cell Biol. 25, 43.

Feldherr, C. M. (1966). J. Cell Biol. 31, 199. 
Feldherr, C. M. (1968a). J. Cell Biol. 39, 49.

Feldherr, C. M. (1968b). Nature (London) 218, 184.

Feldherr, C. M. (1969). J. Cell Biol. 42, 841.

Feldherr, C. M. (1972). Advan. Cell Mol. Biol. 2, 273-307.

Feldherr, C. M., and Harding, C. V. (1964). Protoplasmatologia (Vienna) 5, 35.

Feldman, M. (1968). Proc. Int. Wheat Genet. Symp., 3rd Canberra 1968 p. 169. Aust. Acad. Sci.

Feldman, M., Mello-Sampayo, T., and Sears, E. R. (1966). Proc. Nat. Acad. Sci. U.S. 56, 1192.

Feldman, M., Mello-Sampayo, T., and Avivi, L. (1972). Chromosoma 37, 209.

Fiil, A., and Moens, P. B. (1973). Chromosoma 43, 37.

Fisher, H. W., and Cooper, T. W. (1967). Exp. Cell Res. 48, 620.

Fleischer, S., Fleischer, B., Azzi, A., and Chance, B. (1971). Biochim. Biophys. Acta 225, 194.

Flickinger, C. J. (1970). Exp. Cell Res. 60, 225.

Folliot, R (1968). Z. Zellforsch. 92, 115.

Folliot, R., and Picheral, B. (1971). J. Microsc. 10, 219.

Forte, G. M., Limlomwongse, L., and Forte, J. G. (1969). J. Cell Sci. 4, 709.

Fowke, L. C., and Pickett-Heaps, J. D. (1969). J. Phycol. 5, 240.

Franke, W. W. (1966a). Diplomarbeit. Faculty of Sciences, Univ. of Heidelberg, pp. $1-46$.

Franke, W. W. (1966b). J. Cell Biol. 31, 619.

Franke, W. W. (1967a). Thesis, Univ. of Heidelberg, pp. I-82.

Franke, W. W. (1967b). Z. Zellforsch. 80, 585.

Franke, W. W. (1970a). Z. Zellforsch. 105, 405.

Franke, W. W. (1970b). Naturwissenschaften 57, 44.

Franke, W. W. (1970c). Naturwissenschaften 57, 503.

Franke, W. W. (1970d). Z. Zellforsch. 111, 143.

Franke, W. W. (1971a). Protoplasma 73, 263.

Franke, W. W. (1971b). Exp. Cell Res. 66, 486.

Franke, W. W. (1971c). Cytobiologie 4, 150.

Franke, W. W. (1971d). Z. Naturforsch. 26b, 626.

Franke, W. W. (1974). Int. Rev. Cytol. (in press).

Franke, W. W., and Falk, H. (1970). Histochemie 24, 266.

Franke, W. W., and Kartenbeck, J. (1969). Experientia 25, 396.

Franke, W. W., and Krien, S. (1972). Naturwissenschaften 59, 37.

Franke, W. W., and Reau, P. (1973). Arch. Mikrobiol. 90, 121.

Franke, W. W., and Scheer, U. (1970a). J. Ultrastruct. Res. 30, 288.

Franke, W. W., and Scheer, U. (1970b). J. Ultrastruct. Res. 30, 317.

Franke, W. W., and Scheer, U. (1971). Cytobiologie 4, 317.

Franke, W. W., and Scheer, U. (1972). J. Ultrastruct. Res. 40, 132.

Franke, W. W., and Schinko, W. (1969). J. Cell Biol. 42, 326.

Franke, W. W., Deumling, B., Ermen, B., Jarasch, E. D., Kleinig, H. (1970a). J. Cell Biol. 46, 379.

Franke, W. W., Morré, D. J., Deumling, B., Cheetham, R. D., Kartenbeck, J., Jarasch, E. D., and Zentgraf, H. (1971a). Z. Naturforsch. 26b, 1031.

Franke, W. W., Eckert, W. A., and Krien, S. (1971b). Z. Zellforsch. 119, 577.

Franke, W. W., Kartenbeck, J., Zentgraf, H., Scheer, U., and Falk, H. (1971c). J. Cell Biol. 51, 881.

Franke, W. W., Kartenbeck, J., and Deumling, B. (1971d). Experientia 27, 372.

Franke, W. W., Scheer, U., and Fritsch, H. (1972a). J. Cell Biol. 53, 823. 
Franke, W. W., Kartenbeck, J., Krien, S., Van der Woude, W. J., Scheer, U., and Morré, D. J. (1972b). Z. Zellforsch. 132, 365.

Franke, W. W., Zentgraf, H., Scheer, U., and Kartenbeck, J. (1973a). Cytobiologie, 7,89 .

Franke, W. W., Deumling, B., Zentgraf, H., Falk, H., and Rae, P. M. M. (1973b). Exp. Cell Res. 81, 365.

Franke, W. W., Berger, S., Spring, H., Herth, W., Falk, H., Scheer, U., and Schweiger, H.-G. (1974). In preparation.

Franklin, L. E. (1968). Anat. Rec. 161, 149.

Frasca, J. M., Auerbach, O., Parks, V. R., and Stöckenius, W. (1967). Exp. Mol. Pathol. 6, 261.

Friederici, H. H. R. (1968). J. Ultrastruct. Res. 23, 444.

Freidman, D. L., and Mueller, G. C. (1969). Biochim. Biophys. Acta 174, 253.

Friedman, M. H. (1971). J. Cell Biol. 49, 916.

Frey-Wyssling, A., and Mühlethaler, K. (1965). "Ultrastructural Plant Cytology." Elsevier, Amsterdam.

Fuge, H. (1971). Z. Zellforsch. 120, 579.

Fullilove, S. L., and Jacobson, A. G. (1971). Develop. Biol. 26, 560.

Fulton, C. (1971). In "Origin and Continuity of Cell Organelles" (J. Reinert and H. Ursprung, eds.), Vol. 2, pp. 170-221. Springer-Verlag, Heidelberg.

Fussell, C. P. (1972). J. Cell Biol. 55, 79a.

Gagné, R., and Labergé, C. (1972). Exp. Cell Res. 73, 239.

Gall, J. G. (1954). Exp. Cell Res. 7, 197.

Gall, J. G. (1956). J. Biophys. Biochem. Cytol. Suppl. $2,393$.

Gall, J. G. (1959). J. Biophys. Biochem. Cytol. 6, 115.

Gall, J. G. (1964). Protoplasmatologia (Vienna) 5, 4.

Gall, J. G. (1965). J. Cell Biol. 27, 121a.

Gall, J. G. ( 1967 ). J. Cell Biol. 32, 391.

Gall, J. G., Cohen, E. H., and Polan, M. L. (1971). Chromosoma 33, 319.

Gay, H. (1955). Proc. Nat. Acad. Sci. U.S. 41, 370.

Gay, H. (1956). J. Biophys. Biochem. Cytol. Suppl. $2,407$.

Gerin, Y. (1971). J. Embryol. Exp. Morphol. 25, 423.

Gianordoli, M. (1969). Rev. Cytol. Biol. Vég. 32, 183.

Gibbs, S. P. (1962). J. Cell Biol. 14, 433.

Gibbs, S. P. (1970). Ann. N.Y. Acad. Sci. 175, 454.

Girbardt, M. (1970). Z. Allg. Mikrobiol. 10, 451.

Glaumann, H., von der Decken, A., and Dallner, G. (1968). Life Sci. 7, 905.

Goldfischer, S., Essner, E., and Novikoff, A. B. (1964). J. Histochem. Cytochem. 12, 72 .

Goldstein, L. (1964). Protoplasmatologia (Vienna) 5, 51.

Goldstein, L. (1970a). Exp. Cell Res. 61, 218.

Goldstein, L. (1970b). Advan. Cell Biol. 1, 187.

Goldstein, L., and Prescott, D. M. (1967a). J. Cell Biol. 33, 637.

Goldstein, L., and Prescott, D. M. (1967b). In "The Control of Nuclear Activity" (L. Goldstein, ed.), p. 273. Prentice Hall, Englewood Cliffs, New Jersey.

Goldstein, L., Wise, G. E., and Beeson, M. (1973). Exp. Cell Res. 76, 281.

Gondos, B., Bhiraleus, P., and Conner, L. A. (1972). J. Cell Sci. 10, 61.

Gorovsky, M. A. (1969). J. Cell Biol. 43, 46a.

Gouranton, J. (1969). Ann. Biol. 8, 385.

Gray, E. G., and Guillery, R. W. (1963). Z. Zellforsch. 59, 738.

Grassé, P. P. (1952). “Traité de Zoologie," 1, fasc. 1. Masson, Paris. 
Green, B. R., and Burton, H. (1970). In "Biology of Acetabularia" ( J. Brachet and S. Bonotto, eds.), pp. 35-59. Academic Press, New York.

Green, S., and Dobrjansky, A. (1972). Biochemistry 11, 4108.

Greider, M. H., Kostir, W. J., and Frajola, W. J. (1956). J. Biophys. Biochem. Cytol. Suppl. 2, 445.

Grimstone, A. V. (1959). J. Biophys. Biochem. Cytol. 6, 369.

Grimstone, A. V., and Cleveland, L. R. (1965). J. Cell Biol. 24, 387.

Grove, S. N., Bracker, C. E., and Morré, D. J. (1968). Science 161, 171.

Grove, S. N., Bracker, C. E., and Morré, D. J. (1970). Amer. J. Bot. 57, 245.

Gulyas, B. J. (1971). Z. Zellforsch. 120, 151.

Gulyas, B. J. (1972a). J. Ultrastruct. Res. 35, 112.

Gulyas, B. J. (1972b). Z. Zellforsch. 133, 187.

Gurdon, J. B. (1969). Develop. Biol. Suppl. 3, 59.

Gurdon, J. B. (1970). Proc. Roy. Soc. London B 176, 303.

Gurr, M. I., Finean, J. B., and Hawthorne, J. N. (1963). Biochim. Biophys. Acta $70,406$.

Guttes, S., Guttes, E., and Ellis, R. A. (1968). J. Ultrastruct. Res. 22, 508.

Hadek, R., and Swift, H. (1962). J. Cell Biol. 13, 445.

Hallinan, T., Duffy, T., Waddington, S., and Munro, H. N. (1966). Quart. J. Exp. Physiol. 51, 142.

Hanaoka, H., and Friedman, B. (1970). J. Ultrastruct. Res. 32, 323.

Hanaoka, F., and Yamada, M. (1971). Biochem. Biophys. Res. Commun. 42, 647.

Hancock, R. (1969). J. Mol. Biol. 40, 457.

Hancock, R., and Ryser, H. J.-P. (1967). Nature (London) 213, 701.

Hanzely, L., and Olah, L. V. (1973). Trans. Amer. Microsc. Soc. 92, 35.

Harris, J. R., and Agutter, P. (1970). J. Ultrastruct. Res. 33, 219.

Harris, J. R., and Brown, J. N. (1971). J. Ultrastruct. Res. 36, 8.

Harris, P. (1961). J. Biophys. Biochem. Cytol. 11, 419.

Harris, P. (1967). Exp. Cell Res, 48, 569.

Harris, P. (1969). In "The Cell Cycle" (G. M. Padilla, G. L. Whitson, and I. L. Cameron, eds.), pp. 315-340. Academic Press, New York.

Harris, P., and James, T. W. (1952). Experientia 8, 384.

Harris, P., and Mazia, D. (1962). In "The Interpretation of Ultrastructure" (R. J. C. Harris, ed.), Symp. Int. Soc. Cell Biol, Vol. 1. pp. 279-305. Academic Press, New York.

Harrison, G. A. (1966). J. Ultrastruct. Res. 14, 158.

Hatfield, J. M. R. (1972). Exp. Cell Res. 72, 591.

Haynes, M. E., and Davies, H. G. (1973). J. Cell Sci. 13, 139.

Heath, I. B., and Greenwood, A. D. (1968). J. Gen. Microbiol. 53, 287.

Heath, I. B., and Greenwood, A. D. (1970). J. Gen. Microbiol. 62, 139.

Heath, I. B., and Greenwood, A. D. (1971). Z. Zellforsch. 112, 371.

Heath, I. B., Greenwood, A. D., and Griffiths, H. B. (1970). J. Cell Sci. 7, 445.

Hecht, N. B., and Stern, H. (1969). J. Cell Biol. 43, 51 a.

Heitz, E. (1932). Planta 18, 571.

Hepler, P. K., McIntosh, J. R., and Cleland, S. (1970). J. Cell Biol. 45, 438.

Herrlinger, H. (1970). Ergeb. Anat. Entwickl. Ges. 42, 1. (Rev. Anat. Embryol. Cell Biol.)

Hertig, A. T., and Adams, E. C. (1967). J. Cell Biol. 34, 647.

Hertwig, O. ( 1893). "Die Zelle und die Gewebe." Fischer Verlag, Jena.

Herzog, V., and Miller, F. (1970). Z. Zellforsch. 107, 403. 
Herzog, V., and Miller, F. (1972). J. Cell Biol. 53, 662.

Hibberd, D. J. (1970). Brit. Phycol. J. 5, 119.

Hinsch, G. W. (1970). J. Cell Biol. 47, 531.

Hoage, T. R., and Kessel, R. G. (1968). J. Ultrastruct. Res. 24, 6.

Hönigsmann, H., and Wolff, K. (1973). Exp. Cell Res. 80, 191.

Hollande, A., and Valentin, J. (1968a). C. R. Acad. Sci. Paris 266, 367.

Hollande, A., and Valentin, J. (1968b). C. R. Acad. Sci. Paris 267, 1383.

Hollande, A., and Carruette-Valentin, J. (1971). Protistologica 7, 5.

Hollande, A., Cachon, J., and Cachon, M. (1969). C. R. Acad. Sci. Paris Ser. D 269, 179.

Holmes, K. V., and Choppin, P. W. (1968). J. Cell Biol. 39, 526.

Horowitz, S. B. (1972). J. Cell Biol. 54, 609.

Horowitz, S. B., and Fenichel, I. R. (1968). J. Gen. Physiol. 51, 703.

Horowitz, S. B., and Fenichel, I. R. (1970). J. Cell Biol. 47, 120.

Horstmann, E. (1961). Z. Zellforsch. 54, 68.

Horstmann, E., and Breucker, H. (1969). Z. Zellforsch. 99, 153.

Horstmann, E., and Knoop, A. (1957). Z. Zellforsch. 46, 100.

Howard, K. L., and Moore, R. T. (1970). Bot. Gas. 131, 311.

Hsu, T. C., Cooper, J. E. K., Mace, M. L., and Brinkley, B. R. (1971). Chromosoma $34,73$.

Hsu, W. S. (1963). Z. Zellforsch. 58, 660.

Hsu, W. S. (1967). Z. Zellforsch. 82, 376.

Huhn, D. (1967). Nature (London) 216, 1240.

Ichida, A. A., annd Fuller, M. S. (1968). Mycologia 60, 141.

Ichikawa, Y., and Mason, H. S. (1973). Proc. 2nd Int. Symp. Oxydases Related Redox Syst., Univ. Park Press, Baltimore, Maryland (in press).

Inaba, F, and Sotokawa, Y. (1968). Jap. J. Genet. 43, 335.

Ito, S., and Loewenstein, W. R. (1965). Science 150, 909.

Ito, J., Lee, Y. C., and Scherbaum, O. H. (1968). Exp. Cell Res. 53, 85.

Izquierdo, L., and Vial, J. D. (1962). Z. Zellforsch. 56, 157.

Jackson, V., Earnhardt, J., and Chalkley, R. (1968). Biochem. Biophys. Res. Commun. 33, 253.

Jacob, F., Brenner, S., and Cuzin, F. (1963). Cold Spring Harbor Symp. Quant. Biol. 28, 329.

Jacob, J., and Danieli, G. A. (1972). Cell Different. 1, 119.

Jacob, J., and Jurand, A. (1963). Chromosoma 14, 451.

Jansz, H. S., van der Mei, D., and Zandvliet, G. M. (1971). Int. Rev. Cytol. $31,115$.

Jarasch, E.-D. ( 1969). Diplomarbeit, Univ. of Freiburg, pp. 1-41.

Jarasch, E. D., and Franke, W. W. (1974). J. Biol. Chem. (in press).

Jarasch, E. D., Reilly, C. E., Comes, P., Kartenbeck, J., and Franke, W. W. (1973). Hoppe-Seyler's Z. Physiol. Chem. 354, 974.

Jenkins, R. A. (1967). J. Cell Biol. 34, 463.

Jensen, W. A. (1964). J. Cell Biol. 23, 669.

Johnson, U. G., and Porter, K. R. (1968). J. Cell Biol. 38, 403.

Jollie, W. P. (1969). Anat. Rec. 165, 1.

Jones, K. W., Prosser, J., Corneo, G., and Ginelli, E. (1973). Chromosoma 42, 445.

Joyon, L. (1963). Ann. Faculté Sci. Univ. Clermont 22, 1.

Jurand, A., and Selman, G. G. (1970). J. Gen. Microbiol. 60, 357.

Kalifat, S. R., Bouteille, M., and Delarue, J. (1967). J. Microsc. 6, 10.

Kanno, Y., and Loewenstein, W. R. (1963). Exp. Cell Res. 31, 149. 
Kanno, Y., Ashman, R. F., and Loewenstein, W. R. (1965). Exp. Cell Res. 39, 184.

Karasaki, S. (1971). J. Ultrastruct. Res. 35, 181.

Kartenbeck, J., Zentgraf, H., Scheer, U., and Franke, W. W. (1971). Ergeb. Anat. Entwickl. Gesch. 45, 1. (Rev. Anat. Embryol. Cell Biol.)

Kartenbeck, J., Jarasch, E. D., and Franke, W. W. (1973). Exp. Cell Res. 81, 175. Kashnig, D. M., and Kasper, C. B. (1969). J. Biol. Chem. 244, 3786.

Kasper, C. B. (1971). J. Biol. Chem. 246, 577.

Kawasaki, T., and Yamashina, I. (1972). J. Biochem. 72, 1517.

Kay, R. R., Haines, M. E., and Johnston, I. R. (1971). FEBS Lett. 16, 233.

Kay, R. R., Fraser, D., and Johnston, I. R. (1972). Eur. J. Biochem. 30, 145.

Keenan, T. W., and Morré, D. J. (1970). Biochemistry 9, 19.

Keenan, T. W., Berezney, R., Funk, L. K., and Crane, F. L. (1970). Biochim. Biophys. Acta 203, 547.

Keenan, T. W., Berezney, R., and Crane, F. L. (1972). Lipids 7, 212.

Kelley, R. O. (1972). J. Ultrastruct. Res. 40, 325.

Kessel, R. G. (1963). J. Cell Biol. 19, 391.

Kessel, R. G. (1964). Z. Zellforsch. 63, 37.

Kessel, R. G. (1966). J. Ultrastruct. Res. 16, 293.

Kessel, R. G. (1968a). J. Ultrastruct. Res. Suppl. 10, 1.

Kessel, R. G. (1968b). J. Ultrastruct. Res. 22, 63.

Kessel, R. G. (1968c). J. Cell Biol. 36, 658.

Kessel, R. G. (1969a). Z. Zellforsch. 94, 441.

Kessel, R. G. (1969b). Z. Zellforsch. 94, 454.

Kessel, R. G. (1970). In "Comparative Spermatology" (B. Baccetti, ed.), pp. 531-552. Academic Press, New York.

Kessel, R. G. (1971). J. Ultrastruct. Res. 34, 260.

Kessel, R. G., and Beams, H. W. (1963). Exp. Cell Res. 32, 612.

Kessel, R. G., and Beams, H. W. (1968). J. Cell Biol. 39, 735.

Kessel, R. G., and Beams, H. W. (1969). J. Cell Biol. 42, 185.

Kezer, J., MacGregor, H. C., and Schabtach, E. (1971). J. Cell Sci. 8, 1.

Kiermayer, O. (1968). Planta 83, 223.

Kiermayer, O. (1971). Planta 96, 74.

Kilarski, W., and Jasinski, A. (1970). J. Cell Biol. 45, 205.

King, P. E., and Fordy, M. R. (1970). Z. Zellforsch. 109, 158.

King, R. C., and Devine, R. L. (1958). Growth 22, 299.

Kitajima, E. W., Lauritis, J. A., and Swift, H. (1969). J. Ultrastruct. Res. 29, 141.

Klein, R. L., and Afzelius, B. A. (1966). Nature (London) 212, 609.

Kleinig, H. (1970). J. Cell Biol. 46, 396.

Kleinig, H., Zentgraf, H., Comes, P., and Stadler, J. (1971). J. Biol. Chem. 246, 2996.

Kohen, E., Siebert, G., and Kohen, C. (1971). Hoppe-Seyler's Z. Physiol. Chem. $352,927$.

Konings, A. W. T., and Loomeijer, F. J. (1970). Biochim. Biophys. Acta 202, 216.

Koshiba, K., Smetana, K., and Busch, H. (1970). Exp. Cell Res. 60, 199.

deKretser, D. M. (1969). Z. Zellforsch. 98, 477.

Kubai, D. F., and Ris, H. (1969). J. Cell Biol. 40, 508.

Kubinski, H., Gibbs, S. P., and Kasper, C. B. (1972). Biochim. Biophys. Acta 281, 244. 
Kuroiwa, T., and Tanaka, N. (1971). J. Cell Biol. 49, 939.

Kuzmina, S. N., Zbarsky, I. B., Monakhov, N. K., Gaitzkhoki, V. S., and Neifakh, S. A. (1969). FEBS Lett. 5, 34.

LaCour, L. F., and Wells, B. (1972). Z. Zellforsch. 123, 178.

LaFountain, J. R. (1972). Exp. Cell Res. 71, 325.

LaFountain, J. R., and LaFountain, K. L. (1973). Exp. Cell Res. 78, 472.

Lambert, A.-M. (1970). C. R. Acad. Sci. Paris 270, 481.

Lampert, F. (1971). Humangenetik 13, 285.

Lane, N. J. (1967). J. Cell Biol. 35, 421.

Lang, N. J. ( 1963). Amer. J. Bot. 50, 280.

Langreth, S. G. (1969). J. Cell Biol. 43, 575.

Lanzavecchia, G., and Donin, C. L. L. (1972). J. Submicrosc. Cytol. 4, 247.

Lasek, R. J., Lee, C. K., and Przybylski, R. J. ( 1972). J. Cell Biol. 55, 237.

Leadbeater, B., and Dodge, J. D. (1967). Arch Mikrobiol. 57, 239.

LeBeux, Y. J. (1972). Z. Zellforsch. 133, 327.

Ledbetter, M. C. (1967). In "Formation and Fate of Cell Organelles" (K. B. Warren, ed.), pp. 55-70. Academic Press, New York.

Leduc, E. H., Avrameas, S., and Bouteille, M. (1968). J. Exp. Med. 127, 109.

Leduc, E. H., Scott, G. B., and Avrameas, S. (1969). J. Histochem. Cytochem. $17,211$.

Leedale, G. F. (1969). Osterr. Bot. Z. 116, 279.

Leedale, G. F., Leadbeater, B., and Massalski, A. (1970). J. Cell Sci. 6, 701.

Legname, C., and Goldstein, L. (1972). Exp. Cell Res. 75, 111.

Legrand, C., and Hernandez-Verdun, D. (1971). Cytobiologie 4, 198.

Lemarchal, P., and Bornens, M. (1969). Bull. Soc. Chim. Biol. 51, 1021.

Leskes, A., and Siekevitz, P. (1969). J. Cell Biol. 43, 80a.

Lessie, P. E., and Lovett, J. S. (1968). Amer. J. Bot. 55, 220.

Lettré, H., and Lettré, R. (1959). Nucleus 2, 23.

Lima-de-Faria, A. (1959). J. Biophys. Biochem. Cytol. 6, 457.

Lima-de-Faria, A. (1969). In "Handbook of Molecular Cytology" A. Lima-de-Faria, ed. ), pp. 277-325. North-Holland Publ., Amsterdam.

Lima-de-Faria, A. (1971). Lecture given at the 2nd Eur. Nucleolar Workshop, Lake Balaton (Hungary).

Loening, U. E. (1968). J. Mol. Biol. 38, 355.

Loewenstein, W. R. (1964). Protoplasmatologia (Vienna) 5, 26.

Loewenstein, W. R., and Kanno, Y. (1963a). J. Cell Biol. 16, 421.

Loewenstein, W. R., and Kanno, Y. (1963b). J. Gen. Physiol. 46, 1123.

Longo, F. J., and Anderson, E. (1968). J. Cell Biol. 39, 339.

Longo, F. J., and Anderson, E. (1969). J. Ultrastruct. Res. 29, 86.

Longo, F. J., and Anderson, E. (1970a). J. Ultrastruct. Res. 32, 94.

Longo, F. J., and Anderson, E. (1970b). J. Ultrastruct. Res. 33, 495.

Longo, F. J., and Anderson, E. (1970c). J. Ultrastruct. Res. 33, 515.

Lott, J. N. A., Larsen, P. L., and Whittington, C. M. (1972). Can. J. Bot. 50, 1785.

Løvlie, A., and Braten, T. (1970). J. Cell Sci. 6, 109.

Lukanidin, E. M., Zalmanzon, E. S., Komaromi, L., Samarina, O. P., and Georgiev, G. P. (1972). Nature New Biol. 238, 193.

Luykx, P. (1970). "Cellular Mechanisms of Chromosome Distribution." Academic Press, New York.

MacGregor, H. C., and Kezer, J. (1971). Chromosoma 33, 167.

MacGregor, H. C., and Walker, M. H. (1973). Chromosoma 40, 243. 
MacKinnon, E. A., and Abraham, J. P. (1972). Z. Zellforsch. 124, 1.

Mahowald, A. P. (1971a). J. Exp. Zool. 176, 345.

Mahowald, A. P. (I971b). In "Origin and Continuity of Cell Organelles" (J. Reinert and H. Ursprung, eds.) pp. 158-169. Springer-Verlag, Berlin and New York.

Mancuso, V. (1964). Acta Embryol. Morphol. Exp. 7, 269.

Mancuso, V. (1972). Acta Embryol. Exp. 93.

Manton, I. (1964). J. Roy. Micros. Soc. 83, 471.

Manton, I., Kowallik, K., and von Stosch, H. A. (1969a). J. Microsc. (London) 89, 295.

Manton, I., Kowallik, K., and von Stosch, H. A. (1969b). J. Cell Sci. 5, 271.

Marchant, H. J., and Pickett-Heaps, J. D. (1970). Aust. J. Biol. Sci. 23, 1173.

Markham, R. S., Frey, S., and Hills, G. J. (1963). Virology 20, 88.

Marquet, E., and Sobel, H. J. (1969). J. Cell Biol. 41, 774.

Massalski, A., and Leedale, G. F. (1969). Brit. Phycol. J. 4, 159.

Matsui, S.-I., Yoshida, H., Weinfeld, H., and Sandberg, A. A. (1972). J. Cell Biol. 54, 120.

Matsuura, T., and Ueda, K. (1972). Arch. Biochem. Biophys. 150, 440.

Maui, G. G. (1970a). J. Ultrastruct. Res. 30, 368.

Maul, G. G. (1970b). J. Ultrastruct. Res. 31, 375.

Maul, G. G. (1971a). J. Cell Biol. 51, 558.

Maul, G. G. (197Ib). J. Ultrastruct. Res. 36, 768.

Maul, G. G., Price, J. W., and Lieberman, M. W. (1971). J. Cell Biol. 51, 405.

Maul, G. G., Maul, H. M., Scogna, J. E., Lieberman, M. W., Stein, G. S., Hsu, B. Y., and Borun, T. W. (1972). J. Cell Biol. 55, 433.

McAlear, J. H., and Edwards, G. A. (1959). Exp. Cell Res. 16, 689.

McCully, E. K., and Robinow, C. F. (1971). J. Cell Sci. 9, 475.

McCully, E. K., and Robinow, C. F. (1972a). J. Cell Sci. 10, 857

McCully, E. K., and Robinow, C. F. (1972b). J. Cell Biol. 11, 1.

McDonald, K. (1972). J. Phycol. 8, 156.

McIntosh, J. R., and Porter, K. R. (1967). J. Cell Biol. 35, 153.

McIntosh, J. R., Hepler, P. K.; and VanWie, D. G. (1969). Nature (London) 224, 659 .

Mazanec, K. (1967). J. Microsc. 6, 1027.

Meek, G. A., and Moses, M. J. (1961). J. Biophys. Biochem. Cytol. 10, 121.

Mentré, P. (1966). Int. Congr. Electron Microsc. 6th Kyoto, 1966 2, 347. Maruzen Co., Tokyo.

Mentré, P. (1969). J. Micros. 8, 51.

Mepham, R. H., and Lane, G. R. (1969). Nature (London) 221, 288.

Mercer, E. H. (1959). Proc. Roy. Soc. B 150, 216.

Merkow, L. P., Slifkin, M., Pardo, M., and Rapoza, N. P. (1970). J. Ultrastruct. Res. 30, 344.

Merriam, R. W. (1959). J. Biophys. Biochem. Cytol. 5, 117.

Merriam, R. W. (1961). J. Biophys. Biochem. Cytol. 11, 559.

Merriam, R. W. (1962). J. Cell Biol. 12, 79.

Merriam, R. W. (1969). J. Cell Sci. 5, 333.

Meyer, G. F. (1963). Chromosoma 14, 207.

Mignot, J.-P. (1965). J. Microsc. 4, 239.

Mignot, J.-P. ( 1967). Protistologica 3, 5.

Mignot, J.-P., Brugerolle, G., and Metenier, G. (1972). J. Micros. 14, 327.

Miller, O. L., Jr. (1966). Nat. Cancer Inst. Monogr. 23, 53. 
Miller, O. L., Jr., and Beatty, B. R. (1969). Science 164, 955.

Millonig, G., Bosco, M., and Giambertone, L. (1968). J. Exp. Zool. 169, 293.

Mizuno, N. S., Stoops, C. E., and Sinha, A. A. (1971a). Nature New Biol. (London) $229,22$.

Mizuno, N. S., Stoops, C. E., and Pfeiffer, R. L. (1971b). J. Mol. Biol. 59, 517.

Moens, P. B. (1969). Chromosoma 28, 1.

Moens, P. B., and Perkins, F. O. (1969). Science 166, 1289.

Moens, P. B., and Rapport, E. (1971). J. Cell Biol. 50, 344.

Mollo, F., Canese, M. G., and Stramignoni, A. (1969). Nature (London) 221, 869.

Monneron, A., and Bernhard, W. (1969). J. Ultrastruct. Res. 27, 266.

Monneron, A., Blobel, G., and Palade, G. E. (1972). J. Cell Biol. 55, 104.

Monroe, J. H., Schidlovsky, G., and Chandra, S. (1967). J. Ultrastruct Res. 21, 134.

Moor, H. (1966). J. Cell Biol. 29, 153.

Moor, H. (1967). Protoplasma 64, 89.

Moor, H., and Mühlethaler, K. (1963). J. Cell Biol. 17, 609.

Moore, R. T., and McAlear, J. H. (1963). J. Cell Biol. 16, 131.

Moores, R T., and Wilson, J. D. (1972). J. Biol. Chem. 247, 958.

Morgan, C., Rosenkranz, H. S., and Mednis, B. (1969). J. Virol. 4, 777.

Mori, M., Dempo, K., Abe, M., and Onoe, T. (1970). J. Electron Micros. 19, 163.

Morré, D. J., Mollenhauer, H. H., and Bracker, C. E. (1971a). In "Origin and Continuity of Cell Organelles" (J. Reinert and H. Ursprung, eds.), pp. 82-126. Springer Verlag, Berlin.

Morré, D. J., Franke, W. W., Deumling, B., Nyquist, S. E., and Ovtracht, L. (1971b). In "Biomembranes" (L. A. Manson, ed.) Vol. 2, pp. 95-104. Plenum Press, New York.

Moses, M. J. (1960a). Int. Conf. Electron Micros., 4th 1958 p. 199. Springer Verlag, Berlin.

Moses, M. J. (1960b). Int. Conf. Electron Micros., 4th 1958 p. 230. Springer Verlag, Berlin.

Moses, M. J. (1968). Ann. Rev. Gen. 2, 363.

Moses, M. J., and Coleman, J. R. (1964). In "The Role of Chromosomes in Development," (M. Locke, ed.) pp. 11-49. Academic Press, New York.

Moses, M. J., and Wilson, M. H. (1970). Chromosoma 30, 373.

Mottier, D. M. (1899). Ann. Bot. 13, 325.

Munk, K., and Waldeck, C. (1969). Naturwissenschaften 56, 567.

Murray, R. G., Murray, A. S., and Pizzo, A. (1965). J. Cell Biol. 26, 601.

Naora, H., Izawa, M., Allfrey, V. G., and Mirsky, A. E. (1962). Proc. Nat. Acad. Sci. U.S. $48,853$.

Nass, M. M. K., and Nass, S., (1963). J. Cell Biol. 19, 593.

Nass, M. M. K., Nass, S., and Afzelius, B. A. (1965). Exp. Cell Res. 37, 516.

Neushul, M. (1970). Amer. J. Bot. 57, 1231.

Neushul, M., and Dahl, A. L. (1972). Amer. J. Bot. 59, 401.

Neushul, M., and Walker, D. C. (1971). J. Phycol. (Suppl.) 7, 3.

Nii, S., Morgan, C., and Rose, H. M. (1968). J. Virol. 2, 517.

Norberg, B. (1969). Scan. J. Haemat. 6, 312.

Norberg, H. S. (1970). Z. Zellforsch. 110, 61.

Nørrevang, A. (1965). Vidensk. Medd. fra Dansk. natur. Foren. 128, 1.

Northcote, D. H., and Lewis, D. R. (1968). J. Cell Sci. 3, 199. 
Novikoff, A. B. (1957). Cancer Res. 17, 1010.

O'Brien, R. L., Sanyal, A. B., and Stanton, R. H. (1972). Exp. Cell Res. 70, 106. Ockey, C. H. (1972). Exp. Cell Res. 70, 203.

Odintsova, M. S., and Turischeva, M. S. (1972). In "Nucleic Acids and Proteins in Higher Plants” (G. L. Farkas, ed.), pp. 221-227. Akadémiai Kiadó, Budapest. Okada, E., and Waddington, C. H. (1959). J. Embryol. Exp. Morphol. 7, 583.

Ollerich, D. A., and Carlson, E. C. (1970). J. Ultrastruct. Res. 30, 411.

Olson, L. W., and Kochert, G. (1970). Arch. Mikrobiol. 74, 31.

Orci, L., Perrelet, A., and Like, A. A. (1972). J. Cell Biol. 55, 245.

Ormerod, M. G., and Lehmann, A. R. (1971). Biochim. Biophys. Acta 228, 331.

Ornstein, L. (1956). J. Biophys. Biochem. Cytol. Suppl. 2, 351.

Ott, D. W., and Brown, R. M. (1973). Brit. Phycol. J. 7, 361.

Outka, D. E., and Kluss, B. C. (1967). J. Cell Biol. 35, 323.

Paine, P. L., and Feldherr, C. M. (1972). Exp. Cell Res. 74, 81.

Palade, G. E. (1955). J. Biophys. Biochem. Cytol. 1, 567.

Palade, G. E., and Bruns, R. R. (1968). J. Cell Biol. 37, 633.

Pankratz, H. S. (1967). J. Cell Biol. 35, 155a.

Paolillo, D. J., Kreitner, G. L., and Reighard, J. A. (1968). Planta 78, 226.

Pappas, G. D. (1956). J. Biophys. Biochem. Cytol. Suppl. 2, 431.

Patrizi, G., and Poger, M. (1967). J. Ultrastruct. Res. 17, 127.

Paweletz, N., and Granzow, C. (1972). Z. Zellforsch. 135, 71.

Pawlowski, P. J., and Berlowitz, L. (1969). Exp. Cell Res. 56, 154.

Perrin, A. (1969). C.R. Acad. Sci. Paris 269, 570.

Perrin, A. (1970). Protoplasma 70, 131.

Peterson, J. B., Gray, R. H., and Ris, H. (1972). J. Cell Biol. 53, 837.

Petrov, P., and Bernhard, W. (1971). J. Ultrastruct. Res. 35, 386.

Pfleger, R. C., Anderson, N. G., and Snyder, F. (1968). Biochemistry 7, 2826. Phillips, D. M. (1970). J. Cell Biol. 44, 243.

Picheral, B. (1970). J. Microsc. 9, 979.

Picheral, B. (1972a ). Z. Zellforsch. 131, 347.

Picheral, B. (1972b). Z. Zellforsch. 131, 371.

Picheral, B., and Folliot, R. (1971). J. Microsc. 12, 459.

Pickett-Heaps, J. (1969). Cytobios. 3, 257.

Pickett-Heaps, J. (1970). Protoplasma 70, 325.

Pickett-Heaps, J. (1971). Cytobios 3, 205.

Pickett-Heaps, J. (1972). Ann. Bot. 36, 693.

Pickett-Heaps, J., and Fowke, L. C. (1969). Aust. J. Biol. Sci. 22, 857.

Pickett-Heaps, J., and Northcote, D. H. (1966). J. Cell Sci. 1, 109.

Pitelka, D. R. (1969). In "Research in Protozoology" (T. T. Chen, ed.), p. 279. Pergamon, Oxford.

Pitot, H. C., Sladek, N., Ragland, W., Murray, R. K., Moyer, G., Soling, H. D., and Jost, J. P. (1969). In "Microsomes and Drug Oxidations" (J. R. Gillette, A. H. Conney, G. J. Cosmides, R. W. Estabrook, J. R. Fonts, and G. J. Mannering, eds.), p. 59. Academic Press, New York.

Plattner, H. (1971). J. Submicros. Cytol. 3, 19.

Plöen, L. (1971). Z. Zellforsch. 115, 553.

Pollister, A. W., Gettner, M., and Ward, R. (1954). Science 120, 789.

Porter, K. R., and Machado, R. D. (1960). J. Biophys. Biochem. Cytol. 7, 167.

Poux, N. (1969). J. Microsc. 8, 855.

Price, M. R., Harris, J. R., and Baldwin, R. W. (1972). J. Ultrastruct. Res. 40, 178. 
Przelecka, A. (1968). In "Cell Structure and its Interpretation" (S. M. McGee-Russel and K. F. A. Ross), pp. 157-167. Arnold, London.

Pusa, K. (1963). Proc. Int. Congr. Genet., 11th, The Hague, 1963.1, 110.

Quincey, R. V., and Wilson, S. H. (1969). Proc. Nat. Acad. Sci. U.S. 64, 981.

Rabl, C. (1885). Morphol. Jahrbuch 10, 214.

Radouco-Thomas, C., Nosal, G. L., and Radouco-Thomas, S. (1971). In "Chemistry and Brain Development" (R. Paoletti and A. N. Davison, eds.), pp. 291-308. Plenum Press, New York.

Rae, P. M. M., and Franke, W. W. (1972). Chromosoma 39, 443.

Raikov, I. M. (1969). In "Research in Protozoology" (T. T. Chen, ed.), Vol. 3, pp. 1-128. Pergamon, Oxford.

Raikov, I. M. (1973). C. R. Acad. Sci. Paris 276, 2385.

Rattner, J. B. (1972). J. Ultrastruct. Res. 40, 498.

Rattner, J. B., and Brinkley, B. R. (1971). J. Ultrastruct. Res. 36, 1.

Ray, T. K., Skipski, V. P., Barclay, M., Essner, E., and Archibald, F. M. (1969). J. Biol. Chem. 244, 5528.

Ray-Chaudhuri, S. P., Singh, L., and Sharma, T. (1971). Chromosoma 33, 239.

Rebhun, L. I. (1956). J. Biophys. Biochem. Cytol. 2, 93.

Rebhun, L. I. (1957). J. Biophys. Biochem. Cytol. 3, 509.

Rebhun, L. I. (1961). J. Ultrastruct. Res. 5, 208.

Reger, J. F. ( 1967 ). Z. Zellforsch. 82, 257.

Reger, J. F. (1969). J. Ultrastruct. Res. 28, 422.

Reger, J. F., and Florendo, N. T. (1969). J. Ultrastruct. Res. 28, 235.

Reverberi, G. (1972). Acta Embryol. Exp. 135.

Reverberi, G., and DeLeo, G. (1972). Acta Embryol. Exp. 65.

Rhodin, J. A. G. (1962). J. Ultrastruct. Res. 6, 171.

Riemann, W., Muir, C., and MacGregor, H. C. (1969). J. Cell Sci. 4, 299.

Rimpau, J., and Lelley, T. (1972). Z. Pflanzenzüchtg. 67, 197.

Ringborg, U., and Rydlander, L. (1971). J. Cell Biol. 51, 355.

Ringborg, U., Daneholt, B., Edström, J.-E., Egyházi, E., and Lambert, B. (1970). J. Mol. Biol. 51, 327.

Ritch, R., and Philpott, C. W. (1969). Exp. Cell Res. 55, 17.

Robbins, E., and Borun, T. W. (1967). Proc. Nat. Acad. Sci. U.S. 57, 409.

Robbins, E., Gonatas, N. K., and Micali, A. (1964). J. Cell Biol. 21, 429.

Robbins, E., Pederson, T., and Klein, P. (1970). J. Cell Biol. 44, 400.

Roberts, K., and Northcote, D. H. (1970). Nature (London) 228, 385.

Roberts, K., and Northeote, D. H. (1971). Micros. Acta 71, 102.

Robertson, J. D. (1959). In "The Structure and Function of Subcellular Components" (E. M. Crook, ed.), p. 3. Cambridge Univ. Press, London and New York.

Robertson, J. D. (1964). In "Cellular Membranes in Development" (M. Locke, ed.), pp. 1-82. Academic Press, New York.

Robinow, C. F., and Marak, J. (1966). J. Cell Biol. 29, 129.

Robison, W. G. (1966). J. Cell Biol. 29, 251.

Romen, W, and Bannasch, P (1973). Virchows Arch. Abt. B Zellpath. 13, 267.

Rose, H. G., and Frenster, J. H. (1965). Biochim. Biophys. Acta 106, 577.

Roth, L. E., Obetz, S. W., and Daniels, S. W. (1960). J. Biophys. Biochem. Cytol. 8, 207.

Roth, L. E., Pihlaja, D. J., and Shigenaka, Y. (1970). J. Ultrastruct. Res. 30, 7.

Roth, T. F., and Parchman, L. G. (1971). Chromosoma 35, 9.

Rowley, M. J., Berlin, J. D., and Heller, C. G. (1971). Z. Zellforsch. 112, 139. 
Ruby, J. R., and Webster, R. M. (1972). Z. Zellforsch. 133, 1.

Rupec, M. (1969). Naturwissenschaften 56, 223.

Ryser, H. J. P., Gabathuler, M. P., and Roberts, A. B. (1971). In "Biomembranes"

(L. A. Manson, ed.), Vol. 2, pp. 197-209. Plenum Press, New York.

Ryser, U. (1970). Z. Zellforsch. 110, 108.

Ryter, A. (1968). Bacteriol. Rev. 32, 39.

Sachs, M. I., and Anderson, E. (1970). J. Cell Biol. 47, 140.

Sakai, A. (1968). Cytologia 33, 318.

Sakai, A. (1969a). Cytologia 34, 57.

Sakai, A. (1969b). Cytologia 34, 593.

Samarina, O. P., Lukanidin, E. M., Molnar, J., and Georgiev, G. P. (1968). J. Mol. Biol. 33, 251.

Samorajski, T., Ordy, J. M., Zeman, W., and Curtis, H. J. (1968). J. cell Biol. $38,213$.

Sandoz, D. (1970). J. Micros. 9, 535.

Sato, T., Fujii, T., Matsuura, T., and Ueda, K. (1972). J. Biochem. 72, 1049.

Scalzi, H. A., and Bahr, G. F. (1968). J. Ultrastruct. Res. 24, 116.

Scharrer, B., and Wurzelmann, S. (1969a). Z. Zellforsch. 96, 325.

Scharrer, B., and Wurzelmann, S. (1969b). Z. Zellforsch. 101, 1.

Scheer, U. (1970a). Thesis, Univ. of Freiburg, pp. 1-174.

Scheer, U. (1970b). J. Cell Biol. 45, 445.

Scheer, U. (1972). Z. Zellforsch. 127, 127.

Scheer, U. (1973). Develop. Biol. 30, 13.

Scheer, U., and Franke, W. W. (1969). J. Cell Biol. 42, 519.

Scheer, U., and Franke, W. W. (1972). Planta 107, 145.

Scheer, U., and Franke, W. W. (1974). In preparation.

Scheer, U., Trendelenburg, M. F., and Franke, W. W. (1973). Exp. Cell Res. $80,175$.

Scherbaum, O. (1957). Exp. Cell Res. 13, 24.

Schjeide, O. A. (1970). In "Cell Differentiation" (O. A. Schjeide and J. deVellis, eds.), p. 169. Van Nostrand Reinhold, Princeton, New Jersey.

Schjeide, O. A., Galey, F., Grellert, E. A., San Lin, R. I., de Vellis, J., and Mead, J. F. (1970). Biol. Reprod. $2,14$.

Schjeide, O. A., Nicholls, T., and Graham, G. (1972). Z. Zellforsch. 129, 1.

Schmidt, W. J. (1929). Protoplasma 7, 353.

Schmidt, W. J. (1932). Arch. Prot. 77, 463.

Schmidt, W. J. (1937). "Die Doppelbrechung von Karyoplasma, Zytoplasma und Metaplasma," Protoplasma-Monographien 11, pp. 1-388. Borntraeger, Berlin.

Schneider, W. C., and Kuff, E. L. (1969). J. Biol. Chem. 244, 4843.

Schnepf, E. (1960). Ber Deutsch. Bot. Ges. 73, 243.

Schnepf, E., and Koch, W. (1966). Arch Mikrobiol. 54, 229.

Scholz, W., and Paweletz, N. (1969). Z. Krebsforsch. 72, 211.

Schulz, S. R., and Jensen, W. A: (1968). Amer. J. Bot. 55, 541.

Schwalm, F. E. (1969). Wilhelm Roux' Arch. 162, 41.

Scott, R. E., Carter, R. L., and Kidwell, W. R. (1971). Nature New Biol. (London) 233, 219.

Sebuwufu, P. H. (1966). Nature (London) 212, 1382.

Sedar, A. W. (1969). J. Cell Biol. 43, 179.

Seed, J. (1966). J. Cell Biol. 28, 263.

Shapot, V. S., and Davidova, S. Y. (1971). Progr. Nucleic Acid Res. Mol. Biol. II, 81 . 
Sichel, G. (1966). La Cellule 66, 97.

Siebert, G. (1972). Subcell. Biochem. 1, 277.

Siebert, G., and Langendorf, H. (1970). Naturwissenschaften 57, 119.

Sillevis Smitt, W. W., Vlak, J. M., Schiphof, R., and Rozijn, T. H. (1972). Exp. Cell Res. 71, 33.

Singh, L. ( 1972 ). Chromosoma 38, 185.

Sjöstrand, F. S. (1968). In "The Membranes" (A. J. Dalton and F. Haguneau, eds.), pp. 151-210. Academic Press, New York.

Slankis, T., and Gibbs, S. P. (1972). J. Phycol. 8, 243.

Small, J. V., and Davies, H. G. (1970). J. Cell Sci. 7, 15.

Smith, D. S. (1970). J. Cell Biol. 47, 195a.

Smith, G. F., and O'Hara, P. T. (1968). J. Ultrastruct. Res. 21, 415.

Smith, S. J., Adams, H. R., Smetana, K., and Busch, H. (1969). Exp. Cell Res. $55,185$.

Smyth, J. D., Morseth, D. J., and Smyth, M. M. (1969). The Nucleus 12, 47.

Solari, A. J. (1972). Chromosoma 39, 237.

Sorsa, V. (1972). Hereditas 72, 215.

Soyer, M.-O. (1969a). J. Microsc. 8, 569.

Soyer, M.-O. (1969b). J. Microsc. 8, 709.

Soyer, M.-O. (1969c). C. R. Acad. Sci. Paris 268, 2082.

Soyer, M.-O. (1971). Chromosoma 33, 70.

Speth, V., and Wunderlich, F. (1970). J. Cell Biol. 47, 772.

Spirin, A. S. (1969). Eur. J. Biochem. 10, 20.

Sprey, B., and Gietz, N. (1973). Z. Pflanzenphysiol. 68, 397.

Sprey, B., and Hasche, A. (1971). Ber. Deutsch. Bot. Ges. 84, 261.

Sprey, B., and Hasche, A. (1972). Cytologia 37, 721.

Stadler, J., and Franke, W. W. (1972). Nature New Biol. 237, 237.

Stadler, J., and Franke, W. W. (1973). Biochim. Biophys. Acta 311, 205.

Stadler, J., and Kleinig, H. (1971). Biochim. Biophys. Acta 233, 315.

Stadler, J., and Franke, W. W. (1974). J. Cell Biol. 60, 297.

Stang-Voss, C. (1970). Z. Zellforsch. 109, 287.

Stang-Voss, C., and Staubesand, J. (1970). Z. Zellforsch. 111, 127.

Stanley, H. P. (1969). J. Ultrastruct. Res. 27, 230.

Stanley, H. P. (1971a). J. Ultrastrust. Res. 36, 86.

Stanley, H. P. (1971b). J. Ultrastruct. Res. 36, 103.

Starke, F.-J. ( 1971). Z. Zellforsch. 119, 483.

Stefanini, M., Oura, C., and Zamboni, L. (1969). J. Submicr. Cytol. 1, 1.

Stelly, N., Stevens, B. J., and Andrè, J. (1970). J. Microsc. 9, 1015.

Stevens, A. R. (1967). In "The Control of Nuclear Activity" (L. Goldstein, ed.), pp. 189-271. Prentice Hall, Englewood Cliffs, New Jersey.

Stevens, B. J. (1965). J. Cell Biol. 24, 349.

Stevens, B. J., and Andrè, J. (1969). In "Handbook of Molecular Cytology"

(A. Lima-de-Faria, ed.), pp. 837-871. North-Holland Publ., Amsterdam.

Stevens, B. J., and Swift, H. (1966). J. Cell Biol. 31, 55.

Stevenson, I. (1972). Aust. J. Biol. Sci. 25, 775.

Stevenson, I., and Lloyd, F. P. (1971). Aust. J. Biol. Sci. 24, 977.

Stoermer, E. F., Pankratz, H. S., and Bowen, C. C. (1965). Amer. J. Bot. 52, 1067.

Stoltz, D. B., Pavan, C., and Da Cunha, A. B. (1973). J. gen. Virol. 19, 145.

Strasburger, E. (1888). Histol. Beiträge 1, 1.

Strum, J. M., and Karnovsky, M. J. (1970). J. Cell Biol. 44, 655. 
Strum, J. M., Wicken, J., Stanbury, J. R., and Karnovsky, M. J. (1971). J. Cell Biol. 51, 162.

Stubblefield, E., and Wray, W. (1971). Chromosoma 32, 262.

Suganuma, Y. (1969). J. Nara Med. Assoc. 20, 323.

Summers, M. D. (1969). J. Virol. 4, 188.

Summers, M. D. (1971). J. Ultrastruct. Res. 35, 606.

Sved, J. A. (1966). Genetics 53, 747.

Swale, E. M. F. (1969). Arch. Mikrobiol. 67, 71.

Swift, H. (1958). In "The Chemical Basis of Development" (W. D. McElroy and B. Glass, eds.) p. 174. Johns Hopkins Press, Baltimore, Maryland.

Switt, H. (1965). Chromosome Struct. Funct. Aspects Symp. 1, 26.

Sylvester, E. S., and Richardson, J. (1970). Virology 42, 1023.

Szollosi, D. (1965). J. Cell Biol. 25, 545.

Szollosi, D., Calarco, P. G., and Donahue, R. P. (1972a). Anat. Rec. 174, 325.

Szollosi, D., Calarco, P., and Donahue, R. P. (1972b). J. Cell Sci. 11, 521.

Takamoto, T. (1966). Nature (London) 211, 772.

Tamura, S., Tsuruhara, T., and Watanabe, Y. (1969). Exp. Cell Res. 55, 351.

Tan, E. M., and Lerner, R. A. (1972). J. Mol. Biol. 68, 107.

Tanaka, K. (1970). Protoplasma 70, 423.

Tashiro, Y., Matsuura, S., Morimoto, T., and Nagata, S. (1968). J. Cell Biol. 36, C5. Tata, J. R., Hamilton, M. J., and Cole, R. D. (1972). J. Mol. Biol. 67, 231.

Teigler, D. J., and Baerwald, R. J. (1972). Tissue \& Cell 4, 447.

Terzakis, J. A., (1965). J. Cell Biol. 27, 293.

Terzakis, J. A., Sprinz, H., and Ward, R. A. (1967). J. Cell Biol. 34, 311.

Thair, B. W., and Wardrop, A. B. (1971). Planta 100, 1.

Thoenes, W. (1964). J. Ultrastruct. Res. 10, 194.

Thomas, C. (1970). Biochem. Biophys. Acta 224, 99.

Thomas, R. J. (1964). J. Cell Biol. 23, 95a.

Tilney, L. G. (1971). In "Origin and Continuity of Cell Organelles" (I. Reinert and H. Ursprung, eds.), pp. 222-260. Springer Verlag, Berlin.

Tokuyasu, K., Madden, S. C., and Zeldis, L. J. (1968). J. Cell Biol. 39, 630.

Tremblay, G. Y., Daniels, M. J., and Schaechter, M. (1969). J. Mol. Biol. 40, 65.

Tucker, J. B. (1967). J. Cell Sci. 2, 481.

Ueda, K., Matsuura, T., Date, N,, and Kawai, K. (1969). Biochem. Biophys. Res. Commun. 34, 322.

Ulrich, E. (1969). J. Microsc. 8, 447.

Unger, E., Strunk, C., and May, R. (1971). Z. Allg. Mikrobiol. 11, 443.

Urban, P. (1969). J. Cell Biol. 42, 606.

Vanderlyn, L. (1948). Bot. Rev. 41, 270.

Vazquez-Nin, G., and Bernhard, W. (1971). J. Ultrastruct. Res. 36, 842.

Verhey, C. A., and Moyer, F. H. (1967). J. Exp. Zool. 164, 195.

Vivier, E. (1967). J. Micros. 6, 371.

Wartenberg, H. (1962). Z. Zellforsch. 58, 427.

Watson, M. L. (1954), Biochim. Biophys. Acta 15, 475.

Watson, M. L. (1955). J. Biophys. Biochem. Cytol. 1, 257.

Watson, M. L. (1959). J. Biophys. Biochem. Cytol. 6, 147.

Watson, M. L. (1962). J. Cell Biol. 13, 162.

Watson, M. W., and Arnott, H. J. (1973). J. Phycol. 9, 15.

Wecke, J., and Giesbrecht, P. (1970). Septieme Congr. Microsc. Electron., Grenoble 233. 
Weiss, E. (1965). Pathol. Vet. 2, 514.

Wergin, W. P., Gruber, P. J., and Newcomb, E. H. (1970). J. Ultrastruct. Res. $30,533$.

Werner, G. (1966). Z. Zellforsch. 73, 576.

Wells, K. (1970). Mycologia 62, 761.

Wells, K. (1972). Univ. Calif. Publ. Bot. 62, 1.

Werz, G. (1964). Planta 62, 255.

Weston, J. C., Ackerman, G. A., Greider, M. H., and Nikolewski, R. F. (1972). Z. Zellforsch. 123, 153.

Weston, J. C., Greider, M. H., Ackerman, G. A., and Nikolewski, R. F. (1965). J. Appl. Phys. 36, 2627.

von Wettstein, D. (1971). Proc. Nat. Acad. Sci. U.S. 68, 851.

Wettstein, R., and Sotelo, J. R. (1967). J. Microsc. 6, 557.

Whaley, W. G. (1966). In "Organisation der Zelle. III. Probleme der biologischen Redupliation" (P. Sitte, ed.), pp. 340-371. Springer Verlag, Berlin.

Whaley, W. G., Dauwalder, M., and Kephart, J. E. (1971). In "Origin and Continuity of Cell Organelles" (J. Reinert and H. Ursprung, eds.) pp. 1-45. Springer Verlag, Berlin.

Whittle, E. D., Bushnell, D. E., and Potter, V. R. (1968). Biochim. Biophys. Acta 161, 41.

Widnell, C. C., and Siekevitz, P. (1967). J. Cell Biol. 35, 142a.

Wiener, J., Spiro, D., and Loewenstein, W. R. (1965). J. Cell Biol. 27, 107.

Williams, C. A., and Ockey, C. H. (1970). Exp. Cell Res. 63, 365.

Williamson, R. (1970). J. Mol. Biol. 51, 157.

Wilson, H. J. (1969). J. Cell Biol. 40, 854.

Wilson, H. J. (1970). Planta 94, 184.

Wischnitzer, S. (1958). J. Ultrastruct. Res. 1, 201.

Wischnitzer, S. (1960). Int. Rev. Cytol. 10, 137.

Wischnitzer, S. (1970). Int. Rev. Cytol. 27, 65.

Wise, G. E., and Goldstein, L. (1972). Chromosoma 36, 176.

Wise, G. E., and Goldstein, L. (1973). J. Cell Biol. 56, 129.

Wise, G. E., Stevens, A. R., and Prescott, D. M. (1972). Exp. Cell Res. 75, 347.

Wolf, U., Flinspach, G., Böhm, R., and Ohno, S. (1965). Chromosoma 16, 609.

Wolfe, J. (1972). Advan. Cell Mol. Biol. 2, 151-192.

Wolstenholme, D. R. ( 1966). Chromosoma 19, 449.

Woodcock, C. L. F. (1971). J. Cell Sci. 8, 611.

Woodcock, C. L. F., and Fernández-Morán, H. (1968). J. Mol. Biol. $31,627$.

Woodcock, C. L. F., and Miller, G. J. (1973). Protoplasma (Vienna) 77, 331.

Wooding, F. B. P., and O'Donnell, J. M. (1971). J. Ultrastruct. Res. 35, 71.

Wood, R. L. (1966). J. Cell Biol. 31, 125a.

Woollam, D. H. M., Ford, E. H. R., and Millen, J. W. (1966). Exp. Cell Res. 42, 657.

Woollam, D. H. M., Millen, J. W., and Ford, E. H. R. (1967). Nature (London) $213,298$.

Wu, R. S., and Warner, J. R. (1971). J: Cell Biol. 51, 643.

Wunderlich, F. (1969a). Exp. Cell Res. 56, 369.

Wunderlich, F. (1969b). Z. Zellforsch. 101, 581.

Wunderlich, F. (1972) J. Membrane Biol. 7, 220.

Wunderlich, F., and Franke, W. W. (1968). J. Cell Biol. 38, 458.

Wunderlich, F., and Speth, V. (1970). Protoplasma 70, 139.

Wunderlich, F., and Speth, V. (1972). J. Microsc. 13, 361. 
Yamada, K. M., Spooner, B. S., and Wessells, N. K. (1971). J. Cell Biol. 49, 614.

Yamamoto, T. (1962). Int. Congr. Electron Microsc., 5th LL-6. Academic Press, New York.

Yamamoto, T. (1963): J. Cell Biol. 17, 413.

Yasuzumi, G. (1956). J. Biochem. Biophys. Cytol. 2, 445.

Yasuzumi, G., and Ishida, H. (1957). J. Biophys. Biochem. Cytol. 3, 663.

Yasuzumi, G., and Sugihara, R. (1965). Exp. Cell Res. 37, 207.

Yasuzumi, G., and Tsubo, J. (1966). Exp. Cell Res. 43, 281.

Yasuzumi, G., Nakai, Y., Tsubo, J., Yasuda, M., and Sugioka, T. (1967). Exp. Cell Res. 45, 261.

Yasuzumi, G., Tsubo, J., Okada, K., Terawaki, A., and Enomoto, Y. (1968). J. Ultrastruct. Res. 23, 321 .

Yasuzumi, G., Tsubo, I., Yasuda, M., Sugioka, T., Sakamoto, H., and Yasuzumi, F. (1969). Proc. Int. Symp., 1st, Rome-Siena, 1969. Accad. Naz. Lincei 137, 401.

Yasuzumi, G., Matano, Y., Asai, T., Nagasaka, M., and Yasuzumi, F. (1971). Z. Zellforsch. 115, 543.

Yoo, B. Y., and Bayley, S. T. (1967). J. Ultrastruct. Res. 18, 651.

Yoshida, S., Modak, M. J., and Yagi, K. (1971). Biochem. Biophys. Res. Commun. 43, 1408.

Yoshikawa-Fukada, M., and Ebert, J. D. (1971). Biochem. Biophys. Res. Commun. $43,133$.

Yunis, J. J., and Yasmineh, W. G. (1971). Science 174, 1200.

Zamboni, L. (1971). "Fine Morphology of Mammalian Fertilization.” Harper \& Row, New York.

Zbarsky, I. B. (1972a). Progr. Mod. Biol. Acad. Sci. USSR 73, I.

Zbarsky, I. B. (1972b). Methods Cell Physiol. 5, 167-198.

Zbarsky, I. B., Perevoshchikova, K. A., and Delektorskaya, L. N. (1967). Dokl. Akad. Nauk. USSR (Cytol.) 177, 445.

Zbarsky, I. B. Pokrovsky, A. A., Perevoshchikova, K. A., Gapparov, M. M., Lasheneva, N. V., and Delektorskaya, L. N. (1968). Dokl. Akad. Nauk. USSR (Biochem.) 181, 993.

Zbarsky, I. B., Perevoshchikova, K. A., Delektorskaya, L. N., Delektorskaya, L. N., and Delektorsky, V. V. (1969). Nature (London) 221, 257.

Zeigel, R. F., and Dalton, H. J. (1962). J. Cell Biol. 15, 45.

Zentgraf, H., Deumling, B., Jarasch, E. D., and Franke, W. W. (1971). J. Biol. Chem. 246, 2986.

Zentgraf, H., Boichut, E., and Franke, W. W. (1972). Cytobiologie 6, 51.

Zentgraf, H., and Franke, W. W. (1974). In preparation.

Zibrin, M. (1972). Z. Zellforsch. 135, 155.

Zickler, D. (1970). Chromosoma 30, 287.

Zickler, D. (1973). Histochemie 34, 227.

Zissler, D. (1969). Z. Zellforsch. 96, 106. 